\title{
Rh(III)-Catalyzed Imidoyl C-H Carbamylation and Cyclization to Bicyclic [1,3,5]Triazinones
}

\author{
Danielle N. Confair, ${ }^{\ddagger}$ Nathaniel S. Greenwood, ${ }^{\ddagger}$ Brandon Q. Mercado, and Jonathan A. Ellman* \\ Department of Chemistry, Yale University, New Haven, Connecticut 06520, United States \\ jonathan.ellman@yale.edu
}

\section{Supporting Information}

\section{Table of Contents}

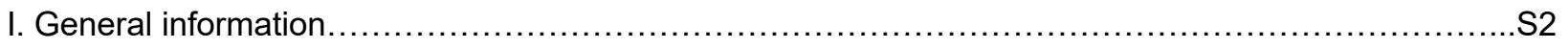

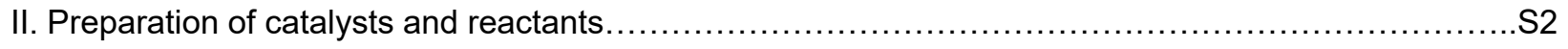

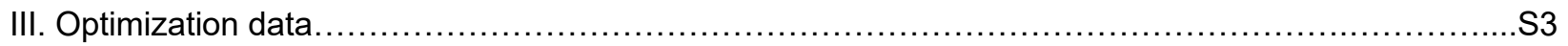

IV. Procedures and characterization data for synthesis of carbamylating reagents $2 \ldots \ldots \ldots \ldots \ldots \ldots \ldots . . . . \ldots$

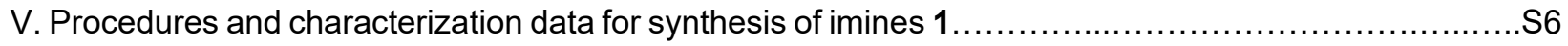

VI. Procedures and characterization data for two-component synthesis of triazinones $3 \ldots \ldots \ldots \ldots \ldots \ldots . . . . . \ldots 18$

VII. Procedures and characterization data for three-component synthesis of triazinones $3 \ldots \ldots \ldots \ldots \ldots \ldots . . .529$

VIII. Procedure and characterization data for $1.0 \mathrm{mmol}$ scale three-component reaction...................S33

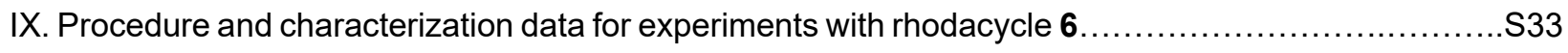

X. Procedure and characterization data for experiments with uncyclized intermediate $7 \ldots \ldots \ldots \ldots \ldots \ldots . . . . \ldots 34$

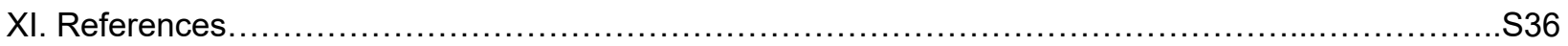

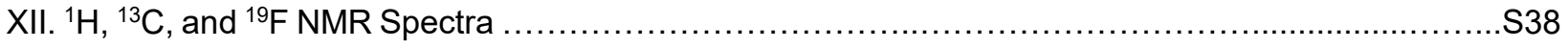

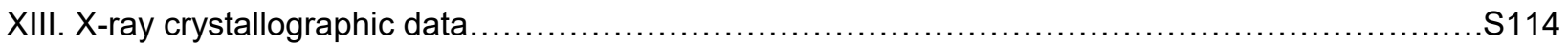




\section{General information}

Solvents including toluene and 1,4-dioxane were purified by elution through a column of activated alumina under an argon atmosphere. Hexafluoroisopropanol (HFIP), 1,2-dichloroethane (DCE), 2,2,2trifluoroethanol (TFE), and methanol $(\mathrm{MeOH})$ were deoxygenated by sparging with argon. All reaction solvents were stored over $3 \AA$ molecular sieves in a glovebox. For work-up and purification, ACS reagent grade solvents were used. All other reagents were purchased from commercial sources and used without further purification.

Flash-column chromatography was performed on SiliaFlash® P60 silica gel (230-400 mesh), and silica gelcoated glass plates from Analtech $(1 \mathrm{~mm} \mathrm{SiO}, 20 \times 20 \mathrm{~cm})$ were used for preparative thin-layer chromatography.

For NMR characterization, the magnetic field strength of the instrument is indicated for each spectrum. NMR chemical shifts $(\delta)$ are reported in ppm relative to $\mathrm{CHCl}_{3}(\delta=7.26 \mathrm{ppm}), \mathrm{C}_{6} \mathrm{H}_{6}(\delta=7.16 \mathrm{ppm}), \mathrm{MeOH}$ $(\delta=3.31 \mathrm{ppm})$, or $\left(\mathrm{CH}_{3}\right)_{2} \mathrm{SO}(\delta=2.50 \mathrm{ppm})$ for ${ }^{1} \mathrm{H} \mathrm{NMR}$, and $\mathrm{CDCl}_{3}(\delta=77.16 \mathrm{ppm}), \mathrm{C}_{6} \mathrm{D}_{6}(\delta=128.06$ $\mathrm{ppm}), \mathrm{CD}_{3} \mathrm{OD}(\delta=49.00 \mathrm{ppm})$, or $\left(\mathrm{CD}_{3}\right)_{2} \mathrm{SO}(\delta=39.52 \mathrm{ppm})$ for ${ }^{13} \mathrm{C}-\mathrm{NMR}$. All ${ }^{13} \mathrm{C}$ NMR are proton decoupled. The multiplicity and shape of NMR signals are designated by the following abbreviations: $s=$ singlet, $d=$ doublet, $t=$ triplet, $q=$ quartet, $p=$ pentet, $m=$ multiplet, and $b r=$ broad signal. Coupling constants $\mathrm{J}$ are reported in Hertz $(\mathrm{Hz})$. Partial data are provided for IR spectra. Melting points are reported uncorrected. Mass spectrometric measurements for imines 1c-1k, 1m-1n, 1p-1w, 1y, 1aa, and intermediate 7 were performed with a Thermo Fisher QExactive Orbitrap LC-MS system, equipped with a Dionex UltraMate 3000 UHPLC, consisting of a LPG-3400SD pump unit, a WPS-3000 autosampler and a Column Oven TCC-3000. UV data was collected with a Photodiode Array Detector DAD-3000 for 220, 280, 340 and 400nm. Mass spectra (MS and MS/MS) were subsequently recorded with the QExactive Orbitrap mass spectrometer. Each compound was directly injected into the flow path of the LC system without column separation. Electrospray was used for desolvatization and ionization ionization, with the electrospray needle held at $+3.5 \mathrm{kV}$. Mass spectrometric measurements for compounds 2, 2b, 2d, 3a-3k, 3n-3w, 3y-3ad, and 6 were performed with a Shimadzu Scientific Instruments QToF 9030 LC-MS system, equipped with a Nexera LC-40D xs UHPLC, consisting of a CBM-40 Lite system controller, a DGU-405 Degasser Unit, two LC-40D XS UHPLC pumps, a SIL-40C XS autosampler and a Column Oven CTO-40S. UV data was collected with a Shimadzu Nexera HPLC/UHPLC Photodiode Array Detector SPD M-40 in the range of $190-800 \mathrm{~nm}$. Mass spectra (MS and MS/MS) were subsequently recorded with the quadrupole time-of-flight (QToF) 9030 mass spectrometer. Samples were injected into a sample loop (max. $40 \mu \mathrm{L}$ ) and separated on a Shim-pack Scepter $\mathrm{C} 18-120,1.9 \mu \mathrm{m}, 2.1 \times 50 \mathrm{~mm}$ Column, equilibrated at $40^{\circ} \mathrm{C}$ in a column oven. The ionization source was run in "DUIS" mode, with the electrospray needle held at $+4.5 \mathrm{kV}$ and the corona discharge at $+1 \mathrm{kV}$.

\section{Preparation of catalysts and reactants}

Compounds $\mathbf{2 a},{ }^{1} \mathbf{2} \mathbf{e},{ }^{2}$ and $\mathbf{2} \mathbf{f}^{3}$ were prepared according to literature. Alkyl $N$-hydroxycarbamates were prepared according to literature ${ }^{4}$ and used without purification. All aldehydes and amines were purchased and used as received. $\mathrm{N}$-(3-Methylpyridin-2-yl)-1-phenylmethanimine (1s) was prepared as reported in the literature. ${ }^{5}\left[\mathrm{Cp}^{*} \mathrm{Rh}\left(\mathrm{MeCN}_{3}\right]\left(\mathrm{SbF}_{6}\right)_{2}\right.$ was synthesized according to literature procedures. ${ }^{6}$ 


\section{Optimization data}

\section{Evaluation of carbamylating reagents}<smiles>CCOC(=O)NOC(=O)C(C)(C)C</smiles>

2<smiles>CC(C)(C)OC(=O)NOC(=O)C(C)(C)C</smiles>

$2 \mathbf{a}$<smiles>COC(=O)NOC(=O)C(C)(C)C</smiles>

2b<smiles>CC(C)(C)C(=O)ONC(=O)OCC(F)(F)F</smiles>

2c<smiles>CC(C)(C)C(=O)ONC(=O)Oc1ccccc1</smiles>

2d<smiles>CCOC(N)=O</smiles>

$2 e$<smiles>CCOC(=O)NCl</smiles>

2f

Table S1. Reaction of imine 1a with carbamylating reagents $\mathbf{2 - 2 f ^ { a }}$

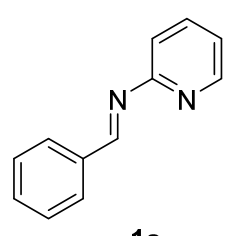

$1 \mathrm{a}$

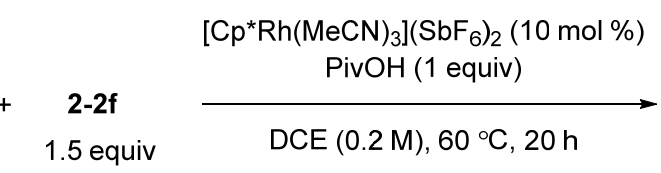

$\operatorname{DCE}(0.2 \mathrm{M}), 60^{\circ} \mathrm{C}, 20 \mathrm{~h}$

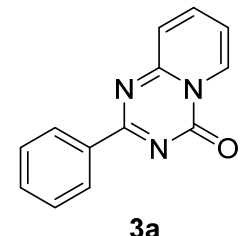

\begin{tabular}{llll}
\hline Entry & Carbamylating reagent & Variation from standard conditions & Yield $\mathbf{3 a}^{\mathrm{b}}(\%)$ \\
\hline 1 & $\mathbf{2}$ & none & 76 \\
2 & $\mathbf{2 a}$ & none & 72 \\
3 & $\mathbf{2 b}$ & none & 55 \\
4 & $\mathbf{2 c}$ & none & 76 \\
5 & $\mathbf{2 d}$ & none & 72 \\
6 & $\mathbf{2 e}$ & None & $0 \%$ \\
7 & $\mathbf{2 e}$ & $\mathrm{Cp}{ }^{*} \mathrm{Co}(\mathrm{CO}) I_{2}(10 \mathrm{~mol} \%)$ with $\mathrm{AgSbF}_{6}(20 \mathrm{~mol} \%)$ & $0 \%$ \\
& & instead of $\left[\mathrm{Cp}{ }^{*} \mathrm{Rh}(\mathrm{MeCN})_{3}\right]\left(\mathrm{SbF}_{6}\right)_{2}$ & \\
8 & $\mathbf{2 f}$ & none & $8 \%$ \\
9 & $\mathbf{2 f}$ & Cp ${ }^{*} \mathrm{Co}(\mathrm{CO}) I_{2}(10 \mathrm{~mol} \%)$ with $\mathrm{AgSbF}_{6}(20 \mathrm{~mol} \%)$ & $0 \%$ \\
& & instead of $\left[\mathrm{Cp}{ }^{*} \mathrm{Rh}(\mathrm{MeCN})_{3}\right]\left(\mathrm{SbF}_{6}\right)_{2}$ & \\
\hline
\end{tabular}

a. Conditions: $\mathbf{1 a}(0.1 \mathrm{mmol}), \mathbf{2 - 2 f}(0.15 \mathrm{mmol})$. b. Yield determined by ${ }^{1} \mathrm{H}$ NMR relative to trimethyl(phenyl)silane as an external standard. 
Table S2. Reaction of challenging o-substituted imine $1 \mathbf{g}$ with carbamylating reagents $2-2 \mathrm{a}, \mathbf{2 c}-\mathbf{2} \mathbf{d}^{\mathrm{a}}$

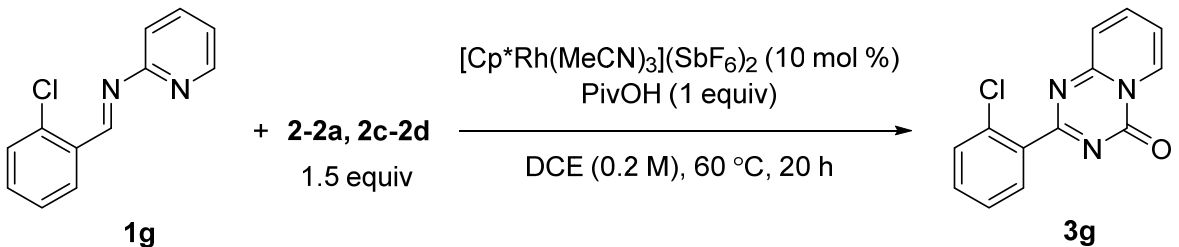

$1 \mathrm{~g}$

$3 \mathbf{g}$

\begin{tabular}{llll}
\hline Entry & Carbamylating reagent & Variation from standard conditions & Yield $\mathbf{3 g}^{\text {b }}(\%)$ \\
\hline 1 & $\mathbf{2}$ & none & 68 \\
2 & $\mathbf{2 a}$ & none & 2 \\
3 & $\mathbf{2 c}$ & none & 61 \\
4 & $\mathbf{2 d}$ & None & 70 \\
\hline
\end{tabular}

a. Conditions: $\mathbf{1 g}(0.1 \mathrm{mmol}), \mathbf{2 - 2 a}, \mathbf{2 c - 2 d}(0.15 \mathrm{mmol})$. b. Yield determined by ${ }^{1} \mathrm{H}$ NMR relative to trimethyl(phenyl)silane as an external standard.

\section{Evaluation of catalysts}

Table S3. Reaction of imine 1a with carbamylating reagent 2 using other catalysts ${ }^{a}$

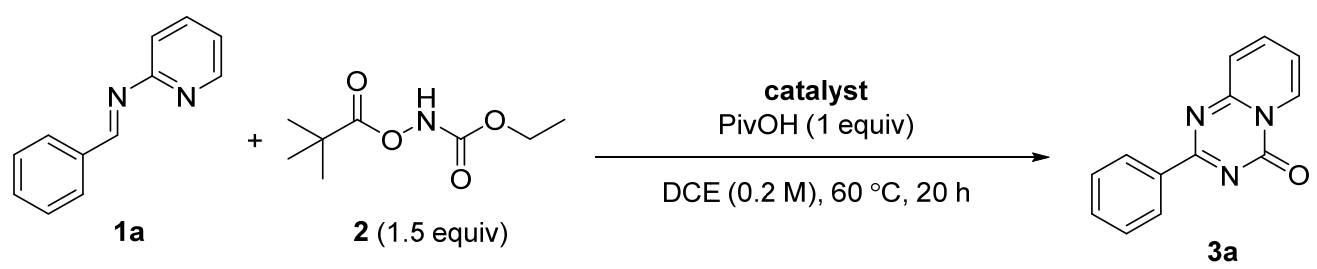

\begin{tabular}{lll}
\hline Entry & catalyst & \multicolumn{1}{c}{ Yield 3a $^{\mathrm{b}}(\%)$} \\
\hline 1 & $\left.\mathrm{Cp}^{*} \mathrm{Co}(\mathrm{CO})\right|_{2}(10 \mathrm{~mol} \%)+\mathrm{AgSbF}_{6}(20 \mathrm{~mol} \%)$ & 0 \\
2 & {$\left[\mathrm{Cp}^{*} \mid r \mathrm{Cl}_{2}\right]_{2}(5 \mathrm{~mol} \%)+\mathrm{AgSbF}_{6}(20 \mathrm{~mol} \%)$} & $11 \%$ \\
3 & {$\left[\mathrm{Cp}^{*} \mathrm{RhCl}_{2}\right]_{2}(5 \mathrm{~mol} \%)+\mathrm{AgSbF}_{6}(20 \mathrm{~mol} \%)$} & $57 \%$ \\
\hline
\end{tabular}

a. Conditions: $1 \mathrm{a}(0.1 \mathrm{mmol}), \mathbf{2}(0.15 \mathrm{mmol})$. b. Yield determined by ${ }^{1} \mathrm{H}$ NMR relative to trimethyl(phenyl)silane as an external standard.

\section{Optimization of three-component conditions}

Table S4. 3-Component reaction with benzaldehyde and 2-aminopyridine ${ }^{a}$

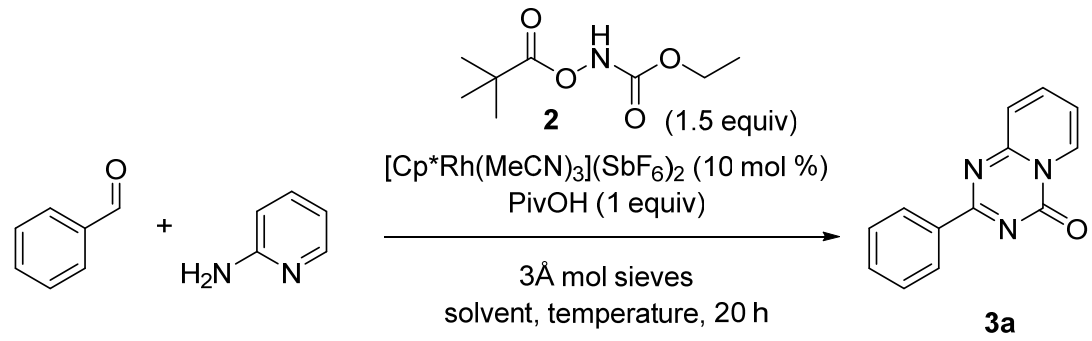




\begin{tabular}{lllll}
\hline Entry & Solvent & Equiv aldehyde & Temperature & Yield 3a $\mathbf{a}^{\mathrm{b}}$ (\%) \\
\hline 1 & TFE & 1.0 & $60^{\circ} \mathrm{C}$ & $38 \%$ \\
$2^{\mathrm{c}}$ & TFE & 1.0 & $60{ }^{\circ} \mathrm{C}$ & $17 \%$ \\
3 & TFE & 1.0 & $80^{\circ} \mathrm{C}$ & $39 \%$ \\
4 & TFE & 2.0 & $60^{\circ} \mathrm{C}$ & $67 \%$ \\
5 & TFE & 2.0 & $80^{\circ} \mathrm{C}$ & $63 \%$ \\
$6^{\mathrm{d}}$ & TFE & 2.0 & $80^{\circ} \mathrm{C}$ & $55 \%$ \\
$7^{\mathrm{e}}$ & TFE & 2.0 & $80^{\circ} \mathrm{C}$ & $45 \%$ \\
8 & DCE & 2.0 & $60^{\circ} \mathrm{C}$ & $33 \%$ \\
9 & DCE & 2.0 & $80^{\circ} \mathrm{C}$ & $51 \%$ \\
10 & HFIP & 2.0 & $80^{\circ} \mathrm{C}$ & $72 \%$ \\
\hline
\end{tabular}

a. Conditions: benzaldehyde $(0.20 \mathrm{mmol}), 2$-aminopyridine $(0.10 \mathrm{mmol}), 2(0.15 \mathrm{mmol})$. b. Yield determined by ${ }^{1} \mathrm{H}$ NMR relative to trimethyl(phenyl)silane as an external standard. c. without sieves. d. 0.4 M. e. with 1.0 equiv $\mathrm{NaOAc}$

\section{Procedures and characterization data for synthesis of carbamylating reagents 2}

\section{General procedure for the synthesis of carbamylating reagents:}

Procedure adapted from literature. ${ }^{2}$ In a round bottom flask with a magnetic stir bar was prepared a solution of crude alkyl $\mathrm{N}$-hydroxycarbamate (1 equiv) in anhydrous diethyl ether $(0.5 \mathrm{M})$. The solution was cooled to $0{ }^{\circ} \mathrm{C}$ and triethylamine ( 1 equiv) was added. Pivaloyl chloride (1 equiv) was added dropwise, and was stirred at $0{ }^{\circ} \mathrm{C}$ for $2 \mathrm{~h}$ and warmed to room temperature overnight. The crude mixture was filtered over a pad of celite, concentrated in vacuo, and purified by flash-column chromatography over silica gel.

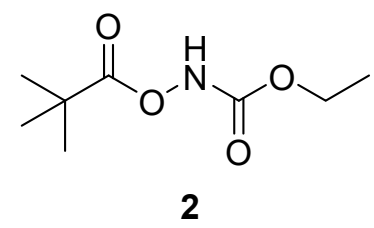

Ethyl (pivaloyloxy)carbamate (2): The reaction was set up according to the General Procedure using ethyl $\mathrm{N}$-hydroxycarbamate $(6.31 \mathrm{~g}, 60.0 \mathrm{mmol}, 1.0$ equiv), diethyl ether $(120 \mathrm{~mL}, 0.5 \mathrm{M})$, triethylamine $(8.36$ $\mathrm{mL}, 60.0 \mathrm{mmol}, 1$ equiv), and pivaloyl chloride $(7.38 \mathrm{~mL}, 60.0 \mathrm{mmol}, 1.0$ equiv). Purification by flash-column chromatography over silica gel (10\% EtOAc/hexanes) gave the desired product 2 as a colorless oil (9.82 g, $86 \%$ yield). IR (neat) 3278, 2979, 1777, 1738, 1480, 1244, 1097, 1011, 757, $561 \mathrm{~cm}^{-1} .{ }^{1} \mathrm{H}$ NMR $(400$ $\left.\mathrm{MHz} \mathrm{CDCl}_{3}\right) \delta 8.24(\mathrm{~s}, 1 \mathrm{H}), 4.17(\mathrm{q}, J=7.1 \mathrm{~Hz}, 2 \mathrm{H}), 1.24(\mathrm{~s}, 9 \mathrm{H}), 1.23(\mathrm{t}, J=7.1 \mathrm{~Hz}, 3 \mathrm{H}) .{ }^{13} \mathrm{C} \mathrm{NMR}(151$ $\left.\mathrm{MHz}, \mathrm{CDCl}_{3}\right) \delta 177.6,156.9,62.7,38.2,26.9,14.2$. HRMS $(\mathrm{ESI}+, \mathrm{m} / \mathrm{z}):[\mathrm{M}+\mathrm{H}]+$ calcd for $\mathrm{C}_{8} \mathrm{H}_{16} \mathrm{NO}_{4}{ }^{+}$: 190.1074, found: 190.1085 .<smiles>COC(=O)NOC(=O)C(C)(C)C</smiles> 
Methyl (pivaloyloxy)carbamate (2b): The reaction was set up according to the General Procedure using methyl $\mathrm{N}$-hydroxycarbamate $(1.95 \mathrm{~g}, 21.0 \mathrm{mmol}, 1.0$ equiv), diethyl ether $(42 \mathrm{~mL}, 0.5 \mathrm{M})$, triethylamine $(2.98$ $\mathrm{mL}, 21.0 \mathrm{mmol}, 1.0$ equiv), and pivaloyl chloride $(2.63 \mathrm{~mL}, 21.0 \mathrm{mmol}, 1.0$ equiv). Purification by flashcolumn chromatography over silica gel (20\% EtOAc/hexanes) gave the desired product $\mathbf{2} \mathbf{b}$ as a colorless oil $\left(3.29 \mathrm{~g}, 88 \%\right.$ ). IR (neat) 3278, 2977, 1778, 1742, 1480, 1459, 1250, 1106, 1081, 877, $756 \mathrm{~cm}^{-1}$. ${ }^{1} \mathrm{H}$ NMR $\left(500 \mathrm{MHz}, \mathrm{CDCl}_{3}\right) \delta 7.99$ (s, 1H), $3.80(\mathrm{~s}, 3 \mathrm{H}), 1.30$ (s, 9H). ${ }^{13} \mathrm{C} \mathrm{NMR}\left(151 \mathrm{MHz}, \mathrm{CDCl}_{3}\right) \delta 177.5,157.3$, 53.3, 38.1, 26.8. HRMS (ESI+, m/z): $[\mathrm{M}+\mathrm{H}]+$ calcd for $\mathrm{C}_{7} \mathrm{H}_{14} \mathrm{NO}_{4}{ }^{+}: 176.0917$, found: 176.0933 .

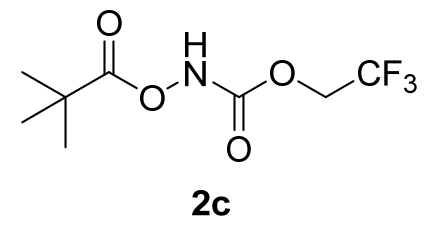

2,2,2-Trifluoroethyl (pivaloyloxy)carbamate (2c): The reaction was set up according to the General Procedure using 2,2,2-trifluoroethyl N-hydroxycarbamate $(1.59 \mathrm{~g}, 10.0 \mathrm{mmol}, 1.0$ equiv), diethyl ether (20 $\mathrm{mL}, 0.5 \mathrm{M})$, triethylamine $(1.39 \mathrm{~mL}, 10.0 \mathrm{mmol}, 1.0$ equiv), and pivaloyl chloride $(1.23 \mathrm{~mL}, 10.0 \mathrm{mmol}, 1.0$ equiv). Purification by flash-column chromatography over silica gel (20\% EtOAc/hexanes) gave the desired product 2c as a colorless oil (1.33 g, 54\% yield). IR (neat) 3232, 1781, 1744, 1482, 1292, 1167, 1113, 1082, 958, 837, $736 \mathrm{~cm}^{-1} .{ }^{1} \mathrm{H}$ NMR $\left(500 \mathrm{MHz}, \mathrm{CDCl}_{3}\right) \delta 8.09(\mathrm{~s}, 1 \mathrm{H}), 4.54(\mathrm{q}, J=8.2 \mathrm{~Hz}, 2 \mathrm{H}), 1.31(\mathrm{~s}, 9 \mathrm{H}) .{ }^{13} \mathrm{C}$ $\operatorname{NMR}\left(151 \mathrm{MHz}, \mathrm{CDCl}_{3}\right) \delta 177.4,154.9,122.7$ (q, $\left.J=277.2 \mathrm{~Hz}\right), 61.9(\mathrm{q}, J=37.1 \mathrm{~Hz}), 38.4,26.90 .{ }^{19} \mathrm{~F} \mathrm{NMR}$ $\left(376 \mathrm{MHz}, \mathrm{CDCl}_{3}\right) \delta-74.2$. The instability of this reagent prevented us from obtaining HRMS data.

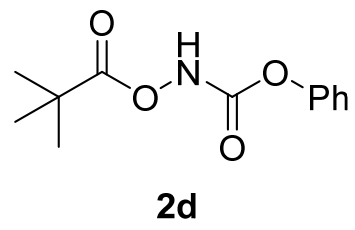

Phenyl (pivaloyloxy)carbamate (2d): The reaction was set up according to the General Procedure using phenyl $\mathrm{N}$-hydroxycarbamate $(1.53 \mathrm{~g}, 10.0 \mathrm{mmol}, 1.0$ equiv), diethyl ether $(20 \mathrm{~mL}, 0.5 \mathrm{M})$, triethylamine $(1.39$ $\mathrm{mL}, 10.0 \mathrm{mmol}, 1.0$ equiv), and pivaloyl chloride $(1.23 \mathrm{~mL}, 10.0 \mathrm{mmol}, 1.0$ equiv). Purification by flashcolumn chromatography over silica gel (15\% EtOAc/hexanes) gave the desired product $\mathbf{2} \mathbf{d}$ as a colorless oil $\left(2.18 \mathrm{~g}, 92 \%\right.$ yield). IR (neat) 3229, 1774, 1749, 1455, 1232, 1193, 1102, 1014, 794, $740 \mathrm{~cm}^{-1}$. ${ }^{1} \mathrm{H}$ NMR $\left(600 \mathrm{MHz}, \mathrm{CDCl}_{3}\right) \delta 8.61(\mathrm{~s}, 1 \mathrm{H}), 7.36(\mathrm{t}, J=8.4 \mathrm{~Hz}, 2 \mathrm{H}), 7.23(\mathrm{t}, J=7.2 \mathrm{~Hz}, 1 \mathrm{H}), 7.16(\mathrm{~d}, J=8.4 \mathrm{~Hz}, 2 \mathrm{H})$, 1.33 (s, 9H). ${ }^{13} \mathrm{C} \mathrm{NMR}\left(151 \mathrm{MHz}, \mathrm{CDCl}_{3}\right) \delta 177.6,155.0,150.2,129.5,126.1,121.3,38.3,26.9$. HRMS $(E S I+, m / z):[M+H]+$ calcd for $\mathrm{C}_{12} \mathrm{H}_{16} \mathrm{NO}_{4}{ }^{+}: 238.1074$, found: 238.1091 .

\section{Procedures and characterization data for synthesis of imines 1}

\section{General procedure for the synthesis of imines}

To a flame-dried $25 \mathrm{~mL}$ round-bottom flask was added amine (1.0 equiv), $3 \AA \AA$ molecular sieves $(0.5 \mathrm{~g} / \mathrm{mmol})$, dry toluene $(0.5 \mathrm{M})$, and aldehyde (1.0 equiv). The reaction solution was stirred under $\mathrm{N}_{2}$ at $80^{\circ} \mathrm{C}$ overnight. 
The crude reaction mixture was pipetted away from the molecular sieves, concentrated in vacuo, and purified by silica gel column chromatography.<smiles>C(=N/c1ccccn1)\c1ccccc1</smiles>

1a

$(E)-1-P h e n y l-N$-(pyridin-2-yl)methanimine (1a): ${ }^{7}$ The reaction was set up according to the General Procedure using 2-aminopyridine $(1.41 \mathrm{~g}, 15.0 \mathrm{mmol}, 1.0$ equiv), $3 \AA$ molecular sieves $(7.5 \mathrm{~g})$, toluene (30 $\mathrm{mL})$, and benzaldehyde ( $1.53 \mathrm{~mL}, 15.0 \mathrm{mmol}, 1.0$ equiv). Purification by flash-column chromatography over silica gel (15\% EtOAc/2\% Et 3 N/hexanes) gave the desired product $1 \mathrm{a}$ as a white solid $(1.34 \mathrm{~g}, 49 \%$ yield). Reported spectra match literature data. ${ }^{1}{ }^{1} \mathrm{H}$ NMR $\left(400 \mathrm{MHz}, \mathrm{CDCl}_{3}\right) \delta 9.15(\mathrm{~s}, 1 \mathrm{H}), 8.50$ (dd, $J=5.1,1.9$ $\mathrm{Hz}, 1 \mathrm{H}), 8.00(\mathrm{dd}, J=7.5,2.0 \mathrm{~Hz}, 2 \mathrm{H}), 7.76(\mathrm{td}, J=7.7,1.9 \mathrm{~Hz}, 1 \mathrm{H}), 7.55-7.44(\mathrm{~m}, 3 \mathrm{H}), 7.34(\mathrm{~d}, J=8.0$ $\mathrm{Hz}, 1 \mathrm{H}), 7.19$ (td, $J=7.4,4.9 \mathrm{~Hz}, 1 \mathrm{H}) .{ }^{13} \mathrm{C}$ NMR $\left(151 \mathrm{MHz}, \mathrm{CDCl}_{3}\right) \delta$ 163.0, 161.3, 149.0, 138.2, 136.0, 132.0, 129.6, 128.9, 122.0, 119.9 .<smiles>COc1ccc(/C=N/c2ccccn2)cc1</smiles>

$1 b$

(E)-1-(4-Methoxyphenyl)-N-(pyridin-2-yl)methanimine (1b): ${ }^{7}$ The reaction was set up according to the General Procedure using 2-aminopyridine (188 mg, $2.00 \mathrm{mmol}, 1.0$ equiv), $3 \AA$ molecular sieves ( $1.0 \mathrm{~g}$ ), toluene $(4 \mathrm{~mL})$, and 4-methoxybenzaldehyde $(0.24 \mathrm{~mL}, 2.0 \mathrm{mmol}, 1.0$ equiv). Purification by flash-column chromatography over silica gel (40\% EtOAc/2\% Et 3 N/hexanes) gave the desired product 1a as a white solid (327 mg, 77\% yield). Reported spectra match literature data ${ }^{7} .{ }^{1} \mathrm{H}$ NMR $\left(500 \mathrm{MHz}\right.$, Benzene- $\left.d_{6}\right) \delta 9.50$ (s, $1 \mathrm{H}), 8.41(\mathrm{~d}, J=4.8 \mathrm{~Hz}, 1 \mathrm{H}), 7.92(\mathrm{~d}, J=8.7 \mathrm{~Hz}, 2 \mathrm{H}), 7.36(\mathrm{~d}, J=7.9 \mathrm{~Hz}, 1 \mathrm{H}), 7.18(\mathrm{dd}, J=7.8,2.0 \mathrm{~Hz}$, $1 \mathrm{H}), 6.69(\mathrm{~d}, J=8.4 \mathrm{~Hz}, 2 \mathrm{H}), 6.65(\mathrm{t}, J=6.1 \mathrm{~Hz}, 1 \mathrm{H}), 3.18(\mathrm{~s}, 3 \mathrm{H}) \cdot{ }^{13} \mathrm{C}$ NMR $\left(126 \mathrm{MHz}\right.$, Benzene- $\left.d_{6}\right) \delta$ $163.0,162.1,161.8,149.2,137.8,131.7,129.9,121.5,120.8,114.5,54.8$.<smiles>C(=N/c1ccccn1)\c1ccccc1</smiles>

$1 \mathrm{c}$ 
(E)-1-([1,1'-Biphenyl]-4-yl)-N-(pyridin-2-yl)methanimine (1c): The reaction was set up according to the General Procedure using 2-aminopyridine (188 mg, $2.00 \mathrm{mmol}, 1.0$ equiv), $3 \AA$ molecular sieves $(1.0 \mathrm{~g})$, toluene $(4 \mathrm{~mL})$, and 4-phenylbenzaldehyde (364 mg, $2.0 \mathrm{mmol}, 1.0$ equiv). Purification by flash-column chromatography over silica gel ( $40 \% \mathrm{EtOAc} / 2 \% \mathrm{Et}_{3} \mathrm{~N} /$ hexanes) gave the desired product $1 \mathrm{c}$ as a white solid (353 mg, 68\% yield). mp 122-123 ${ }^{\circ} \mathrm{C}$. IR (neat) 1582, 1556, 1456, 1429, 1359, 1205, 1144, 981, 897, 844, $789,726 \mathrm{~cm}^{-1} .{ }^{1} \mathrm{H}$ NMR $\left(500 \mathrm{MHz}, \mathrm{CDCl}_{3}\right) \delta 9.21(\mathrm{~s}, 1 \mathrm{H}), 8.52(\mathrm{dd}, J=4.8,2.0 \mathrm{~Hz}, 1 \mathrm{H}), 8.07(\mathrm{~d}, J=8.3 \mathrm{~Hz}$, $2 \mathrm{H}), 7.77(\mathrm{td}, J=7.7,1.9 \mathrm{~Hz}, 1 \mathrm{H}), 7.73(\mathrm{~d}, J=8.5 \mathrm{~Hz}, 2 \mathrm{H}), 7.66(\mathrm{~d}, J=8.0 \mathrm{~Hz}, 2 \mathrm{H}), 7.48(\mathrm{t}, J=7.7 \mathrm{~Hz}, 2 \mathrm{H})$, $7.42-7.34(\mathrm{~m}, 2 \mathrm{H}), 7.20$ (ddd, $J=7.4,4.8,1.1 \mathrm{~Hz}, 1 \mathrm{H}) .{ }^{13} \mathrm{C} \mathrm{NMR}\left(151 \mathrm{MHz}, \mathrm{CDCl}_{3}\right) \delta 162.6,161.3,149.0$, $144.8,140.4,138.3,135.0,130.1,129.1,128.1,127.6,127.4,122.0,120.1$. HRMS (ESI+, m/z): [M+H] + calcd for $\mathrm{C}_{18} \mathrm{H}_{15} \mathrm{~N}_{2}{ }^{+}: 259.1230$, found: 259.1230 .<smiles>FC(F)(F)c1ccc(/C=N/c2ccccn2)cc1</smiles>

1d

(E)-N-(Pyridin-2-yl)-1-(4-(trifluoromethyl)phenyl)methanimine (1d): The reaction was set up according to the General Procedure using 2-aminopyridine ( $188 \mathrm{mg}, 2.00 \mathrm{mmol}, 1.0$ equiv), $3 \AA$ molecular sieves (1.0 $\mathrm{g}$ ), toluene $(4 \mathrm{~mL})$, and 4-(trifluoromethyl)benzaldehyde $(0.27 \mathrm{~mL}, 2.0 \mathrm{mmol}, 1.0$ equiv). Purification by flash-column chromatography over silica gel (30\% EtOAc/2\% $\mathrm{Et}_{3} \mathrm{~N} /$ hexanes) gave the desired product 1d as a white solid (432 mg, $86 \%$ yield). $\mathrm{mp} 89-91^{\circ} \mathrm{C}$. IR (neat) 1618, 1588, 1561, 1434, 1319, 1150, 1103 , 1062, 836, 742, $594 \mathrm{~cm}^{-1} .{ }^{1} \mathrm{H}$ NMR $\left(500 \mathrm{MHz}, \mathrm{CDCl}_{3}\right) \delta 9.23(\mathrm{~s}, 1 \mathrm{H}), 8.52(\mathrm{dd}, J=4.9,1.9 \mathrm{~Hz}, 1 \mathrm{H}), 8.11(\mathrm{~d}$, $J=8.0 \mathrm{~Hz}, 2 \mathrm{H}), 7.79(\mathrm{td}, J=7.7,1.9 \mathrm{~Hz}, 1 \mathrm{H}), 7.74(\mathrm{~d}, J=8.2 \mathrm{~Hz}, 2 \mathrm{H}), 7.37(\mathrm{~d}, J=7.9 \mathrm{~Hz}, 1 \mathrm{H}), 7.23$ (ddd, $J=7.4,4.8,1.1 \mathrm{~Hz}, 1 \mathrm{H}) .{ }^{13} \mathrm{C} \mathrm{NMR}\left(151 \mathrm{MHz}, \mathrm{CDCl}_{3}\right) \delta 161.3,160.5,149.1,139.11,139.10,138.4,133.3$ (q, $J=32.5 \mathrm{~Hz}), 129.7,125.9(\mathrm{q}, J=3.9 \mathrm{~Hz}), 122.6,120.5 .{ }^{19} \mathrm{~F} \mathrm{NMR}\left(376 \mathrm{MHz}, \mathrm{CDCl}_{3}\right) \delta-62.9 . \mathrm{HRMS}$ $(\mathrm{ESI}+, \mathrm{m} / \mathrm{z}):[\mathrm{M}+\mathrm{H}]+$ calcd for $\mathrm{C}_{13} \mathrm{H}_{10} \mathrm{~F}_{3} \mathrm{~N}_{2}{ }^{+}: 251.0791$, found: 251.0789 .

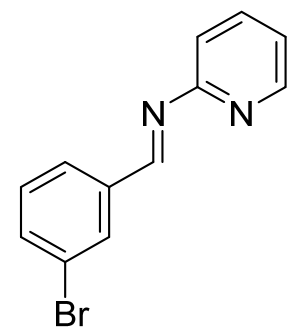

$1 e$

(E)-1-(3-Bromophenyl)-N-(pyridin-2-yl)methanimine (1e): The reaction was set up according to the General Procedure using 2-aminopyridine (188 mg, $2.00 \mathrm{mmol}, 1.0$ equiv), 3A molecular sieves (1.0 g), toluene $(4 \mathrm{~mL})$, and 3-bromobenzaldehyde ( $370 \mathrm{mg}, 2.00 \mathrm{mmol}, 1.0$ equiv). Purification by flash-column chromatography over silica gel $\left(15 \%\right.$ EtOAc/hexanes $\left.+2 \% \mathrm{Et}_{3} \mathrm{~N}\right)$ gave the desired product $1 \mathrm{e}$ as a white solid (339 mg, 65\% yield). mp 40-42 ${ }^{\circ} \mathrm{C}$. IR (neat) 3068, 1698, 1621, 1585, 1560, 1458, 1426, 1186, 788, $676 \mathrm{~cm}^{-1} .{ }^{1} \mathrm{H}$ NMR $\left(600 \mathrm{MHz}, \mathrm{CDCl}_{3}\right) \delta 9.11(\mathrm{~s}, 1 \mathrm{H}), 8.49(\mathrm{~d}, J=3.6 \mathrm{~Hz}, 1 \mathrm{H}), 8.19(\mathrm{~s}, 1 \mathrm{H}), 7.86(\mathrm{~d}, J=7.6$ 
$\mathrm{Hz}, 1 \mathrm{H}), 7.76(\mathrm{t}, J=7.6 \mathrm{~Hz}, 1 \mathrm{H}), 7.62(\mathrm{~d}, J=7.8 \mathrm{~Hz}, 1 \mathrm{H}), 7.38-7.30(\mathrm{~m}, 2 \mathrm{H}), 7.20(\mathrm{t}, J=5.9 \mathrm{~Hz}, 1 \mathrm{H}) .{ }^{13} \mathrm{C}$ NMR (151 MHz, $\left.\mathrm{CDCl}_{3}\right) \delta 161.3,160.6,149.0,138.4,138.0,134.8,131.8,130.4,128.6,123.2,122.4$, 120.3. HRMS (ESI+, $\mathrm{m} / \mathrm{z}):[\mathrm{M}+\mathrm{H}]^{+}$calcd for $\mathrm{C}_{12} \mathrm{H}_{10} \mathrm{BrN}_{2}{ }^{+}: 261.0022$, found: 261.0021 .

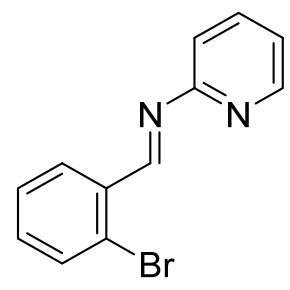

$1 f$

(E)-1-(2-Bromophenyl)-N-(pyridin-2-yl)methanimine (1f): The reaction was set up according to the General Procedure using 2-aminopyridine (188 mg, $2.00 \mathrm{mmol}, 1.0$ equiv), 3Å molecular sieves (1.0 g), toluene $(4 \mathrm{~mL})$, and 2-bromobenzaldehyde (370 mg, $2.00 \mathrm{mmol}, 1.0$ equiv). Purification by flash-column chromatography over silica gel $\left(15 \%\right.$ EtOAc/hexanes $\left.+2 \% \mathrm{Et}_{3} \mathrm{~N}\right)$ gave the desired product $1 \mathrm{f}$ as a white solid (400 mg, 77\% yield). mp 67-69 ${ }^{\circ} \mathrm{C}$. IR (neat) 1606, 1578, 1555, 1459, 1427, 1191, 786, $736 \mathrm{~cm}^{-1} .{ }^{1} \mathrm{H}$ $\operatorname{NMR}\left(600 \mathrm{MHz}, \mathrm{CDCl}_{3}\right) \delta 9.50(\mathrm{~s}, 1 \mathrm{H}), 8.53(\mathrm{~d}, J=3.8 \mathrm{~Hz}, 1 \mathrm{H}), 8.31(\mathrm{~d}, J=7.7 \mathrm{~Hz}, 1 \mathrm{H}), 7.77(\mathrm{t}, J=7.6$ $\mathrm{Hz}, 1 \mathrm{H}), 7.64(\mathrm{~d}, J=7.9 \mathrm{~Hz}, 1 \mathrm{H}), 7.41(\mathrm{t}, J=7.5 \mathrm{~Hz}, 1 \mathrm{H}), 7.37-7.31(\mathrm{~m}, 2 \mathrm{H}), 7.20(\mathrm{t}, J=5.9 \mathrm{~Hz}, 1 \mathrm{H}) .{ }^{13} \mathrm{C}$ NMR $\left(151 \mathrm{MHz}, \mathrm{CDCl}_{3}\right) \delta 162.2,161.1,149.2,138.3,134.6,133.5,133.1,129.5,127.8,127.2,122.3$, 119.9. HRMS (ESI+, m/z): [M+H] $]^{+}$calcd for $\mathrm{C}_{12} \mathrm{H}_{10} \mathrm{BrN}_{2}{ }^{+}: 261.0022$, found: 261.0020 .<smiles>Clc1ccccc1/C=N/c1ccccn1</smiles>

$1 \mathrm{~g}$

(E)-1-(2-Chlorophenyl)- $\mathrm{N}$-(pyridin-2-yl)methanimine (1g): The reaction was set up according to the General Procedure using 2-aminopyridine ( $376 \mathrm{mg}, 4.00 \mathrm{mmol}, 1.0$ equiv), $3 \AA$ molecular sieves $(2.0 \mathrm{~g})$, toluene $(8 \mathrm{~mL})$, and 2-chlorobenzaldehyde $(0.45 \mathrm{~mL}, 4.0 \mathrm{mmol}, 1.0$ equiv). Purification by flash-column chromatography over silica gel (15\% EtOAc/2\% $\mathrm{Et}_{3} \mathrm{~N} /$ hexanes) gave the desired product $1 \mathrm{~g}$ as a white solid

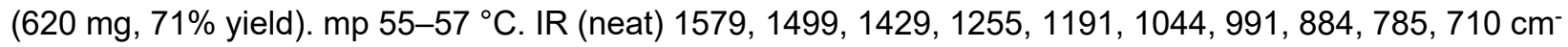
1. ${ }^{1} \mathrm{H} \mathrm{NMR}\left(400 \mathrm{MHz}, \mathrm{CDCl}_{3}\right) \delta 9.58(\mathrm{~s}, 1 \mathrm{H}), 8.53(\mathrm{dd}, J=5.2,1.9 \mathrm{~Hz}, 1 \mathrm{H}), 8.32(\mathrm{dd}, J=7.6,1.7 \mathrm{~Hz}, 1 \mathrm{H})$, $7.77(\mathrm{td}, J=7.7,1.9 \mathrm{~Hz}, 1 \mathrm{H}), 7.47-7.31(\mathrm{~m}, 4 \mathrm{H}), 7.20(\mathrm{dd}, J=7.4,4.8 \mathrm{~Hz}, 1 \mathrm{H}) .{ }^{13} \mathrm{C}$ NMR $(151 \mathrm{MHz}$, $\left.\mathrm{CDCl}_{3}\right) \delta 161.2,159.8,149.1,138.2,137.2,133.2,132.8,130.2,129.0,127.2,122.3,119.9$. HRMS (ESI+, $\mathrm{m} / \mathrm{z}):[\mathrm{M}+\mathrm{H}]+$ calcd for $\mathrm{C}_{12} \mathrm{H}_{10} \mathrm{ClN}_{2}{ }^{+}: 217.0527$, found: 217.0528 . 
<smiles>Clc1cccc(Cl)c1/C=N/c1ccccn1</smiles>

$1 \mathrm{~h}$

(E)-1-(2,6-Dichlorophenyl)-N-(pyridin-2-yl)methanimine (1h): The reaction was set up according to the General Procedure using 2-aminopyridine (188 mg, $2.00 \mathrm{mmol}, 1.0$ equiv), $3 \AA$ molecular sieves (1.0 g), toluene ( $4 \mathrm{~mL}$ ), and 2,6-dichlorobenzaldehyde ( $350 \mathrm{mg}, 2.0 \mathrm{mmol}, 1.0$ equiv). Purification by flash-column chromatography over silica gel ( $5 \% \mathrm{EtOAc} / 2 \% \mathrm{Et} 3 \mathrm{~N} / \mathrm{hexanes})$ gave the desired product $1 \mathrm{~h}$ as a white solid (389 mg, 78\% yield). mp 53-55 ${ }^{\circ} \mathrm{C}$. IR (neat) 3078, 2997, 1575, 1556, 1461, 1431, 1370, 1252, 1095, 888 $\mathrm{cm}^{-1} .{ }^{1} \mathrm{H}$ NMR $\left(500 \mathrm{MHz}, \mathrm{CDCl}_{3}\right) \delta 9.41(\mathrm{~s}, 1 \mathrm{H}), 8.54(\mathrm{dd}, J=4.8,0.9 \mathrm{~Hz}, 1 \mathrm{H}), 7.78(\mathrm{td}, J=7.7,1.9 \mathrm{~Hz}, 1 \mathrm{H})$, $7.42-7.37(\mathrm{~m}, 3 \mathrm{H}), 7.29(\mathrm{t}, J=8.0, \mathrm{~Hz}, 1 \mathrm{H}), 7.24(\mathrm{ddd}, J=7.4,4.8,1.1 \mathrm{~Hz}, 1 \mathrm{H}) .{ }^{13} \mathrm{C} \mathrm{NMR}(101 \mathrm{MHz}$, $\left.\mathrm{CDCl}_{3}\right) \delta 160.2,159.6,149.2,138.4,135.7,132.6,131.1,129.1,122.8,120.4 . \mathrm{HRMS}(\mathrm{ESI}+\mathrm{m} / \mathrm{z}):[\mathrm{M}+\mathrm{H}]$ + calcd for $\mathrm{C}_{12} \mathrm{H}_{9} \mathrm{Cl}_{2} \mathrm{~N}_{2}{ }^{+}: 251.0137$, found: 251.0136 .

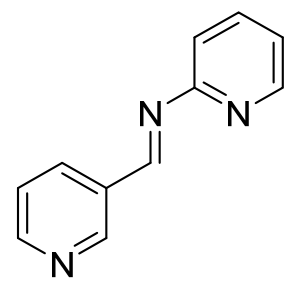

$1 \mathbf{i}$

(E)-N-(Pyridin-2-yl)-1-(pyridin-3-yl)methanimine (1i): The reaction was set up according to the General Procedure using 2-aminopyridine (188 mg, $2.00 \mathrm{mmol}, 1.0$ equiv), $3 \AA ̊$ molecular sieves $(1.0 \mathrm{~g})$, toluene (4 $\mathrm{mL})$, and 3-pyridinecarboxaldehyde $(0.19 \mathrm{~mL}, 2.0 \mathrm{mmol}, 1.0$ equiv). Purification by flash-column chromatography over silica gel ( $80 \% \mathrm{EtOAc} / 2 \% \mathrm{Et}_{3} \mathrm{~N} /$ hexanes) gave the desired product $1 \mathrm{i}$ as a white solid (243 mg, 66\% yield). mp $77-78{ }^{\circ} \mathrm{C}$. IR (neat) 3246, 3046, 1618, 1581, 1461, 1422, 1330, 1195, 889, 807, 703, $622 \mathrm{~cm}^{-1},{ }^{1} \mathrm{H}$ NMR $\left(500 \mathrm{MHz}, \mathrm{CDCl}_{3}\right) \delta 9.24(\mathrm{~s}, 1 \mathrm{H}), 9.11(\mathrm{~s}, 1 \mathrm{H}), 8.73(\mathrm{dd}, J=4.8,1.7 \mathrm{~Hz}, 1 \mathrm{H}), 8.51$ $(\mathrm{dd}, J=4.9,1.8 \mathrm{~Hz}, 1 \mathrm{H}), 8.36(\mathrm{dt}, J=8.0,1.9 \mathrm{~Hz}, 1 \mathrm{H}), 7.79(\mathrm{td}, J=7.7,1.9 \mathrm{~Hz}, 1 \mathrm{H}), 7.43(\mathrm{dd}, J=7.9,4.9$ $\mathrm{Hz}, 1 \mathrm{H}), 7.37(\mathrm{~d}, J=7.9 \mathrm{~Hz}, 1 \mathrm{H}), 7.22(\mathrm{dd}, J=7.3,4.8 \mathrm{~Hz}, 1 \mathrm{H}) .{ }^{13} \mathrm{C} \operatorname{NMR}\left(151 \mathrm{MHz}, \mathrm{CDCl}_{3}\right) \delta 160.6,160.1$, $152.6,151.8,149.1,138.4,135.6,131.7,124.0,122.6,120.4$. HRMS $(E S I+, m / z):[M+H]+$ calcd for $\mathrm{C}_{11} \mathrm{H}_{10} \mathrm{~N}_{3}{ }^{+}:$184.0869, found: 184.0871 . 
<smiles>Clc1ccc(/C=N/c2ccccn2)cn1</smiles>

$1 \mathrm{j}$

(E)-1-(6-Chloropyridin-3-yl)-N-(pyridin-2-yl)methanimine (1j): The reaction was set up according to the General Procedure using 2-aminopyridine (188 mg, $2.00 \mathrm{mmol}, 1.0$ equiv), $3 \AA$ molecular sieves (1.0 g), toluene ( $4 \mathrm{~mL})$, and 6-chloropyridine-3-carboxaldehyde ( $283 \mathrm{mg}, 2.0 \mathrm{mmol}, 1.0$ equiv). Purification by flashcolumn chromatography over silica gel (30\% EtOAc/2\% Et $3 \mathrm{~N} /$ hexanes) gave the desired product $1 \mathrm{j}$ as a white solid (229 mg, 52\% yield). $\mathrm{mp} 126-128^{\circ} \mathrm{C}$. IR (neat) 1620, 1557, 1458, 1425, 1183, 1095, 960, 888, $778,743 \mathrm{~cm}^{-1} .{ }^{1} \mathrm{H}$ NMR $\left(400 \mathrm{MHz}, \mathrm{CDCl}_{3}\right) \delta 9.23(\mathrm{~s}, 1 \mathrm{H}), 8.86(\mathrm{~d}, J=2.3 \mathrm{~Hz}, 1 \mathrm{H}), 8.51(\mathrm{dd}, J=4.8,1.9 \mathrm{~Hz}$, $1 \mathrm{H}), 8.35(\mathrm{dd}, J=8.3,2.4 \mathrm{~Hz}, 1 \mathrm{H}), 7.79(\mathrm{td}, J=7.7,1.9 \mathrm{~Hz}, 1 \mathrm{H}), 7.46(\mathrm{~d}, J=8.3 \mathrm{~Hz}, 1 \mathrm{H}), 7.36(\mathrm{~d}, J=7.9$ $\mathrm{Hz}, 1 \mathrm{H}$ ), 7.24 (ddd, $J=7.4,4.8,1.1 \mathrm{~Hz}, 1 \mathrm{H}) .{ }^{13} \mathrm{C}$ NMR $\left(151 \mathrm{MHz}, \mathrm{CDCl}_{3}\right) \delta 160.2,158.6,154.4,151.7$, $149.1,138.5,138.0,130.7,124.9,122.8,120.6$. HRMS $(E S I+, m / z):[M+H]+$ calcd for $\mathrm{C}_{11} \mathrm{H}_{9} \mathrm{ClN}_{3}{ }^{+}$: 218.0480, found: 218.0480 .

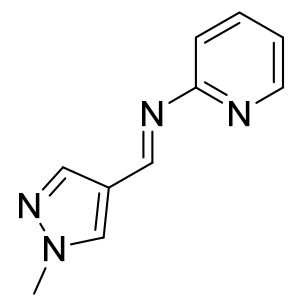

$1 \mathrm{k}$

(E)-1-(1-Methyl-1H-pyrazol-4-yl)-N-(pyridin-2-yl)methanimine (1k): The reaction was set up according to the General Procedure using 2-aminopyridine ( $376 \mathrm{mg}, 4.00 \mathrm{mmol}, 1.0$ equiv), $3 \AA$ molecular sieves (2.0 $\mathrm{g}$ ), toluene $(8.0 \mathrm{~mL}$ ), and 1-methylpyrazole-4-carbaldehyde (485 mg, $4.40 \mathrm{mmol}, 1.1 \mathrm{equiv})$. Recrystallization ( $\mathrm{Et}_{2} \mathrm{O} /$ pentane) gave the desired product $1 \mathrm{k}$ as a white solid $(69.0 \mathrm{mg}, 9 \%$ yield). mp 68$70{ }^{\circ} \mathrm{C}$. IR (neat) 3232, 1681, 1618, 1586, 1560, 1428, 1160, 877, $776 \mathrm{~cm}^{-1} .{ }^{1} \mathrm{H} \mathrm{NMR}\left(400 \mathrm{MHz}, \mathrm{CDCl}_{3}\right) \delta$ $9.08(\mathrm{~s}, 1 \mathrm{H}), 8.45$ (dd, J = 4.9, 1.2 Hz, 1H), $8.00(\mathrm{~s}, 1 \mathrm{H}), 7.91(\mathrm{~s}, 1 \mathrm{H}), 7.72(\mathrm{td}, J=7.7,1.9 \mathrm{~Hz}, 1 \mathrm{H}), 7.25$ $(\mathrm{d}, J=7.5 \mathrm{~Hz}, 1 \mathrm{H}), 7.17-7.12(\mathrm{~m}, 1 \mathrm{H}), 3.97(\mathrm{~s}, 3 \mathrm{H}) .{ }^{13} \mathrm{C}$ NMR $\left(151 \mathrm{MHz}, \mathrm{CDCl}_{3}\right) \delta 161.4,154.9,148.9$, 140.7, 138.3, 132.1, 121.64, 121.60, 119.6, 39.5. HRMS (ESI+, m/z): $[\mathrm{M}+\mathrm{H}]^{+}$calcd $\mathrm{C}_{10} \mathrm{H}_{11} \mathrm{~N}_{4}^{+}: 187.0978$, found: 187.0978 . 


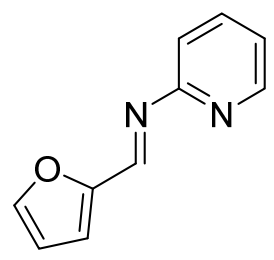

11

(E)-1-(Furan-2-yl)-N-(pyridin-2-yl)methanimine (1I): ${ }^{8}$ The reaction was set up according to the General Procedure using 2-aminopyridine (188 mg, $2.00 \mathrm{mmol}, 1.0$ equiv), 3Å molecular sieves (1.0 g), toluene (4 $\mathrm{mL}$ ), and furfural (192 mg, $2.00 \mathrm{mmol}, 1.0$ equiv). Purification by flash-column chromatography over silica

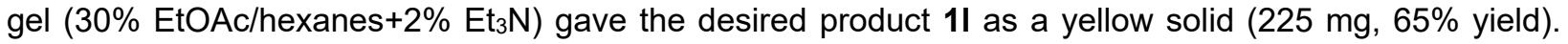
Spectroscopic data are in agreement with reported values. ${ }^{8}{ }^{1} \mathrm{H} \mathrm{NMR}\left(600 \mathrm{MHz}, \mathrm{CDCl}_{3}\right) \delta 9.11(\mathrm{~s}, 1 \mathrm{H}), 8.46$ $(\mathrm{d}, J=3.7 \mathrm{~Hz}, 1 \mathrm{H}), 7.74(\mathrm{t}, J=7.6 \mathrm{~Hz}, 1 \mathrm{H}), 7.66(\mathrm{~s}, 1 \mathrm{H}), 7.41(\mathrm{~d}, J=7.9 \mathrm{~Hz}, 1 \mathrm{H}), 7.17(\mathrm{t}, J=5.8 \mathrm{~Hz}, 1 \mathrm{H})$, $7.06(\mathrm{~d}, J=2.2 \mathrm{~Hz}, 1 \mathrm{H}), 6.58(\mathrm{~s}, 1 \mathrm{H}) .{ }^{13} \mathrm{C}$ NMR $\left(151 \mathrm{MHz}, \mathrm{CDCl}_{3}\right) \delta 160.2,152.3,149.8,148.8,146.5$, 138.4, 122.3, 121.1, 119.0, 112.6.

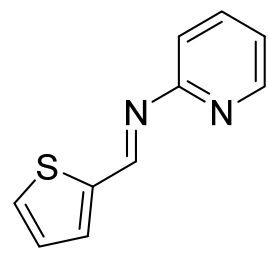

$1 \mathrm{~m}$

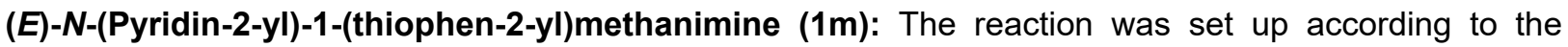
General Procedure using 2-aminopyridine (188 mg, $2.00 \mathrm{mmol}, 1.0$ equiv), $3 \AA$ molecular sieves $(1.0 \mathrm{~g})$, toluene $(4 \mathrm{~mL})$, and 2-thiophenecarboxaldehyde $(224 \mathrm{mg}, 2.00 \mathrm{mmol}, 1.0$ equiv). Purification by flashcolumn chromatography over silica gel $\left(15 \% \mathrm{EtOAc} /\right.$ hexanes $\left.+2 \% \mathrm{Et}_{3} \mathrm{~N}\right)$ gave the desired product $1 \mathrm{~m}$ as a white solid (314 mg, 84\% yield). mp 56-57 ${ }^{\circ} \mathrm{C}$. IR (neat) 1604, 1578, 1553, 1415, 1197, 789, $718 \mathrm{~cm}^{-1} .{ }^{1} \mathrm{H}$ NMR $\left(600 \mathrm{MHz}, \mathrm{CDCl}_{3}\right) \delta 9.34(\mathrm{~s}, 1 \mathrm{H}), 8.47(\mathrm{~d}, J=4.6 \mathrm{~Hz}, 1 \mathrm{H}), 7.74(\mathrm{t}, J=7.6 \mathrm{~Hz}, 1 \mathrm{H}), 7.60(\mathrm{~d}, J=3.4$ $\mathrm{Hz}, 1 \mathrm{H}), 7.56(\mathrm{~d}, J=4.9 \mathrm{~Hz}, 1 \mathrm{H}), 7.33(\mathrm{~d}, J=7.9 \mathrm{~Hz}, 1 \mathrm{H}), 7.21-7.10(\mathrm{~m}, 2 \mathrm{H}) .{ }^{13} \mathrm{C} \mathrm{NMR}\left(151 \mathrm{MHz}, \mathrm{CDCl}_{3}\right)$ $\delta 160.5,155.6,148.9,142.7,138.3,134.2,131.4,128.2,122.1,120.5$. HRMS (ESI+, m/z): $[\mathrm{M}+\mathrm{H}]^{+}$calcd $\mathrm{C}_{10} \mathrm{H}_{9} \mathrm{~N}_{2} \mathrm{~S}^{+}:$: 189.0481 , found: 189.0482 .<smiles>COC(=O)c1ccc(/C=N/c2ccccn2)cc1</smiles>

$1 \mathrm{n}$ 
Methyl (E)-4-((pyridin-2-ylimino)methyl)benzoate (1n): The reaction was set up according to the General Procedure using 2-aminopyridine (188 mg, $2.00 \mathrm{mmol}, 1.0$ equiv), $3 \AA$ molecular sieves $(1.0 \mathrm{~g})$, toluene (4 $\mathrm{mL}$ ), and methyl 4-formylbenzoate (328 mg, $2.0 \mathrm{mmol}, 1.0$ equiv). Purification by flash-column chromatography over silica gel (20\% EtOAc/2\% Et 3 N/hexanes) gave the desired product $1 \mathrm{n}$ as a white solid (363 mg, 75\% yield). mp 130-131 ${ }^{\circ} \mathrm{C}$. IR (neat) 3079, 2952, 1711, 1556, 1434, 1276, 1109, 793, 698, 544 $\mathrm{cm}^{-1} .{ }^{1} \mathrm{H}$ NMR $\left(400 \mathrm{MHz}, \mathrm{CDCl}_{3}\right) \delta 9.22(\mathrm{~s}, 1 \mathrm{H}), 8.51(\mathrm{dd}, J=4.9,1.9 \mathrm{~Hz}, 1 \mathrm{H}), 8.14(\mathrm{~d}, J=8.3 \mathrm{~Hz}, 2 \mathrm{H}), 8.05$ $(\mathrm{d}, J=8.3 \mathrm{~Hz}, 2 \mathrm{H}), 7.78(\mathrm{td}, J=7.7,1.9 \mathrm{~Hz}, 1 \mathrm{H}), 7.37$ (d, $J=7.9 \mathrm{~Hz}, 1 \mathrm{H}), 7.21$ (ddd, $J=7.4,4.8,1.1 \mathrm{~Hz}$, $1 \mathrm{H}), 3.95$ (s, 3H). ${ }^{13} \mathrm{C}$ NMR (151 MHz, $\left.\mathrm{CDCl}_{3}\right) \delta 166.7,161.8,160.7,149.1,139.8,138.4,132.9,130.1$, 129.4, 122.5, 120.4, 52.5. HRMS (ESI+, m/z): $[\mathrm{M}+\mathrm{H}]+$ calcd for $\mathrm{C}_{14} \mathrm{H}_{13} \mathrm{~N}_{2} \mathrm{O}_{2}{ }^{+}: 241.0972$, found: 241.0971 .

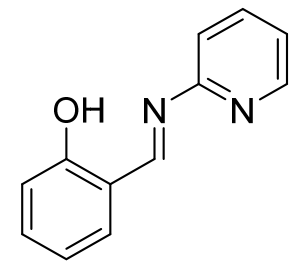

10

(E)-2-((Pyridin-2-ylimino)methyl)phenol (10): ${ }^{7}$ The reaction was set up according to the General Procedure using 2-aminopyridine (188 mg, $2.00 \mathrm{mmol}, 1.0$ equiv), $3 \AA$ molecular sieves ( $1.0 \mathrm{~g})$, toluene (4 $\mathrm{mL})$, and 2-hydroxybenzaldehyde $(0.21 \mathrm{~mL}, 2.0 \mathrm{mmol}, 1.0$ equiv). Purification by flash-column chromatography over silica gel ( $5 \% \mathrm{EtOAc} / 2 \% \mathrm{Et}_{3} \mathrm{~N} /$ hexanes) gave the desired product 10 as a yellow solid (221 mg, 56\% yield). Reported spectra match literature data. ${ }^{7}{ }^{1} \mathrm{H} \mathrm{NMR}\left(500 \mathrm{MHz}, \mathrm{CDCl}_{3}\right) \delta 13.44(\mathrm{~s}, 1 \mathrm{H})$, $9.45(\mathrm{~s}, 1 \mathrm{H}), 8.51$ (dd, $J=4.6,2.3 \mathrm{~Hz}, 1 \mathrm{H}), 7.78(\mathrm{t}, J=7.7 \mathrm{~Hz}, 1 \mathrm{H}), 7.51(\mathrm{~d}, J=7.7 \mathrm{~Hz}, 1 \mathrm{H}), 7.41(\mathrm{t}, J=7.8$ $\mathrm{Hz}, 1 \mathrm{H}), 7.33(\mathrm{~d}, J=7.9 \mathrm{~Hz}, 1 \mathrm{H}), 7.25-7.20(\mathrm{~m}, 1 \mathrm{H}), 7.03(\mathrm{~d}, J=8.4 \mathrm{~Hz}, 1 \mathrm{H}), 6.96(\mathrm{t}, J=7.5 \mathrm{~Hz}, 1 \mathrm{H}) .{ }^{13} \mathrm{C}$ NMR $\left(151 \mathrm{MHz}, \mathrm{CDCl}_{3}\right) \delta 164.9,162.0,157.7,149.1,138.6,134.0,133.6,122.7,120.6,119.4,119.1$, 117.4.

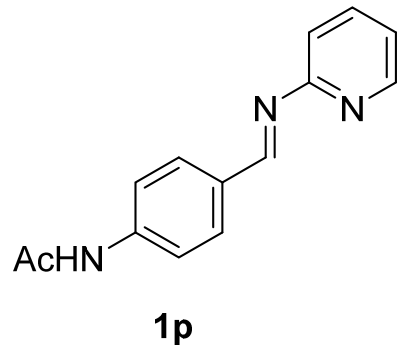

(E)-N-(4-((Pyridin-2-ylimino)methyl)phenyl)acetamide (1p): The reaction was set up according to a modified version of the General Procedure using 2-aminopyridine ( $376 \mathrm{mg}, 4.00 \mathrm{mmol}, 1.0 \mathrm{equiv}$ ), $3 \AA$ molecular sieves $(2.0 \mathrm{~g})$, toluene $(8.0 \mathrm{~mL})$, and 4-acetamidobenzaldehyde (653 mg, $4.00 \mathrm{mmol}, 1.0$ equiv). The reaction mixture was stirred for $48 \mathrm{~h}$ at $80^{\circ} \mathrm{C}$. The toluene was then filtered off and discarded. The remaining material was dissolved in $\mathrm{CH}_{2} \mathrm{Cl}_{2}$ and filtered. Recrystallization (EtOAc/hexanes) gave the desired product $1 \mathrm{p}$ as a pale-yellow solid (478 mg, $50 \%$ yield). $\mathrm{mp} 144-146{ }^{\circ} \mathrm{C}$. IR (neat) 3247,3045 , 1668, 1581, 1525, 1320, 1166, 841, 786, $736 \mathrm{~cm}^{-1} .{ }^{1} \mathrm{H} \mathrm{NMR}\left(400 \mathrm{MHz}, \mathrm{CDCl}_{3}\right) \delta 9.06$ (s, 1H), 8.48 (dd, $J$ $=5.0,1.1 \mathrm{~Hz}, 1 \mathrm{H}), 7.93(\mathrm{~d}, J=8.6 \mathrm{~Hz}, 2 \mathrm{H}), 7.75(\mathrm{td}, J=7.8,1.9 \mathrm{~Hz}, 1 \mathrm{H}$ ), 7.64 (overlapping d, $J=8.3 \mathrm{~Hz}$, $3 \mathrm{H}), 7.30(\mathrm{~d}, J=8.0 \mathrm{~Hz}, 1 \mathrm{H}), 7.21-7.14(\mathrm{~m}, 1 \mathrm{H}), 2.20(\mathrm{~s}, 3 \mathrm{H}) .{ }^{13} \mathrm{C} \mathrm{NMR}\left(151 \mathrm{MHz}, \mathrm{CDCl}_{3}\right) \delta 168.5,162.1$, 
161.4, 149.0, 141.4, 138.3, 131.9, 130.7, 121.9, 119.8, 119.5, 24.9. HRMS (ESI+, m/z): $[\mathrm{M}+\mathrm{H}]^{+}$calcd for $\mathrm{C}_{14} \mathrm{H}_{14} \mathrm{~N}_{3} \mathrm{O}^{+}: 240.1131$, found: 240.1128 .

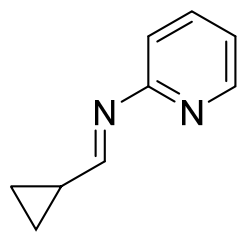

$1 q$

(E)-1-Cyclopropyl-N-(pyridin-2-yl)methanimine (1q): To a 25-mL round-bottom flask was added 2aminopyridine (376 mg, $4.00 \mathrm{mmol}, 1.0$ equiv), $3 \AA$ molecular sieves $(2.0 \mathrm{~g})$, toluene $(8.0 \mathrm{~mL})$, and cyclopropanecarboxaldehyde $\left(0.90 \mathrm{~mL}, 12 \mathrm{mmol}, 3.0\right.$ equiv). The reaction solution was stirred under $\mathrm{N}_{2}$ at $80{ }^{\circ} \mathrm{C}$ for $48 \mathrm{~h}$. The reaction mixture was filtered in a $\mathrm{N}_{2}$-filled glovebox and concentrated under a stream of $\mathrm{N}_{2}$ to yield the desired product $1 \mathrm{q}$ as a yellow oil (336 mg, 58\% yield). IR (neat) 3005, 1634, 1586, 1561, 1460, 1432, 942, 785, $741 \mathrm{~cm}^{-1} .{ }^{1} \mathrm{H}$ NMR $\left(400 \mathrm{MHz}, \mathrm{CDCl}_{3}\right) \delta 8.38(\mathrm{dd}, J=4.9,1.0 \mathrm{~Hz}, 1 \mathrm{H}), 7.88(\mathrm{~d}, J=$ $8.2 \mathrm{~Hz}, 1 \mathrm{H}), 7.68(\mathrm{td}, J=7.8,1.9 \mathrm{~Hz}, 1 \mathrm{H}), 7.19-7.05(\mathrm{~m}, 2 \mathrm{H}), 1.99-1.88(\mathrm{~m}, 1 \mathrm{H}), 1.13-1.06(\mathrm{~m}, 2 \mathrm{H})$, $1.02-0.95(\mathrm{~m}, 2 \mathrm{H}) .{ }^{13} \mathrm{C} \mathrm{NMR}\left(151 \mathrm{MHz}, \mathrm{CDCl}_{3}\right) \delta 172.1,161.0,148.8,138.2,121.4,118.8,18.0,7.9$. HRMS (ESI+, m/z): [M+H] $]^{+}$calcd for $\mathrm{C}_{9} \mathrm{H}_{11} \mathrm{~N}_{2}{ }^{+}: 147.0917$, found: 147.0917 .

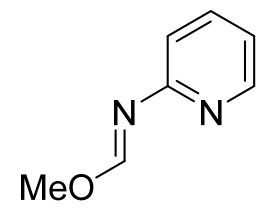

$1 \mathrm{r}$

Methyl (E)-N-(pyridin-2-yl)formimidate (1r): To a 25-mL round-bottom flask was added 2-aminopyridine (376 mg, $4.00 \mathrm{mmol}, 1.0$ equiv), trimethyl orthoformate $(12 \mathrm{~mL})$ and trifluoroacetic acid $(0.03 \mathrm{~mL}, 0.4 \mathrm{mmol}$, 0.1 equiv). The reaction mixture was stirred at reflux overnight. The crude mixture was then concentrated in vacuo and purified by flash-column chromatography over silica gel (30\% EtOAc/hexanes $+2 \% \mathrm{Et} 3 \mathrm{~N})$ to give the desired product $1 \mathrm{r}$ as a clear oil (198 mg, 36\% yield). IR (neat) 3001, 2945, 16291588,1561 , 1463, 1438, 1278, 1200, 923, 786, 742, $701 \mathrm{~cm}^{-1} .{ }^{1} \mathrm{H}$ NMR $\left(400 \mathrm{MHz}, \mathrm{CDCl}_{3}\right) \delta 8.36$ (dd, $J=5.0,0.8 \mathrm{~Hz}$, 1H), $8.32(\mathrm{~s}, 1 \mathrm{H}), 7.65$ (td, $J=7.7,2.0 \mathrm{~Hz}, 1 \mathrm{H}), 7.11-7.04(\mathrm{~m}, 1 \mathrm{H}), 7.01(\mathrm{~d}, J=8.0 \mathrm{~Hz}, 1 \mathrm{H}), 3.93(\mathrm{~s}, 3 \mathrm{H})$. ${ }^{13} \mathrm{C}$ NMR $\left(151 \mathrm{MHz}, \mathrm{CDCl}_{3}\right) \delta 159.5,159.0,148.8,138.2,120.4,118.1,54.3 . \mathrm{HRMS}(\mathrm{ESI}+\mathrm{m} / \mathrm{z}):[\mathrm{M}+\mathrm{H}]^{+}$ calcd for $\mathrm{C}_{7} \mathrm{H}_{9} \mathrm{~N}_{2} \mathrm{O}^{+}$: 137.0709, found: 137.0708. 


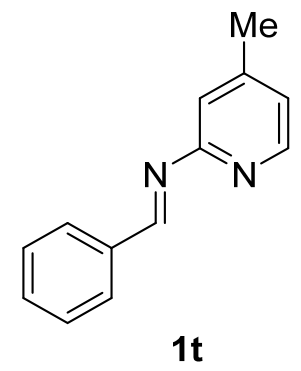

(E)-N-(4-Methylpyridin-2-yl)-1-phenylmethanimine (1t): The reaction was set up according to the

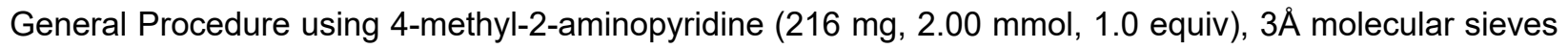
$(1.0 \mathrm{~g})$, toluene $(4 \mathrm{~mL})$, and benzaldehyde $(0.20 \mathrm{~mL}, 2.0 \mathrm{mmol}, 1.0$ equiv). Purification by flash-column chromatography over silica gel $\left(10 \% \mathrm{EtOAc} / 2 \% \mathrm{Et}_{3} \mathrm{~N} /\right.$ hexanes) gave the desired product $1 \mathrm{t}$ as a yellow oil (250 mg, 64\% yield). $\mathrm{mp} 62-63^{\circ} \mathrm{C}$. IR (neat) 1593, 1545, 1449, 1404, 1214, 1145, 995, 895, 827, 740, $688,487 \mathrm{~cm}^{-1} .{ }^{1} \mathrm{H}$ NMR $\left(400 \mathrm{MHz}, \mathrm{CDCl}_{3}\right) \delta 9.15(\mathrm{~s}, 1 \mathrm{H}), 8.35(\mathrm{~d}, J=5.0 \mathrm{~Hz}, 1 \mathrm{H}), 7.99(\mathrm{dd}, J=7.6,1.6 \mathrm{~Hz}$, $2 \mathrm{H}), 7.55-7.44(\mathrm{~m}, 3 \mathrm{H}), 7.17(\mathrm{~s}, 1 \mathrm{H}), 7.01(\mathrm{~d}, J=5.0 \mathrm{~Hz}, 1 \mathrm{H}), 2.40(\mathrm{~s}, 3 \mathrm{H}) .{ }^{13} \mathrm{C} \mathrm{NMR}\left(151 \mathrm{MHz}, \mathrm{CDCl}_{3}\right) \delta$ $162.9,161.3,149.6,148.7,136.1,132.0,129.6,128.9,123.1,120.6,21.1$. HRMS (ESI+, m/z): $[M+H]+$ calcd for $\mathrm{C}_{13} \mathrm{H}_{13} \mathrm{~N}_{2}{ }^{+}:$197.1073, found: 197.1073.<smiles>Cc1ccc(/N=C/c2ccccc2)nc1</smiles>

1u

(E)-N-(5-Methylpyridin-2-yl)-1-phenylmethanimine (1u): The reaction was set up according to the General Procedure using 5-methyl-2-aminopyridine $(216 \mathrm{mg}, 2.00 \mathrm{mmol}, 1.0$ equiv), $3 \AA$ molecular sieves $(1.0 \mathrm{~g})$, toluene $(4 \mathrm{~mL})$, and benzaldehyde $(0.20 \mathrm{~mL}, 2.0 \mathrm{mmol}, 1.0$ equiv). Purification by flash-column chromatography over silica gel (10\% EtOAc/2\% Et 3 N/hexanes) gave the desired product $1 \mathrm{u}$ as a white solid (318 mg, 81\% yield). mp 71-72 ${ }^{\circ} \mathrm{C}$. IR (neat) 1618, 1574, 1469, 1193, 995, 838, 762, 689, $538 \mathrm{~cm}^{-1} .{ }^{1} \mathrm{H}$ $\operatorname{NMR}\left(400 \mathrm{MHz}, \mathrm{CDCl}_{3}\right) \delta 9.18(\mathrm{~s}, 1 \mathrm{H}), 8.33(\mathrm{~d}, J=2.3 \mathrm{~Hz}, 1 \mathrm{H}), 8.00(\mathrm{dd}, J=7.6,1.7 \mathrm{~Hz}, 2 \mathrm{H}), 7.58(\mathrm{dd}, J$ $=8.1,2.4 \mathrm{~Hz}, 1 \mathrm{H}), 7.54-7.45(\mathrm{~m}, 3 \mathrm{H}), 7.28(\mathrm{~s}, 1 \mathrm{H}), 3.14(\mathrm{~s}, 3 \mathrm{H}) .{ }^{13} \mathrm{C}$ NMR $\left(151 \mathrm{MHz}, \mathrm{CDCl}_{3}\right) \delta 162.1$, 159.0, 149.2, 138.9, 136.2, 131.9, 131.6, 129.5, 128.9, 119.6, 18.2. HRMS (ESI+, m/z): $[\mathrm{M}+\mathrm{H}]+$ calcd for $\mathrm{C}_{13} \mathrm{H}_{13} \mathrm{~N}_{2}{ }^{+}:$197.1073, found: 197.1073 .<smiles>Cc1cccc(/N=C/c2ccccc2)n1</smiles>

$1 \mathrm{v}$ 
(E)-N-(6-Methylpyridin-2-yl)-1-phenylmethanimine (1v): The reaction was set up according to the General Procedure using 6-methyl-2-aminopyridine ( $476 \mathrm{mg}, 4.00 \mathrm{mmol}, 1.0$ equiv), $3 \AA$ molecular sieves $(2.0 \mathrm{~g})$, toluene $(8 \mathrm{~mL})$, and benzaldehyde $(0.40 \mathrm{~mL}, 4.0 \mathrm{mmol}, 1.0$ equiv). Purification by flash-column chromatography over silica gel ( $5 \% \mathrm{EtOAc} / 2 \% \mathrm{Et}_{3} \mathrm{~N} /$ hexanes) gave the desired product $1 \mathrm{v}$ as a colorless oil which solidified upon freezing (538 mg, $69 \%$ yield). $\mathrm{mp} 57-59{ }^{\circ} \mathrm{C}$. IR (neat) $1614,1556,1448,1192$, 1152, 761, 732, 689, $545 \mathrm{~cm}^{-1} .{ }^{1} \mathrm{H}$ NMR $\left(400 \mathrm{MHz}, \mathrm{CDCl}_{3}\right) \delta 9.07(\mathrm{~s}, 1 \mathrm{H}), 7.99(\mathrm{dd}, J=7.4,2.1 \mathrm{~Hz}, 2 \mathrm{H})$, $7.64(\mathrm{t}, J=7.7 \mathrm{~Hz}, 1 \mathrm{H}), 7.51-7.45(\mathrm{~m}, 3 \mathrm{H}), 7.10(\mathrm{~d}, J=7.8 \mathrm{~Hz}, 1 \mathrm{H}), 7.05(\mathrm{~d}, J=7.5 \mathrm{~Hz}, 1 \mathrm{H}), 2.58(\mathrm{~s}, 3 \mathrm{H})$. ${ }^{13} \mathrm{C}$ NMR $\left(151 \mathrm{MHz}, \mathrm{CDCl}_{3}\right) \delta 162.8,161.1,158.0,138.4,136.1,131.9,129.6,128.8,121.4,115.9,24.6$. HRMS (ESI+, m/z): [M+H] + calcd for $\mathrm{C}_{13} \mathrm{H}_{13} \mathrm{~N}_{2}{ }^{+}:$197.1073, found: 197.1073.<smiles>Clc1ccc(/N=C/c2ccccc2)nc1</smiles>

$1 w$

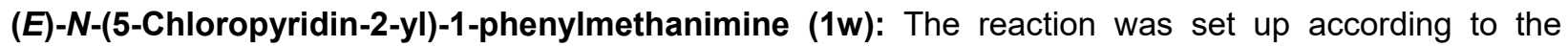
General Procedure using 5-chloro-2-aminopyridine (257 mg, $2.00 \mathrm{mmol}, 1.0$ equiv), 3A molecular sieves $(1.0 \mathrm{~g})$, toluene $(4 \mathrm{~mL})$, and benzaldehyde $(0.20 \mathrm{~mL}, 2.0 \mathrm{mmol}, 1.0$ equiv). Purification by flash-column chromatography over silica gel (10\% EtOAc/2\% $\mathrm{Et}_{3} \mathrm{~N} /$ hexanes) gave the desired product $1 \mathbf{w}$ as a white solid (318 mg, 73\% yield). mp 83-84 ${ }^{\circ} \mathrm{C}$. IR (neat) 1615, 1570, 1446, 1380, 1194, 1105, 1004, 854, 831, 764, $686 \mathrm{~cm}^{-1} .{ }^{1} \mathrm{H}$ NMR $\left(600 \mathrm{MHz}, \mathrm{CDCl}_{3}\right) \delta 9.14(\mathrm{~s}, 1 \mathrm{H}), 8.44(\mathrm{~s}, 1 \mathrm{H}), 7.98(\mathrm{~d}, J=7.2 \mathrm{~Hz}, 2 \mathrm{H}), 7.72(\mathrm{dd}, J$ $=8.5,2.5 \mathrm{~Hz}, 1 \mathrm{H}), 7.54-7.47(\mathrm{~m}, 3 \mathrm{H}), 7.30(\mathrm{~d}, J=8.4 \mathrm{~Hz}, 1 \mathrm{H}) .{ }^{13} \mathrm{C} \mathrm{NMR}\left(151 \mathrm{MHz}, \mathrm{CDCl}_{3}\right) \delta$ 163.5, 159.5, 147.7, 137.9, 135.8, 132.3, 129.9, 129.7, 129.0, 120.9. HRMS $(\mathrm{ESI}+, \mathrm{m} / \mathrm{z}):[\mathrm{M}+\mathrm{H}]+$ calcd for $\mathrm{C}_{12} \mathrm{H}_{10} \mathrm{ClN}_{2}{ }^{+}$: 217.0527, found: 217.0527 .<smiles>FC(F)(F)c1ccnc(/N=C/c2ccccc2)c1</smiles>

$1 x$

(E)-1-Phenyl-N-(4-(trifluoromethyl)pyridin-2-yl)methanimine (1x): ${ }^{9}$ The reaction was set up according to the General Procedure using 4-(trifluoromethyl)pyridin-2-amine (324 mg, $2.00 \mathrm{mmol}, 1.0 \mathrm{equiv}$ ), $3 \AA$ molecular sieves $(1.0 \mathrm{~g})$, toluene $(4 \mathrm{~mL})$, and benzaldehyde $(0.20 \mathrm{~mL} 2.0 \mathrm{mmol}, 1.0$ equiv). Purification by flash-column chromatography over silica gel $\left(20 \% \mathrm{EtOAc} /\right.$ hexanes $\left.+2 \% \mathrm{Et}_{3} \mathrm{~N}\right)$ gave the desired product $\mathbf{1 x}$ as a white solid (313 mg, 63\% yield). Spectroscopic data are in agreement with reported values. ${ }^{9}{ }^{1} \mathrm{H}$ NMR $\left(600 \mathrm{MHz}, \mathrm{CDCl}_{3}\right) \delta 9.17(\mathrm{~s}, 1 \mathrm{H}), 8.66(\mathrm{~d}, J=5.1 \mathrm{~Hz}, 1 \mathrm{H}), 8.01(\mathrm{~d}, J=7.0 \mathrm{~Hz}, 2 \mathrm{H}), 7.59-7.53(\mathrm{~m}, 2 \mathrm{H})$, $7.53-7.48(\mathrm{~m}, 2 \mathrm{H}), 7.40(\mathrm{~d}, J=5.0 \mathrm{~Hz}, 1 \mathrm{H}) .{ }^{13} \mathrm{C} \mathrm{NMR}\left(151 \mathrm{MHz}, \mathrm{CDCl}_{3}\right) \delta$ 164.6, 162.2, 150.1, 140.6 (q, 
$J=34.1 \mathrm{~Hz}), 135.6,132.7,129.9,129.0,122.9(\mathrm{q}, J=273.2 \mathrm{~Hz}), 117.4(\mathrm{q}, J=3.4 \mathrm{~Hz}), 116.1(\mathrm{q}, J=3.7$ $\mathrm{Hz}) .{ }^{19} \mathrm{~F} \mathrm{NMR}\left(376 \mathrm{MHz}, \mathrm{CDCl}_{3}\right) \delta-64.9$.<smiles>Cc1ccc(/N=C/c2ccccc2)nn1</smiles>

$1 y$

(E)-N-(6-Methylpyridazin-3-yl)-1-phenylmethanimine (1y): The reaction was set up according to the General Procedure using 6-methylpyridazin-3-amine (218 mg, $2.00 \mathrm{mmol}, 1.0$ equiv), $3 \AA$ molecular sieves $(1.0 \mathrm{~g})$, toluene $(4 \mathrm{~mL})$, and benzaldehyde $(0.20 \mathrm{~mL}, 2.0 \mathrm{mmol}, 1.0$ equiv). Purification by flash-column chromatography over silica gel $\left(50 \%\right.$ EtOAc/hexanes $\left.+2 \% \mathrm{Et}_{3} \mathrm{~N}\right)$ gave the desired product $1 \mathrm{y}$ as a paleyellow solid (210 mg, 53\% yield). $\mathrm{mp} 91-93^{\circ} \mathrm{C}$. IR (neat) 3059, 1621, 1574, 1411, 1201, 855, 748, 691 $\mathrm{cm}^{-1} .{ }^{1} \mathrm{H}$ NMR $\left(400 \mathrm{MHz}, \mathrm{CDCl}_{3}\right) \delta 9.20(\mathrm{~s}, 1 \mathrm{H}), 8.00(\mathrm{dd}, J=7.9,1.4 \mathrm{~Hz}, 2 \mathrm{H}), 7.57-7.47(\mathrm{~m}, 3 \mathrm{H}), 7.39$ $(\mathrm{d}, J=9.2 \mathrm{~Hz}, 1 \mathrm{H}), 7.36(\mathrm{~d}, J=9.1 \mathrm{~Hz}, 1 \mathrm{H}), 2.73(\mathrm{~s}, 3 \mathrm{H}) .{ }^{13} \mathrm{C} \mathrm{NMR}\left(101 \mathrm{MHz}, \mathrm{CDCl}_{3}\right) \delta 164.4,162.1$, 158.6, 135.7, 132.5, 129.9, 129.0, 128.7, 123.8, 22.0. HRMS (ESI+, m/z): $[\mathrm{M}+\mathrm{H}]^{+}$calcd for $\mathrm{C}_{12} \mathrm{H}_{12} \mathrm{~N}_{3}{ }^{+}$: 198.1026, found: 198.1028 .<smiles>Cn1ccnc1/N=C/c1ccccc1</smiles>

(E)-N-(1-Methyl-1H-imidazol-2-yl)-1-phenylmethanimine (1z): ${ }^{10}$ The reaction was set up according to literature procedure. ${ }^{10}$ Purification by flash-column chromatography over silica gel ( $40 \%$ acetone/hexanes) gave the desired product $1 \mathrm{z}$ as a yellow oil (194 mg, $69 \%$ yield). Reported spectra match literature data. ${ }^{10}$ ${ }^{1} \mathrm{H} \mathrm{NMR}\left(500 \mathrm{MHz}, \mathrm{CDCl}_{3}\right) \delta 9.22(\mathrm{~s}, 1 \mathrm{H}), 7.95(\mathrm{~d}, J=7.8 \mathrm{~Hz}, 2 \mathrm{H}), 7.52-7.38(\mathrm{~m}, 3 \mathrm{H}), 7.01(\mathrm{~d}, J=2.4 \mathrm{~Hz}$, $1 \mathrm{H}), 6.91(\mathrm{~d}, J=2.3 \mathrm{~Hz}, 1 \mathrm{H}), 3.78(\mathrm{~s}, 3 \mathrm{H}) .{ }^{13} \mathrm{C} \mathrm{NMR}\left(151 \mathrm{MHz}, \mathrm{CDCl}_{3}\right) \delta 159.9,150.3,136.2,131.8,129.2$, $128.9,127.3,120.5,31.7$.<smiles>Cn1c(/N=C/c2ccccc2)nc2ccccc21</smiles>

1 aa 
(E)-N-(1-Methyl-1H-benzo[d]imidazol-2-yl)-1-phenylmethanimine (1aa): The reaction was set up according to the General Procedure using amine ( $294 \mathrm{mg}, 2.00 \mathrm{mmol}, 1.0$ equiv), $3 \AA$ molecular sieves ( 1.0 $\mathrm{g})$, toluene $(4.0 \mathrm{~mL})$, and benzaldehyde $(0.20 \mathrm{~mL}, 2.0 \mathrm{mmol}, 1.0$ equiv). Purification by flash-column

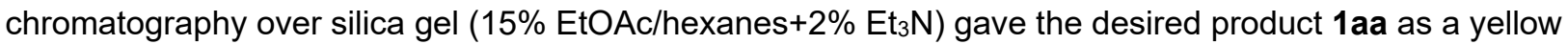
solid (404 mg, 86\% yield). mp 98-99 ${ }^{\circ} \mathrm{C}$. IR (neat) 3050, 1606, 1573, 1462, 1390, 764, 742, 706, $687 \mathrm{~cm}^{-}$ 1. ${ }^{1} \mathrm{H}$ NMR $\left(600 \mathrm{MHz}, \mathrm{CDCl}_{3}\right) \delta 9.53(\mathrm{~s}, 1 \mathrm{H}), 8.06(\mathrm{~d}, J=7.1 \mathrm{~Hz}, 2 \mathrm{H}), 7.82-7.65(\mathrm{~m}, 1 \mathrm{H}), 7.56(\mathrm{t}, J=7.2$ $\mathrm{Hz}, 1 \mathrm{H}), 7.52(\mathrm{t}, J=7.4 \mathrm{~Hz}, 2 \mathrm{H}), 7.40-7.34(\mathrm{~m}, 1 \mathrm{H}), 7.32-7.27(\mathrm{~m}, 2 \mathrm{H}), 3.94(\mathrm{~s}, 3 \mathrm{H}) .{ }^{13} \mathrm{C}$ NMR $(151$ $\left.\mathrm{MHz}, \mathrm{CDCl}_{3}\right) \delta 165.2,155.2,141.8,135.8,135.7,132.9,129.9,129.1,122.9,122.5,119.5,109.5,29.1$. HRMS (ESI+, m/z): [M+H] $]^{+}$calcd for $\mathrm{C}_{15} \mathrm{H}_{14} \mathrm{~N}_{3}{ }^{+}: 236.1182$, found: 236.1179 .

\section{Procedures and characterization data for triazinone products 3 via two-component reaction}

\section{General procedure for synthesis of triazinones}

In a $\mathrm{N}_{2}$-filled glovebox or on the benchtop, an oven-dried 2-5 mL Biotage microwave vial (\#351521) was charged with $\left[\mathrm{Cp}^{*} \mathrm{Rh}(\mathrm{MeCN})_{3}\right]\left(\mathrm{SbF}_{6}\right)_{2}(10 \mathrm{~mol} \%, 16.7 \mathrm{mg}, 0.0200 \mathrm{mmol})$, PivOH $(20.4 \mathrm{mg}, 0.200 \mathrm{mmol}$, 1.0 equiv), DCE (1.0 mL, $0.2 \mathrm{M})$, imine $(0.200 \mathrm{mmol}, 1.0$ equiv) and carbamylation reagent 2 (56.8 $\mathrm{mg}$, $0.300 \mathrm{mmol}, 1.5$ equiv). The vial was sealed with a teflon-lined septum and removed from the glovebox, or if set up on the benchtop, was flushed with nitrogen for $\sim 2 \mathrm{~min}$. The reaction mixture was heated for $20 \mathrm{~h}$ at $60{ }^{\circ} \mathrm{C}$ or $80^{\circ} \mathrm{C}$ in a pre-equilibrated oil bath. After cooling to rt, the reaction mixture was transferred to a vial with EtOAc and was concentrated in vacuo. The products were purified by the indicated chromatography techniques.

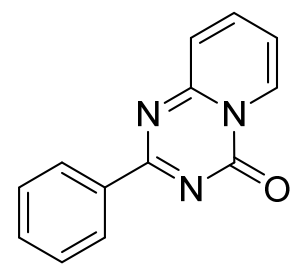

$3 a$

2-Phenyl-4H-pyrido[1,2-a][1,3,5]triazin-4-one (3a): The reaction was set up on the benchtop according to the General Procedure using imine 1a $(36.4 \mathrm{mg}, 0.200 \mathrm{mmol}, 1.0$ equiv), and the reaction mixture was stirred at $60{ }^{\circ} \mathrm{C}$. Purification by flash-column chromatography over silica gel (50\% EtOAc/hexanes) gave the desired product $3 \mathrm{a}$ as a white solid (34 $\mathrm{mg}, 76 \%$ yield). $\mathrm{mp} 189-190^{\circ} \mathrm{C}$. IR (neat) $1693,1624,1518$, 1394, 1343, 1126, 767, 701, 683, $534 \mathrm{~cm}^{-1} .{ }^{1} \mathrm{H} \mathrm{NMR}\left(500 \mathrm{MHz}, \mathrm{CDCl}_{3}\right) \delta 9.06$ (dd, $\left.J=7.0,1.6 \mathrm{~Hz}, 1 \mathrm{H}\right)$, $8.56(\mathrm{~d},, J=7.9 \mathrm{~Hz}, 2 \mathrm{H}), 8.01(\mathrm{td}, J=8.7,6.9 \mathrm{~Hz}, 1 \mathrm{H}), 7.68(\mathrm{~d}, J=8.9 \mathrm{~Hz}, 1 \mathrm{H}), 7.54(\mathrm{t}, J=7.0 \mathrm{~Hz}, 1 \mathrm{H})$, $7.48(\mathrm{t}, J=8.2 \mathrm{~Hz}, 2 \mathrm{H}), 7.30$ (td, $J=6.9,1.4 \mathrm{~Hz}, 1 \mathrm{H}) .{ }^{13} \mathrm{C} \mathrm{NMR}\left(101 \mathrm{MHz}, \mathrm{CDCl}_{3}\right) \delta$ 169.6, 155.6, 151.42, 141.7, 135.9, 132.8, 130.0, 129.6, 128.5, 125.6, 117.8. HRMS $(\mathrm{ESI}+, \mathrm{m} / \mathrm{z}):[\mathrm{M}+\mathrm{H}]+$ calcd for $\mathrm{C}_{13} \mathrm{H}_{10} \mathrm{~N}_{3} \mathrm{O}^{+}$: 224.0818, found: 224.0836 . 
<smiles>COc1ccc(-c2nc(=O)n3ccccc3n2)cc1</smiles>

$3 \mathbf{b}$

2-(4-Methoxyphenyl)-4H-pyrido[1,2-a][1,3,5]triazin-4-one (3b): The reaction was set up on the benchtop according to the General Procedure using imine $1 \mathrm{~b}(42.4 \mathrm{mg}, 0.200 \mathrm{mmol}, 1.0$ equiv), and the reaction mixture was stirred at $60{ }^{\circ} \mathrm{C}$. Purification by flash-column chromatography over silica gel $(70 \%$ EtOAc/hexanes) gave the desired product $3 \mathrm{~b}$ as an off-white solid $\left(43 \mathrm{mg}, 86 \%\right.$ yield). $\mathrm{mp} 230-231^{\circ} \mathrm{C}$. IR (neat) 1695, 1600, 1521, 1425, 1254, 1024, 844, 772, 713, $537 \mathrm{~cm}^{-1} .{ }^{1} \mathrm{H}$ NMR $\left(500 \mathrm{MHz}, \mathrm{CDCl}_{3}\right) \delta 9.05$ (dd, $J=7.0,1.6 \mathrm{~Hz}, 1 \mathrm{H}), 8.56(\mathrm{~d}, J=7.8 \mathrm{~Hz}, 2 \mathrm{H}), 7.98(\mathrm{t}, J=8.7 \mathrm{~Hz}, 1 \mathrm{H}), 7.65(\mathrm{~d}, J=8.8 \mathrm{~Hz}, 1 \mathrm{H}), 7.26(\mathrm{t}$, $J=7.8 \mathrm{~Hz}, 1 \mathrm{H}), 6.99(\mathrm{~d}, J=8.8 \mathrm{~Hz}, 2 \mathrm{H}), 3.90(\mathrm{~s}, 3 \mathrm{H}) .{ }^{13} \mathrm{C}$ NMR $\left(151 \mathrm{MHz}, \mathrm{CDCl}_{3}\right) \delta 169.2,163.7,155.8$, $151.4,141.4,131.8,130.0,128.5,125.4,117.2,113.9,55.6$. HRMS (ESI+, m/z): $[\mathrm{M}+\mathrm{H}]+$ calcd for $\mathrm{C}_{14} \mathrm{H}_{12} \mathrm{~N}_{3} \mathrm{O}_{2}{ }^{+}:$254.0924, found: 254.0945 .<smiles>O=c1nc(-c2ccc(-c3ccccc3)cc2)nc2ccccn12</smiles>

3c

2-([1,1'-Biphenyl]-4-yl)-4H-pyrido[1,2-a][1,3,5]triazin-4-one (3c): The reaction was set up on the benchtop according to the General Procedure using imine 1c (51.7 mg, $0.200 \mathrm{mmol}, 1.0$ equiv), and the reaction mixture was stirred at $60{ }^{\circ} \mathrm{C}$. Purification by flash-column chromatography over silica gel $(70 \%$ EtOAc/hexanes) gave the desired product $3 \mathrm{c}$ as a yellow solid $\left(40 \mathrm{mg}, 67 \%\right.$ yield). $\mathrm{mp} 223-224{ }^{\circ} \mathrm{C}$. IR (neat) 1701, 1630, 1510, 1445, 1301, 1119, 854, 767, 716, $533 \mathrm{~cm}^{-1} .{ }^{1} \mathrm{H} \mathrm{NMR}\left(500 \mathrm{MHz}, \mathrm{CDCl}_{3}\right) \delta 9.09$ (d, $J=6.9 \mathrm{~Hz}, 1 \mathrm{H}), 8.66(\mathrm{~d}, J=8.0 \mathrm{~Hz}, 2 \mathrm{H}), 8.02(\mathrm{t}, J=7.9 \mathrm{~Hz}, 1 \mathrm{H}), 7.75-7.66(\mathrm{~m}, 5 \mathrm{H}), 7.47(\mathrm{t}, J=7.6 \mathrm{~Hz}$, $2 \mathrm{H}), 7.39(\mathrm{t}, J=7.4 \mathrm{~Hz}, 1 \mathrm{H}), 7.32(\mathrm{t}, J=6.9 \mathrm{~Hz}, 1 \mathrm{H}) .{ }^{13} \mathrm{C}$ NMR $\left(151 \mathrm{MHz}, \mathrm{CDCl}_{3}\right) \delta$ 169.4, 155.6, 151.4, 145.4 141.6, 140.3, 134.8, 130.2, 130.1, 129.0, 128.1, 127.4, 127.2, 125.6, 117.7. HRMS (ESI+, m/z): $[\mathrm{M}+\mathrm{H}]+$ calcd for $\mathrm{C}_{19} \mathrm{H}_{14} \mathrm{~N}_{3} \mathrm{O}^{+}: 300.1131$, found: 300.1157 .<smiles>O=c1nc(-c2ccc(C(F)(F)F)cc2)nc2ccccn12</smiles> 
2-(4-(Trifluoromethyl)phenyl)-4H-pyrido[1,2-a][1,3,5]triazin-4-one (3d): The reaction was set up on the benchtop according to the General Procedure using imine $1 \mathbf{d}(50.0 \mathrm{mg}, 0.200 \mathrm{mmol}, 1.0$ equiv), and the reaction mixture was stirred at $60^{\circ} \mathrm{C}$. Purification by flash-column chromatography over silica gel $(70 \%$ EtOAc/hexanes) gave the desired product $3 \mathrm{~d}$ as a white solid ( $52 \mathrm{mg}, 89 \%$ yield). $\mathrm{mp} 170-172{ }^{\circ} \mathrm{C}$. IR (neat) 1726, 1517, 1457, 1426, 1315, 1171, 1109, 1061, 857, $785 \mathrm{~cm}^{-1} .{ }^{1} \mathrm{H}$ NMR $\left(400 \mathrm{MHz}, \mathrm{CDCl}_{3}\right) \delta 9.11$ (dd, $J$ $=6.9,1.6 \mathrm{~Hz}, 1 \mathrm{H}), 8.68(\mathrm{~d}, J=8.1 \mathrm{~Hz}, 2 \mathrm{H}), 8.08(\mathrm{t}, J=8.7 \mathrm{~Hz}, 1 \mathrm{H}), 7.74$ (overlapping d, $J=8.4 \mathrm{~Hz}, 3 \mathrm{H}$ ), 7.39 (t, $J=6.9 \mathrm{~Hz}, 1 \mathrm{H}) .{ }^{13} \mathrm{C} \mathrm{NMR}\left(151 \mathrm{MHz}, \mathrm{CDCl}_{3}\right) \delta 168.3,155.7,151.3,142.1,139.3,134.0(\mathrm{q}, J=32.4$ $\mathrm{Hz}), 130.2,129.9,125.7,125.5(\mathrm{q}, J=3.8 \mathrm{~Hz}), 123.2,118.4 .{ }^{19} \mathrm{~F} \mathrm{NMR}\left(376 \mathrm{MHz}, \mathrm{CDCl}_{3}\right) \delta-63.0 . \mathrm{HRMS}$ $(\mathrm{ESI}+, \mathrm{m} / \mathrm{z}):[\mathrm{M}+\mathrm{H}]+$ calcd for $\mathrm{C}_{14} \mathrm{H}_{9} \mathrm{~F}_{3} \mathrm{~N}_{3} \mathrm{O}^{+}: 292.0692$, found: 292.0713 .

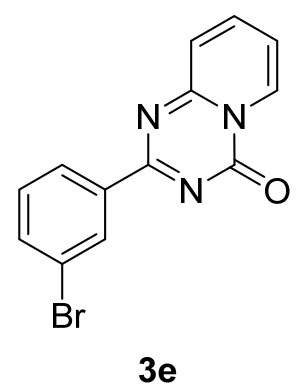

2-(3-Bromophenyl)-4H-pyrido[1,2-a][1,3,5]triazin-4-one (3e): The reaction was set up on the benchtop according to the General Procedure using imine 1e $(52.2 \mathrm{mg}, 0.200 \mathrm{mmol}, 1.0$ equiv), and the reaction mixture was stirred at $60{ }^{\circ} \mathrm{C}$. Purification by preparative thin-layer chromatography $(70 \% \mathrm{EtOAc/hexanes)}$ followed by a second preparative thin-layer chromatography $\left(2 \% \mathrm{MeOH} / \mathrm{CH}_{2} \mathrm{Cl}_{2}\right)$ purification to remove residual impurities gave the desired product $3 \mathrm{e}$ as a white solid $\left(43.5 \mathrm{mg}, 72 \%\right.$ yield). $\mathrm{mp} 227-230{ }^{\circ} \mathrm{C}$. IR (neat) $3108,1713,1627,1518,1457,1392,1332,792,714 \mathrm{~cm}^{-1} .{ }^{1} \mathrm{H}$ NMR $\left(600 \mathrm{MHz}, \mathrm{CDCl}_{3}\right) \delta 9.11(\mathrm{~d}, \mathrm{~J}=$ $6.8 \mathrm{~Hz}, 1 \mathrm{H}), 8.75(\mathrm{~s}, 1 \mathrm{H}), 8.52(\mathrm{~d}, J=8.5 \mathrm{~Hz}, 1 \mathrm{H}), 8.07(\mathrm{t}, J=7.8 \mathrm{~Hz}, 1 \mathrm{H}), 7.73(\mathrm{~d}, J=8.7 \mathrm{~Hz}, 1 \mathrm{H}), 7.68(\mathrm{~d}$, $J=7.4 \mathrm{~Hz}, 1 \mathrm{H}), 7.37(\mathrm{t}, J=7.5 \mathrm{~Hz}, 2 \mathrm{H}) .{ }^{13} \mathrm{C} \operatorname{NMR}\left(151 \mathrm{MHz}, \mathrm{CDCl}_{3}\right) \delta 168.4,155.7,151.3,142.0,138.0$, 135.6, 132.6, 130.2, 130.1, 128.2, 125.7, 122.9, 118.2. HRMS (ESI+, m/z): $[\mathrm{M}+\mathrm{H}]^{+}$calcd for $\mathrm{C}_{13} \mathrm{H}_{9} \mathrm{BrN}_{3} \mathrm{O}^{+}$: 301.9924, found: 301.9940 .<smiles>O=c1nc(-c2ccccc2Br)nc2ccccn12</smiles>

$3 \mathbf{f}$

2-(2-Bromophenyl)-4H-pyrido[1,2-a][1,3,5]triazin-4-one (3f): The reaction was set up on the benchtop according to the General Procedure using imine $1 \mathrm{f}(52.2 \mathrm{mg}, 0.200 \mathrm{mmol}, 1.0$ equiv), and the reaction mixture was stirred at $80{ }^{\circ} \mathrm{C}$. Purification by flash-column chromatography over silica gel $(80 \%$ EtOAc/hexanes) followed by preparative thin-layer chromatography ( $80 \%$ EtOAc/hexanes) gave the desired product $\mathbf{3 f}$ as a cream-colored solid (44.4 mg, $74 \%$ yield). $\mathrm{mp} 129-131^{\circ} \mathrm{C}$. IR (neat) 3122,3073 , 1706, 1621, 1519, 1444, 1388, 1336, 767, 731, $647 \mathrm{~cm}^{-1} .{ }^{1} \mathrm{H}$ NMR $\left(600 \mathrm{MHz}, \mathrm{CDCl}_{3}\right) \delta 9.15(\mathrm{~d}, J=6.9 \mathrm{~Hz}$, $1 \mathrm{H}), 8.16-8.07(\mathrm{~m}, 1 \mathrm{H}), 7.83(\mathrm{dd}, J=7.7,1.5 \mathrm{~Hz}, 1 \mathrm{H}), 7.76(\mathrm{~d}, J=8.7 \mathrm{~Hz}, 1 \mathrm{H}), 7.69(\mathrm{~d}, J=8.0 \mathrm{~Hz}, 1 \mathrm{H})$, 
$7.47-7.39(\mathrm{~m}, 2 \mathrm{H}), 7.31$ (td, $J=7.9,1.6 \mathrm{~Hz}, 1 \mathrm{H}) .{ }^{13} \mathrm{C}$ NMR $\left(151 \mathrm{MHz}, \mathrm{CDCl}_{3}\right) \delta$ 172.2, 155.4, 150.8, 142.3, $138.4,134.1,131.6,131.5,130.1,127.5,125.7,121.5,118.8$. HRMS $(E S I+, \mathrm{m} / \mathrm{z}):[\mathrm{M}+\mathrm{H}]^{+}$calcd for $\mathrm{C}_{13} \mathrm{H}_{9} \mathrm{BrN}_{3} \mathrm{O}^{+}: 301.9924$, found: 301.9939 .<smiles>O=c1nc(-c2ccccc2Cl)nc2ccccn12</smiles>

$3 g$

2-(2-Chlorophenyl)-4H-pyrido[1,2-a][1,3,5]triazin-4-one (3g): The reaction was set up on the benchtop according to the General Procedure using imine $1 \mathrm{~g}(43.3 \mathrm{mg}, 0.200 \mathrm{mmol}, 1.0$ equiv), and the reaction mixture was stirred at $60{ }^{\circ} \mathrm{C}$. Purification by flash-column chromatography over silica gel $(90 \%$ EtOAc/hexanes) gave the desired product $3 \mathrm{~g}$ as a yellow solid $\left(35 \mathrm{mg}, 68 \%\right.$ yield). $\mathrm{mp} 138-139{ }^{\circ} \mathrm{C}$. IR (neat) 1698, 1626, 1515, 1456, 1385, 1338, 1123, 1043, 767, 712, cm-1. ${ }^{1} \mathrm{H} \mathrm{NMR}\left(500 \mathrm{MHz}, \mathrm{CDCl}_{3}\right) \delta 9.14$ (dd, $J=6.9,1.7 \mathrm{~Hz}, 1 \mathrm{H}), 8.10(\mathrm{td}, J=8.7,6.9 \mathrm{~Hz}, 1 \mathrm{H}), 7.88(\mathrm{dd}, J=7.4,2.0 \mathrm{~Hz}, 1 \mathrm{H}), 7.75(\mathrm{~d}, J=8.8 \mathrm{~Hz}$, $1 \mathrm{H}), 7.48(\mathrm{dd}, J=7.7,1.6 \mathrm{~Hz}, 1 \mathrm{H}), 7.43(\mathrm{td}, J=6.6,1.4 \mathrm{~Hz}, 1 \mathrm{H}), 7.40-7.34(\mathrm{~m}, 2 \mathrm{H}) .{ }^{13} \mathrm{C}$ NMR $(151 \mathrm{MHz}$, $\left.\mathrm{CDCl}_{3}\right) \delta 171.3,155.3,150.8,142.3,136.5,132.8,131.6,131.4,130.8,129.9,126.8,125.6,118.8$. HRMS (ESI+, m/z): $[\mathrm{M}+\mathrm{H}]+$ calcd for $\mathrm{C}_{13} \mathrm{H}_{9} \mathrm{CIN}_{3} \mathrm{O}^{+}: 258.0429$, found: 258.0447 .<smiles>O=c1nc(-c2c(Cl)cccc2Cl)nc2ccccn12</smiles>

$3 \mathrm{~h}$

2-(2,6-Dichlorophenyl)-4H-pyrido[1,2-a][1,3,5]triazin-4-one (3h): The reaction was set up inside an $\mathrm{N}_{2}-$ filled glovebox according to the General Procedure using imine $1 \mathrm{~h}$ (50.2 $\mathrm{mg}, 0.200 \mathrm{mmol}, 1.0$ equiv), and the reaction mixture was stirred at $80^{\circ} \mathrm{C}$. Purification by flash-column chromatography over silica gel $(70 \%$ EtOAc/hexanes) gave the desired product $3 \mathrm{~h}$ as a white solid ( $26 \mathrm{mg}, 44 \%$ yield). $\mathrm{mp} 216-218{ }^{\circ} \mathrm{C}$. IR (neat) 1708, 1630, 1509, 1443, 1394, 1306, 1187, 941, 757, $711 \mathrm{~cm}^{-1} .{ }^{1} \mathrm{H} \mathrm{NMR}\left(600 \mathrm{MHz}, \mathrm{CDCl}_{3}\right) \delta 9.17(\mathrm{dd}, J=$ 6.9, $1.7 \mathrm{~Hz}, 1 \mathrm{H}), 8.16$ (ddd, $J=8.7,6.9,1.7 \mathrm{~Hz}, 1 \mathrm{H}), 7.77(\mathrm{~d}, J=8.9 \mathrm{~Hz}, 1 \mathrm{H}), 7.51(\mathrm{td}, J=7.0,1.3 \mathrm{~Hz}, 1 \mathrm{H})$, $7.38(\mathrm{~d}, J=8.1 \mathrm{~Hz}, 2 \mathrm{H}), 7.29(\mathrm{dd}, J=8.7,7.5 \mathrm{~Hz}, 1 \mathrm{H}) .{ }^{13} \mathrm{C} \mathrm{NMR}\left(151 \mathrm{MHz}, \mathrm{CDCl}_{3}\right) \delta 170.4,155.8,150.6$, $142.7,136.7,133.0,130.5,130.2,128.2,125.7,119.3$. HRMS $(E S I+, m / z):[M+H]+$ calcd for $\mathrm{C}_{13} \mathrm{H}_{8} \mathrm{Cl}_{2} \mathrm{~N}_{3} \mathrm{O}^{+}$: 292.0039, found: 292.0062 . 
<smiles>O=c1nc(-c2cccnc2)nc2ccccn12</smiles>

$3 \mathbf{i}$

2-(Pyridin-3-yl)-4H-pyrido[1,2-a][1,3,5]triazin-4-one (3i): The reaction was set up inside an $\mathrm{N}_{2}$-filled glovebox according to the General Procedure using imine $1 \mathrm{i}$ (36.6 mg, $0.200 \mathrm{mmol}, 1.0$ equiv), and the reaction mixture was stirred at $80{ }^{\circ} \mathrm{C}$. Purification by flash-column chromatography over silica gel $(95 \%$ EtOAc/hexanes) gave the desired product $3 \mathbf{i}$ as a white solid $\left(23 \mathrm{mg}, 51 \%\right.$ yield). $\mathrm{mp} 152-153^{\circ} \mathrm{C}$. IR (neat) 1701, 1518, 1439, 1391, 1307, 1023, 869, 769, 699, $535 \mathrm{~cm}^{-1} .{ }^{1} \mathrm{H} \mathrm{NMR}\left(500 \mathrm{MHz}, \mathrm{CDCl}_{3}\right) \delta 9.75(\mathrm{~s}, 1 \mathrm{H})$, $9.13(\mathrm{~d}, J=7.0 \mathrm{~Hz}, 1 \mathrm{H}), 8.83(\mathrm{dt}, J=8.0,2.0 \mathrm{~Hz}, 1 \mathrm{H}), 8.78(\mathrm{~d}, J=4.9 \mathrm{~Hz}, 1 \mathrm{H}), 8.09$ (td, $J=8.7,6.9 \mathrm{~Hz}$, $1 \mathrm{H}), 7.76(\mathrm{~d}, J=8.8 \mathrm{~Hz}, 1 \mathrm{H}), 7.44(\mathrm{dd}, J=8.0,4.8 \mathrm{~Hz}, 1 \mathrm{H}), 7.40(\mathrm{td}, J=6.9,1.3 \mathrm{~Hz}, 1 \mathrm{H}) .{ }^{13} \mathrm{C}$ NMR $(151$ $\left.\mathrm{MHz}, \mathrm{CDCl}_{3}\right) \delta 168.3,155.7,153.1,151.2,151.1,142.2,136.9,131.6,130.2,125.7,123.5,118.4 . \mathrm{HRMS}$ $(\mathrm{ESI}+, \mathrm{m} / \mathrm{z}):[\mathrm{M}+\mathrm{H}]+$ calcd for $\mathrm{C}_{12} \mathrm{H}_{9} \mathrm{~N}_{4} \mathrm{O}^{+}: 225.0771$, found: 225.0788 .<smiles>O=c1nc(-c2ccc(Cl)nc2)nc2ccccn12</smiles>

3j

2-(6-Chloropyridin-3-yl)-4H-pyrido[1,2-a][1,3,5]triazin-4-one (3j): The reaction was set up inside an $\mathrm{N}_{2}$ filled glovebox according to the General Procedure using imine $1 \mathrm{j}$ (43.5 mg, $0.200 \mathrm{mmol}, 1.0$ equiv), and $\mathrm{NaOAc}\left(16.4 \mathrm{mg}, 0.200 \mathrm{mmol}\right.$. 1.0 equiv), and the reaction mixture was stirred at $80^{\circ} \mathrm{C}$. Purification by flash-column chromatography over silica gel (30\% EtOAc/hexanes) gave the desired product $\mathbf{3} \mathbf{j}$ as a white solid (35 mg, 67\% yield). mp 251-252 ${ }^{\circ} \mathrm{C}$. IR (neat) 1708, 1580, 1514, 1411, 1349, 1104, 1016, 782, 719, $541 \mathrm{~cm}^{-1} .{ }^{1} \mathrm{H}$ NMR $\left(600 \mathrm{MHz}, \mathrm{CDCl}_{3}\right) \delta 9.51(\mathrm{dd}, J=2.3,0.7 \mathrm{~Hz}, 1 \mathrm{H}), 9.12$ (ddd, $J=6.8,1.6,0.7 \mathrm{~Hz}, 1 \mathrm{H}$ ), $8.77(\mathrm{dd}, J=8.4,2.4 \mathrm{~Hz}, 1 \mathrm{H}), 8.11$ (ddd, $J=8.7,6.9,1.7 \mathrm{~Hz}, 1 \mathrm{H}), 7.75(\mathrm{dt}, J=8.8,1.1 \mathrm{~Hz}, 1 \mathrm{H}$ ), 7.46 (dd, $J=8.4,0.7 \mathrm{~Hz}, 1 \mathrm{H}), 7.42(\mathrm{td}, J=6.9,1.3 \mathrm{~Hz}, 1 \mathrm{H}) .{ }^{13} \mathrm{C}$ NMR $\left(151 \mathrm{MHz}, \mathrm{CDCl}_{3}\right) \delta 167.3,155.7,155.4,151.4$, 151.0, 142.4, 139.5, 130.7, 130.3, 125.7, 124.2, 118.6. HRMS (ESI+, m/z): $[\mathrm{M}+\mathrm{H}]+$ calcd for $\mathrm{C}_{12} \mathrm{H}_{8} \mathrm{CIN}_{4} \mathrm{O}^{+}$: 259.0381, found: 259.0402 .<smiles>Cn1cc(-c2nc(=O)n3ccccc3n2)cn1</smiles>

3k 
2-(1-Methyl-1H-pyrazol-4-yl)-4H-pyrido[1,2-a][1,3,5]triazin-4-one (3k): The reaction was set up inside a $\mathrm{N}_{2}$-filled glovebox according to the General Procedure using imine $1 \mathrm{k}$ (37.2 $\mathrm{mg}, 0.200 \mathrm{mmol}, 1.0$ equiv), and the reaction mixture was stirred at $80{ }^{\circ} \mathrm{C}$. Purification by preparative thin-layer chromatography $(5 \%$ $\mathrm{MeOH} / \mathrm{EtOAc}$ ) gave the desired product $\mathbf{3 k}$ as a cream-colored solid $\left(39.8 \mathrm{mg}, 88 \%\right.$ yield). $\mathrm{mp} 158{ }^{\circ} \mathrm{C}$ (decomp). IR (neat) 3119, 1785, 1696, 1556, 1519, 1421, 1358, 1310, 771, $613 \mathrm{~cm}^{-1}$. ${ }^{1} \mathrm{H}$ NMR $(400 \mathrm{MHz}$, $\left.50 \% \mathrm{CDCl}_{3}: \mathrm{MeOD}\right) \delta 9.01(\mathrm{~d}, J=6.9 \mathrm{~Hz}, 1 \mathrm{H}), 8.32(\mathrm{~s}, 1 \mathrm{H}), 8.22(\mathrm{~s}, 1 \mathrm{H}), 8.20-8.13(\mathrm{~m}, 1 \mathrm{H}), 7.67(\mathrm{~d}, J=$ $8.8 \mathrm{~Hz}, 1 \mathrm{H}), 7.45(\mathrm{td}, J=6.9,1.0 \mathrm{~Hz}, 1 \mathrm{H}), 3.97(\mathrm{~s}, 3 \mathrm{H}) .{ }^{13} \mathrm{C} \mathrm{NMR}\left(151 \mathrm{MHz}, 50 \% \mathrm{CDCl}_{3}: \mathrm{MeOD}\right) \delta 166.1$, 156.2, 151.9, 143.4, 141.7, 134.5, 130.4, 125.2, 121.9, 118.7, 39.4. HRMS (ESI+, m/z): $[\mathrm{M}+\mathrm{H}]^{+}$calcd for $\mathrm{C}_{11} \mathrm{H}_{10} \mathrm{~N}_{5} \mathrm{O}^{+}: 228.0880$, found: 228.0891 .

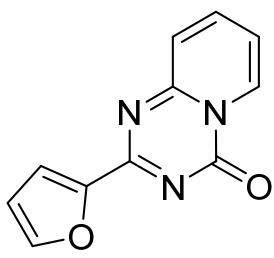

31

2-(Furan-2-yl)-4H-pyrido[1,2-a][1,3,5]triazin-4-one (3l): ${ }^{11}$ The reaction was set up on the benchtop according to the General Procedure using imine 11 (34.4 $\mathrm{mg}, 0.200 \mathrm{mmol}, 1.0$ equiv), and the reaction mixture was stirred at $60{ }^{\circ} \mathrm{C}$. Purification by flash-column chromatography over silica gel $(90 \%$ EtOAc/hexanes) followed by preparative thin-layer chromatography (95\% EtOAc/hexanes) gave the desired product $3 \mathbf{I}$ as a cream-colored solid $(31.3 \mathrm{mg}, 73 \%$ yield). Spectroscopic data are in agreement with reported values. ${ }^{11}{ }^{1} \mathrm{H}$ NMR $\left(600 \mathrm{MHz}, \mathrm{CDCl}_{3}\right) \delta 9.08(\mathrm{dd}, J=6.9,0.9 \mathrm{~Hz}, 1 \mathrm{H}), 8.10-7.99(\mathrm{~m}, 1 \mathrm{H})$, $7.74(\mathrm{~d}, J=8.8 \mathrm{~Hz}, 1 \mathrm{H}), 7.72(\mathrm{dd}, J=1.6,0.8 \mathrm{~Hz}, 1 \mathrm{H}), 7.62(\mathrm{dd}, J=3.5,0.7 \mathrm{~Hz}, 1 \mathrm{H}), 7.33(\mathrm{td}, J=6.9,1.3$ $\mathrm{Hz}, 1 \mathrm{H}), 6.62(\mathrm{dd}, J=3.5,1.7 \mathrm{~Hz}, 1 \mathrm{H}) .{ }^{13} \mathrm{C} \mathrm{NMR}\left(151 \mathrm{MHz}, \mathrm{CDCl}_{3}\right) \delta 161.6,156.0,150.8,150.7,147.3$, $142.1,130.4,125.4,118.6,117.9,113.1$.

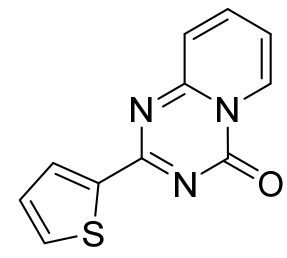

$3 m$

2-(Thiophen-2-yl)-4H-pyrido[1,2-a][1,3,5]triazin-4-one $(3 \mathrm{~m}):^{11}$ The reaction was set up on the benchtop according to the General Procedure using imine $1 \mathrm{~m}(37.6 \mathrm{mg}, 0.200 \mathrm{mmol}, 1.0$ equiv), and the reaction mixture was stirred at $60{ }^{\circ} \mathrm{C}$. Purification by flash-column chromatography over silica gel $(5 \%$ $\left.\mathrm{MeOH} / \mathrm{CH}_{2} \mathrm{Cl}_{2}\right)$ followed by preparative thin-layer chromatography $(70 \%$ EtOAc/hexanes) gave the desired product $3 \mathrm{~m}$ as a cream-colored solid $(38.0 \mathrm{mg}, 83 \%$ yield). Spectroscopic data are in agreement with reported values. ${ }^{11}{ }^{1} \mathrm{H}$ NMR $\left(600 \mathrm{MHz}, \mathrm{CDCl}_{3}\right) \delta 9.06(\mathrm{~d}, J=7.7 \mathrm{~Hz}, 1 \mathrm{H}), 8.22(\mathrm{dd}, J=3.7,1.1 \mathrm{~Hz}, 1 \mathrm{H}), 8.03$ - $7.97(\mathrm{~m}, 1 \mathrm{H}), 7.68-7.60(\mathrm{~m}, 2 \mathrm{H}), 7.28(\mathrm{td}, J=6.9,1.2 \mathrm{~Hz}, 1 \mathrm{H}), 7.19(\mathrm{dd}, J=4.9,3.8 \mathrm{~Hz}, 1 \mathrm{H}) .{ }^{13} \mathrm{C}$ NMR $\left(151 \mathrm{MHz}, \mathrm{CDCl}_{3}\right) \delta 165.8,155.7,150.9,141.8,141.7,133.6,132.9,130.3,128.8,125.2,117.5$. 
<smiles>COC(=O)c1ccc(-c2nc(=O)n3ccccc3n2)cc1</smiles>

$3 n$

Methyl 4-(4-oxo-4H-pyrido[1,2-a][1,3,5]triazin-2-yl)benzoate (3n): The reaction was set up inside an $\mathrm{N}_{2}$ filled glovebox according to the General Procedure using imine $1 \mathrm{n}(48.1 \mathrm{mg}, 0.200 \mathrm{mmol}, 1.0$ equiv), and the reaction mixture was stirred at $60^{\circ} \mathrm{C}$. Isolation by vacuum filtration followed by trituration with EtOAc gave the desired product $3 \mathrm{n}$ as a white solid $\left(41 \mathrm{mg}, 73 \%\right.$ yield). $\mathrm{mp} 246-248{ }^{\circ} \mathrm{C}$. IR (neat) 1711,1629 , 1519, 1423, 1277, 1106, 868, 772, 712, $539 \mathrm{~cm}^{-1} .{ }^{1} \mathrm{H}$ NMR $\left(600 \mathrm{MHz}, \mathrm{CDCl}_{3}\right) \delta 9.12$ (d, J = $\left.6.0 \mathrm{~Hz}, 1 \mathrm{H}\right)$, $8.65(\mathrm{~d}, J=7.2 \mathrm{~Hz}, 2 \mathrm{H}), 8.15(\mathrm{~d}, J=8.5 \mathrm{~Hz}, 2 \mathrm{H}), 8.08(\mathrm{td}, J=8.7,6.9 \mathrm{~Hz}, 1 \mathrm{H}), 7.75(\mathrm{~d}, J=8.8 \mathrm{~Hz}, 1 \mathrm{H})$, $7.38(\mathrm{td}, J=6.9,1.4 \mathrm{~Hz}, 1 \mathrm{H}), 3.96(\mathrm{~s}, 3 \mathrm{H}) .{ }^{13} \mathrm{C} \mathrm{NMR}\left(151 \mathrm{MHz}, \mathrm{CDCl}_{3}\right) \delta 168.8,166.8,155.7,151.3,142.0$, 140.0, 133.6, 130.2, 129.7, 129.6, 125.8, 118.3, 52.5. HRMS (ESI+, m/z): $[\mathrm{M}+\mathrm{H}]+$ calcd for $\mathrm{C}_{15} \mathrm{H}_{12} \mathrm{~N}_{3} \mathrm{O}_{3}{ }^{+}$: 282.0873, found: 282.0896 .<smiles>O=c1nc(-c2ccccc2O)nc2ccccn12</smiles>

30

2-(2-Hydroxyphenyl)-4H-pyrido[1,2-a][1,3,5]triazin-4-one (3o): The reaction was set up inside an $\mathrm{N}_{2}$ filled glovebox according to the General Procedure using imine 10 (39.6 mg, $0.200 \mathrm{mmol}, 1.0$ equiv), and the reaction mixture was stirred at $60^{\circ} \mathrm{C}$. Purification by flash-column chromatography over silica gel $(10 \%$ EtOAc/hexanes) gave the desired product 30 as a white solid ( $25 \mathrm{mg}, 52 \%$ yield). $\mathrm{mp} 170-171^{\circ} \mathrm{C}$. IR (neat) $3058,1732,1643,1583,1455,1374,1206,1139,959,845,729 \mathrm{~cm}^{-1} .{ }^{1} \mathrm{H}$ NMR $\left(400 \mathrm{MHz}, \mathrm{CDCl}_{3}\right) \delta 8.42$ (d, $J=5.1 \mathrm{~Hz}, 1 \mathrm{H}), 8.32$ (dd, $J=7.9,1.7 \mathrm{~Hz}, 1 \mathrm{H}), 7.78(\mathrm{td}, J=7.6,2.0 \mathrm{~Hz}, 1 \mathrm{H}), 7.60$ (td, $J=8.7,7.3 \mathrm{~Hz}$, $1 \mathrm{H}), 7.43$ (d, $J=8.1 \mathrm{~Hz}, 1 \mathrm{H}), 7.34(\mathrm{td}, J=8.2,7.4 \mathrm{~Hz}, 1 \mathrm{H}), 7.23$ (d, $J=8.3 \mathrm{~Hz}, 1 \mathrm{H}$ ), 7.13 (td, $J=7.4,5.0$ $\mathrm{Hz}, 1 \mathrm{H}) .{ }^{13} \mathrm{C} \mathrm{NMR}\left(151 \mathrm{MHz}, \mathrm{CDCl}_{3}\right) \delta 160.3,153.2,147.1,146.5,146.3,138.7,134.3,126.6,125.3,124.0$, 120.5, 116.8, 116.0. HRMS (ESI+, m/z): $[\mathrm{M}+\mathrm{H}]+$ calcd for $\mathrm{C}_{13} \mathrm{H}_{10} \mathrm{~N}_{3} \mathrm{O}_{2}{ }^{+}: 240.0768$, found: 240.0785 .<smiles>CC(C)Nc1ccc(-c2nc(=O)n3ccccc3n2)cc1</smiles>

$3 p$ 
$N$-(4-(4-Oxo-4H-pyrido[1,2-a][1,3,5]triazin-2-yl)phenyl)acetamide (3p): The reaction was set up inside a $\mathrm{N}_{2}$-filled glovebox according to the General Procedure using imine $1 \mathrm{p}$ ( $47.9 \mathrm{mg}, 0.200 \mathrm{mmol}, 1.0$ equiv), and the reaction mixture was stirred at $60{ }^{\circ} \mathrm{C}$. The product was filtered and rinsed with $\mathrm{CH}_{2} \mathrm{Cl}_{2}$ followed by $\mathrm{MeOH}$ to yield the desired product $3 \mathrm{p}$ as a white solid ( $34.5 \mathrm{mg}, 62 \%$ yield). $\mathrm{mp} 272{ }^{\circ} \mathrm{C}$ (decomp). IR (neat) 3256, 3117, 1682, 1595, 1514, 1420, 1321, 1266, 852, $775 \mathrm{~cm}^{-1} .{ }^{1} \mathrm{H}$ NMR $\left.\left(600 \mathrm{MHz},\left(\mathrm{CD}_{3}\right)_{2} \mathrm{SO}\right)\right) \delta 10.27$ (s, $1 \mathrm{H}), 8.97(\mathrm{~d}, J=6.8 \mathrm{~Hz}, 1 \mathrm{H}), 8.39(\mathrm{~d}, J=8.7 \mathrm{~Hz}, 2 \mathrm{H}), 8.31-8.20(\mathrm{~m}, 1 \mathrm{H}), 7.75(\mathrm{~d}, J=8.9 \mathrm{~Hz}, 2 \mathrm{H}), 7.73$ $(\mathrm{d}, J=8.9 \mathrm{~Hz}, 1 \mathrm{H}), 7.51(\mathrm{t}, J=7.2 \mathrm{~Hz}, 1 \mathrm{H}), 2.10(\mathrm{~s}, 3 \mathrm{H}) .{ }^{13} \mathrm{C} \mathrm{NMR}\left(151 \mathrm{MHz},\left(\mathrm{CD}_{3}\right)_{2} \mathrm{SO}\right) \delta$ 168.8, 167.1, $155.1,150.6,143.2,143.0,130.3,129.9,129.8,124.8,118.5,118.4,24.2$. HRMS (ESI+, m/z): $[\mathrm{M}+\mathrm{H}]^{+}$calcd for $\mathrm{C}_{15} \mathrm{H}_{13} \mathrm{~N}_{4} \mathrm{O}_{2}{ }^{+}: 281.1033$, found: 281.1057 .

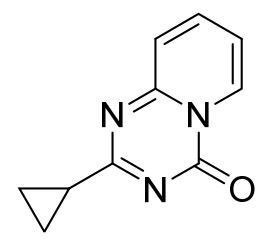

$3 q$

2-Cyclopropyl-4H-pyrido[1,2-a][1,3,5]triazin-4-one (3q): The reaction was set up inside a $\mathrm{N}_{2}$-filled glovebox according to the General Procedure using imine 1q $(29.2 \mathrm{mg}, 0.200 \mathrm{mmol}, 1.0$ equiv), and the reaction mixture was stirred at $80{ }^{\circ} \mathrm{C}$. Purification by preparative thin-layer chromatography $(90 \%$ EtOAc/hexanes) gave the desired product $3 q$ as a cream-colored solid (28.2 mg, 75\% yield). mp 167-168 ${ }^{\circ} \mathrm{C}$. IR (neat) 3106, 3013, 1707, 1628, 1518, 1464, 1441, 1398, 976, $783 \mathrm{~cm}^{-1} .{ }^{1} \mathrm{H} \mathrm{NMR}\left(600 \mathrm{MHz}, \mathrm{CDCl}_{3}\right)$ $\delta 8.98(\mathrm{~d}, J=7.2 \mathrm{~Hz}, 1 \mathrm{H}), 8.00-7.91(\mathrm{~m}, 1 \mathrm{H}), 7.51(\mathrm{~d}, J=8.8 \mathrm{~Hz}, 1 \mathrm{H}), 7.24(\mathrm{td}, J=6.9,1.0 \mathrm{~Hz}, 1 \mathrm{H}), 2.13$ $-2.03(\mathrm{~m}, 1 \mathrm{H}), 1.37-1.33(\mathrm{~m}, 2 \mathrm{H}), 1.14-1.09(\mathrm{~m}, 2 \mathrm{H}) .{ }^{13} \mathrm{C}$ NMR $\left(151 \mathrm{MHz}, \mathrm{CDCl}_{3}\right) \delta 180.0,155.0,150.7$, 141.6, 129.9, 124.6, 117.1, 19.0, 12.1. HRMS (ESI+, m/z): $[\mathrm{M}+\mathrm{H}]^{+}$calcd for $\mathrm{C}_{10} \mathrm{H}_{10} \mathrm{~N}_{3} \mathrm{O}^{+}: 188.0818$, found: 188.0832 .

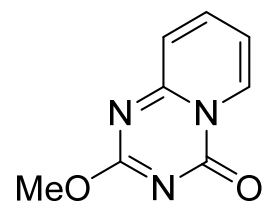

$3 r$

2-Methoxy-4H-pyrido[1,2-a][1,3,5]triazin-4-one (3r): The reaction was set up inside a $\mathrm{N}_{2}$-filled glovebox according to the General Procedure with minor modifications. In place of DCE, the reaction was run in TFE $(1.0 \mathrm{~mL})$ using imine $1 \mathrm{r}\left(27.2 \mathrm{mg}, 0.200 \mathrm{mmol}, 1.0\right.$ equiv), and the reaction mixture was stirred at $80^{\circ} \mathrm{C}$. Purification by preparative thin-layer chromatography $(5 \% \mathrm{MeOH} / \mathrm{EtOAc})$ gave the desired product $3 \mathrm{r}$ as a white solid (13.9 $\mathrm{mg}, 39 \%$ yield). $\mathrm{mp} 164-167^{\circ} \mathrm{C}$. IR (neat) 3116, 1709, 1630, 1571, 1519, 1447, 1339, $782,714 \mathrm{~cm}^{-1} .{ }^{1} \mathrm{H}$ NMR $\left(600 \mathrm{MHz}, \mathrm{CDCl}_{3}\right) \delta 8.92(\mathrm{dd}, J=7.0,0.9 \mathrm{~Hz}, 1 \mathrm{H}), 7.97-7.85(\mathrm{~m}, 1 \mathrm{H}), 7.45(\mathrm{~d}, J$ $=8.8 \mathrm{~Hz}, 1 \mathrm{H}), 7.16(\mathrm{td}, J=6.9,1.2 \mathrm{~Hz}, 1 \mathrm{H}), 4.07(\mathrm{~s}, 3 \mathrm{H}) .{ }^{13} \mathrm{C} \mathrm{NMR}\left(151 \mathrm{MHz}, \mathrm{CDCl}_{3}\right) \delta 168.3,156.6,152.1$, 141.8, 130.1, 124.2, 116.0, 55.7. HRMS (ESI+, $\mathrm{m} / \mathrm{z}):[\mathrm{M}+\mathrm{H}]^{+}$calcd for $\mathrm{C}_{8} \mathrm{H}_{8} \mathrm{~N}_{3} \mathrm{O}_{2}{ }^{+}:$178.0611, found: 178.0623. 
<smiles>Cc1cccn2c(=O)nc(-c3ccccc3)nc12</smiles>

3s

9-Methyl-2-phenyl-4H-pyrido[1,2-a][1,3,5]triazin-4-one (3s): The reaction was set up on the benchtop according to the General Procedure using imine $1 \mathrm{~s}(39.2 \mathrm{mg}, 0.200 \mathrm{mmol}, 1.0$ equiv), and the reaction mixture was stirred at $60{ }^{\circ} \mathrm{C}$. Purification by flash-column chromatography over silica gel $(60 \%$ EtOAc/hexanes) gave the desired product 3 s as a yellow solid (35 mg, $73 \%$ yield). $\mathrm{mp} 170-172{ }^{\circ} \mathrm{C}$. IR (neat) 1693, 1625, 1509, 1425, 1344, 1161, 1081, 766, 707, $684 \mathrm{~cm}^{-1} .{ }^{1} \mathrm{H}$ NMR $\left(400 \mathrm{MHz}, \mathrm{CDCl}_{3}\right) \delta 8.95$ $(\mathrm{d}, J=7.0 \mathrm{~Hz}, 1 \mathrm{H}), 8.60(\mathrm{~d}, J=7.6 \mathrm{~Hz}, 2 \mathrm{H}), 7.83(\mathrm{~d}, J=7.1 \mathrm{~Hz}, 1 \mathrm{H}), 7.55(\mathrm{t}, J=7.2 \mathrm{~Hz}, 1 \mathrm{H}), 7.48(\mathrm{t}, J=$ $8.2 \mathrm{~Hz}, 2 \mathrm{H}), 7.19(\mathrm{t}, J=7.0 \mathrm{~Hz}, 1 \mathrm{H}), 2.67(\mathrm{~s}, 3 \mathrm{H}) .{ }^{13} \mathrm{C} \mathrm{NMR}\left(126 \mathrm{MHz}, \mathrm{CDCl}_{3}\right) \delta 168.5,154.9,152.0,140.2$, 136.2, 134.8, 132.6, 129.6, 128.4, 127.7, 117.1, 17.5. HRMS $(\mathrm{ESI}+, \mathrm{m} / \mathrm{z}):[\mathrm{M}+\mathrm{H}]+$ calcd for $\mathrm{C}_{14} \mathrm{H}_{12} \mathrm{~N}_{3} \mathrm{O}^{+}$: 238.0975, found: 238.0995 .<smiles>Cc1ccn2c(=O)nc(-c3ccccc3)nc2c1</smiles>

3t

8-Methyl-2-phenyl-4H-pyrido[1,2-a][1,3,5]triazin-4-one (3t): The reaction was set up on the benchtop according to the General Procedure using imine 1t $(39.2 \mathrm{mg}, 0.200 \mathrm{mmol}, 1.0$ equiv), and the reaction mixture was stirred at $60{ }^{\circ} \mathrm{C}$. Purification by flash-column chromatography over silica gel $(60 \%$ EtOAc/hexanes) gave the desired product $3 \mathrm{t}$ as a white solid $\left(33 \mathrm{mg}, 69 \%\right.$ yield). $\mathrm{mp} 234-235^{\circ} \mathrm{C}$. IR (neat) 1693, 1639, 1505, 1408, 1342, 1147, 864, 775, 706, $687 \mathrm{~cm}^{-1} .{ }^{1} \mathrm{H}$ NMR $\left(600 \mathrm{MHz}, \mathrm{CDCl}_{3}\right) \delta 8.95(\mathrm{~d}, J=$ $7.0 \mathrm{~Hz}, 1 \mathrm{H}), 8.55(\mathrm{~d}, J=7.7 \mathrm{~Hz}, 2 \mathrm{H}), 7.55(\mathrm{t}, J=7.3 \mathrm{~Hz}, 1 \mathrm{H}), 7.51-7.44(\mathrm{~m}, 3 \mathrm{H}), 7.12(\mathrm{~d}, J=7.1 \mathrm{~Hz}, 1 \mathrm{H})$, $2.55(\mathrm{~s}, 3 \mathrm{H}) .{ }^{13} \mathrm{C}$ NMR $\left(151 \mathrm{MHz}, \mathrm{CDCl}_{3}\right) \delta 169.7,155.3,154.7,151.6,136.1,132.6,129.6,129.3,128.5$, 123.9, 120.2, 22.1. HRMS (ESI+, m/z): [M+H] + calcd for $\mathrm{C}_{14} \mathrm{H}_{12} \mathrm{~N}_{3} \mathrm{O}^{+}: 238.0975$, found: 238.0995 .<smiles>Cc1ccc2nc(-c3ccccc3)nc(=O)n2c1</smiles>

3u

7-Methyl-2-phenyl-4H-pyrido[1,2-a][1,3,5]triazin-4-one (3u): The reaction was set up on the benchtop according to the General Procedure using imine $1 \mathrm{u}(39.2 \mathrm{mg}, 0.200 \mathrm{mmol}, 1.0$ equiv), and the reaction mixture was stirred at $60{ }^{\circ} \mathrm{C}$. Purification by flash-column chromatography over silica gel $(60 \%$ EtOAc/hexanes) gave the desired product $3 \mathbf{u}$ as a white solid ( $31 \mathrm{mg}, 65 \%$ yield). $\mathrm{mp} 209-210{ }^{\circ} \mathrm{C}$. IR (neat) 
1697, 1642, 1524, 1409, 1376, 1219, 1044, 831, 782, 706, $539 \mathrm{~cm}^{-1} .{ }^{1} \mathrm{H}$ NMR $\left(600 \mathrm{MHz}, \mathrm{CDCl}_{3}\right) \delta 8.90(\mathrm{~s}$, $1 \mathrm{H}), 8.56$ (d, $J=7.5 \mathrm{~Hz}, 2 \mathrm{H}), 7.87(\mathrm{~d}, J=8.9 \mathrm{~Hz}, 1 \mathrm{H}), 7.63(\mathrm{~d}, J=8.9 \mathrm{~Hz}, 1 \mathrm{H}), 7.55$ (t, $J=7.4 \mathrm{~Hz}, 1 \mathrm{H}), 7.49$ $(\mathrm{t}, J=7.6 \mathrm{~Hz}, 2 \mathrm{H}), 2.47(\mathrm{~s}, 3 \mathrm{H}) .{ }^{13} \mathrm{C}$ NMR $\left(151 \mathrm{MHz}, \mathrm{CDCl}_{3}\right) \delta 169.0,154.2,151.5,144.3,136.0,132.6$, 129.5, 128.54, 128.58, 127.8, 125.0, 18.5. HRMS $(\mathrm{ESI}+, \mathrm{m} / \mathrm{z}):[\mathrm{M}+\mathrm{H}]+$ calcd for $\mathrm{C}_{14} \mathrm{H}_{12} \mathrm{~N}_{3} \mathrm{O}^{+}: 238.0975$, found: 238.0996.<smiles>Cc1cccc2nc(-c3ccccc3)nc(=O)n12</smiles>

\section{3v}

6-Methyl-2-phenyl-4H-pyrido[1,2-a][1,3,5]triazin-4-one (3v): The reaction was set up inside an $\mathrm{N}_{2}$-filled glovebox according to the General Procedure using imine $1 \mathrm{v}$ (50.2 mg, $0.200 \mathrm{mmol}, 1.0$ equiv) and NaOAc (16.4 mg, $0.200 \mathrm{mmol}, 1.0$ equiv), and the reaction mixture was stirred at $80^{\circ} \mathrm{C}$. Purification by flash-column chromatography over silica gel ( $30 \%$ EtOAc/hexanes) gave the desired product $3 \mathbf{v}$ as a white solid (16 mg, $34 \%$ yield). mp 177-178 ${ }^{\circ} \mathrm{C}$. IR (neat) 1694, 1626, 1515, 1422, 1264, 1025, 810, 784, 699, $661 \mathrm{~cm}^{-1} .{ }^{1} \mathrm{H}$ NMR (400 MHz, $\left.\mathrm{CDCl}_{3}\right) \delta 8.51(\mathrm{~d}, J=7.6 \mathrm{~Hz}, 2 \mathrm{H}), 7.74$ (dd, $\left.J=8.8,7.1 \mathrm{~Hz}, 1 \mathrm{H}\right), 7.54$ (t, $\left.J=7.2 \mathrm{~Hz}, 1 \mathrm{H}\right)$, $7.50-7.44(\mathrm{~m}, 3 \mathrm{H}), 6.92(\mathrm{~d}, J=7.1 \mathrm{~Hz}, 1 \mathrm{H}), 3.05(\mathrm{~s}, 3 \mathrm{H}) .{ }^{13} \mathrm{C} \mathrm{NMR}\left(101 \mathrm{MHz}, \mathrm{CDCl}_{3}\right) \delta 168.5,158.2$, $153.5,145.9,140.4,135.6,132.5,129.5,128.5,123.8,120.5,24.2$. HRMS (ESI+, m/z): $[\mathrm{M}+\mathrm{H}]+$ calcd for $\mathrm{C}_{14} \mathrm{H}_{12} \mathrm{~N}_{3} \mathrm{O}^{+}: 238.0975$, found: 238.0995 .<smiles>O=c1nc(-c2ccccc2)nc2ccc(Cl)cn12</smiles>

\section{$3 w$}

7-Chloro-2-phenyl-4H-pyrido[1,2-a][1,3,5]triazin-4-one (3w): The reaction was set up inside an $\mathrm{N}_{2}$-filled glovebox according to the General Procedure using imine $1 w(43.3 \mathrm{mg}, 0.200 \mathrm{mmol}, 1.0$ equiv), and the reaction mixture was stirred at $60{ }^{\circ} \mathrm{C}$. Purification by flash-column chromatography over silica gel $(2 \%$ $\mathrm{MeOH} / \mathrm{CH}_{2} \mathrm{Cl}_{2}$ ) gave the desired product $3 \mathrm{w}$ as an off-white solid $\left(37 \mathrm{mg}, 72 \%\right.$ yield). $\mathrm{mp} 256-257^{\circ} \mathrm{C}$. IR (neat) 1704, 1621, 1509, 1467, 1374, 1304, 841, 777, 696, $537 \mathrm{~cm}^{-1} .{ }^{1} \mathrm{H}$ NMR $\left(500 \mathrm{MHz}, \mathrm{CDCl}_{3}\right) \delta 9.09$ (d, $J=2.4 \mathrm{~Hz}, 1 \mathrm{H}), 8.56(\mathrm{~d}, J=8.0 \mathrm{~Hz}, 2 \mathrm{H}), 7.94(\mathrm{dd}, J=9.3,2.4 \mathrm{~Hz}, 1 \mathrm{H}), 7.65(\mathrm{~d}, J=9.3 \mathrm{~Hz}, 1 \mathrm{H}), 7.57(\mathrm{t}, J$ $=7.6 \mathrm{~Hz}, 1 \mathrm{H}), 7.50(\mathrm{t}, J=8.3, \mathrm{~Hz}, 3 \mathrm{H}) .{ }^{13} \mathrm{C} \mathrm{NMR}\left(151 \mathrm{MHz}, \mathrm{CDCl}_{3}\right) \delta$ 169.8, 154.3, 150.4, 142.7, 135.6, 133.1, 129.8, 128.7, 128.0, 126.6, 126.0. HRMS (ESI+, m/z): $[\mathrm{M}+\mathrm{H}]+$ calcd for $\mathrm{C}_{13} \mathrm{H}_{9} \mathrm{CIN}_{3} \mathrm{O}^{+}: 258.0429$, found: 258.0450 . 
<smiles>O=c1nc(-c2ccccc2)nc2cc(C(F)(F)F)ccn12</smiles>

$3 x$

2-Phenyl-8-(trifluoromethyl)-4H-pyrido[1,2-a][1,3,5]triazin-4-one (3x): ${ }^{11}$ The reaction was set up inside a $\mathrm{N}_{2}$-filled glovebox according to the General Procedure using imine $1 \times$ ( $50.0 \mathrm{mg}, 0.200 \mathrm{mmol}, 1.0$ equiv), and the reaction mixture was stirred at $60{ }^{\circ} \mathrm{C}$. Purification by preparative thin-layer chromatography $(30 \%$

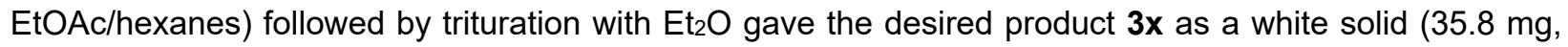
$62 \%$ yield). Spectroscopic data are in agreement with reported values. ${ }^{11}{ }^{1} \mathrm{H} \mathrm{NMR}\left(600 \mathrm{MHz}, \mathrm{CDCl}_{3}\right) \delta 9.15$ (d, $J=7.2 \mathrm{~Hz}, 1 \mathrm{H}), 8.59$ (d, $J=7.2 \mathrm{~Hz}, 2 \mathrm{H}), 7.94(\mathrm{~s}, 1 \mathrm{H}), 7.61(\mathrm{t}, J=7.3 \mathrm{~Hz}, 1 \mathrm{H}), 7.52(\mathrm{t}, J=7.7 \mathrm{~Hz}, 2 \mathrm{H})$, 7.40 (dd, $J=7.2,1.7 \mathrm{~Hz}, 1 \mathrm{H}) .{ }^{13} \mathrm{C}\left(151 \mathrm{MHz}, \mathrm{CDCl}_{3}\right) \delta 170.8,155.7,150.7,142.5(\mathrm{q}, J=35.3 \mathrm{~Hz}), 135.3$, 133.5, 131.8, 130.0, 128.8, 123.6 (q, $J=4.4 \mathrm{~Hz}$ ), 122.5 (q, $J=274.2 \mathrm{~Hz}), 112.7$ (q, $J=2.6 \mathrm{~Hz}$ ). ${ }^{19} \mathrm{~F} \mathrm{NMR}$ (376 MHz, $\left.\mathrm{CDCl}_{3}\right) \delta-65.9$.<smiles>Cc1ccc2nc(-c3ccccc3)nc(=O)n2n1</smiles>

\section{$3 y$}

7-Methyl-2-phenyl-4H-pyridazino[1,6-a][1,3,5]triazin-4-one (3y): The reaction was set up inside a $\mathrm{N}_{2}$ filled glovebox according to the General Procedure using imine 1y (39.4 mg, $0.200 \mathrm{mmol}, 1.0$ equiv), and the reaction mixture was stirred at $60{ }^{\circ} \mathrm{C}$. Purification by flash-column chromatography over silica gel $(4 \%$ $\mathrm{MeOH} / \mathrm{EtOAc}$ ) followed by trituration with acetone gave the desired product $\mathbf{3 y}$ as a white solid (32.8 $\mathrm{mg}$, $69 \%$ yield). $\mathrm{mp} 267^{\circ} \mathrm{C}$ (decomp). IR (neat) 3068, 1716, 1626, 1529, 1466, 1415, 1360, 848, 782, $709 \mathrm{~cm}^{-}$ 1. ${ }^{1} \mathrm{H}$ NMR $\left(600 \mathrm{MHz}, 50 \% \mathrm{CDCl}_{3}: \mathrm{MeOD}\right) \delta 8.49(\mathrm{dd}, J=8.4,1.2 \mathrm{~Hz}, 2 \mathrm{H}), 7.95(\mathrm{~d}, J=9.2 \mathrm{~Hz}, 1 \mathrm{H}), 7.84(\mathrm{~d}$, $J=9.2 \mathrm{~Hz}, 1 \mathrm{H}), 7.58(\mathrm{t}, J=7.4 \mathrm{~Hz}, 1 \mathrm{H}), 7.49(\mathrm{t}, J=7.7 \mathrm{~Hz}, 2 \mathrm{H}), 2.74(\mathrm{~s}, 3 \mathrm{H}) .{ }^{13} \mathrm{C} \mathrm{NMR}(151 \mathrm{MHz}, 50 \%$ $\left.\mathrm{CDCl}_{3}: \mathrm{MeOD}\right) \delta 170.4,158.0,154.9,153.0,135.6,135.5,134.0,133.8,130.2,129.2,22.1$. HRMS (ESI+, $\mathrm{m} / \mathrm{z}$ ): $[\mathrm{M}+\mathrm{H}]^{+}$calcd for $\mathrm{C}_{13} \mathrm{H}_{11} \mathrm{~N}_{4} \mathrm{O}^{+}: 239.0927$, found: 239.0939 .<smiles>Cn1ccn2c(=O)nc(-c3ccccc3)nc12</smiles>

8-Methyl-2-phenylimidazo[1,2-a][1,3,5]triazin-4(8H)-one (3z): The reaction was set up inside an $\mathrm{N}_{2}$-filled glovebox according to the General Procedure using imine $1 \mathrm{z}(37.0 \mathrm{mg}, 0.200 \mathrm{mmol}, 1.0$ equiv), and the reaction mixture was stirred at $60{ }^{\circ} \mathrm{C}$. Purification by flash-column chromatography over silica gel $(3 \%$ $\mathrm{MeOH} / \mathrm{EtOAc}$ ) gave the desired product $3 z$ as a white solid (32 mg, $71 \%$ yield). $\mathrm{mp} 231-232{ }^{\circ} \mathrm{C}$. IR (neat) $1675,1597,1509,1381,1284,1021,923,764,704,665 \mathrm{~cm}^{-1} .{ }^{1} \mathrm{H} \mathrm{NMR}\left(600 \mathrm{MHz}, \mathrm{CDCl}_{3}\right) \delta 8.50(\mathrm{dd}, J=$ 
8.4, $1.4 \mathrm{~Hz}, 2 \mathrm{H}), 7.52-7.48(\mathrm{~m}, 2 \mathrm{H}), 7.46-7.42(\mathrm{~m}, 2 \mathrm{H}), 6.98(\mathrm{~d}, J=2.6 \mathrm{~Hz}, 1 \mathrm{H}), 3.80(\mathrm{~s}, 3 \mathrm{H}) .{ }^{13} \mathrm{C}$ NMR $\left(151 \mathrm{MHz}, \mathrm{CDCl}_{3}\right) \delta 169.1,151.5,150.0,136.2,132.1,129.3,128.4,119.6,108.5,31.9$. HRMS (ESI+, m/z): $[\mathrm{M}+\mathrm{H}]+$ calcd for $\mathrm{C}_{12} \mathrm{H}_{11} \mathrm{~N}_{4} \mathrm{O}^{+}: 227.0927$, found: 227.0946 .

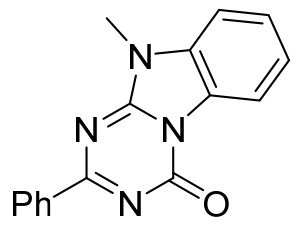

3aa

10-Methyl-2-phenylbenzo[4,5]imidazo[1,2-a][1,3,5]triazin-4(10H)-one (3aa): The reaction was set up inside a $\mathrm{N}_{2}$-filled glovebox according to the General Procedure using imine 1aa (47.1 mg, $0.200 \mathrm{mmol}, 1.0$ equiv), and the reaction was stirred at $60{ }^{\circ} \mathrm{C}$. Purification by preparative thin-layer chromatography $(5 \%$ acetone $/ \mathrm{CH}_{2} \mathrm{Cl}_{2}$ ) gave the desired product 3aa as a cream-colored solid $\left(49.0 \mathrm{mg}, 89 \%\right.$ yield). $\mathrm{mp} 230{ }^{\circ} \mathrm{C}$ (decomp). IR (neat) 3050, 1606, 1573, 1462, 1390, 1327, 764, 742, 706, $687 \mathrm{~cm}^{-1} .{ }^{1} \mathrm{H}$ NMR $(600 \mathrm{MHz}$, $\left.\mathrm{CDCl}_{3}\right) \delta 8.68-8.55(\mathrm{~m}, 3 \mathrm{H}), 7.58-7.54(\mathrm{~m}, 2 \mathrm{H}), 7.52-7.47(\mathrm{~m}, 3 \mathrm{H}), 7.45(\mathrm{~d}, J=8.1 \mathrm{~Hz}, 1 \mathrm{H}), 3.95(\mathrm{~s}$, $3 \mathrm{H}) .{ }^{13} \mathrm{C}$ NMR $\left(151 \mathrm{MHz}, \mathrm{CDCl}_{3}\right) \delta 170.5,152.90,152.86,136.1,132.6,131.0,129.7,128.5,126.9,125.2$, 124.6, 117.0, 109.3, 28.7. HRMS (ESI+, m/z): $[\mathrm{M}+\mathrm{H}]^{+}$calcd for $\mathrm{C}_{16} \mathrm{H}_{13} \mathrm{~N}_{4} \mathrm{O}^{+}:$277.1084, found: 277.1107 .

\section{VII: Procedures and characterization data for triazinone products 3 via three-component reaction}

\section{General three-component procedure for synthesis of triazinones}

In a $\mathrm{N}_{2}$-filled glovebox, an oven-dried $2-5 \mathrm{~mL}$ Biotage microwave vial (\#351521) was charged with [Cp* Rh(MeCN) $\left.)_{3}\right]\left(\mathrm{SbF}_{6}\right)_{2}(10 \mathrm{~mol} \%, 16.7 \mathrm{mg}, 0.020 \mathrm{mmol}), \mathrm{PivOH}(20.4 \mathrm{mg}, 0.200 \mathrm{mmol}, 1.0$ equiv), HFIP $(1.0 \mathrm{~mL}, 0.2 \mathrm{M})$, aldehyde $(0.400 \mathrm{mmol}, 2$ equiv), amine $(0.200 \mathrm{mmol}, 1.0$ equiv), carbamylation reagent 2 ( $56.8 \mathrm{mg}, 0.300 \mathrm{mmol}, 1.5$ equiv), and $3 \AA$ molecular sieves $(200 \mathrm{mg})$. The vial was sealed with a teflonlined septum and removed from the glovebox. The solution was heated with stirring for $20 \mathrm{~h}$ at $80{ }^{\circ} \mathrm{C}$ in a pre-equilibrated oil bath. After cooling to rt, the crude mixture was filtered through a pad of celite with EtOAc and was concentrated in vacuo. The products were purified by the indicated chromatography techniques.<smiles>O=c1nc(-c2ccccc2)nc2ccccn12</smiles>

$3 a$

2-Phenyl-4H-pyrido[1,2-a][1,3,5]triazin-4-one (3a): The reaction was set up according to the General Procedure using benzaldehyde $(42.4 \mathrm{mg}, 0.400 \mathrm{mmol}, 2.0$ equiv) and 2-aminopyridine (18.8 $\mathrm{mg}, 0.200$ $\mathrm{mmol}, 1.0$ equiv) . Purification by flash-column chromatography over silica gel (50\% EtOAc/hexanes) gave the desired product $3 \mathrm{a}$ as a white solid $\left(31 \mathrm{mg}, 70 \%\right.$ yield). ${ }^{1} \mathrm{H}$ and ${ }^{13} \mathrm{C}$ NMR spectra matched with $3 a$ obtained from the two-component conditions above. 
<smiles>COc1ccc(-c2nc(=O)n3ccccc3n2)cc1</smiles>

3b

2-(4-Methoxyphenyl)-4H-pyrido[1,2-a][1,3,5]triazin-4-one (3b): The reaction was set up according to the General Procedure using 4-methoxybenzaldehyde (54.5 mg, $0.400 \mathrm{mmol}, 2.0$ equiv) and 2-aminopyridine (18.8 mg, $0.200 \mathrm{mmol}, 1.0$ equiv). Purification by flash-column chromatography over silica gel (70\% EtOAc/hexanes) gave the desired product $3 \mathrm{~b}$ as an off-white solid ( $38 \mathrm{mg}, 75 \%$ yield). ${ }^{1} \mathrm{H}$ and ${ }^{13} \mathrm{C} \mathrm{NMR}$ spectra matched with $\mathbf{3 b}$ obtained from the two-component conditions above.<smiles>O=c1nc(-c2ccc(C(F)(F)F)cc2)nc2ccccn12</smiles>

3d

2-(4-(Trifluoromethyl)phenyl)-4H-pyrido[1,2-a][1,3,5]triazin-4-one $(3 \mathrm{~d})$ : The reaction was set up according to the General Procedure using 4-(trifluoromethyl)benzaldehyde (69.6 mg, $0.400 \mathrm{mmol}, 2.0$ equiv) and 2-aminopyridine ( $18.8 \mathrm{mg}, 0.200 \mathrm{mmol}, 1.0$ equiv). Purification by flash-column chromatography over silica gel ( $70 \%$ EtOAc/hexanes) gave the desired product $3 \mathrm{~d}$ as a yellow solid $\left(44 \mathrm{mg}, 76 \%\right.$ yield). ${ }^{1} \mathrm{H}$ and ${ }^{13} \mathrm{C}$ NMR spectra matched with $3 \mathrm{~d}$ obtained from the two-component conditions above.<smiles>Cn1cc(-c2nc(=O)n3ccccc3n2)cn1</smiles>

3k

2-(1-Methyl-1H-pyrazol-4-yl)-4H-pyrido[1,2-a][1,3,5]triazin-4-one (3k): The reaction was set up according to the General Procedure using 1-methylpyrazole-4-carbaldehyde $(44.0 \mathrm{mg}, 0.400 \mathrm{mmol}, 2.0$ equiv) and 2-aminopyridine (18.8 mg, $0.200 \mathrm{mmol}, 1.0$ equiv). Purification by flash-column chromatography over silica gel $(10 \% \mathrm{MeOH} / \mathrm{EtOAc})$ gave the desired product $3 \mathbf{k}$ as a white solid $\left(40 \mathrm{mg}, 88 \%\right.$ yield). ${ }^{1} \mathrm{H}$ and ${ }^{13} \mathrm{C}$ NMR spectra matched with $\mathbf{3 k}$ obtained from the two-component conditions above. 
<smiles>Cc1ccc2nc(-c3ccccc3)nc(=O)n2n1</smiles>

3y

7-Methyl-2-phenyl-4H-pyridazino[1,6-a][1,3,5]triazin-4-one (3y): The reaction was set up according to the General Procedure using benzaldehyde $(42.4 \mathrm{mg}, 0.400 \mathrm{mmol}, 2.0$ equiv) and 6-methylpyridazin-3amine (21.8 mg, $0.200 \mathrm{mmol}, 1.0$ equiv). Purification by silica gel chromatography (4\% MeOH/EtOAc) afforded 3y as a cream-colored solid (19.6 mg, $41 \%$ yield). ${ }^{1} \mathrm{H}$ and ${ }^{13} \mathrm{C}$ NMR spectra matched with $3 y$ obtained from the two-component conditions above.<smiles>Cn1c2ccccc2n2c(=O)nc(-c3ccccc3)nc12</smiles>

\section{3aa}

10-Methyl-2-phenylbenzo[4,5]imidazo[1,2-a][1,3,5]triazin-4(10H)-one (3aa): The reaction was set up according to the General Procedure using benzaldehyde (42.4 mg, $0.400 \mathrm{mmol}, 2.0$ equiv) and 1methylbenzimidazol-2-amine $(29.4 \mathrm{mg}, 0.200 \mathrm{mmol}, 1.0$ equiv). Purification by preparative thin layer chromatography (60\% EtOAc/hexanes) gave the desired product 3aa as a light yellow solid (24 mg, 43\% yield). ${ }^{1} \mathrm{H}$ and ${ }^{13} \mathrm{C}$ NMR spectra matched with 3aa obtained from the two-component conditions above.

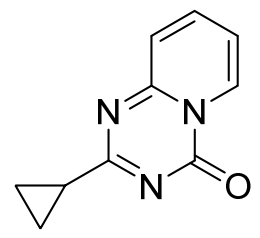

$3 q$

2-Cyclopropyl-4H-pyrido[1,2-a][1,3,5]triazin-4-one (3q): The reaction was set up according to the General Procedure using cyclopropanecarbaldehyde (28.0 mg, $0.400 \mathrm{mmol}, 2.0$ equiv) and 2aminopyridine (18.8 mg, $0.200 \mathrm{mmol}, 1.0$ equiv). Purification by flash-column chromatography over silica gel $\left(90 \%\right.$ EtOAc/hexanes) gave the desired product $3 q$ as a white solid $\left(27 \mathrm{mg}, 72 \%\right.$ yield). ${ }^{1} \mathrm{H}$ and ${ }^{13} \mathrm{C}$ NMR spectra matched with $\mathbf{3 q}$ obtained from the two-component conditions above. 


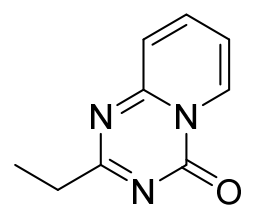

$3 a b$

2-Ethyl-4H-pyrido[1,2-a][1,3,5]triazin-4-one (3ab): The reaction was set up according to the General Procedure using propionaldehyde $(23.2 \mathrm{mg}, 0.400 \mathrm{mmol}, 2.0$ equiv) and 2-aminopyridine (18.8 mg, 0.200 mmol, 1.0 equiv). Purification by flash-column chromatography over silica gel ( $5 \% \mathrm{MeOH} / \mathrm{EtOAc}$ ) gave the desired product $3 \mathbf{a b}$ as a white solid $\left(13 \mathrm{mg}, 37 \%\right.$ yield). $\mathrm{mp} 92-93^{\circ} \mathrm{C}$. IR (neat) $1703,1636,1519,1413$, 1289, 1183, 1100, 949, 862, $781 \mathrm{~cm}^{-1} .{ }^{1} \mathrm{H}$ NMR $\left(600 \mathrm{MHz}, \mathrm{CDCl}_{3}\right) \delta 9.03$ (dd, J = 7.1, $\left.1.6 \mathrm{~Hz}, 1 \mathrm{H}\right), 8.03$ (td, $J=7.8,1.1 \mathrm{~Hz}, 1 \mathrm{H}), 7.60(\mathrm{~d}, J=8.8 \mathrm{~Hz}, 1 \mathrm{H}), 7.33(\mathrm{t}, J=6.9,1 \mathrm{H}), 2.80(\mathrm{q}, J=7.6 \mathrm{~Hz}, 2 \mathrm{H}), 1.35(\mathrm{t}, J=7.6$ $\mathrm{Hz}, 3 \mathrm{H}) .{ }^{13} \mathrm{C}$ NMR $\left(151 \mathrm{MHz}, \mathrm{CDCl}_{3}\right) \delta 179.9,155.3,151.2,141.9,129.8,125.0,118.0,33.3,11.8$. HRMS $(\mathrm{ESI}+, \mathrm{m} / \mathrm{z}):[\mathrm{M}+\mathrm{H}]+$ calcd for $\mathrm{C}_{9} \mathrm{H}_{10} \mathrm{~N}_{3} \mathrm{O}^{+}:$176.0818, found: 176.0835 .<smiles>O=c1nc(C2CCOCC2)nc2ccccn12</smiles>

3ac

2-(Tetrahydro-2H-pyran-4-yl)-4H-pyrido[1,2-a][1,3,5]triazin-4-one (3ac): The reaction was set up according to the General Procedure using tetrahydro- $2 \mathrm{H}$-pyran-4-carbaldehyde $(45.7 \mathrm{mg}, 0.400 \mathrm{mmol}, 2.0$ equiv) and 2-aminopyridine ( $18.8 \mathrm{mg}, 0.200 \mathrm{mmol}, 1.0$ equiv). Purification by flash-column chromatography over silica gel ( $5 \% \mathrm{MeOH} / \mathrm{EtOAc}$ ) gave the desired product 3ac as a white solid (45 mg, $97 \%$ yield). $\mathrm{mp}$ 149-152 ${ }^{\circ} \mathrm{C}$. IR (neat) 3109, 2949, 2860, 1707, 1625, 1519, 1412, 1302, 1235, 1111, $787 \mathrm{~cm}^{-1} .{ }^{1} \mathrm{H}$ NMR $\left(500 \mathrm{MHz}, \mathrm{CDCl}_{3}\right) \delta 9.01$ (dd, $\left.J=7.0,1.6 \mathrm{~Hz}, 1 \mathrm{H}\right), 8.03$ (td, $\left.J=8.7,6.9 \mathrm{~Hz}, 1 \mathrm{H}\right), 7.59$ (d, $J=8.8 \mathrm{~Hz}, 1 \mathrm{H}$ ), 7.34 (td, $J=6.9,1.4 \mathrm{~Hz}, 1 \mathrm{H}$ ), 4.02 (dd, $J=11.5,4.3 \mathrm{~Hz}, 2 \mathrm{H}$ ), $3.48(\mathrm{td}, J=11.6,2.4 \mathrm{~Hz}, 2 \mathrm{H}), 2.92-2.86$ $(\mathrm{m}, 1 \mathrm{H}), 2.06-1.83(\mathrm{~m}, 4 \mathrm{H}) .{ }^{13} \mathrm{C}$ NMR $\left(151 \mathrm{MHz}, \mathrm{CDCl}_{3}\right) \delta 179.8,155.4,151.3,141.9,129.8,125.1,118.1$, 67.6, 44.6, 30.5. HRMS (ESI+, m/z): [M+H] + calcd for $\mathrm{C}_{12} \mathrm{H}_{13} \mathrm{~N}_{3} \mathrm{O}_{2}{ }^{+}:$232.1081, found: 232.1099.<smiles>CC(C)(C)OC(=O)N1CCC(c2nc(=O)n3ccccc3n2)CC1</smiles>

3ad

tert-Butyl 4-(4-oxo-4H-pyrido[1,2-a][1,3,5]triazin-2-yl)piperidine-1-carboxylate (3ad): The reaction was set up according to the General Procedure using tert-butyl 4-formylpiperidine-1-carboxylate (85.3 mg, 
$0.400 \mathrm{mmol}, 2.0$ equiv) and 2-aminopyridine (18.8 mg, $0.200 \mathrm{mmol}, 1.0$ equiv). Purification by flash-column chromatography over silica gel (5\% MeOH/EtOAc) gave the desired product 3ad as a white solid (55 mg, $83 \%$ yield). $\mathrm{mp} 171-173{ }^{\circ} \mathrm{C}$. IR (neat) $1716,1676,1628,1564,1519,1419,1364,1228,1162,973,789$ $\mathrm{cm}^{-1} .{ }^{1} \mathrm{H}$ NMR $\left(600 \mathrm{MHz}, \mathrm{CDCl}_{3}\right) \delta 9.00(\mathrm{~d}, J=6.9 \mathrm{~Hz}, 1 \mathrm{H}), 8.02(\mathrm{td}, J=8.7,6.8 \mathrm{~Hz}, 1 \mathrm{H}), 7.57(\mathrm{~d}, J=8.7$ $\mathrm{Hz}, 1 \mathrm{H}), 7.33(\mathrm{td}, J=6.9,1.2 \mathrm{~Hz}, 1 \mathrm{H}), 4.16$ (broad s, 2H), $2.81-2.76(\mathrm{~m}, 3 \mathrm{H}), 1.98-1.88(\mathrm{~m}, 2 \mathrm{H}), 1.81$ (qd, $J=12.4,4.3 \mathrm{~Hz}, 2 \mathrm{H}), 1.42(\mathrm{~s}, 9 \mathrm{H}) .{ }^{13} \mathrm{C}$ NMR $(151 \mathrm{MHz}, \mathrm{CDCl} 3) \delta 180.0,155.4,154.8,151.2,141.9$, 129.8, 125.1, 118.1, 79.5, 45.7, 44.0, 43.2, 29.8, 28.5. HRMS (ESI+, m/z): $[\mathrm{M}+\mathrm{H}]+$ calcd for $\mathrm{C}_{17} \mathrm{H}_{23} \mathrm{~N}_{4} \mathrm{O}_{3}{ }^{+}$: 331.1765, found: 331.1748 .

\section{VIII: Procedure and characterization data for $1.0 \mathrm{mmol}$ scale three-component reaction}

Three-component procedure for synthesis of 2-(tetrahydro-2H-pyran-4-yl)-4H-pyrido[1,2a][1,3,5]triazin-4-one (3ac) $(1.0 \mathrm{mmol}$ scale):

In a $\mathrm{N}_{2}$-filled glovebox, a 10-20 mL Biotage microwave vial (\#354833) was charged with [Cp* Rh(MeCN) $\left.)_{3}\right]\left(\mathrm{SbF}_{6}\right)_{2}(5 \mathrm{~mol} \%, 41.6 \mathrm{mg}, 0.050 \mathrm{mmol}), \mathrm{PivOH}$ (102 mg, $1.00 \mathrm{mmol}, 1.0$ equiv), HFIP (5.0 $\mathrm{mL}, 0.2 \mathrm{M}$ ), tetrahydro-2H-pyran-4-carbaldehyde (228 mg, $2.00 \mathrm{mmol}, 2$ equiv), 2-aminopyridine (94.1 mg $1.00 \mathrm{mmol}, 1.0$ equiv), carbamylation reagent 2 (284 mg, $1.50 \mathrm{mmol}, 1.5$ equiv), and $3 \AA$ molecular sieves $(1.0 \mathrm{~g})$. The vial was sealed with a teflon-lined septum and removed from the glovebox. The solution was heated with stirring for $20 \mathrm{~h}$ at $80^{\circ} \mathrm{C}$ in a pre-equilibrated oil bath. After cooling to rt, the crude mixture was filtered through a pad of celite, washed with EtOAc, and concentrated in vacuo. Purification by flash-column chromatography over silica gel $(5 \% \mathrm{MeOH} / \mathrm{EtOAc})$ gave the desired product 3ac as a white solid (207 mg, $90 \%$ yield). ${ }^{1} \mathrm{H}$ and ${ }^{13} \mathrm{C}$ NMR spectra matched with 3ac obtained from the three-component conditions above.

\section{IX: Procedure and characterization data for experiments with rhodacycle 6}

\section{Formation of rhodacycle 6}<smiles>C(=N/c1ccccn1)\c1ccccc1</smiles>

$1 \mathrm{a}$

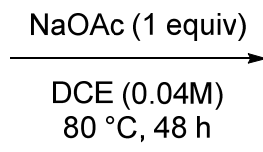

$$
\begin{aligned}
& {\left[\mathrm{Cp}^{*} \mathrm{RhCl}_{2}\right]_{2}} \\
& \text { (0.5 equiv) }
\end{aligned}
$$

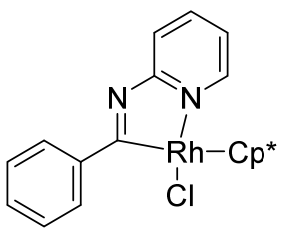

6

In a $\mathrm{N}_{2}$-filled glovebox, an oven-dried 10-20 mL Biotage microwave vial (\#354833) was charged with imine 1a $(91.1 \mathrm{mg}, 0.500 \mathrm{mmol}, 1.0 \text { equiv), [Cp* RhCl}]_{2}(155 \mathrm{mg}, 0.250 \mathrm{mmol}, 0.50$ equiv), NaOAc (41.0 mg, $0.500 \mathrm{mmol}, 1.0$ equiv), and DCE $(12.5 \mathrm{~mL})$. The vial was sealed with a teflon-lined septum and removed from the glovebox, and was heated for $48 \mathrm{~h}$ at $80^{\circ} \mathrm{C}$ in a pre-equilibrated oil bath. The solution was then cooled to rt, filtered through a pad of celite inside the glovebox, and concentrated slowly under a stream of $\mathrm{N}_{2}$. The crude material was purified by preparative thin-layer chromatography $(80 \% \mathrm{EtOAc} / \mathrm{hexanes})$ followed by recrystallization $\left(\mathrm{CH}_{2} \mathrm{Cl}_{2} / \mathrm{EtOAc} /\right.$ pentane) to yield the desired product 6 as a red solid (10.0 mg, $4 \%$ yield). IR (neat) 2920, 2853, 1675, 1606, 1492, 1448, 1303, 1260, 1208, 1025, 918, 780, $698 \mathrm{~cm}^{-1} .{ }^{1} \mathrm{H}$ NMR $\left(600 \mathrm{MHz}, \mathrm{CDCl}_{3}\right) \delta 8.45(\mathrm{~d}, J=5.6 \mathrm{~Hz}, 1 \mathrm{H}), 7.89-7.82(\mathrm{~m}, 2 \mathrm{H}), 7.78-7.71(\mathrm{~m}, 2 \mathrm{H}), 7.42-7.34$ $(\mathrm{m}, 3 \mathrm{H}), 7.05$ (td, $J=5.9,2.6 \mathrm{~Hz}, 1 \mathrm{H}), 1.43(\mathrm{~s}, 15 \mathrm{H}) .{ }^{13} \mathrm{C}$ NMR $\left(151 \mathrm{MHz}, \mathrm{CDCl}_{3}\right) \delta 170.1,149.3,146.5$, 
139.0, 129.5, 127.70, 127.68, 120.3, 119.7, 98.2 (d, $J=5.7 \mathrm{~Hz}), 9.1$. HRMS (ESI+, m/z): $[\mathrm{M}+\mathrm{H}]^{+}$calcd for $\mathrm{C}_{22} \mathrm{H}_{25} \mathrm{CIN}_{2} \mathrm{Rh}^{+}: 455.0756$, found: 455.0747 . [See X-ray crystallographic data below]

\section{Reaction of imine 1 a with 2 using 6 as the catalyst}

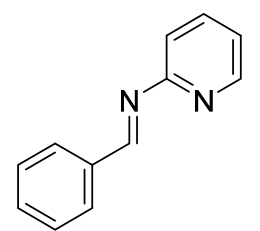

1a

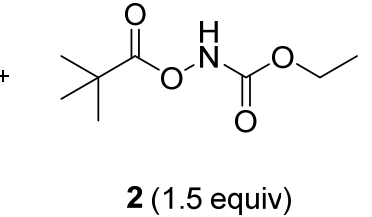

2 (1.5 equiv)

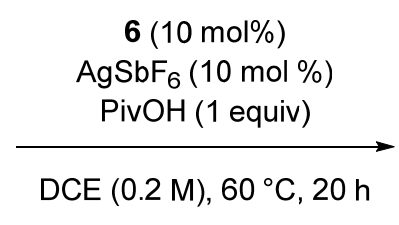

In a $\mathrm{N}_{2}$-filled glovebox, an oven-dried $2-5 \mathrm{~mL}$ Biotage microwave vial (\#351521) was charged with rhodacycle 6 ( $4.6 \mathrm{mg}, 0.010 \mathrm{mmol}, 0.10$ equiv), AgSbF 6 ( $3.4 \mathrm{mg}, 0.010 \mathrm{mmol}, 0.1$ equiv), PivOH (10.2 mg, $0.100 \mathrm{mmol}, 1.0$ equiv), DCE ( $0.5 \mathrm{~mL}, 0.2 \mathrm{M})$, imine $1 \mathrm{a}(18.2 \mathrm{mg}, 0.100 \mathrm{mmol}, 1.0$ equiv) and carbamylation reagent 2 (28.4 mg, $0.150 \mathrm{mmol}, 1.5$ equiv). The vial was sealed with a teflon-lined septum, removed from the glovebox, and was heated for $20 \mathrm{~h}$ at $60^{\circ} \mathrm{C}$ in a pre-equilibrated oil bath. After cooling to rt, the reaction mixture was transferred to a vial with EtOAc and was concentrated in vacuo. Purification by preparative thin-layer chromatography $(50 \%$ EtOAc/hexanes) followed by a second preparative thin-layer chromatography $\left(4 \% \mathrm{MeOH} / \mathrm{CH}_{2} \mathrm{Cl}_{2}\right)$ purification to remove residual impurities gave the desired product $3 \mathbf{a}$ as a white solid ( $14.2 \mathrm{mg}, 64 \%$ yield). Spectroscopic data agreed with that reported above.

\section{X: Procedure and characterization data for experiments with uncyclized intermediate 7}

\section{Formation of uncyclized intermediate 7}<smiles>Brc1ccccc1/N=C/c1ccccn1</smiles><smiles></smiles>

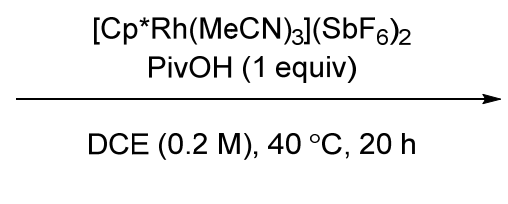

In a $\mathrm{N}_{2}$-filled glovebox, an oven-dried 10-20 mL Biotage microwave vial (\#354833) was charged with $\left[\mathrm{Cp}{ }^{*} \mathrm{Rh}(\mathrm{MeCN})_{3}\right]\left(\mathrm{SbF}_{6}\right)_{2}(10 \mathrm{~mol} \%, 146 \mathrm{mg}, 0.175 \mathrm{mmol})$, PivOH (179 mg, $1.75 \mathrm{mmol}, 1.0$ equiv), DCE ( $8.8 \mathrm{~mL}, 0.2 \mathrm{M}$ ), imine $1 \mathrm{f}$ (457 mg, $1.75 \mathrm{mmol}, 1.0$ equiv) and carbamylation reagent 2 (497 mg, $2.63 \mathrm{mmol}$, 1.5 equiv). The vial was sealed with a teflon-lined septum and removed from the glovebox. The reaction mixture was heated for $20 \mathrm{~h}$ at $40{ }^{\circ} \mathrm{C}$ in a pre-equilibrated oil bath. After cooling to rt, the reaction mixture was transferred to a vial with EtOAc and was concentrated in vacuo. Purification by flash-column chromatography over silica gel (20\% EtOAc/pentane) followed by preparative thin-layer chromatography (30\% $\mathrm{Et}_{2} \mathrm{O} /$ hexanes) gave the desired product 7 as a white solid (155 mg, $25 \%$ yield). $\mathrm{mp} 101-103{ }^{\circ} \mathrm{C}$. IR (neat) 2982, 1712, 1638, 1577, 1538, 1436, 1340, 1293, 1216, 1135, 1055, 769, 731, $607 \mathrm{~cm}^{-1} .{ }^{1} \mathrm{H}$ NMR $\left(400 \mathrm{MHz}, \mathrm{CDCl}_{3}\right) \delta 13.34$ (br s, 1H), $8.45(\mathrm{~s}, 1 \mathrm{H}), 7.76(\mathrm{t}, J=7.3 \mathrm{~Hz}, 1 \mathrm{H}), 7.59(\mathrm{~d}, J=8.0 \mathrm{~Hz}, 1 \mathrm{H}), 7.54-$ $7.33(\mathrm{~m}, 3 \mathrm{H}), 7.31-7.26(\mathrm{~m}, 1 \mathrm{H}), 7.13(\mathrm{t}, J=5.5 \mathrm{~Hz}, 1 \mathrm{H}), 4.12(\mathrm{q}, J=7.1 \mathrm{~Hz}, 2 \mathrm{H}), 1.22(\mathrm{t}, J=7.1 \mathrm{~Hz}, 3 \mathrm{H})$. ${ }^{13} \mathrm{C} \mathrm{NMR}\left(101 \mathrm{MHz}, \mathrm{CDCl}_{3}\right) \delta 160.4,154.6,152.0,146.3,138.70,138.68,132.5,130.3,129.4,127.4,124.1$, 121.1, 120.7, 62.1, 14.4. HRMS (ESI+, m/z): $[\mathrm{M}+\mathrm{H}]+$ calcd for $\mathrm{C}_{15} \mathrm{H}_{15} \mathrm{BrN}_{3} \mathrm{O}_{2}{ }^{+}: 348.0342$, found: 348.0339 . 


\section{Formation of $3 \mathrm{f}$ from uncyclized intermediate 7:}

In a $\mathrm{N}_{2}$-filled glovebox, an oven-dried $2-5 \mathrm{~mL}$ Biotage microwave vial (\#351521) was charged with $\left[\mathrm{Cp}^{*} \mathrm{Rh}(\mathrm{MeCN})_{3}\right]\left(\mathrm{SbF}_{6}\right)_{2}(10 \mathrm{~mol} \%, 8.3 \mathrm{mg}, 0.0100 \mathrm{mmol}), \mathrm{PivOH}(10.2 \mathrm{mg}, 0.100 \mathrm{mmol}, 1.0$ equiv), DCE $(0.5 \mathrm{~mL}, 0.2 \mathrm{M})$, and uncyclized intermediate $7(34.8 \mathrm{mg}, 0.100 \mathrm{mmol}, 1.0$ equiv). The vial was sealed with a teflon-lined septum, removed from the glovebox, and was heated for $20 \mathrm{~h}$ at $80{ }^{\circ} \mathrm{C}$ in a pre-equilibrated oil bath. After cooling to $\mathrm{rt}$, the reaction mixture was transferred to a vial with EtOAc and was concentrated in vacuo. The concentrate was dissolved in $\mathrm{CDCl}_{3}$ and $0.200 \mathrm{~mL}$ of a $0.5 \mathrm{M}$ solution of trimethyl(phenyl)silane in $\mathrm{CDCl}_{3}$ was added as an external standard.

Table S5. Reaction of uncyclized intermediate 7 to form $3 f$<smiles>CCOC(=O)N/C(=N\c1ccccn1)c1ccccc1Br</smiles>

7

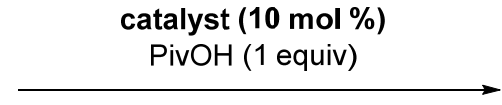

$\operatorname{DCE}(0.2 \mathrm{M}), 80^{\circ} \mathrm{C}, 20 \mathrm{~h}$

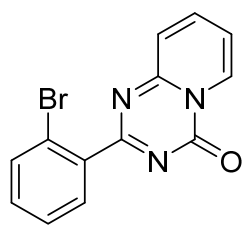

$3 \mathbf{f}$

\begin{tabular}{llc}
\hline Entry & catalyst & Yield $\mathbf{3 f}^{\mathrm{b}}(\%)$ \\
\hline 1 & none & $79 \%$ \\
2 & {$\left[\mathrm{Cp}{ }^{*} \mathrm{Rh}(\mathrm{MeCN})_{3}\right]\left(\mathrm{SbF}_{6}\right)_{2}$} & $79 \%$ \\
\hline
\end{tabular}

a. Conditions: $7(0.1 \mathrm{mmol})$ b. Yield determined by ${ }^{1} \mathrm{H}$ NMR relative to trimethyl(phenyl)silane as an external standard.

\section{Crude ${ }^{1} \mathrm{H}$ NMR spectra for reaction of uncyclized intermediate 7 to form $3 \mathrm{f}$}<smiles>O=c1nc(-c2ccccc2Br)nc2ccccn12</smiles>

3f

Crude ${ }^{1} \mathrm{H}$ NMR (entry 1$)$

$\mathrm{CDCl}_{3}$ $400 \mathrm{MHz}$

\section{TMSPh standard}
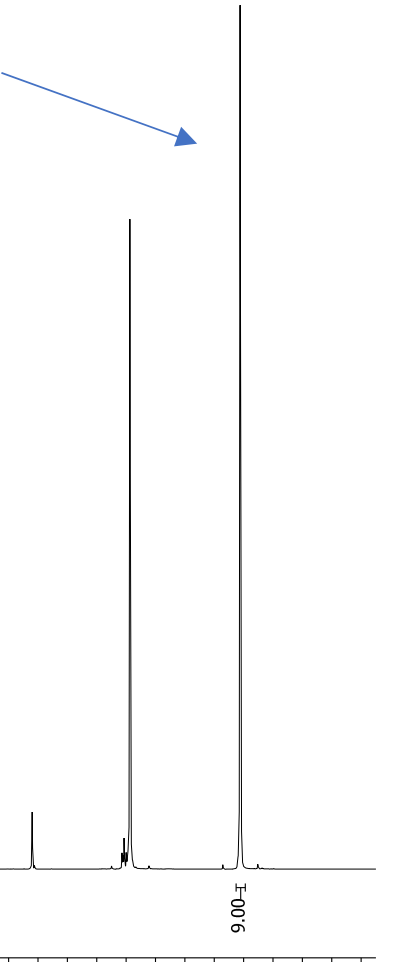

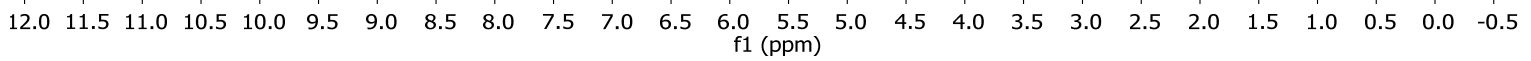




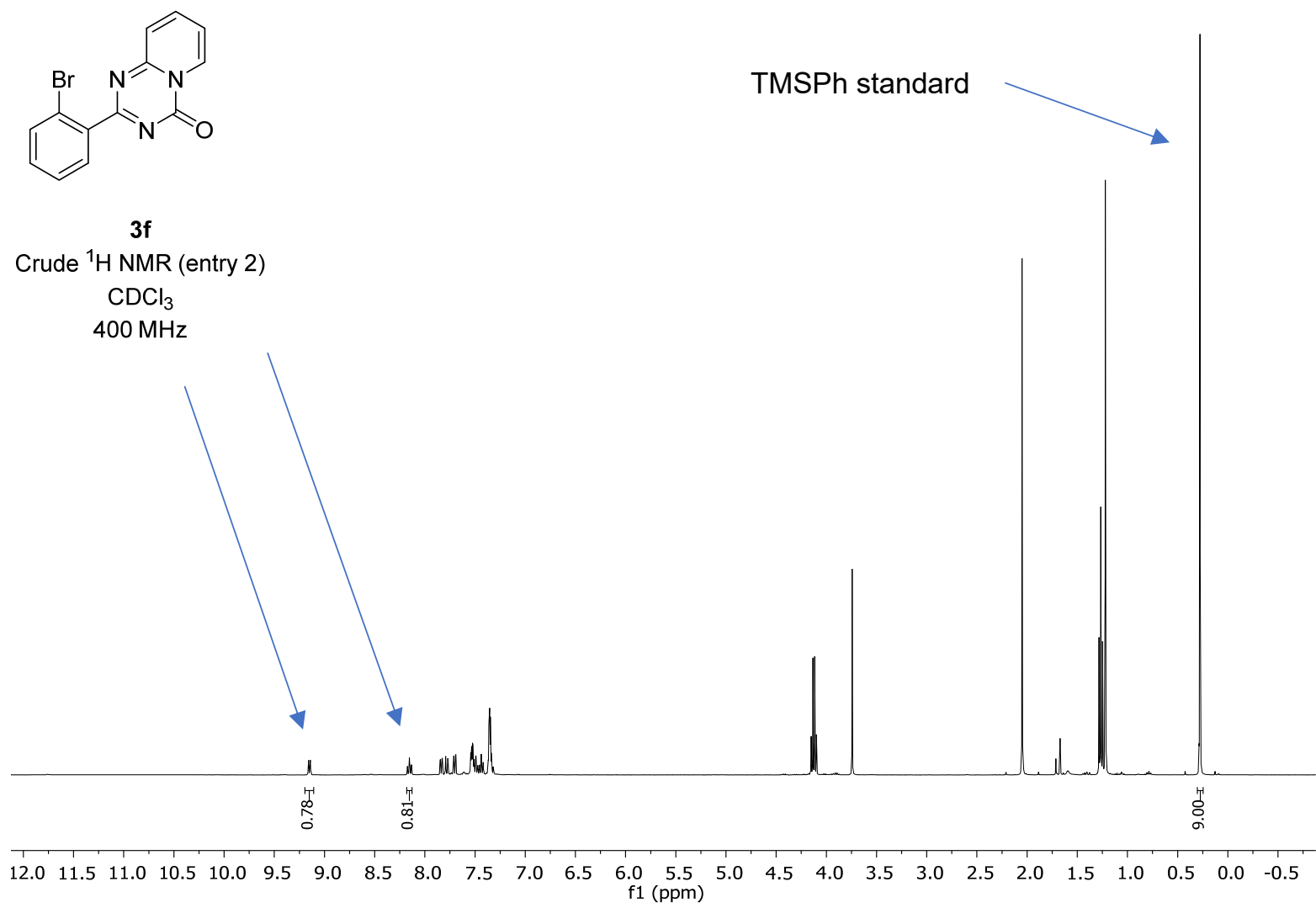

\section{References:}

1. Grohmann, C.; Wang, H.; Glorius, F., Rh[III]-Catalyzed C-H Amidation Using Aroyloxycarbamates To Give N-Boc Protected Arylamines. Org. Lett. 2013, 15, 3014-3017.

2. McLaughlin, E. C.; Shrestha, A.; Fletcher, M. H.; Steinauer, N. S.; Shinn, M. K.; Shahid, S. M., A Solvent-Free Amidation of Vinylogous Esters via Direct Aziridination. Tetrahedron Lett. 2013, 54, 54615463.

3. Xie, W.; Yoon, J. H.; Chang, S., (NHC)Cu-Catalyzed Mild C-H Amidation of (Hetero)arenes with Deprotectable Carbamates: Scope and Mechanistic Studies. J. Am. Chem. Soc. 2016, 138, 12605-12614.

4. Grohmann, C.; Wang, H.; Glorius, F., Rh[III]-Catalyzed Direct C-H Amination Using NChloroamines at Room Temperature. Org. Lett. 2012, 14, 656-659.

5. Schaufelberger, F.; Timmer, B. J. J.; Ramström, O., Resolving a Reactive Organometallic Intermediate from Dynamic Directing Group Systems by Selective C-H Activation. Chem. Eur. J. 2018, 24, 101-104.

6. Park, Y.; Park, K. T.; Kim, J. G.; Chang, S., Mechanistic Studies on the Rh(III)-Mediated Amido Transfer Process Leading to Robust $\mathrm{C}-\mathrm{H}$ Amination with a New Type of Amidating Reagent. J. Am. Chem. Soc. 2015, 137, 4534-4542. 
7. Younis, O.; El-Katori, E. E.; Hassanien, R.; Abousalem, A. S.; Tsutsumi, O., Luminescent Coatings: White-Color Luminescence from a Simple and Single Chromophore with High Anticorrosion Efficiency. Dyes and Pigments 2020, 175, 108146.

8. Montalvo-González, R.; Ariza-Castolo, A., Molecular Structure of Di-Aryl-Aldimines by Multinuclear Magnetic Resonance and X-Ray Diffraction. J. Mol. Struct. 2003, 655, 375-389.

9. Wu, C.; Qin, X.; Moeljadi, A. M. P.; Hirao, H.; Zhou, J. S., Copper-Catalyzed Asymmetric Arylation of $\mathrm{N}$-Heteroaryl Aldimines: Elementary Step of a 1,4-Insertion. Angew. Chem. Int. Ed. 2019, 58, 2705-2709.

10. Takeda Chemical Industries, L. U., 1996, A.

11. Xia, M.; Hu, W.; Sun, S.; Yu, J.-T.; Cheng, J., The Dearomative Annulation between N-2Pyridylamidine and $\mathrm{CO}_{2}$ toward Pyrido[1,2-a]-1,3,5-triazin-4-ones. Org. Biomol. Chem. 2017, 15, 40644067. 
XII. ${ }^{1} \mathrm{H},{ }^{13} \mathrm{C}$, and ${ }^{19} \mathrm{~F}$ NMR Spectra

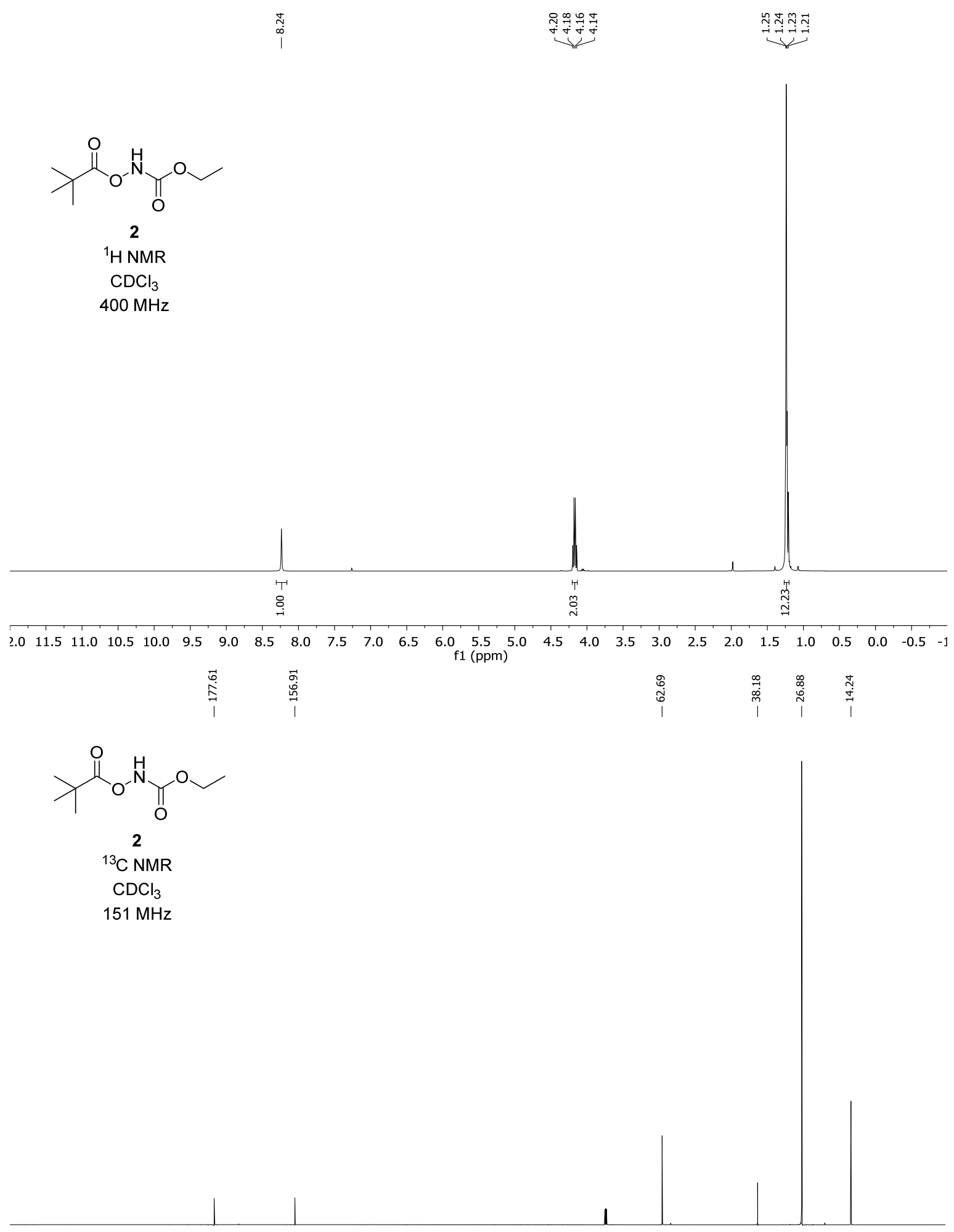

$\begin{array}{lllllllllllllllllllllllllll} & 220 & 210 & 200 & 190 & 180 & 170 & 160 & 150 & 140 & 130 & 120 & 110 & 100 & 90 & 80 & 70 & 60 & 50 & 40 & 30 & 20 & 10 & 0 & -\end{array}$ 


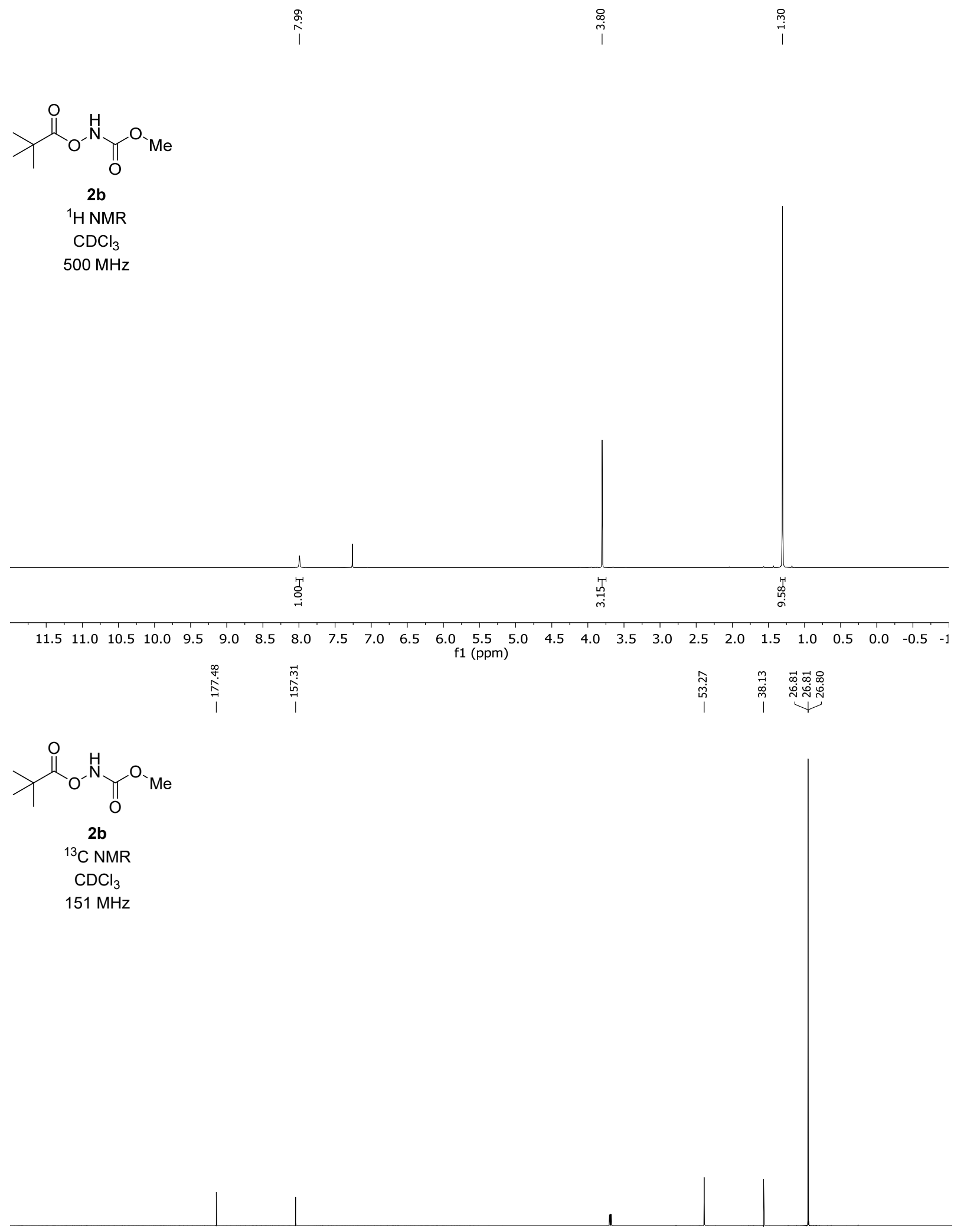

${ }^{13}{ }_{151}^{2 b}$ 


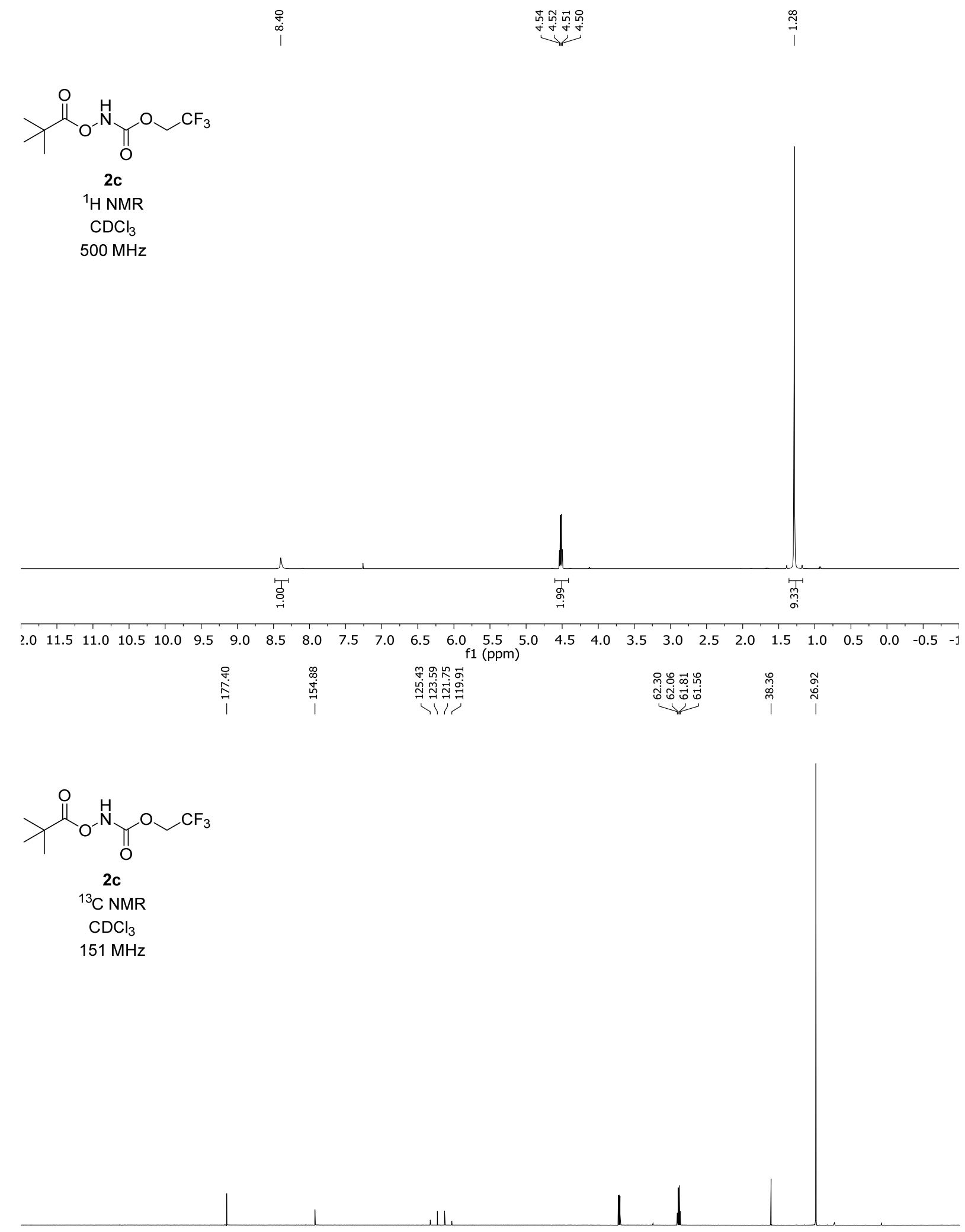

$\begin{array}{lllllllllllllllllllllllllllllll}30 & 220 & 210 & 200 & 190 & 180 & 170 & 160 & 150 & 140 & 130 & 120 & 110 & 100 & 90 & 80 & 70 & 60 & 50 & 40 & 30 & 20 & 10 & 0 & -:\end{array}$ 


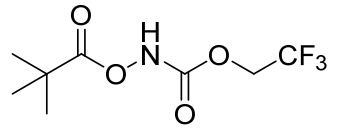

2c

${ }^{19} \mathrm{~F}$ NMR

$\mathrm{CDCl}_{3}$

$376 \mathrm{MHz}$

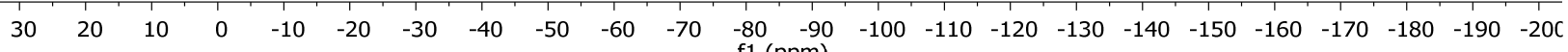
f1 (ppm) 


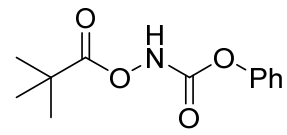

2d

${ }^{1} \mathrm{H}$ NMR

$\mathrm{CDCl}_{3}$

$600 \mathrm{MHz}$

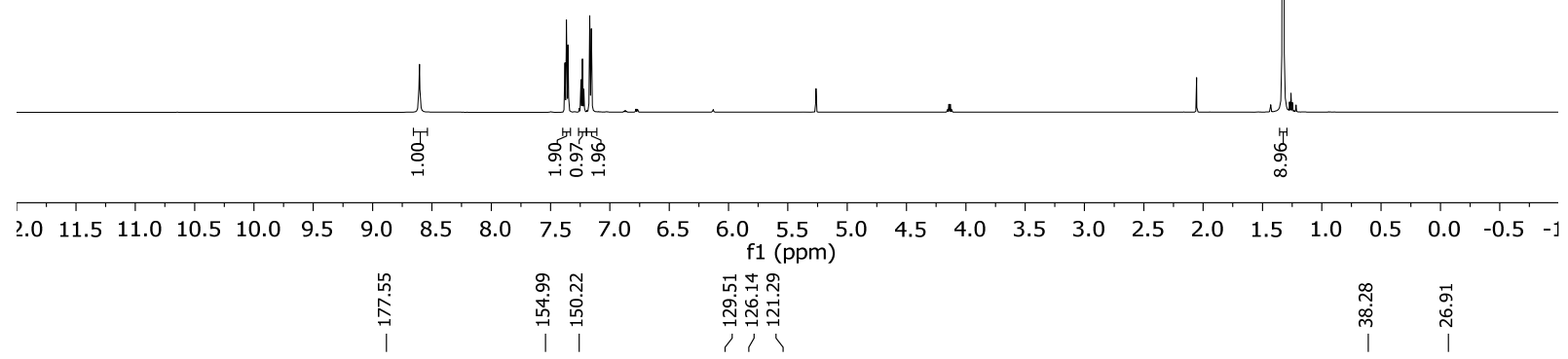

$\gamma_{\mathrm{O}^{-N}}^{\mathrm{O}} \prod_{\mathrm{O}}^{\mathrm{O}}$

2d

${ }^{13} \mathrm{H}$ NMR

$\mathrm{CDCl}_{3}$

$151 \mathrm{MHz}$

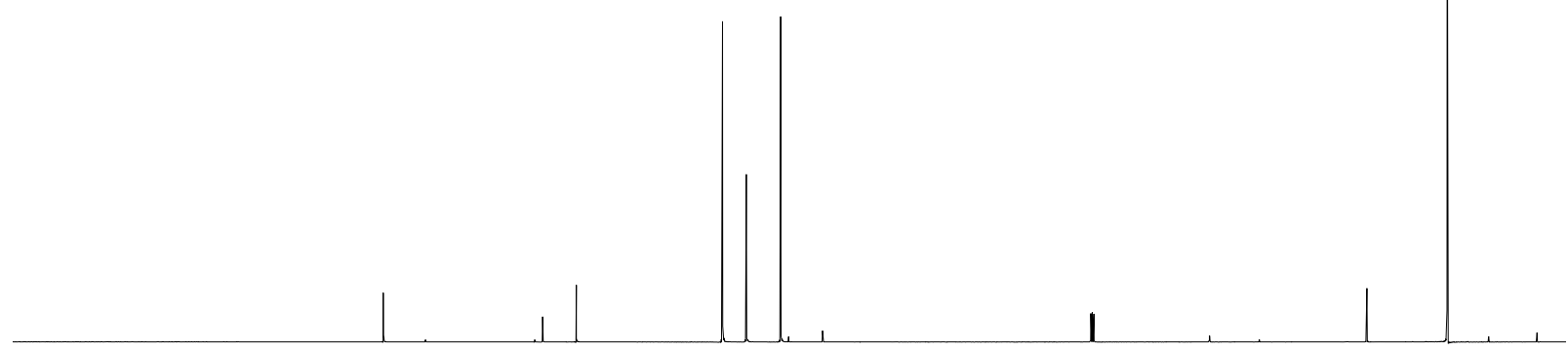

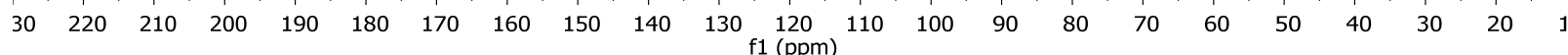




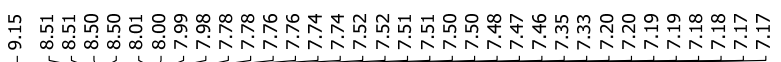

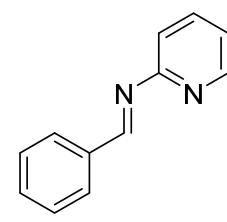

$1 a$

${ }^{1} \mathrm{H}$ NMR

$\mathrm{CDCl}_{3}$

$400 \mathrm{MHz}$
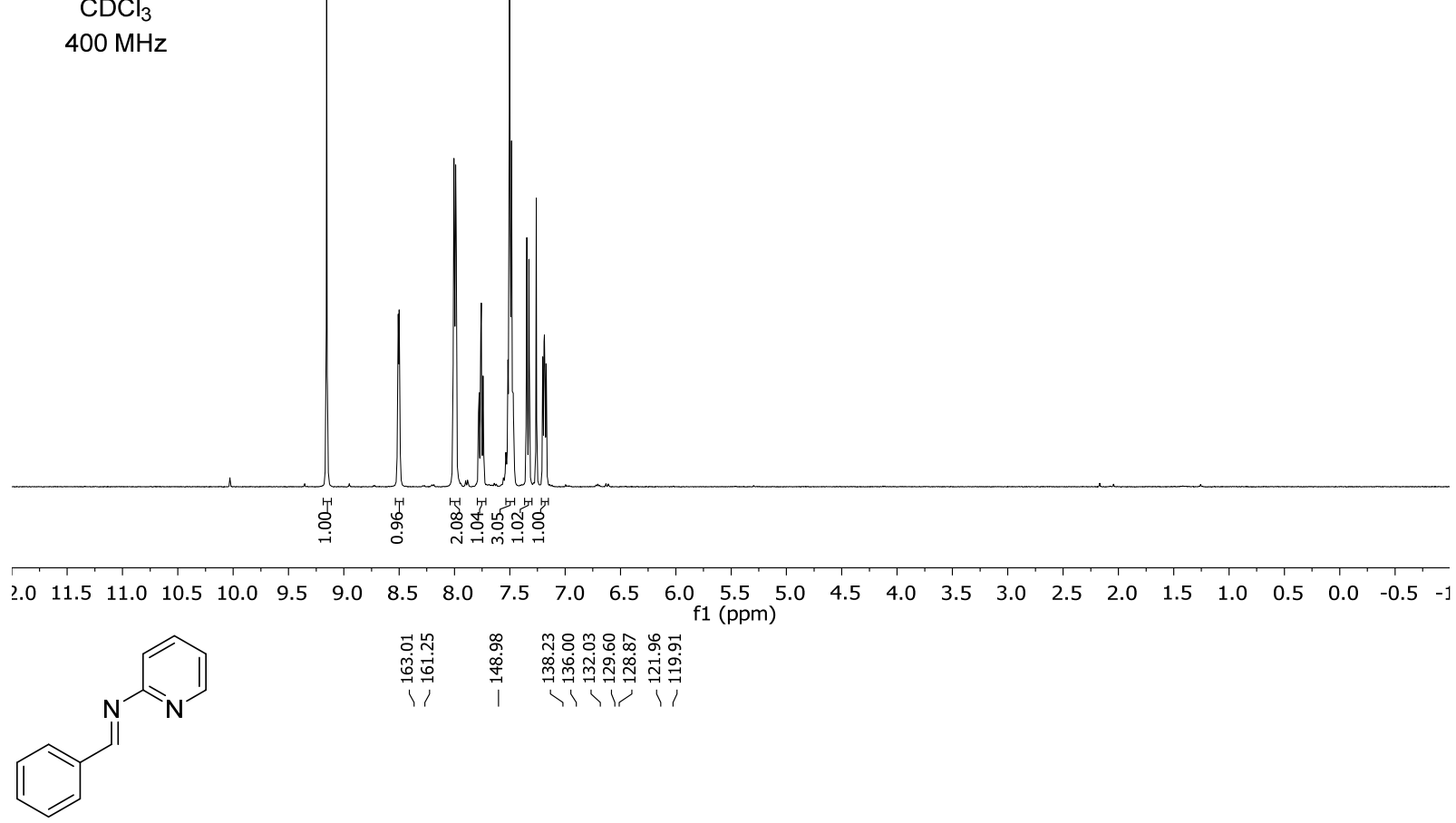

$1 a$

${ }^{13} \mathrm{C}$ NMR

$\mathrm{CDCl}_{3}$

$101 \mathrm{MHz}$

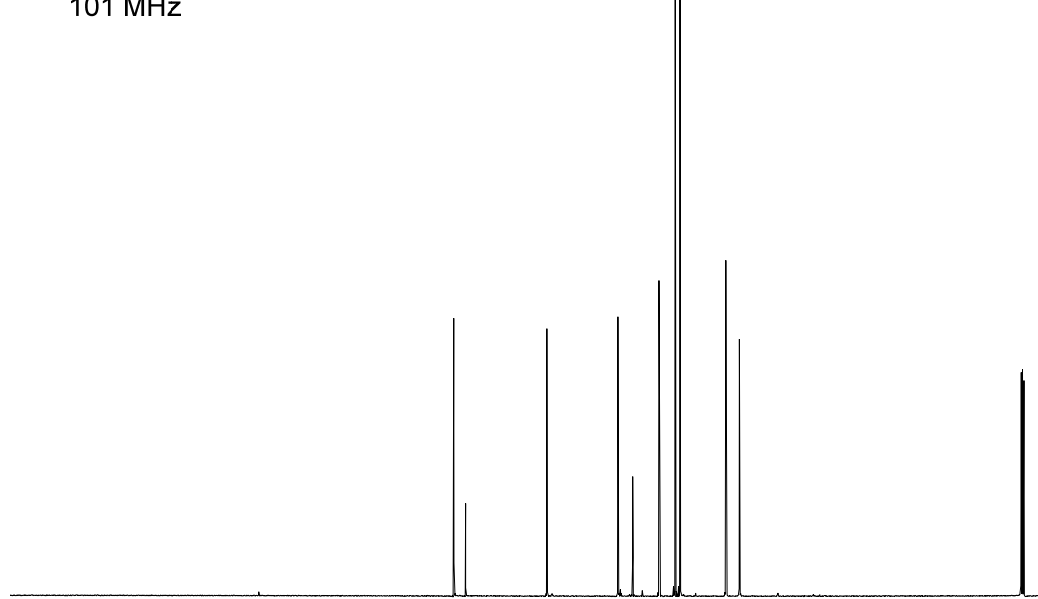

\begin{tabular}{lllllllllllllllllllllllllll}
\hline 30 & 220 & 210 & 200 & 190 & 180 & 170 & 160 & 150 & 140 & 130 & 120 & 110 & 100 & 90 & 80 & 70 & 60 & 50 & 40 & 30 & 20 & 10 & 0 & -
\end{tabular} 

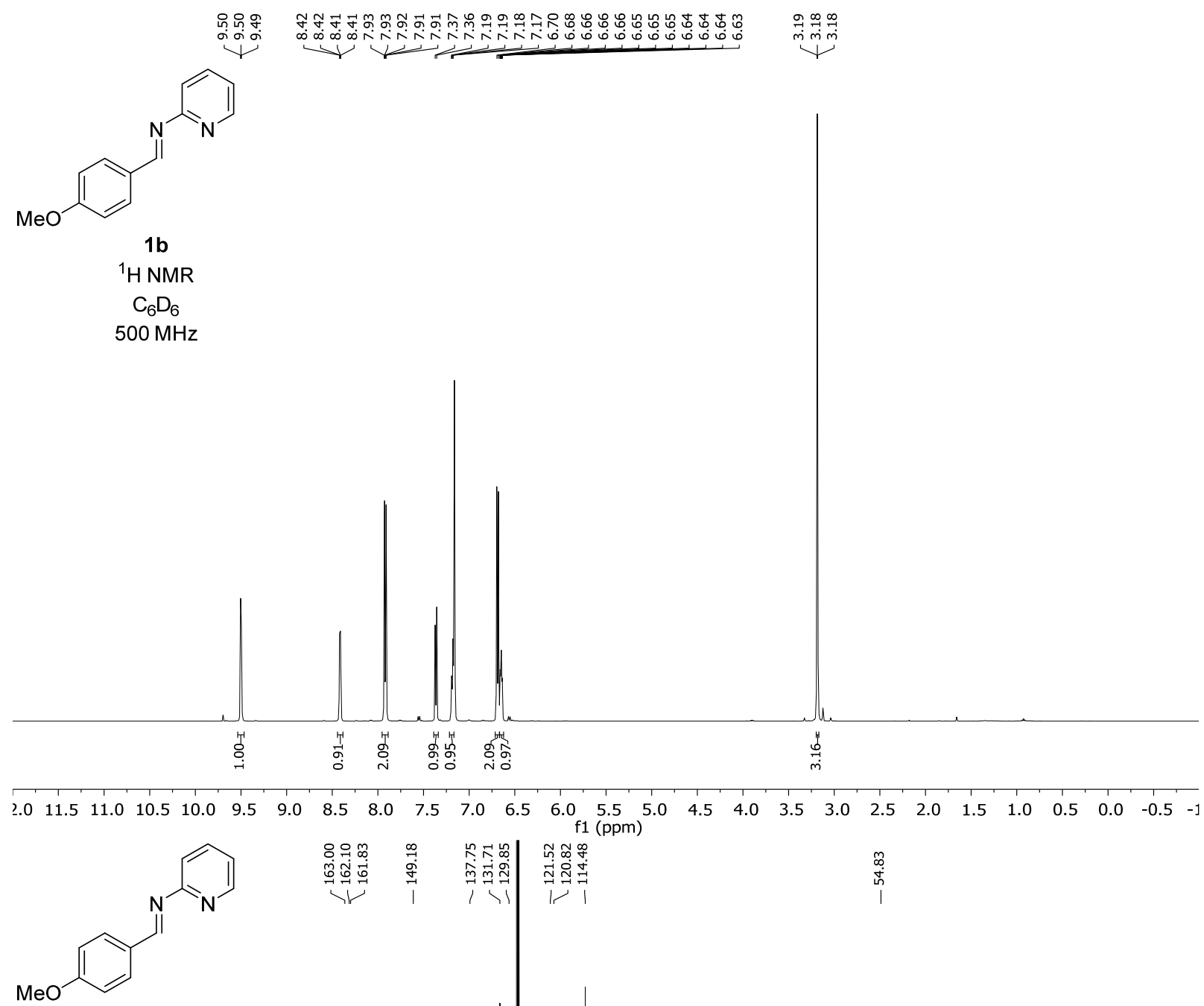

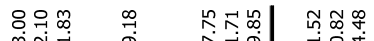

vil

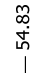

$1 \mathrm{~b}$

${ }^{13} \mathrm{C}$ NMR

$\mathrm{C}_{6} \mathrm{D}_{6}$

$126 \mathrm{MHz}$

$\begin{array}{lllllllllllllllllllllllllllllll}30 & 220 & 210 & 200 & 190 & 180 & 170 & 160 & 150 & 140 & 130 & 120 & 110 & 100 & 90 & 80 & 70 & 60 & 50 & 40 & 30 & 20 & 10 & 0 & -\end{array}$ 


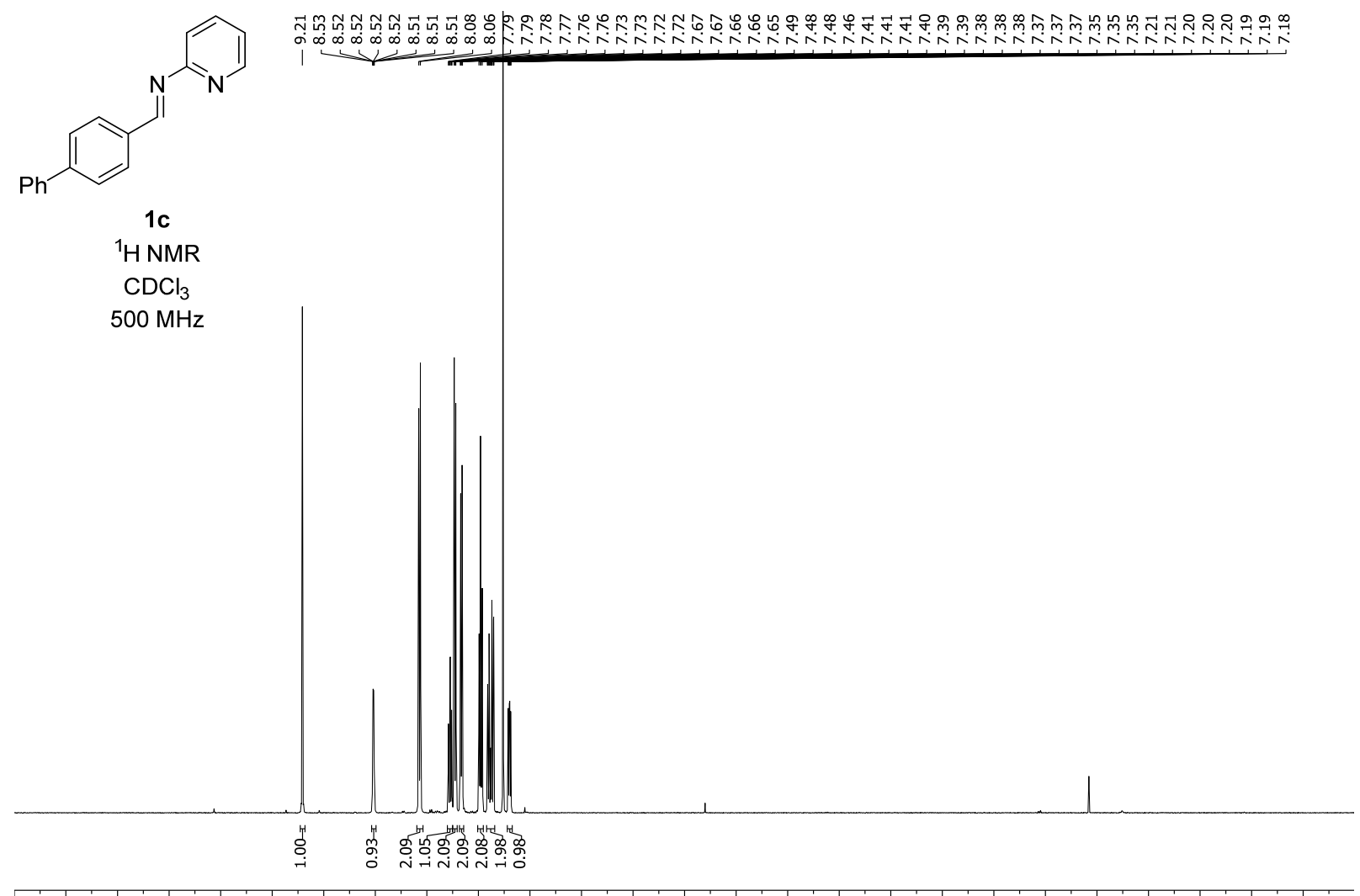

\begin{tabular}{lllllllllllllllllllllllllllllllllllllll}
\hline .0 & 11.5 & 11.0 & 10.5 & 10.0 & 9.5 & 9.0 & 8.5 & 8.0 & 7.5 & 7.0 & 6.5 & 6.0 & 5.5 & 5.0 & 4.5 & 4.0 & 3.5 & 3.0 & 2.5 & 2.0 & 1.5 & 1.0 & 0.5 & 0.0 & -0.5 & -1
\end{tabular}<smiles>C(=N/c1ccc(-c2ccccc2)cc1)\c1ccccn1</smiles>

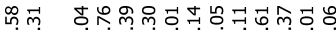

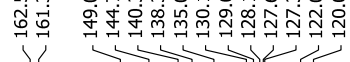

1c

${ }^{13} \mathrm{C}$ NMR

$\mathrm{CDCl}_{3}$

$151 \mathrm{MHz}$

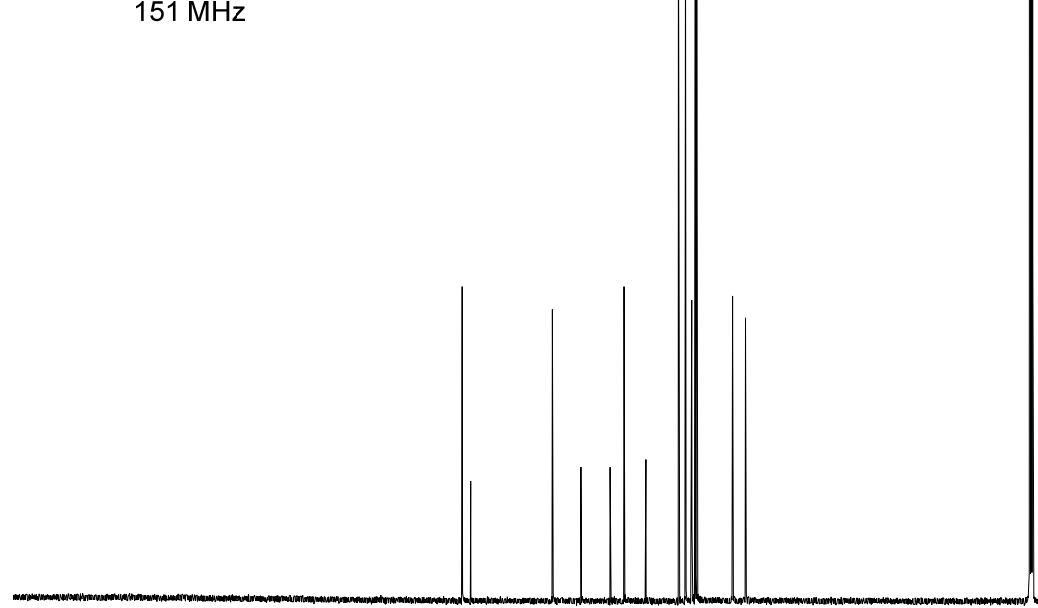

$\begin{array}{lllllllllllllllllllllllllllllllllllllllll}30 & 220 & 210 & 200 & 190 & 180 & 170 & 160 & 150 & 140 & 130 & 120 & 110 & 100 & 90 & 80 & 70 & 60 & 50 & 40 & 30 & 20 & 10 & 0 & -\end{array}$ 


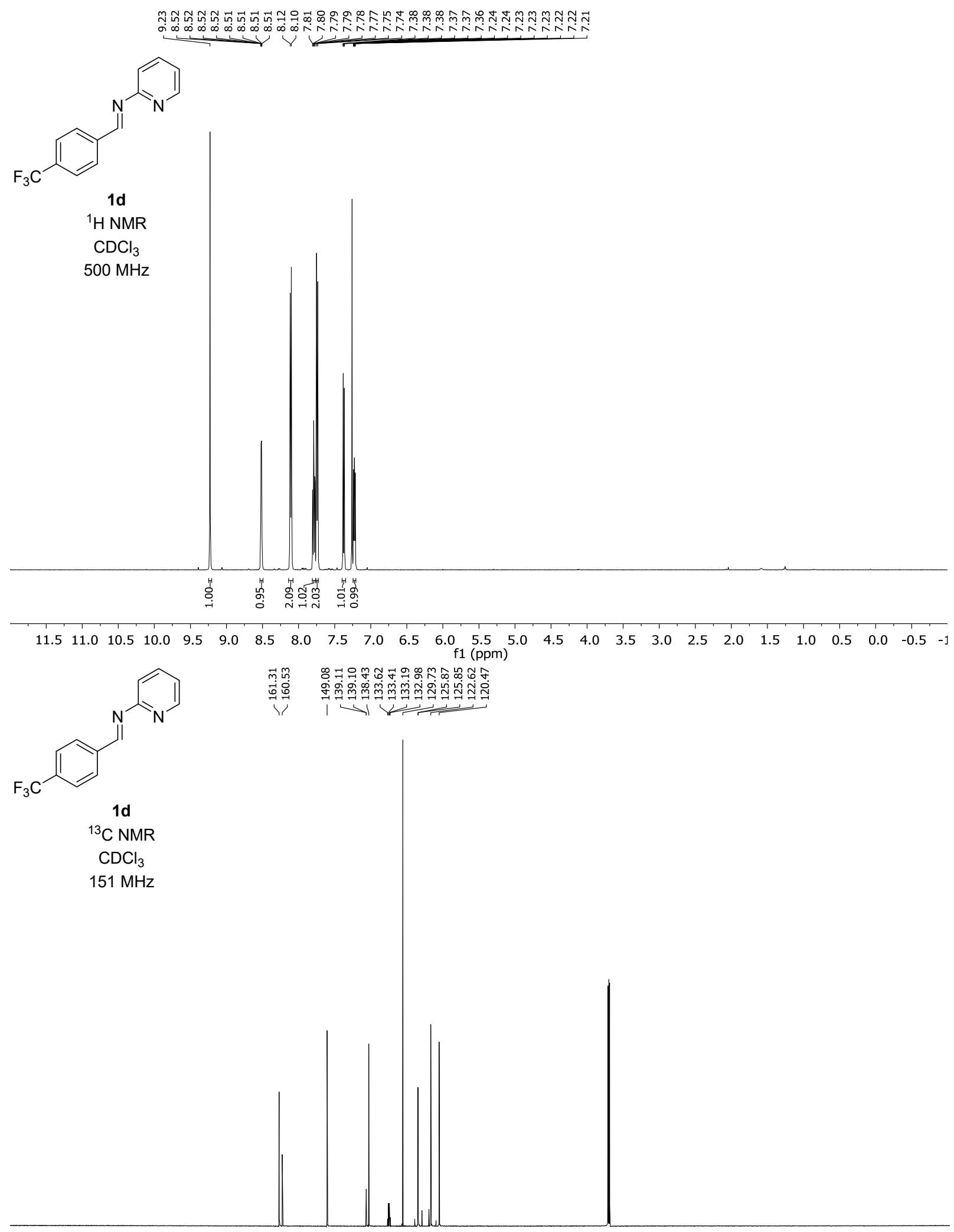

$\begin{array}{llllllllllllllllllllllll}220 & 210 & 200 & 190 & 180 & 170 & 160 & 150 & 140 & 130 & 120 & \begin{array}{l}110 \\ \mathrm{f} 1\end{array}(\mathrm{ppm}) & 100 & 90 & 80 & 70 & 60 & 50 & 40 & 30 & 20 & 10 & 0 & -\end{array}$ 


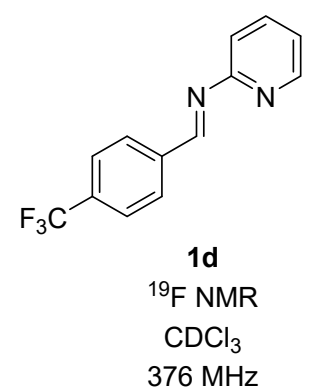

$\begin{array}{llllllllllllllllllllllllllllllllll}30 & 20 & 10 & 0 & -10 & -20 & -30 & -40 & -50 & -60 & -70 & -80 & -90 & -100 & -110 & -120 & -130 & -140 & -150 & -160 & -170 & -180 & -190 & -20 \mathrm{C}\end{array}$ $\mathrm{f} 1(\mathrm{ppm})$ 


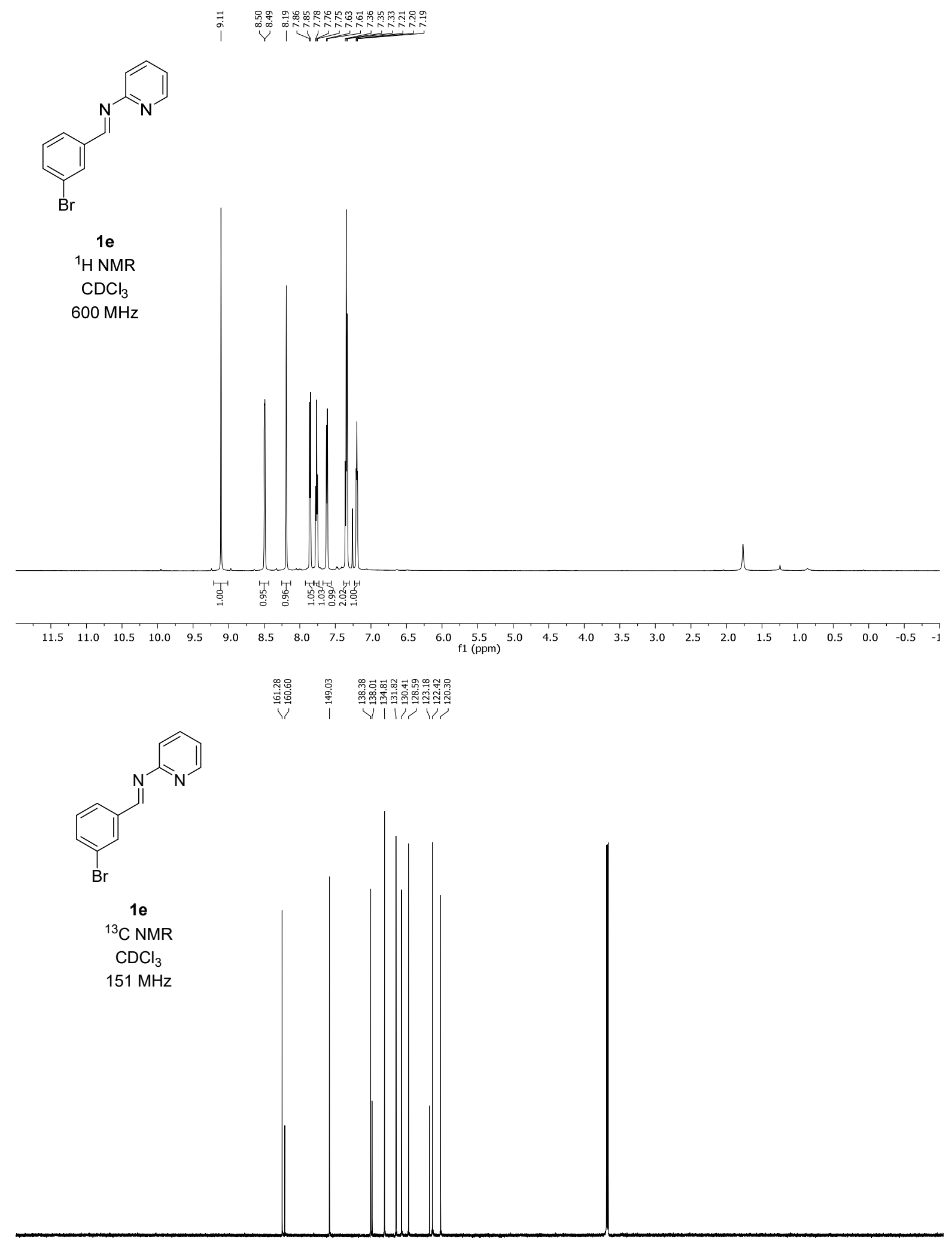

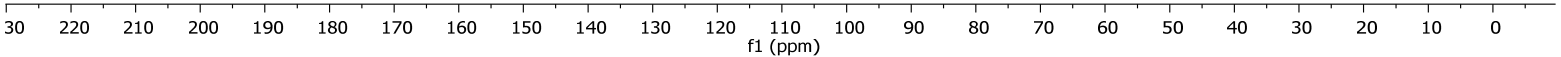




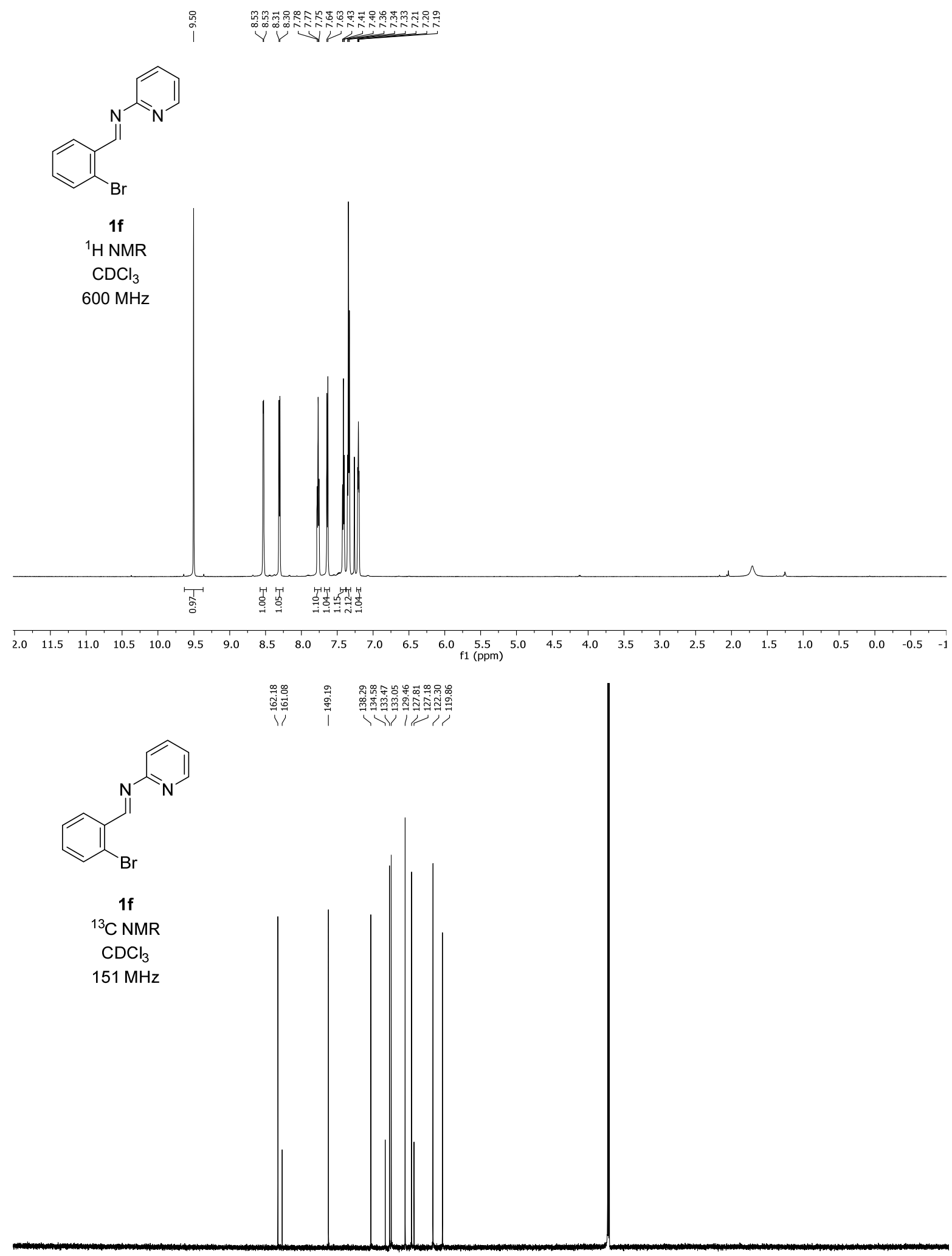

\begin{tabular}{lllllllllllllllllllllllllllll}
\hline 30 & 220 & 210 & 200 & 190 & 180 & 170 & 160 & 150 & 140 & 130 & 120 & 110 & 100 & 90 & 80 & 70 & 60 & 50 & 40 & 30 & 20 & 10 & 0 & -1
\end{tabular} 

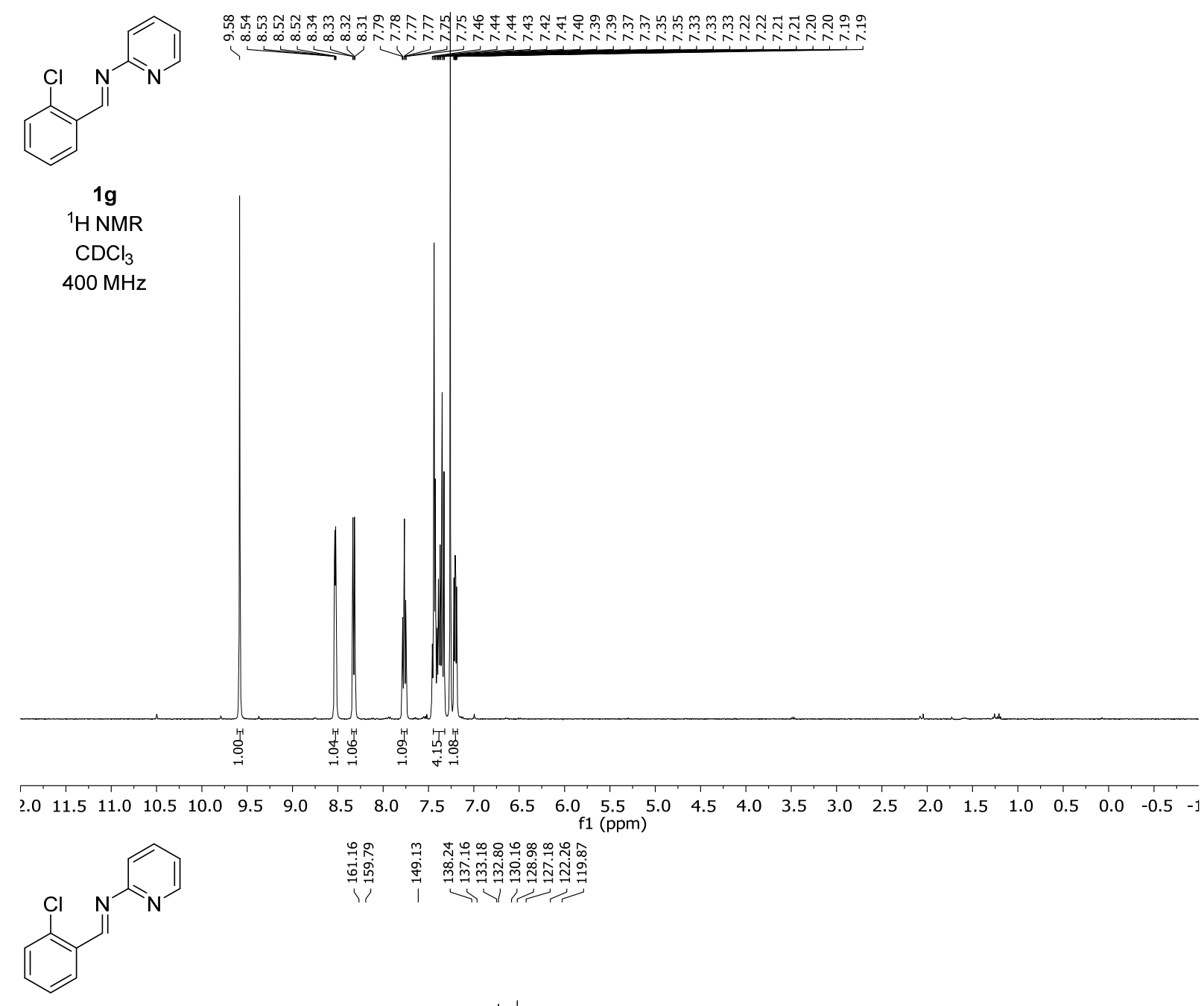

$1 \mathrm{~g}$

${ }^{13} \mathrm{C}$ NMR

$\mathrm{CDCl}_{3}$

$151 \mathrm{MHz}$

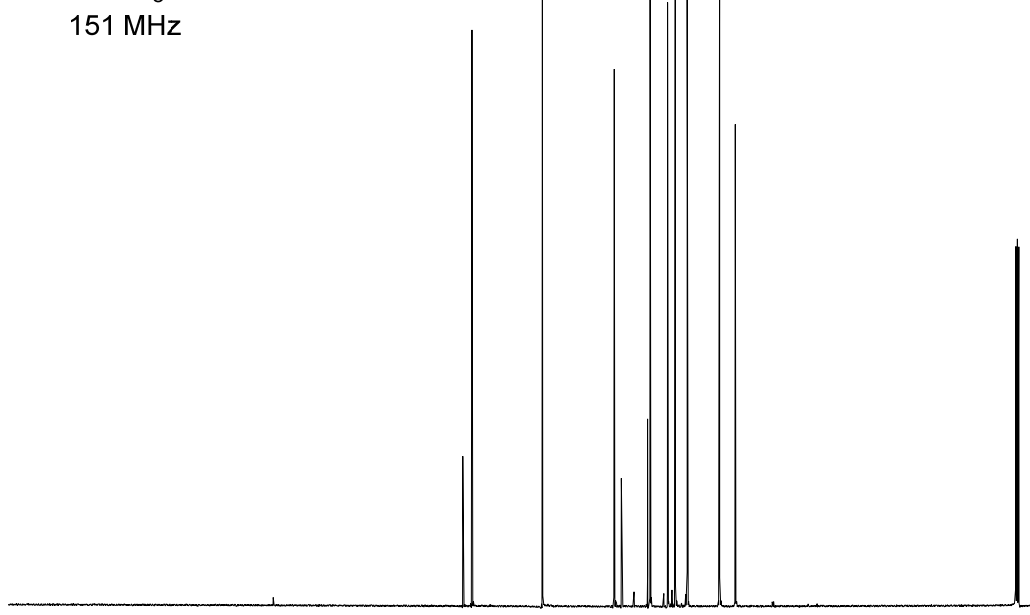

$\begin{array}{lllllllllllllllllllllllll}30 & 220 & 210 & 200 & 190 & 180 & 170 & 160 & 150 & 140 & 130 & 120 & 110 & 100 & 90 & 80 & 70 & 60 & 50 & 40 & 30 & 20 & 10 & 0 & -\end{array}$ 


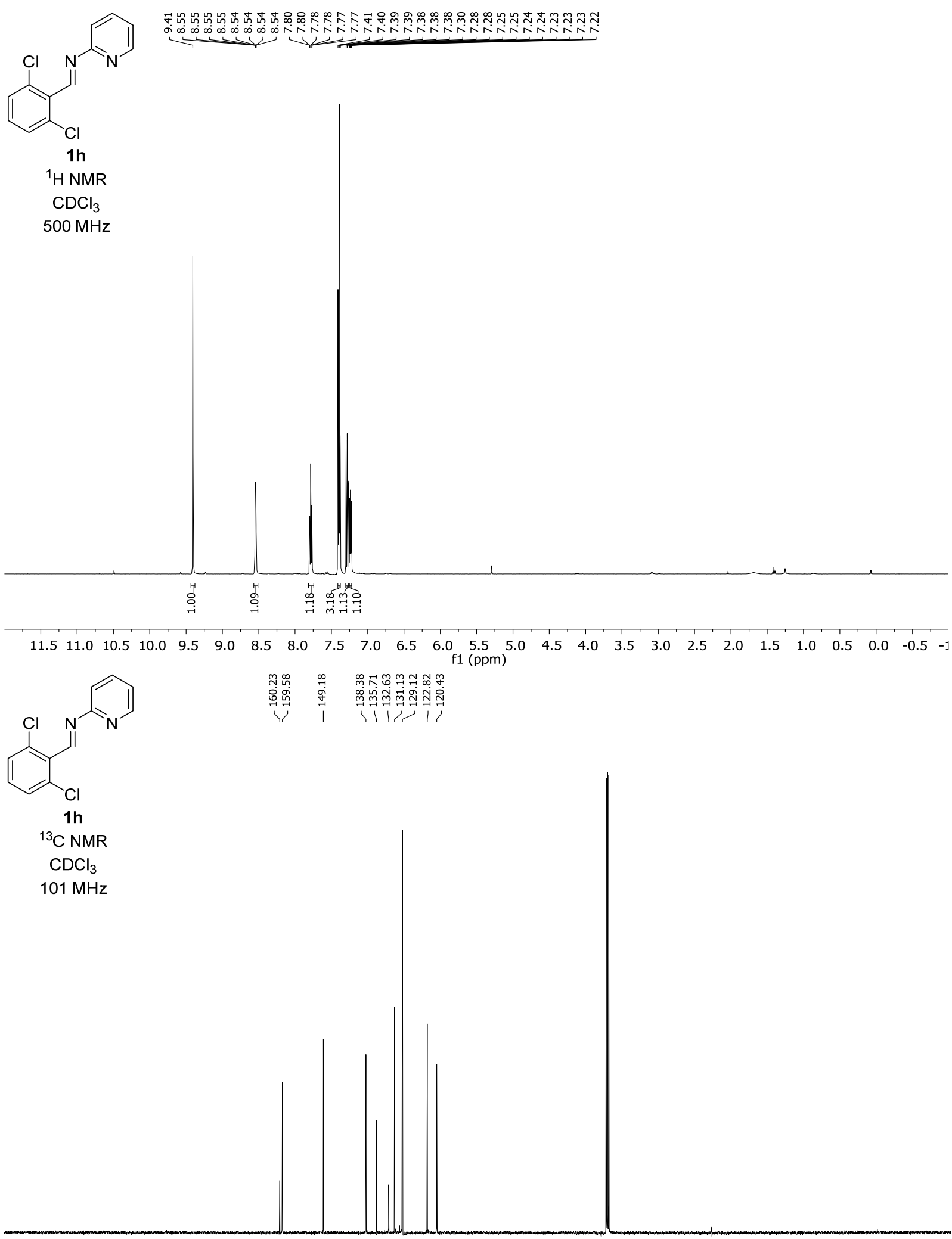

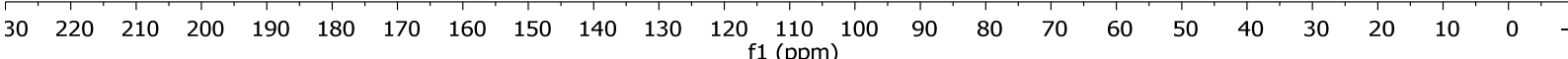




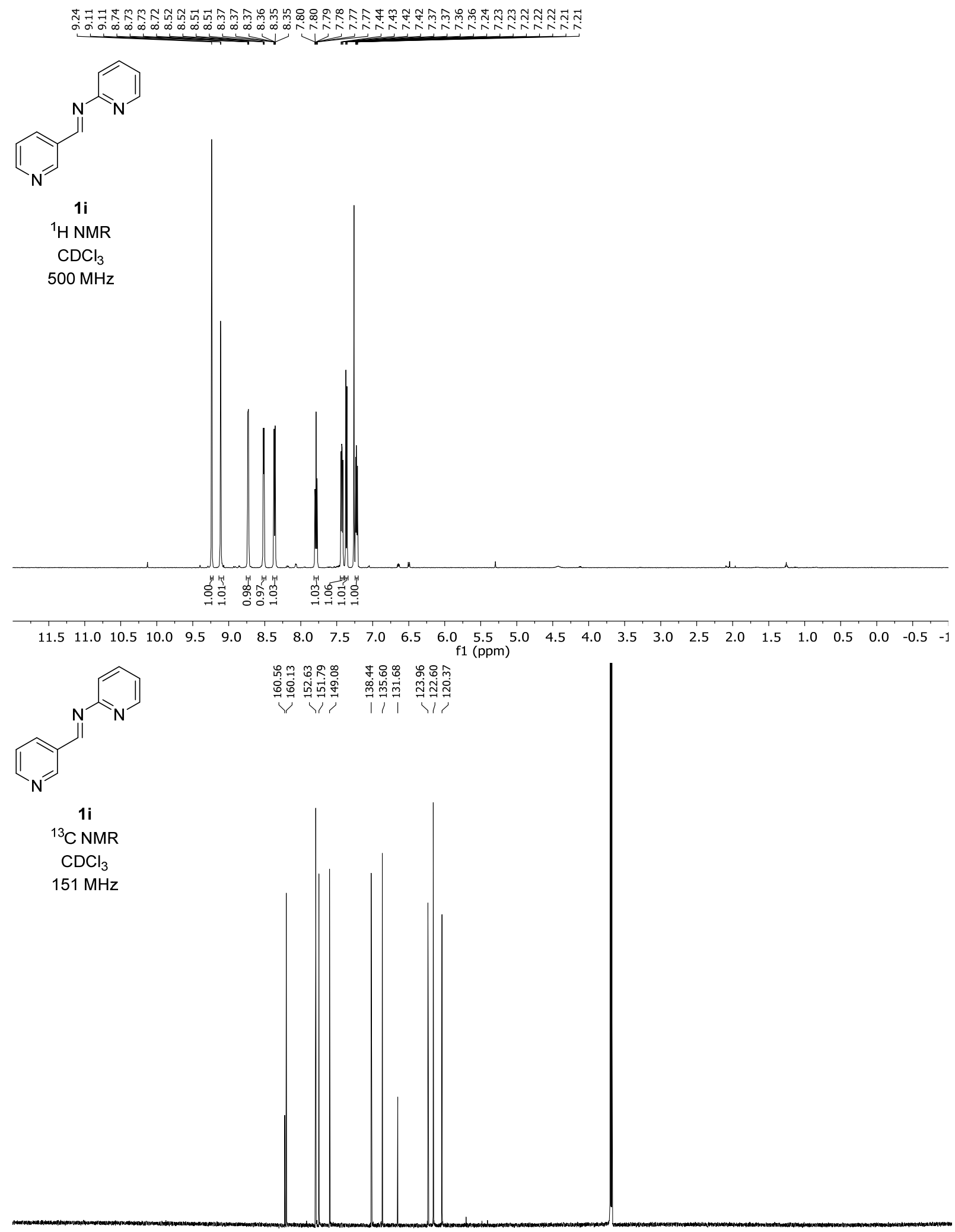

$\begin{array}{lllllllllllllllllllllllllllllll}30 & 220 & 210 & 200 & 190 & 180 & 170 & 160 & 150 & 140 & 130 & 120 & 110 & 100 & 90 & 80 & 70 & 60 & 50 & 40 & 30 & 20 & 10 & 0 & -\end{array}$ 


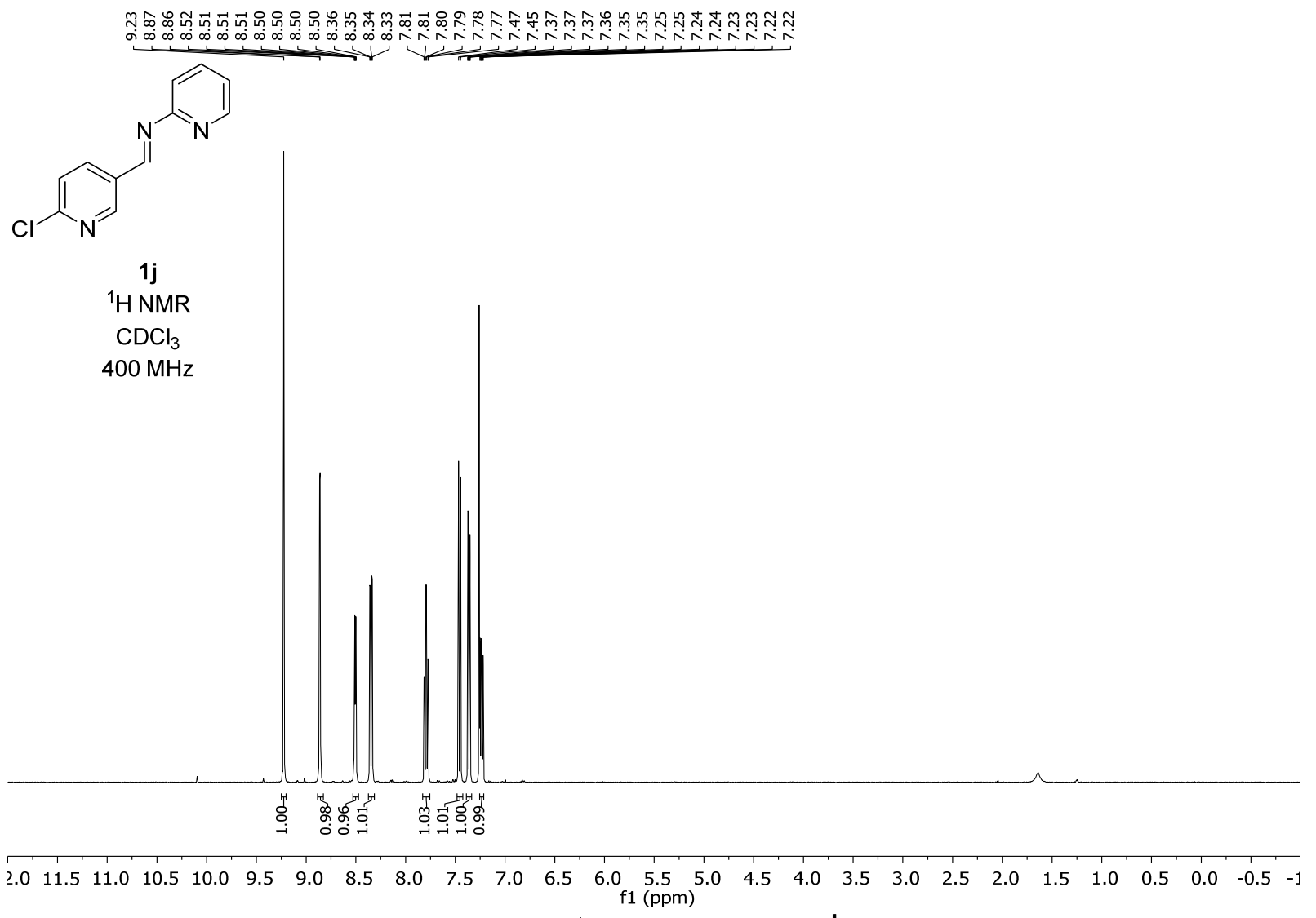<smiles>Clc1ccc(C=Nc2ccccn2)cn1</smiles>

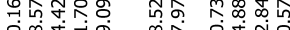

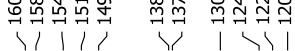

$1 \mathrm{j}$

${ }^{13} \mathrm{C}$ NMR $\mathrm{CDCl}_{3}$ $151 \mathrm{MHz}$

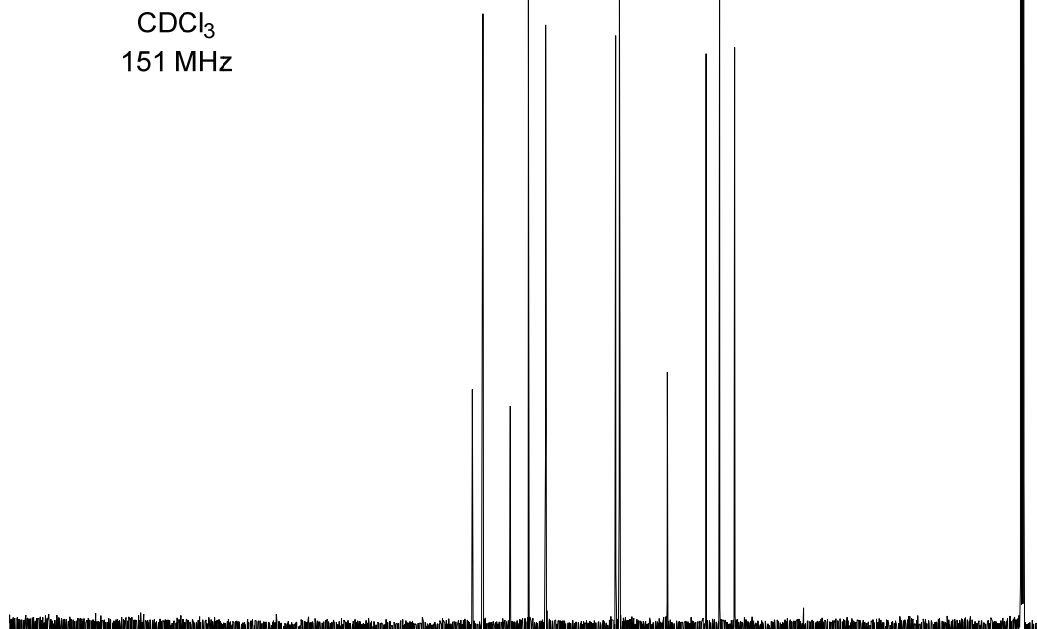




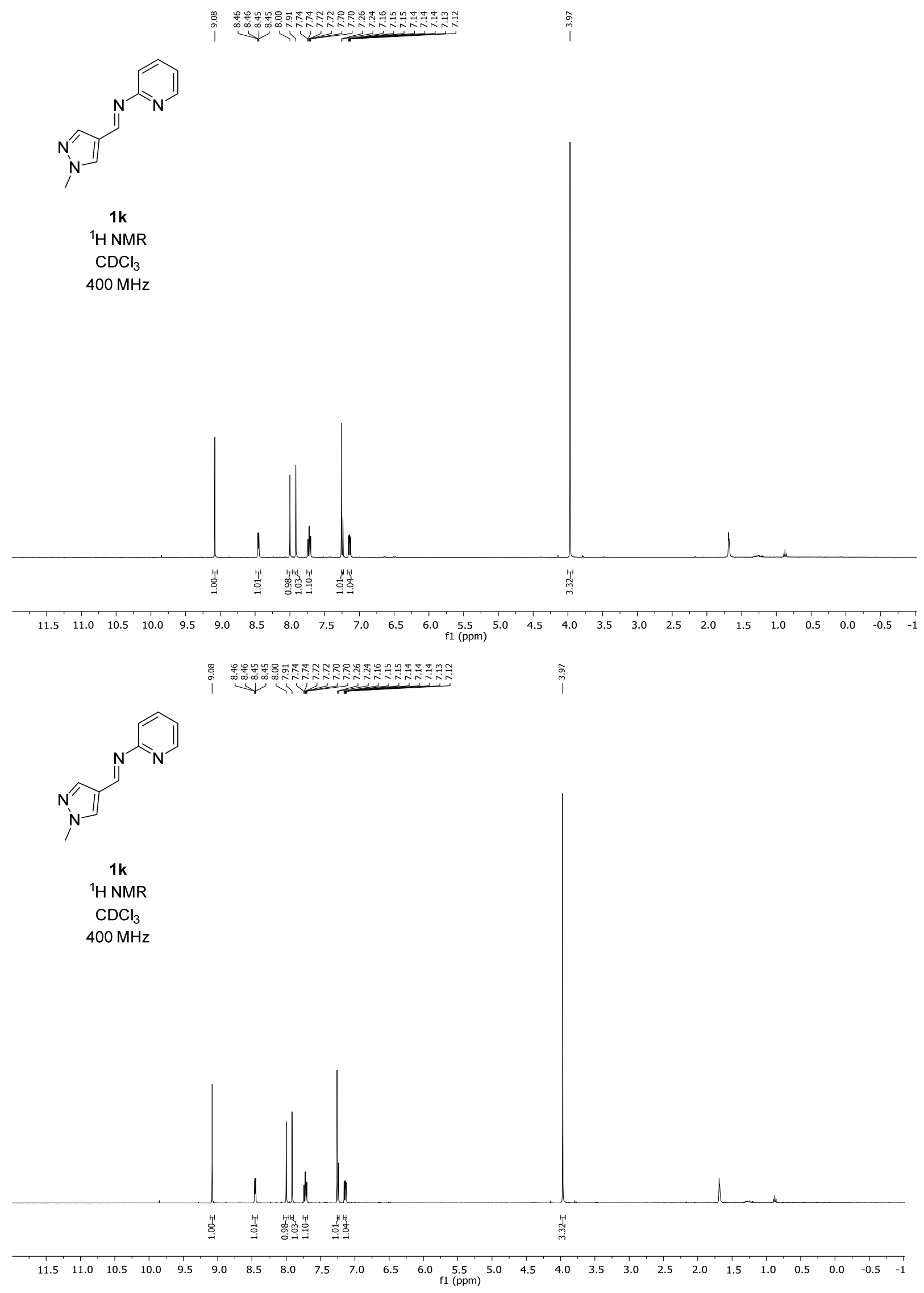




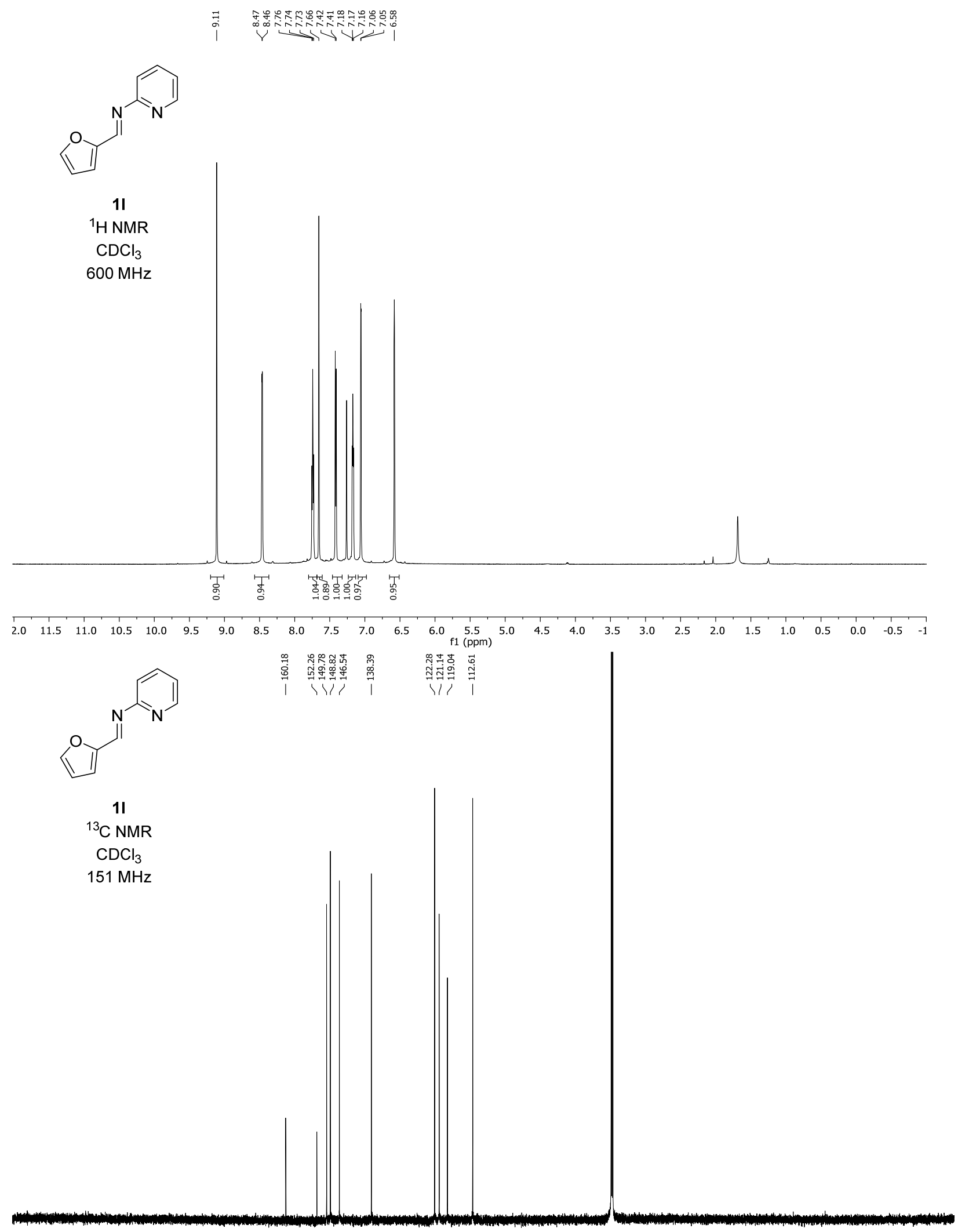

$\begin{array}{llllllllllllllllllllllll}220 & 210 & 200 & 190 & 180 & 170 & 160 & 150 & 140 & 130 & 120 & \begin{array}{l}110 \\ \mathrm{f} 1(\mathrm{ppm})\end{array} & 100 & 90 & 80 & 70 & 60 & 50 & 40 & 30 & 20 & 10 & 0 & \end{array}$ 


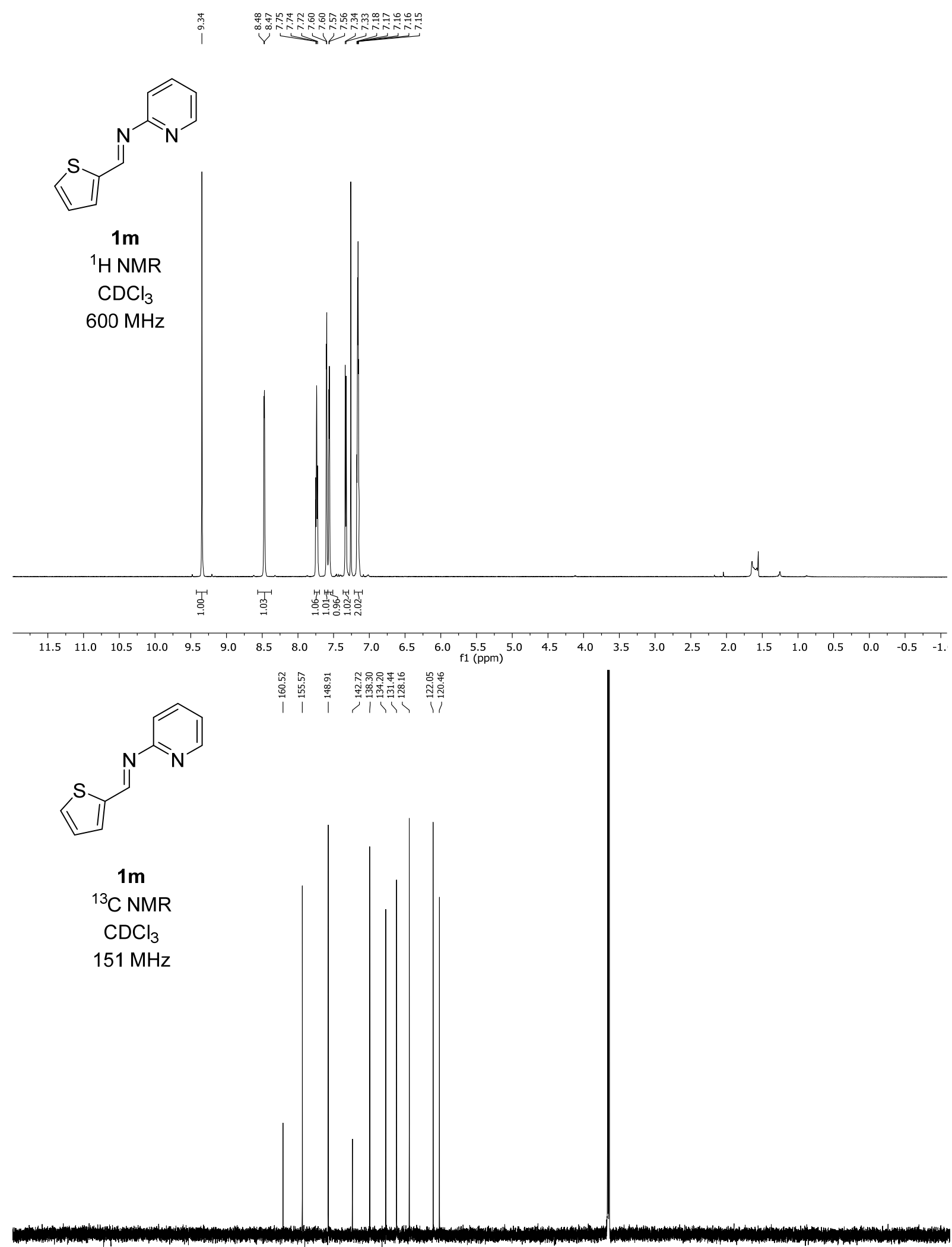

$\begin{array}{lllllllllllllllllllllllll} & 1 \\ 220 & 210 & 200 & 190 & 180 & 170 & 160 & 150 & 140 & 130 & 120 & 110 & 100 & 90 & 80 & 70 & 60 & 50 & 40 & 30 & 20 & 10 & 0 & -1\end{array}$ 


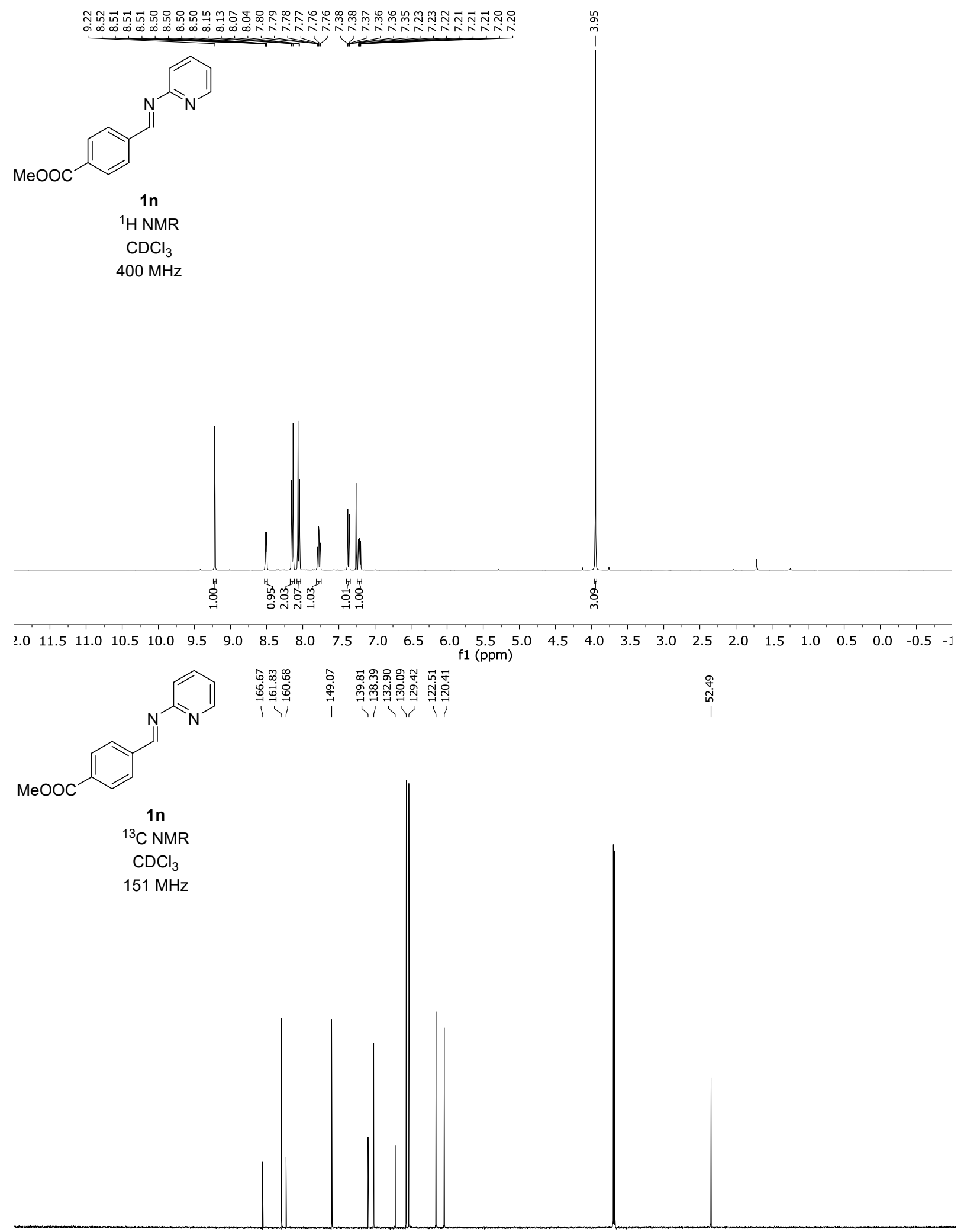

\begin{tabular}{lllllllllllllllllllllllllllll}
\hline 30 & 220 & 210 & 200 & 190 & 180 & 170 & 160 & 150 & 140 & 130 & 120 & $\begin{array}{l}110 \\
\mathrm{f} 1\end{array}(\mathrm{ppm})$ & 100 & 90 & 80 & 70 & 60 & 50 & 40 & 30 & 20 & 10 & 0 & -
\end{tabular} 


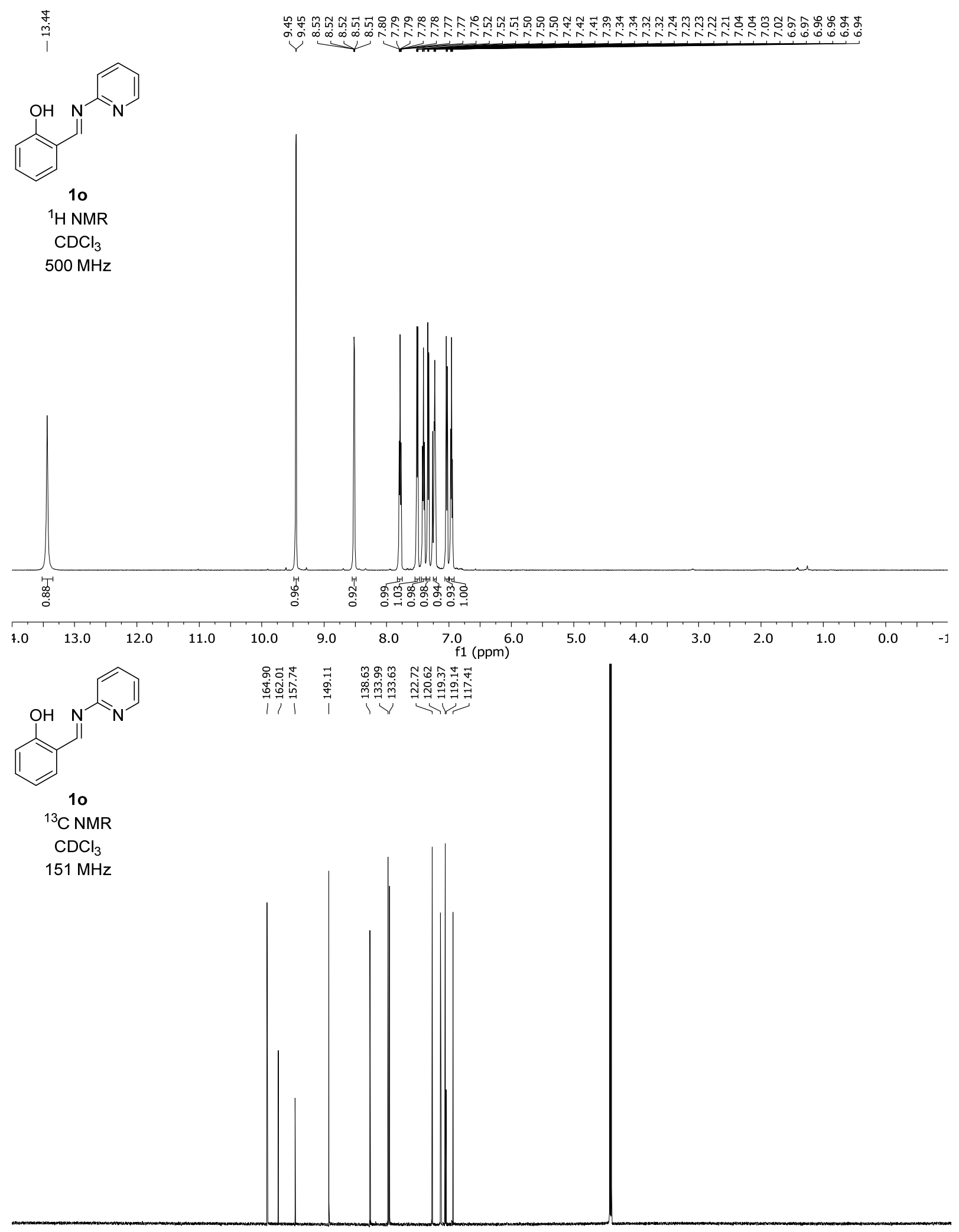

$\begin{array}{llllllllllllllllllllllllllllll}30 & 220 & 210 & 200 & 190 & 180 & 170 & 160 & 150 & 140 & 130 & 120 & \begin{array}{l}110 \\ \mathrm{f} 1\end{array}(\mathrm{ppm}) & 100 & 90 & 80 & 70 & 60 & 50 & 40 & 30 & 20 & 10 & 0 & -\end{array}$ 


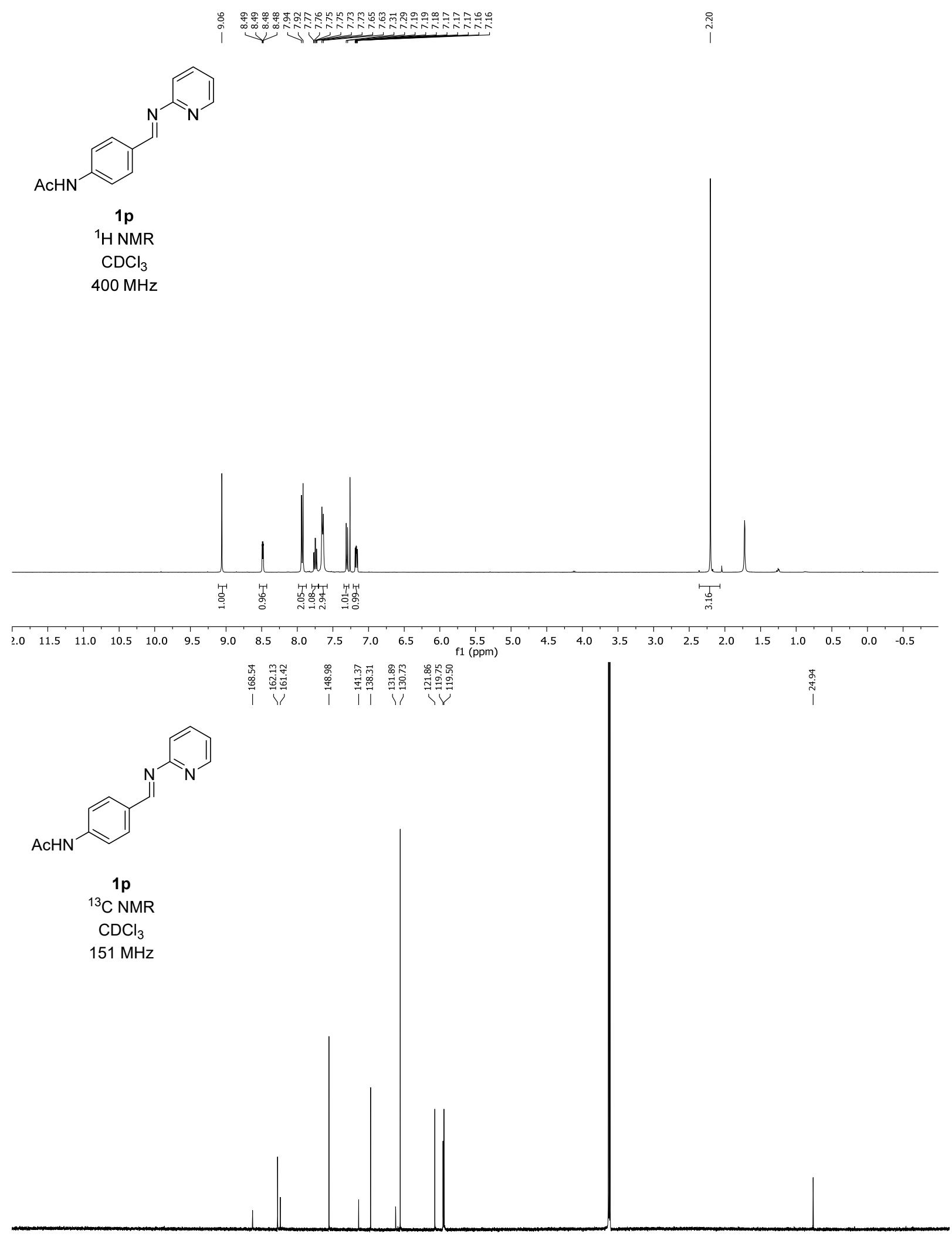

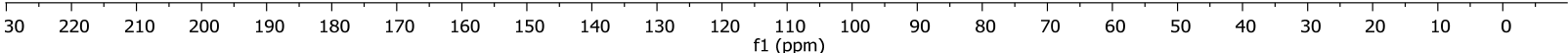



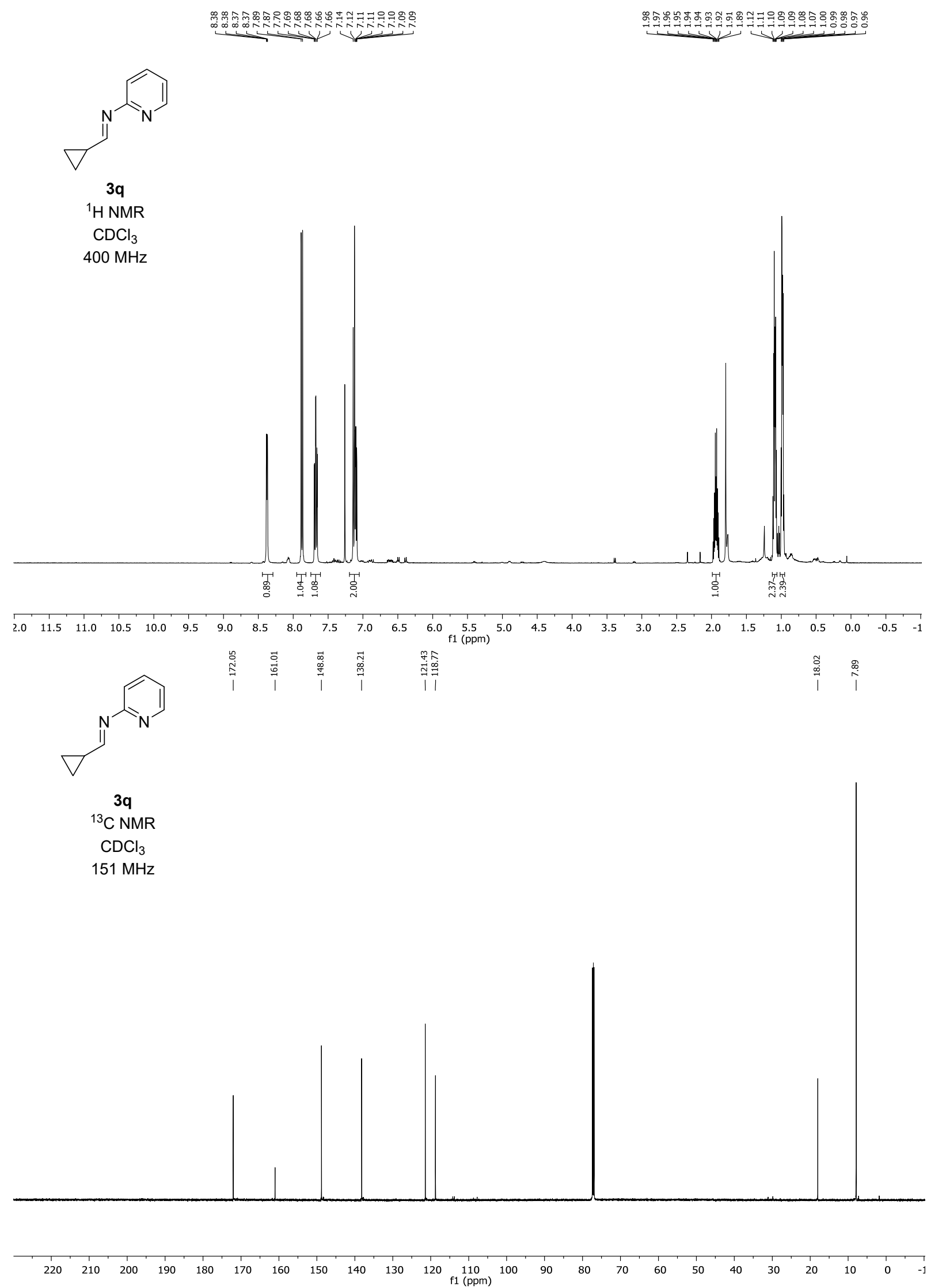

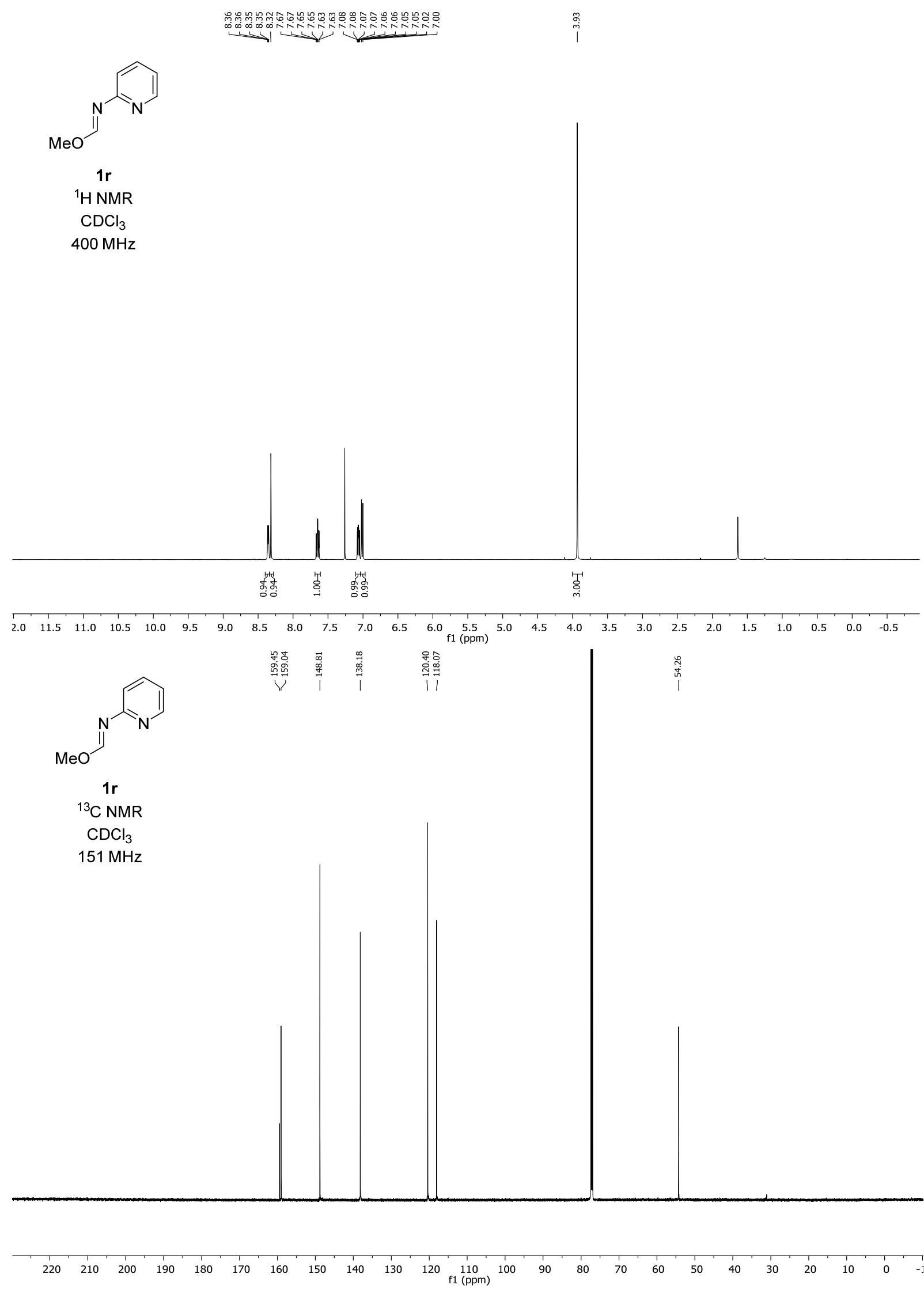


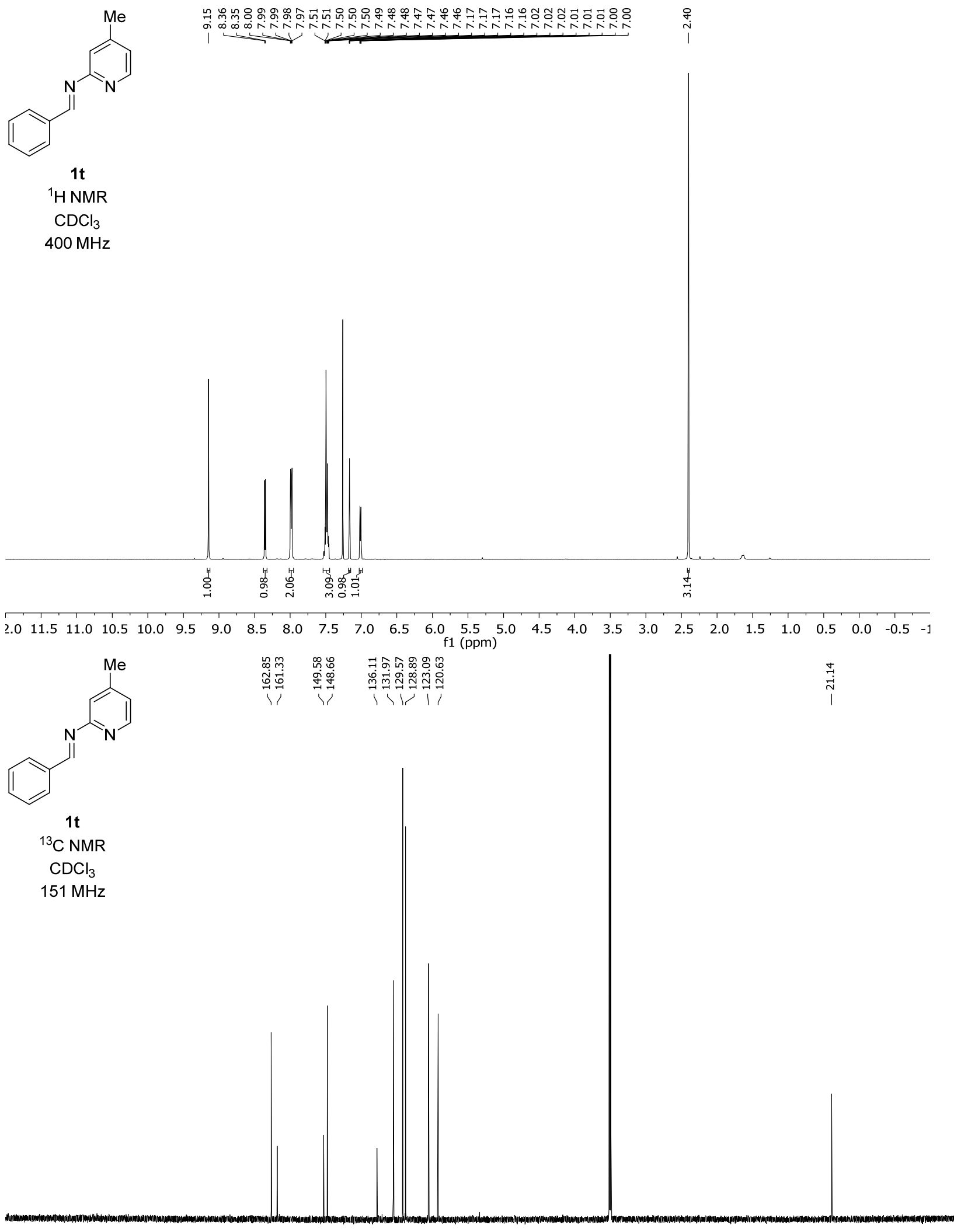

$\begin{array}{llllllllllllllllllllllllllllllllll}30 & 220 & 210 & 200 & 190 & 180 & 170 & 160 & 150 & 140 & 130 & 120 & 110 & 100 & 90 & 80 & 70 & 60 & 50 & 40 & 30 & 20 & 10 & 0 & -\end{array}$ 

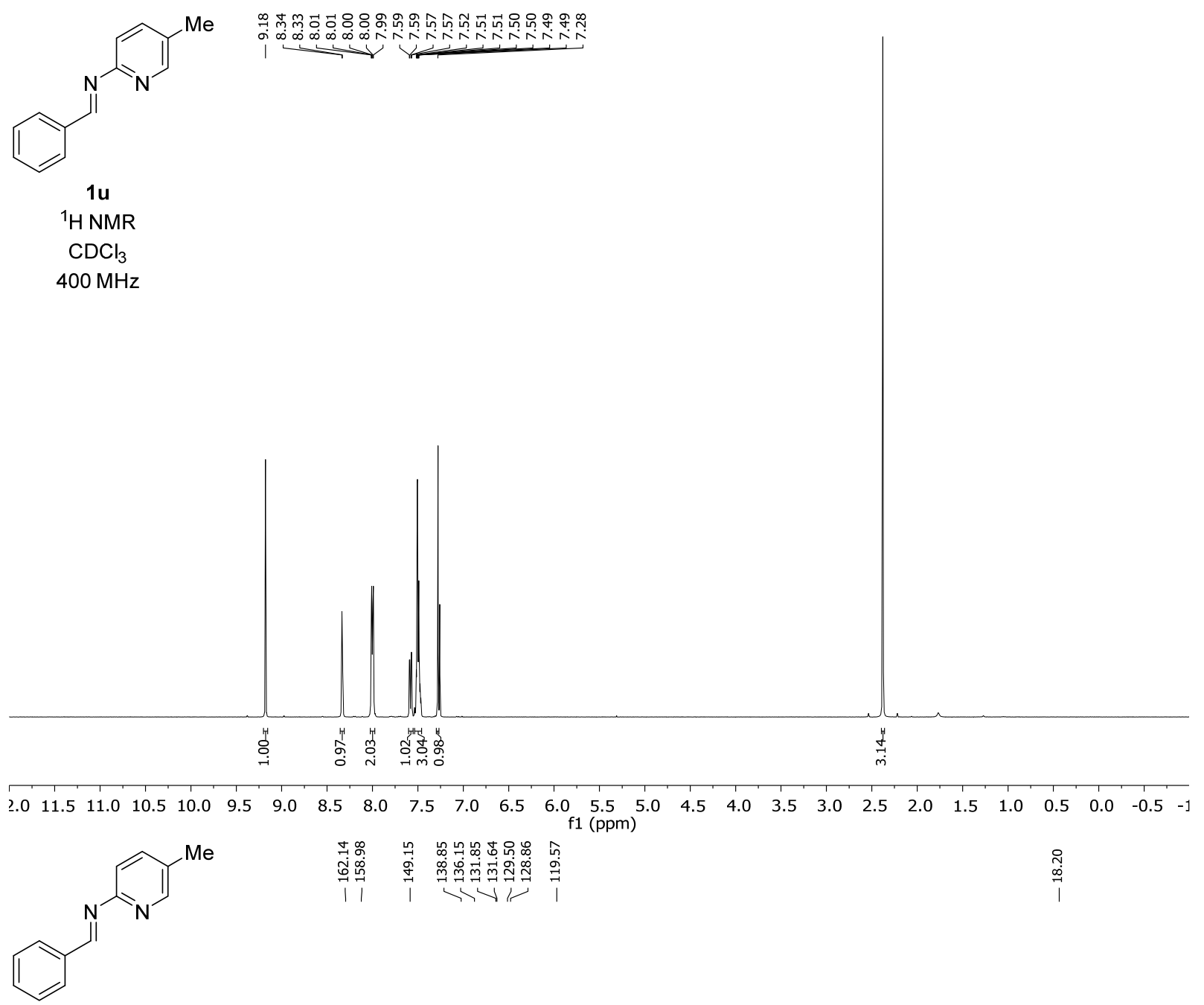

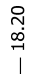

$1 \mathrm{u}$

${ }^{13} \mathrm{C}$ NMR $\mathrm{CDCl}_{3}$

$151 \mathrm{MHz}$

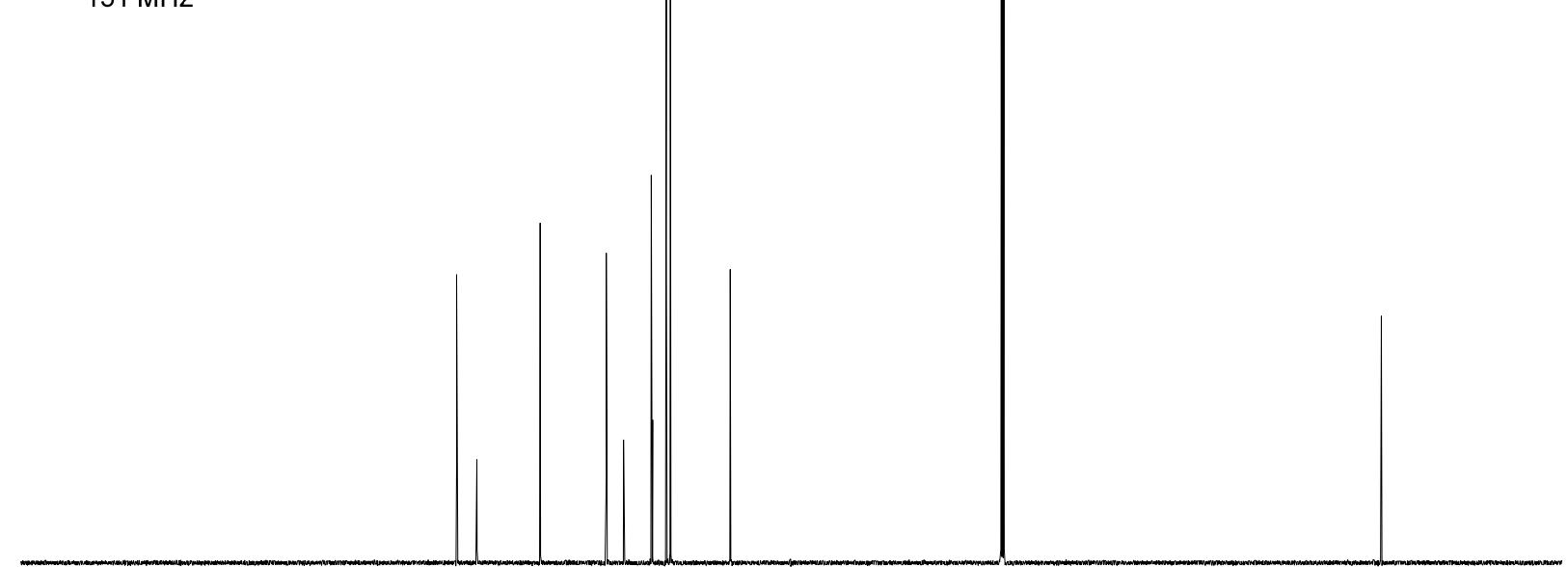

30

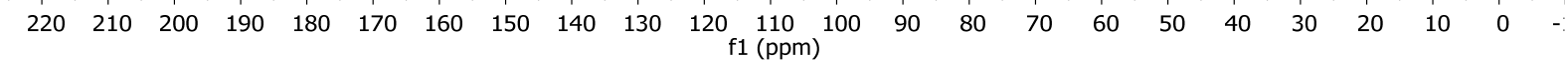



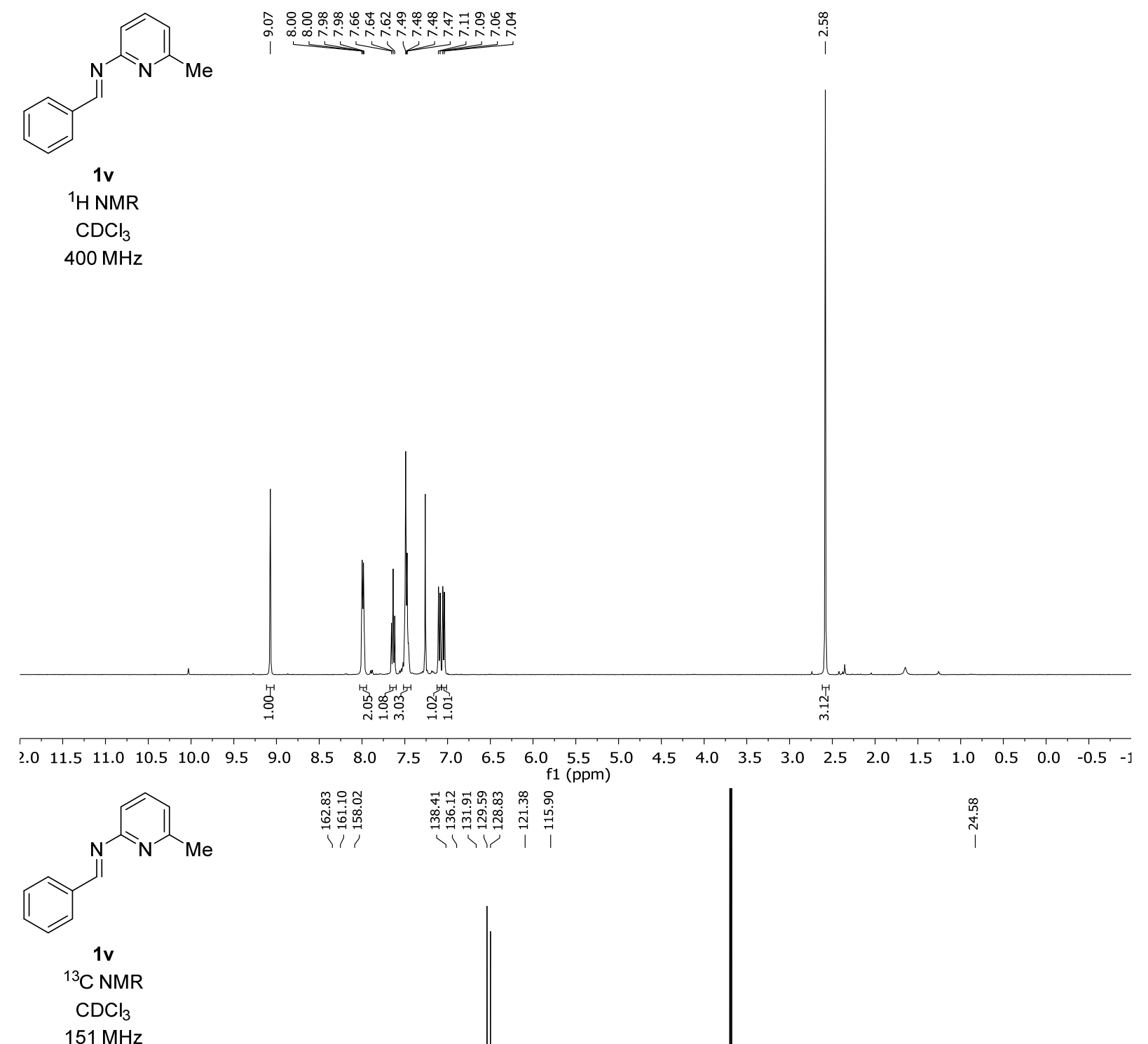

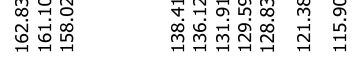

।1 पर। ।

$\stackrel{\substack{n \\ \stackrel{\leftrightarrow}{\sim}}}{\mid}$

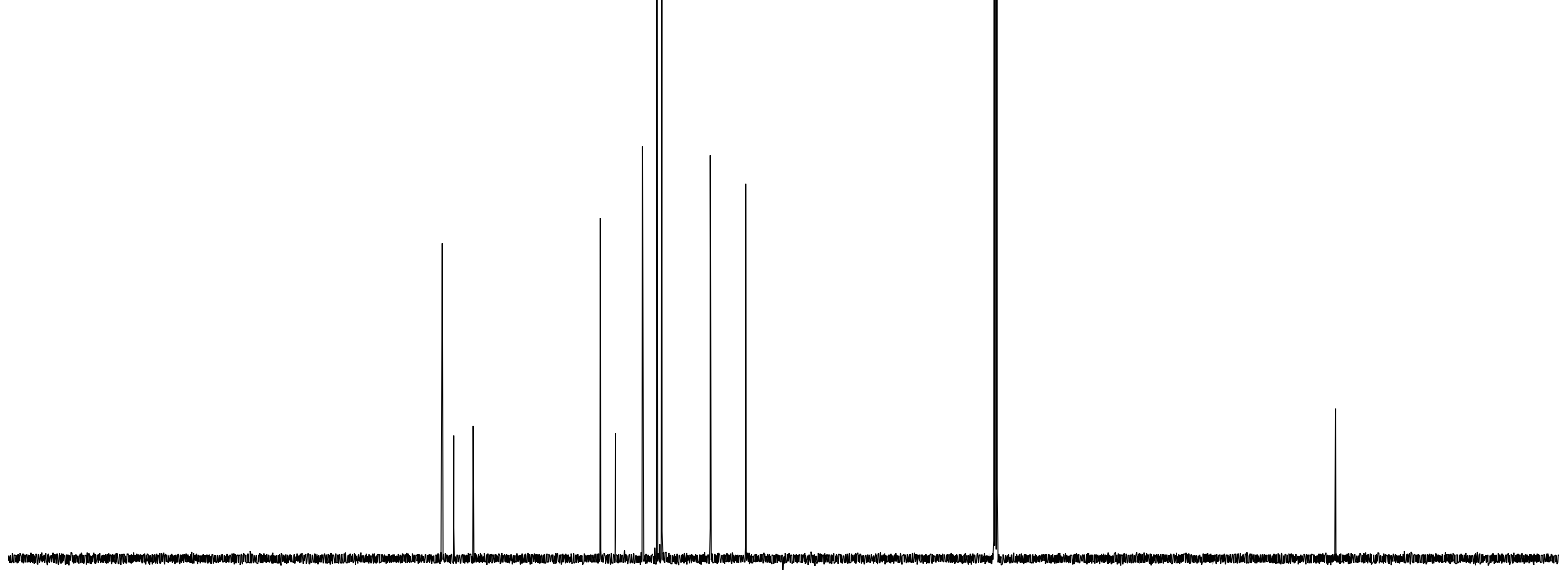

\begin{tabular}{llllllllllllllllllllllllll}
\hline 30 & 220 & 210 & 200 & 190 & 180 & 170 & 160 & 150 & 140 & 130 & 120 & $\begin{array}{l}110 \\
\mathrm{f} 1\end{array}(\mathrm{ppm})$ & 100 & 90 & 80 & 70 & 60 & 50 & 40 & 30 & 20 & 10 & 0 & -
\end{tabular} 


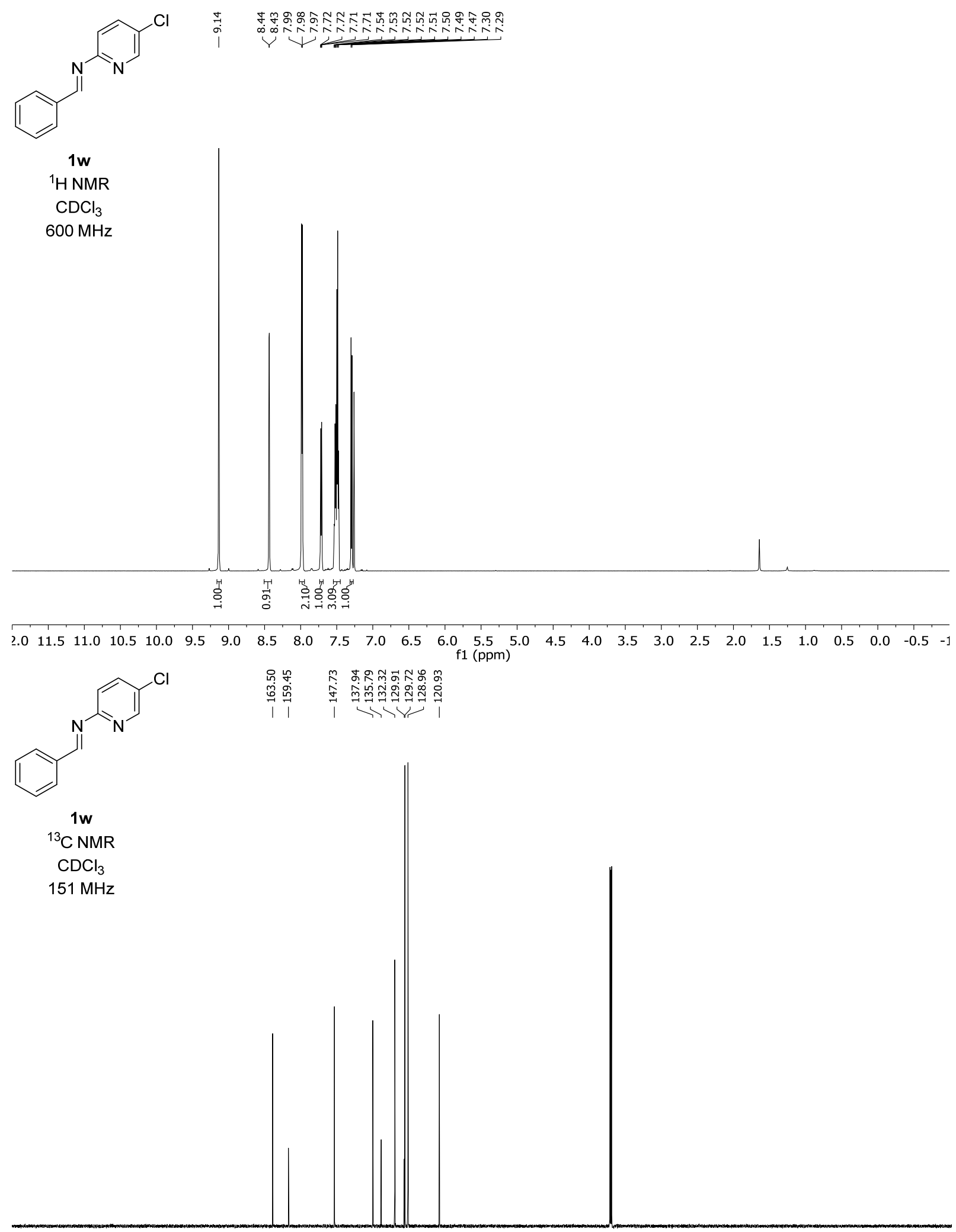

\begin{tabular}{lllllllllllllllllllllllllll}
\hline 30 & 220 & 210 & 200 & 190 & 180 & 170 & 160 & 150 & 140 & 130 & 120 & 110 & 100 & 90 & 80 & 70 & 60 & 50 & 40 & 30 & 20 & 10 & 0 & -
\end{tabular} 

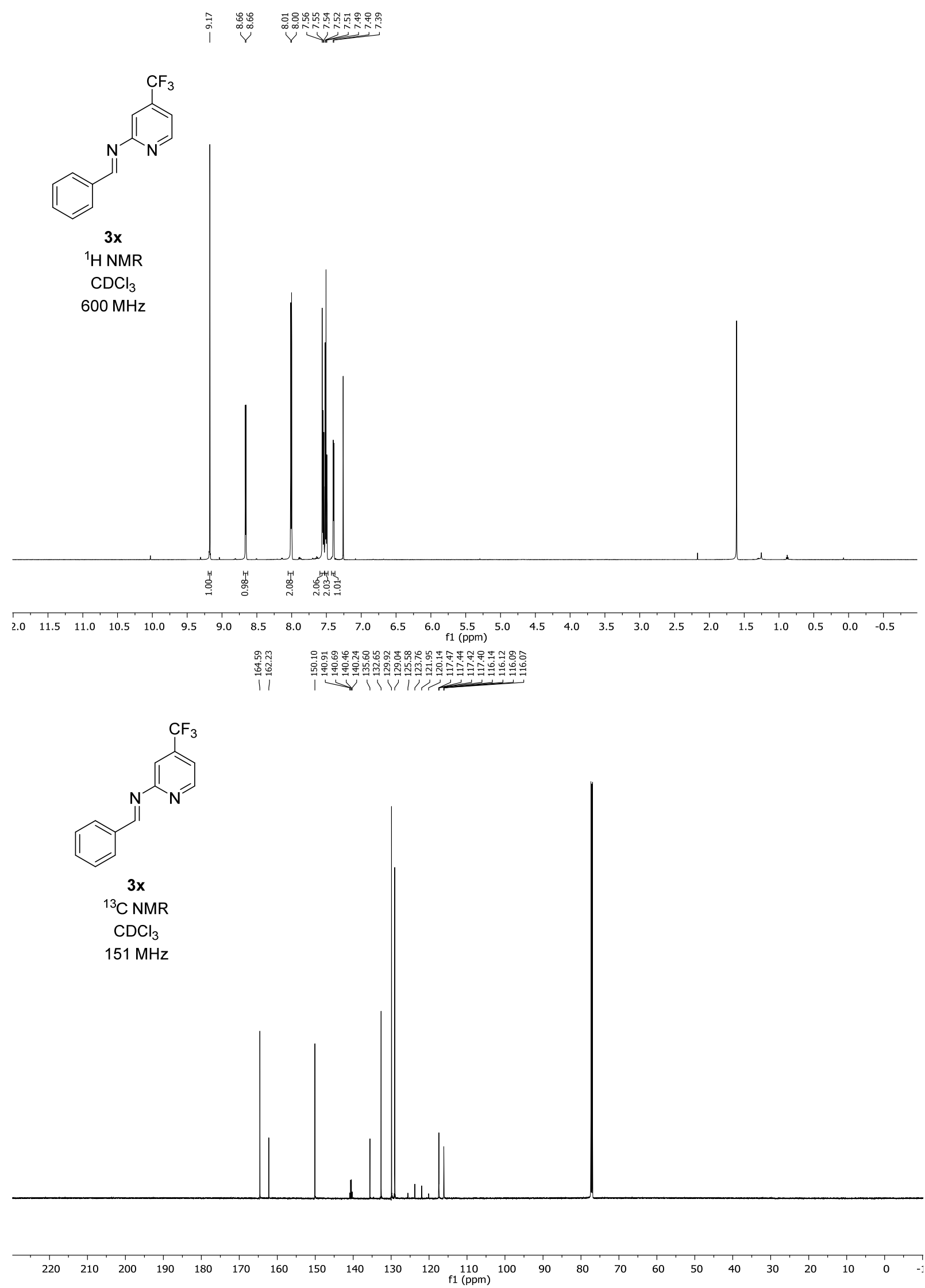


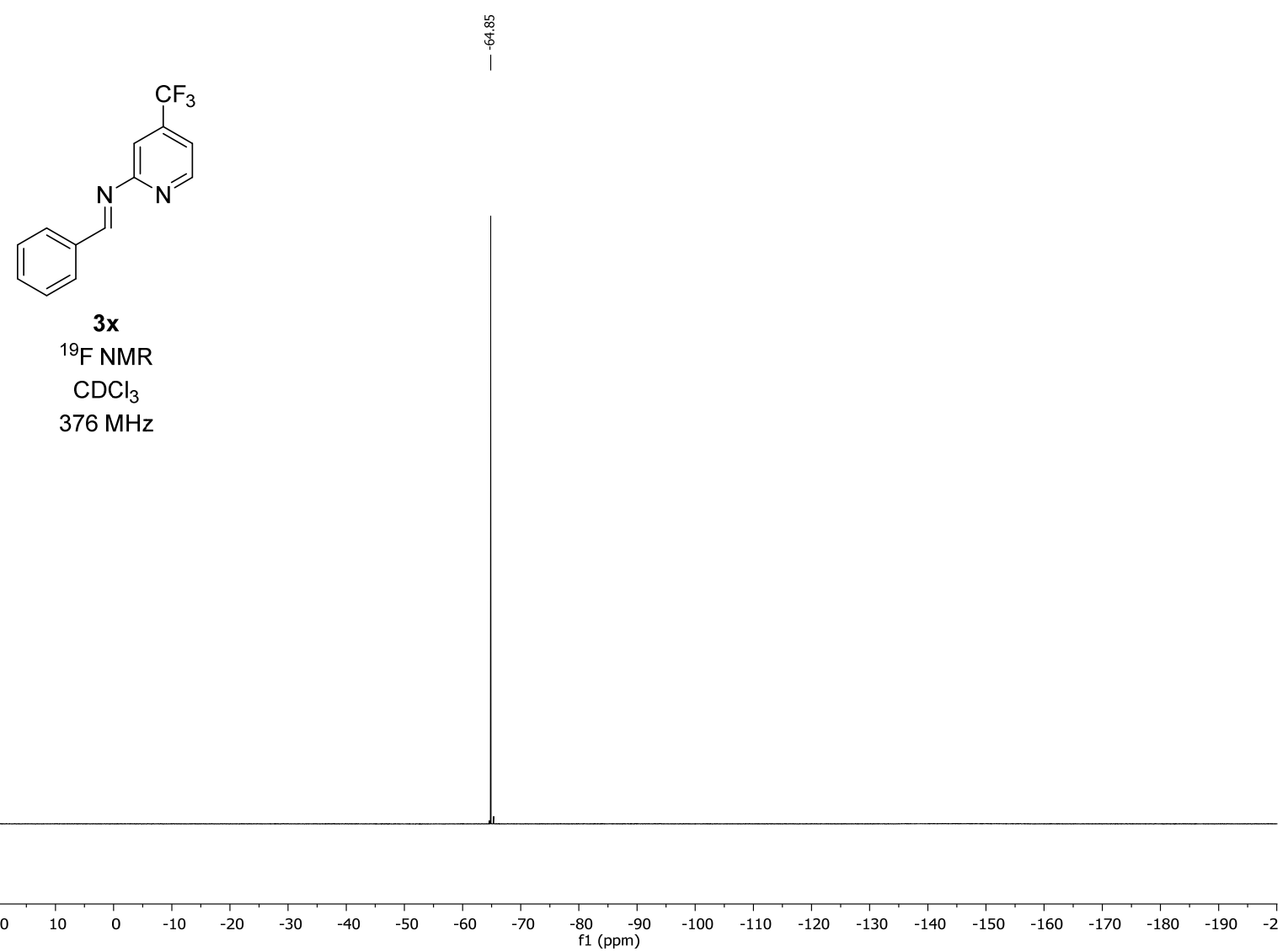

$\begin{array}{lllllllllllllllllllllllll}30 & 20 & 10 & 0 & -10 & -20 & -30 & -40 & -50 & -60 & -70 & -80 & -90 & -100 & -110 & -120 & -130 & -140 & -150 & -160 & -170 & -180 & -190 & -2\end{array}$ 


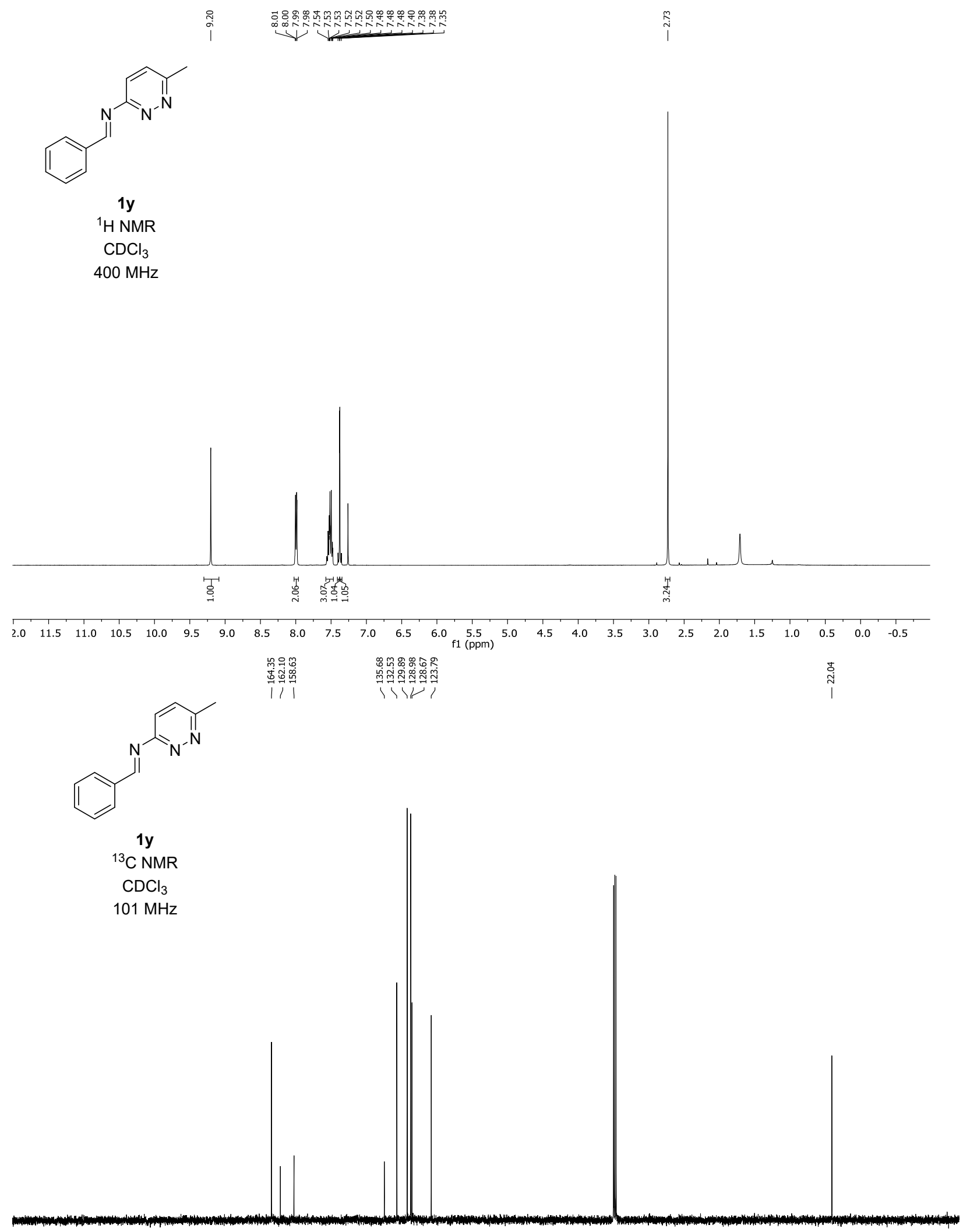

\begin{tabular}{llllllllllllllllllllllllllllllll}
\hline 30 & 220 & 210 & 200 & 190 & 180 & 170 & 160 & 150 & 140 & 130 & 120 & $\begin{array}{l}110 \\
\mathrm{f} 1\end{array}(\mathrm{ppm})$ & 100 & 90 & 80 & 70 & 60 & 50 & 40 & 30 & 20 & 10 & 0 & -1
\end{tabular} 


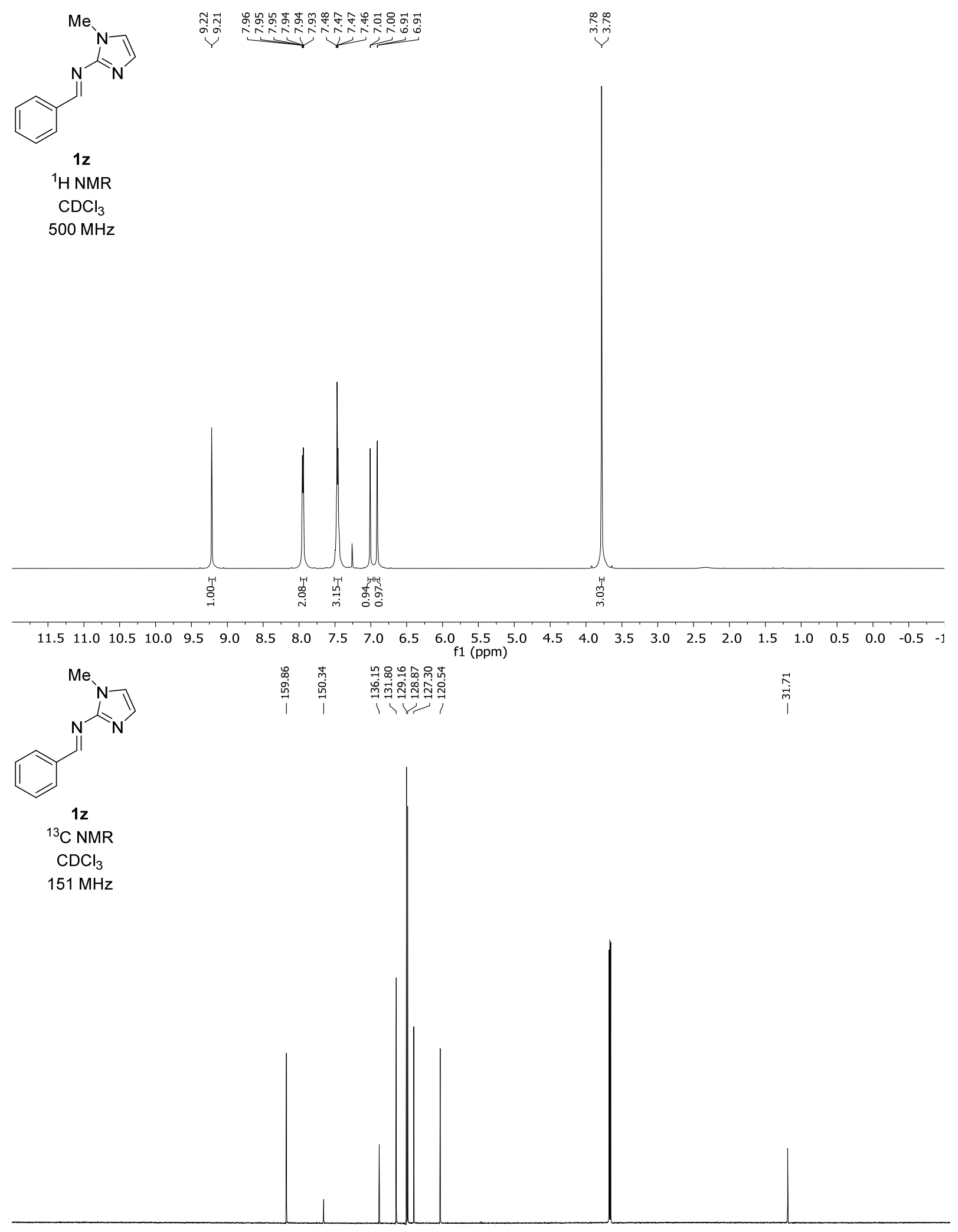

$\begin{array}{llllllllllllllllllllllllllllllllll}30 & 220 & 210 & 200 & 190 & 180 & 170 & 160 & 150 & 140 & 130 & 120 & 110 & 100 & 90 & 80 & 70 & 60 & 50 & 40 & 30 & 20 & 10 & 0 & -\end{array}$ 

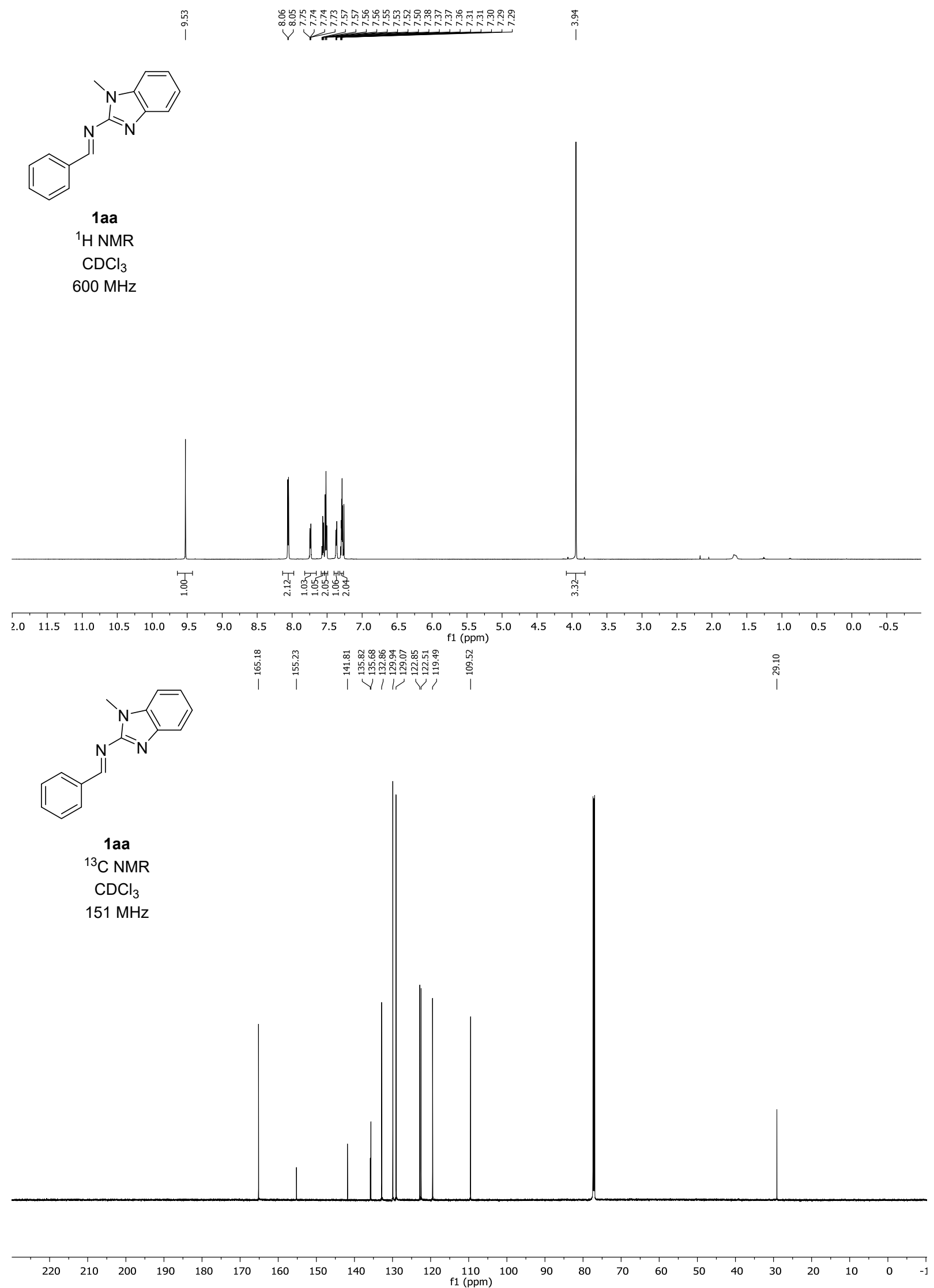

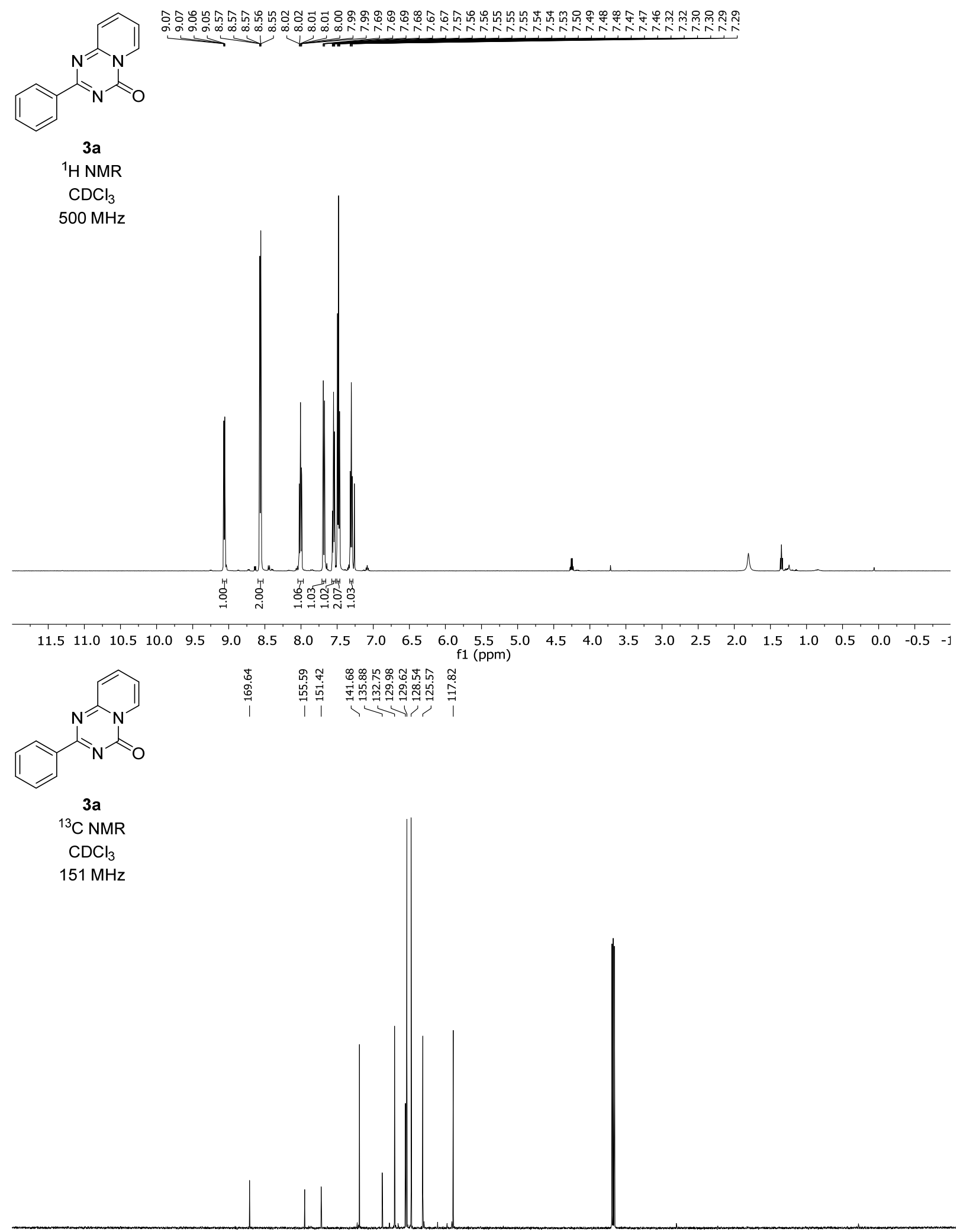

$\begin{array}{lllllllllllllllllllllllllll}30 & 220 & 210 & 200 & 190 & 180 & 170 & 160 & 150 & 140 & 130 & 120 & \begin{array}{l}110 \\ \mathrm{f} 1\end{array}(\mathrm{ppm}) & 100 & 90 & 80 & 70 & 60 & 50 & 40 & 30 & 20 & 10 & 0 & -\end{array}$ 


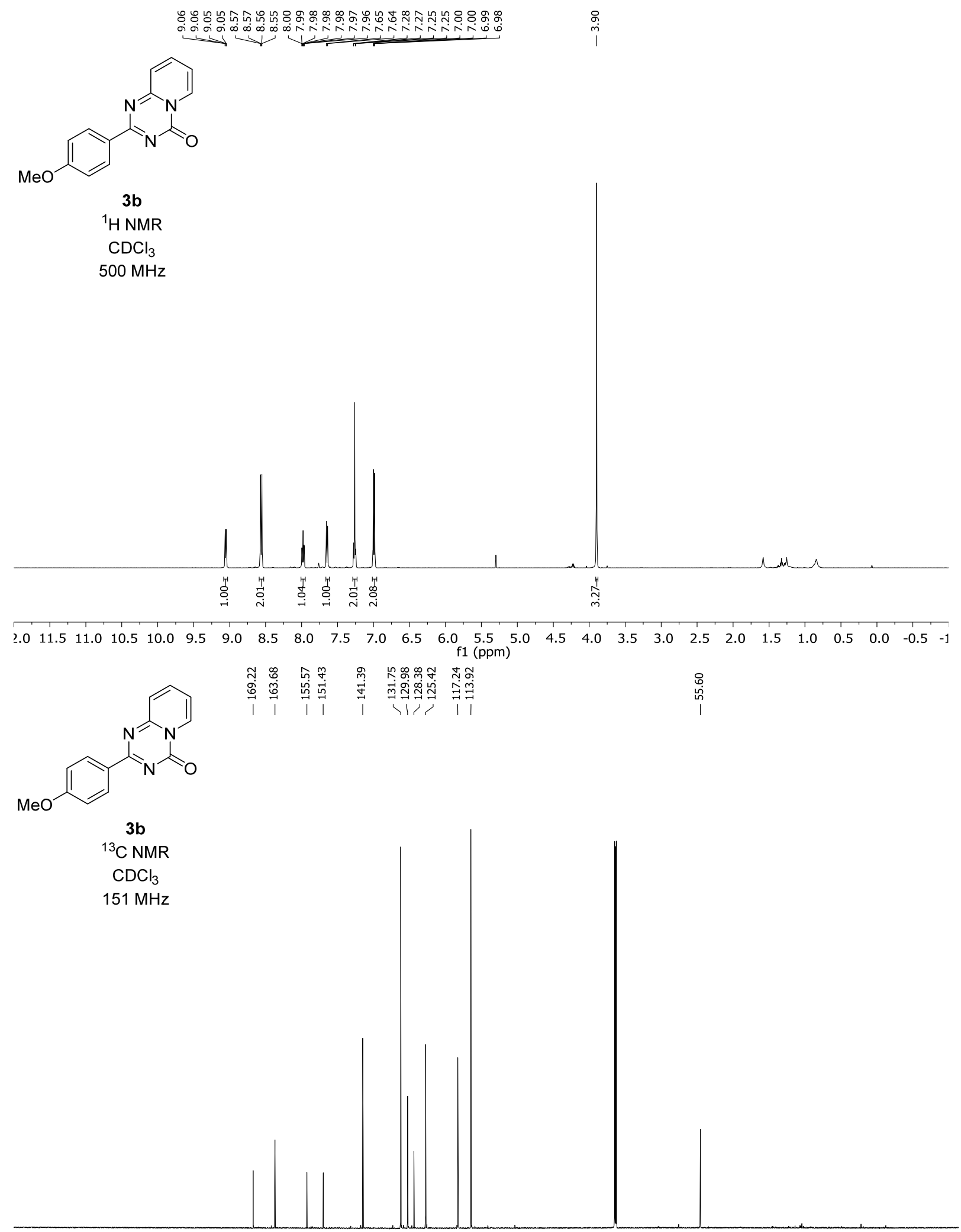

$\begin{array}{lllllllllllllllllllllllll}30 & 220 & 210 & 200 & 190 & 180 & 170 & 160 & 150 & 140 & 130 & 120 & 110 & 100 & 90 & 80 & 70 & 60 & 50 & 40 & 30 & 20 & 10 & 0 & -\end{array}$ 

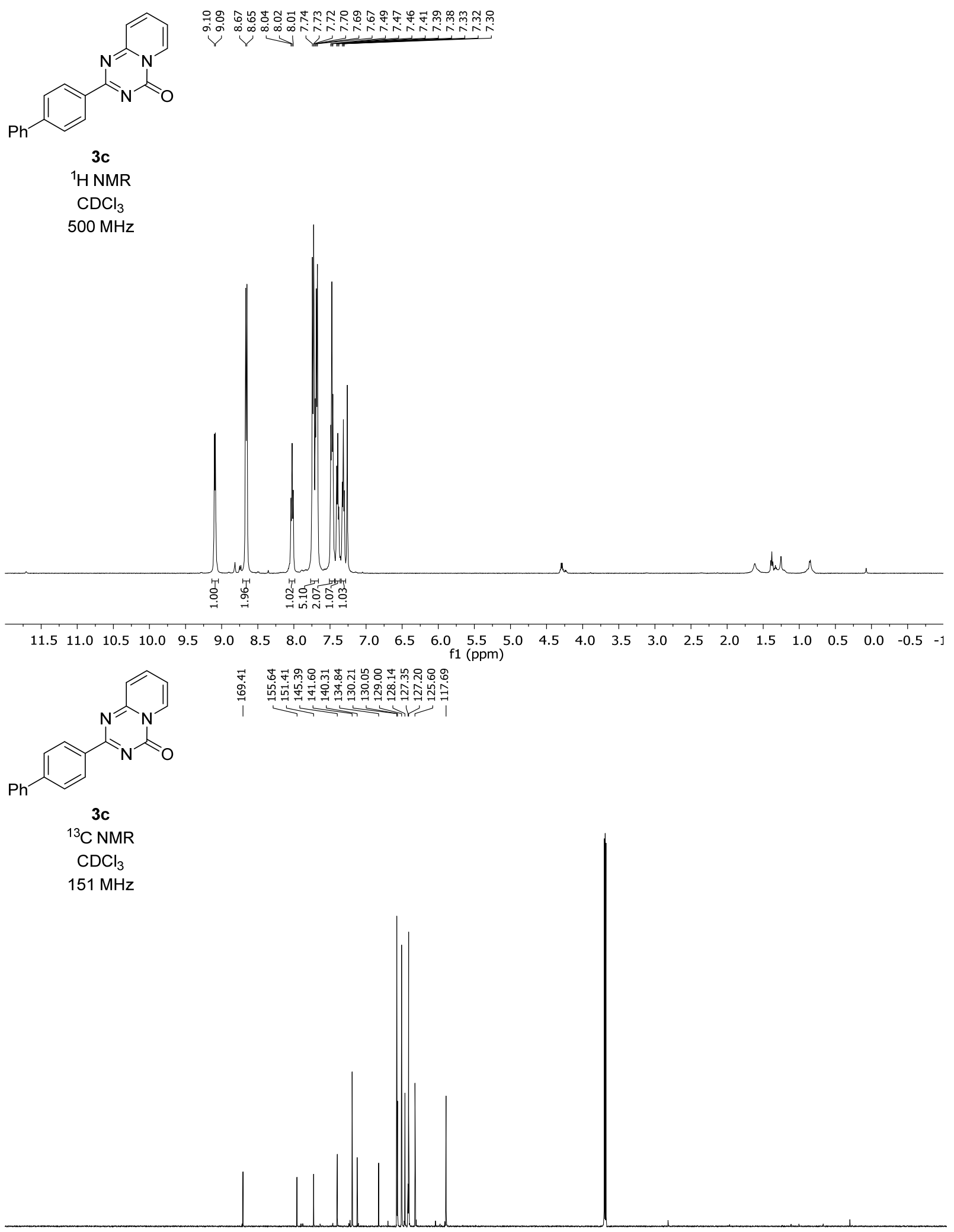

$\begin{array}{lllllllllllllllllllllllllllllll}30 & 220 & 210 & 200 & 190 & 180 & 170 & 160 & 150 & 140 & 130 & 120 & 110 & 100 & 90 & 80 & 70 & 60 & 50 & 40 & 30 & 20 & 10 & 0 & -\end{array}$ 

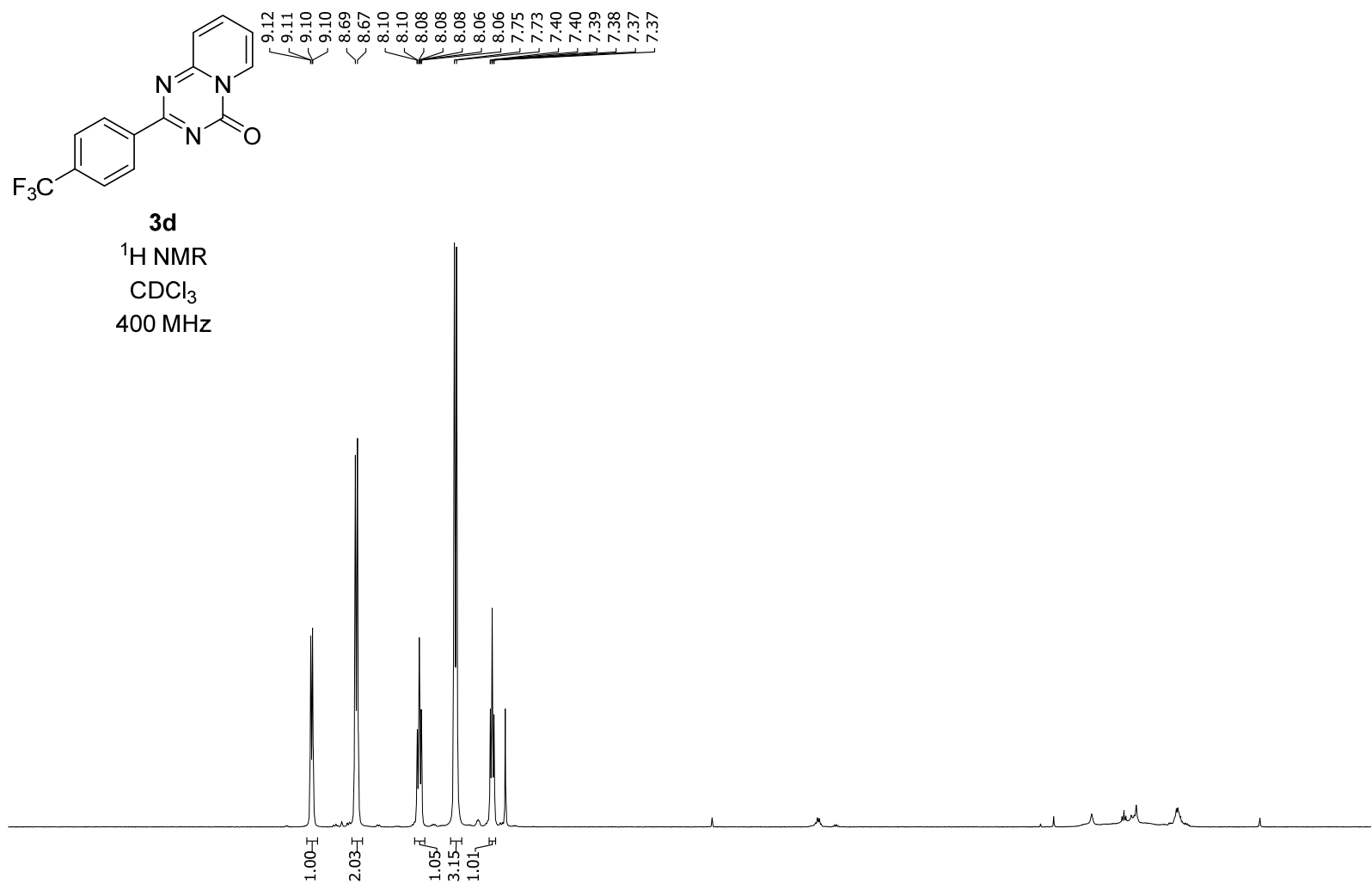

$\begin{array}{llllllllllllllllllllllllllllllllll}2.0 & 11.5 & 11.0 & 10.5 & 10.0 & 9.5 & 9.0 & 8.5 & 8.0 & 7.5 & 7.0 & 6.5 & 6.0 & \begin{array}{c}5.5 \\ \mathrm{f} 1\end{array}(\mathrm{ppm}) & 5.0 & 4.5 & 4.0 & 3.5 & 3.0 & 2.5 & 2.0 & 1.5 & 1.0 & 0.5 & 0.0 & -0.5 & -1\end{array}$<smiles>O=c1nc(-c2ccc(C(F)(F)F)cc2)nc2ccccn12</smiles>

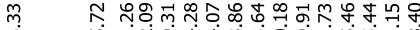

至

3d

${ }^{13} \mathrm{C}$ NMR

$\mathrm{CDCl}_{3}$

$151 \mathrm{MHz}$

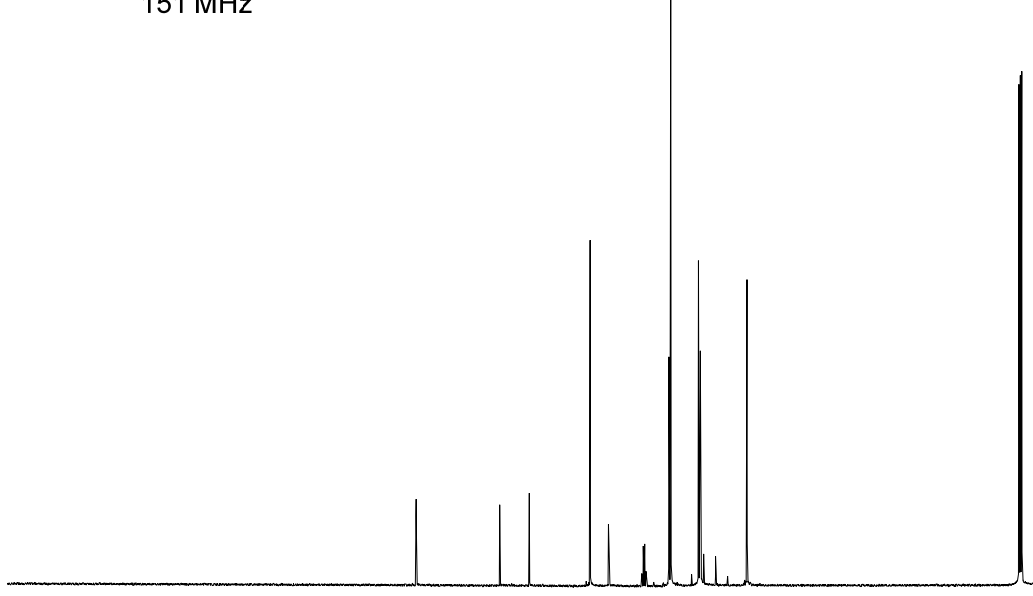

$\begin{array}{llllllllllllllllllllllllll}30 & 220 & 210 & 200 & 190 & 180 & 170 & 160 & 150 & 140 & 130 & 120 & \begin{array}{l}110 \\ \mathrm{f} 1\end{array}(\mathrm{ppm}) & 100 & 90 & 80 & 70 & 60 & 50 & 40 & 30 & 20 & 10 & 0 & -\end{array}$ 


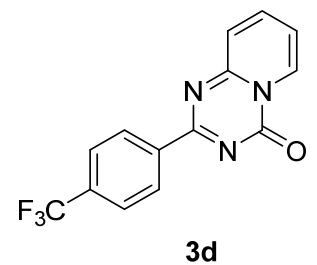

${ }^{19} \mathrm{~F} \mathrm{NMR}$

$\mathrm{CDCl}_{3}$

$376 \mathrm{MHz}$ 


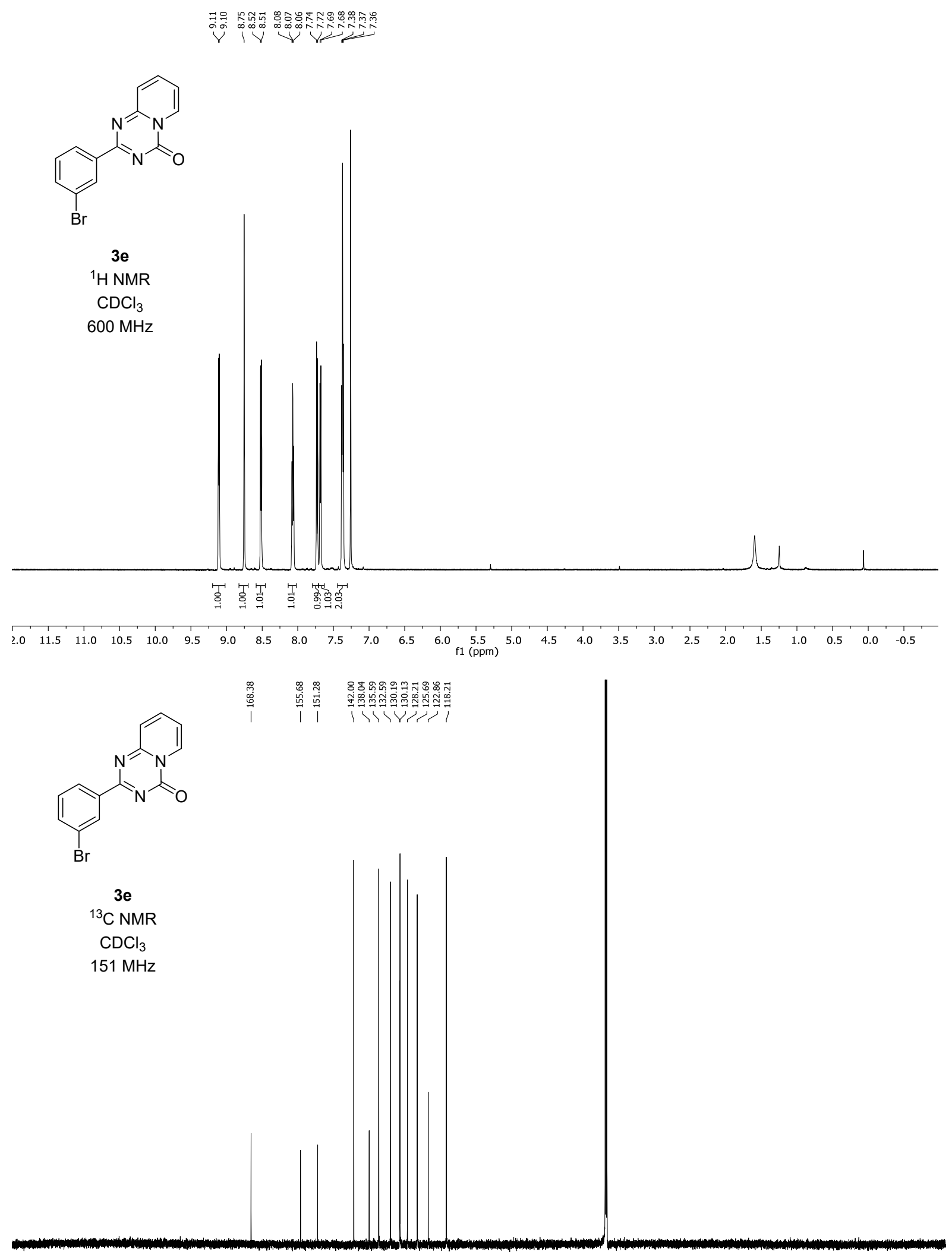

$\begin{array}{llllllllllllllllllllllllllllll}220 & 210 & 200 & 190 & 180 & 170 & 160 & 150 & 140 & 130 & 120 & \begin{array}{c}110 \\ \mathrm{f} 1(\mathrm{ppm})\end{array} & 100 & 90 & 80 & 70 & 60 & 50 & 40 & 30 & 20 & 10 & 0 & -:\end{array}$ 


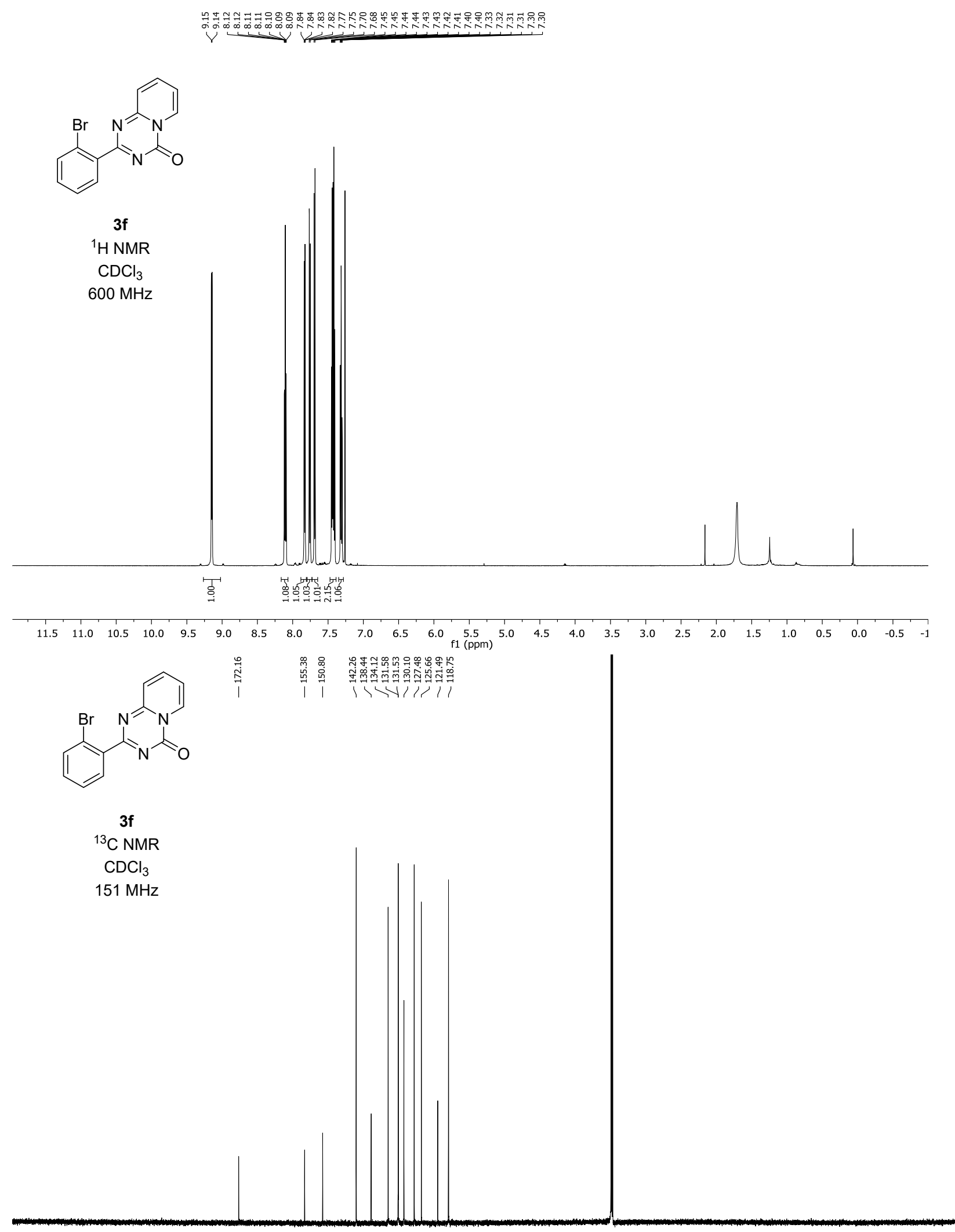

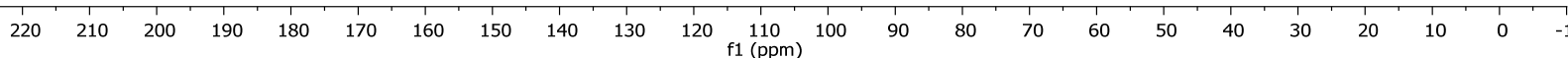



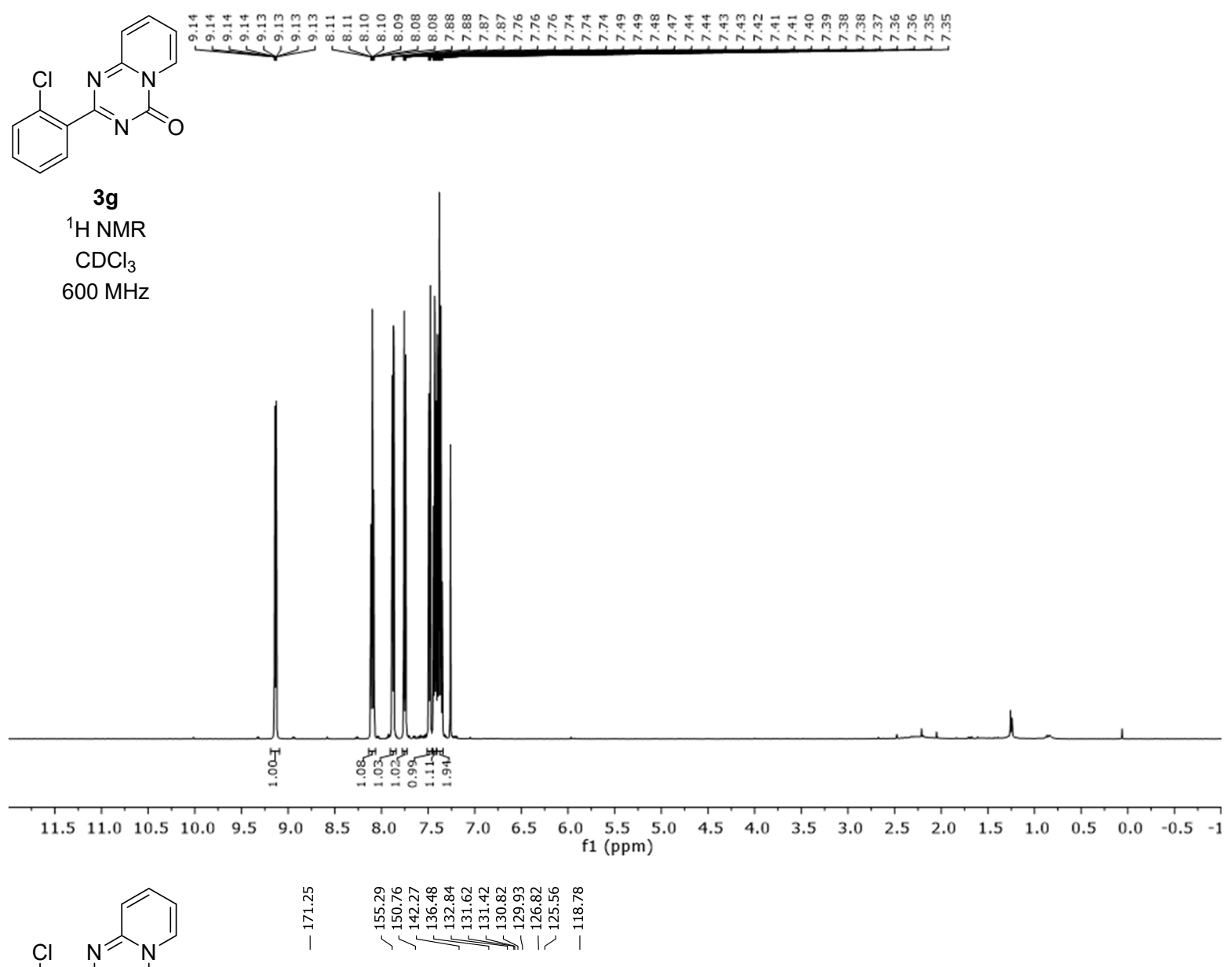

$\mathrm{N}_{\mathrm{N}}$

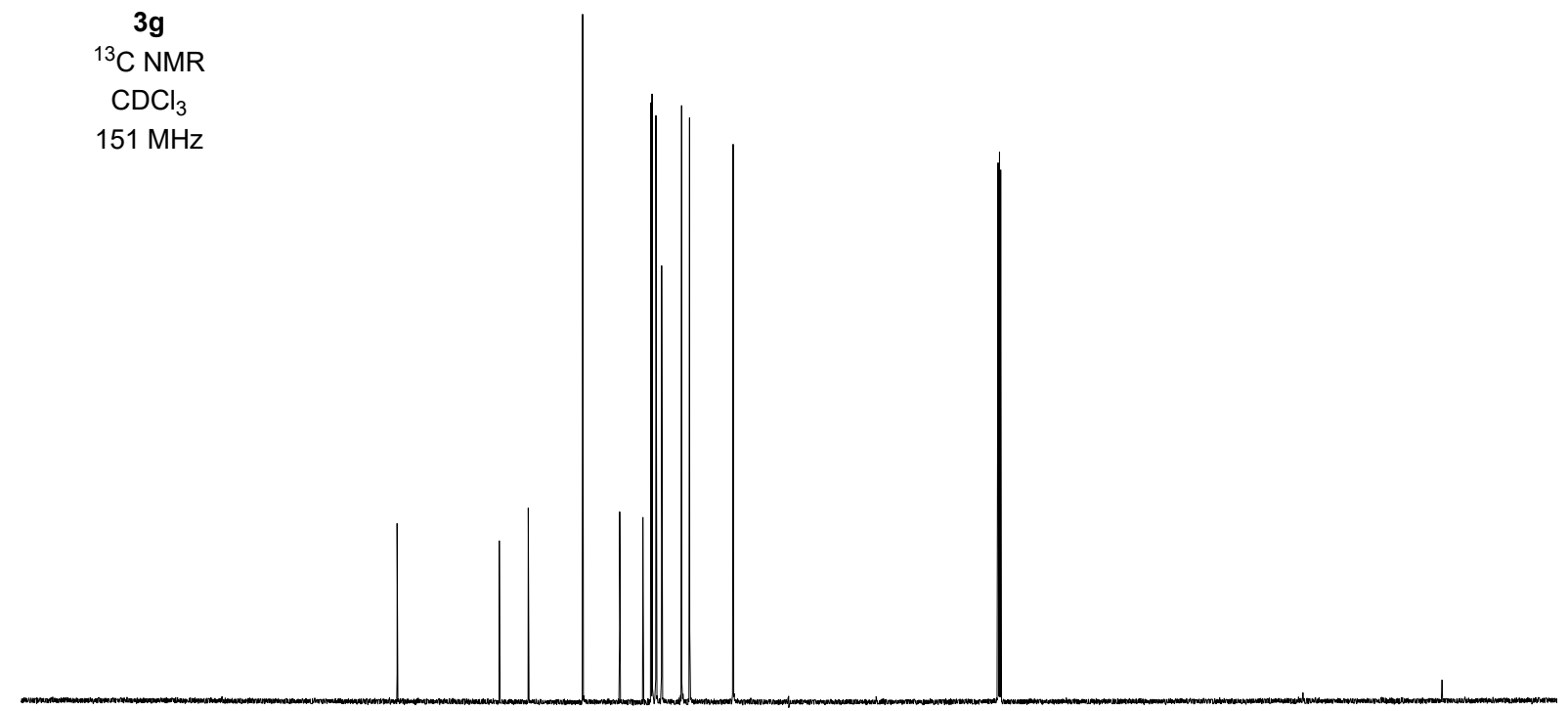

$\begin{array}{lllllllllllllllllllllll}30 & 220 & 210 & 200 & 190 & 180 & 170 & 160 & 150 & 140 & 130 & 120 \begin{array}{c}110 \\ \mathrm{f} 1(\mathrm{ppm})\end{array} & 100 & 90 & 80 & 70 & 60 & 50 & 40 & 30 & 20 & 10 & 0\end{array}$ 

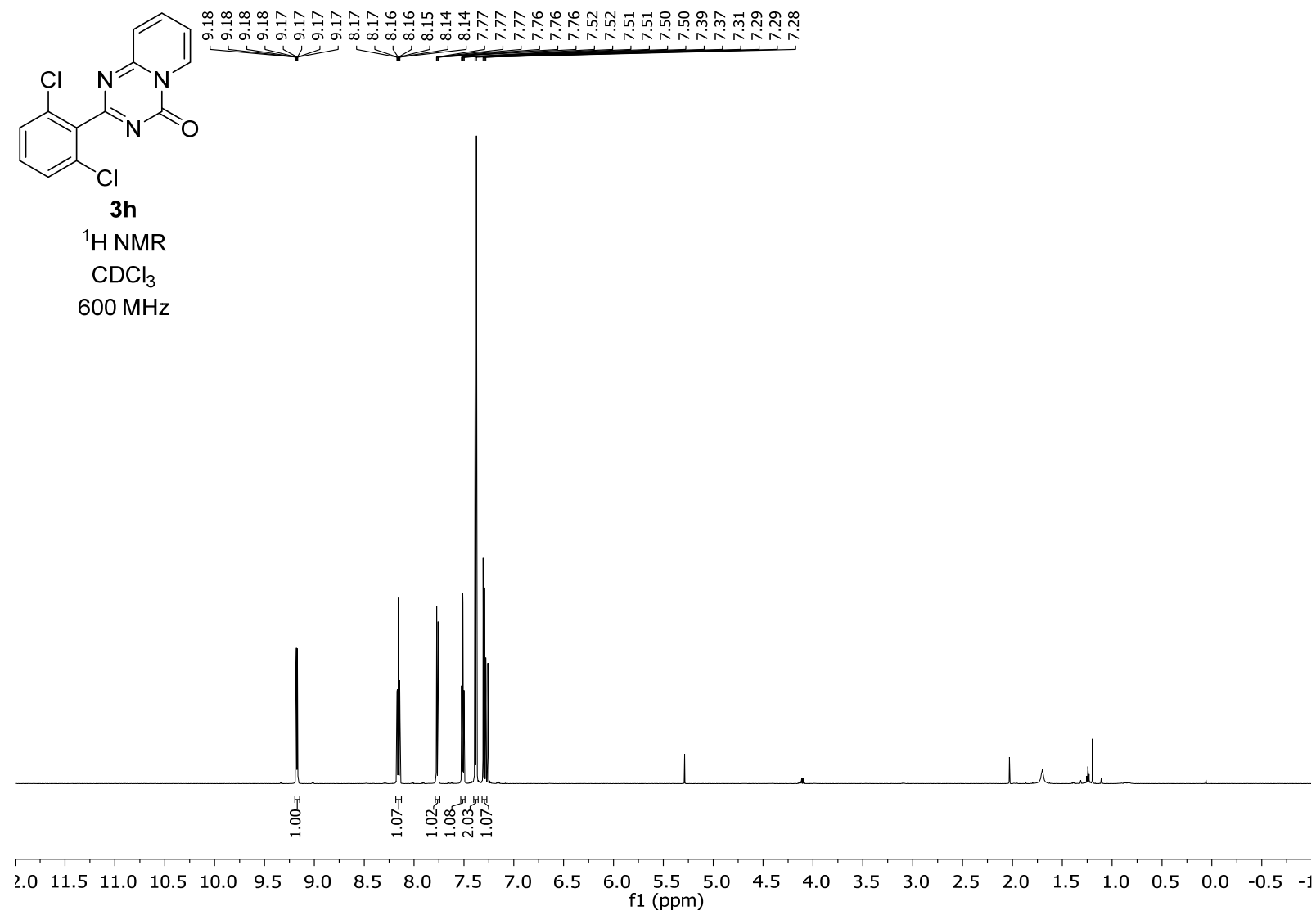<smiles>O=c1nc(-c2c(Cl)cccc2Cl)nc2ccccn12</smiles>

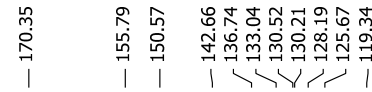

$3 \mathrm{~h}$

${ }^{13} \mathrm{C}$ NMR

$\mathrm{CDCl}_{3}$

$151 \mathrm{MHz}$

$\begin{array}{lllllllllllllllllllllllllllllllllllllll}30 & 220 & 210 & 200 & 190 & 180 & 170 & 160 & 150 & 140 & 130 & 120 & 110 & 100 & 90 & 80 & 70 & 60 & 50 & 40 & 30 & 20 & 10 & 0 & -\end{array}$ 


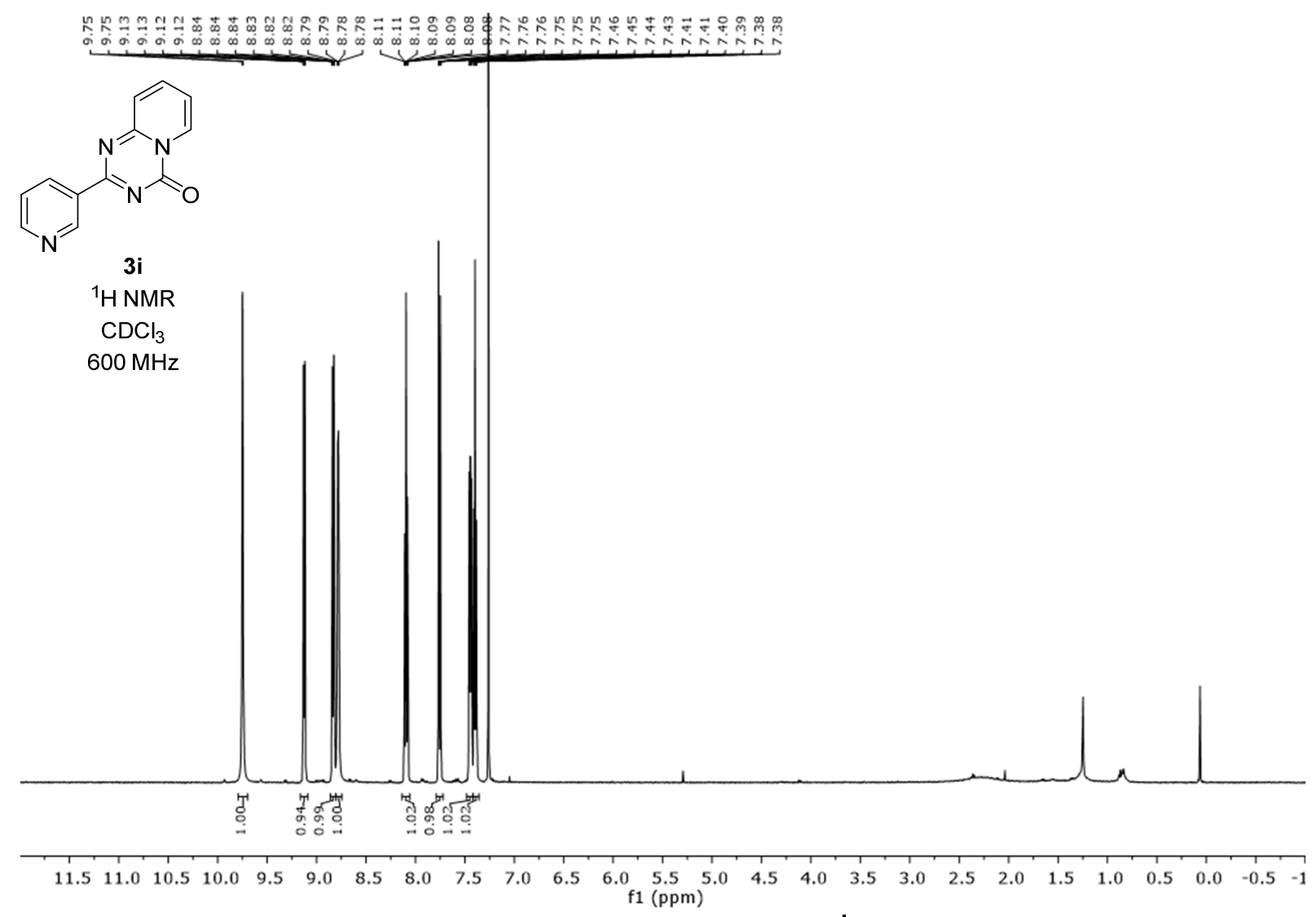

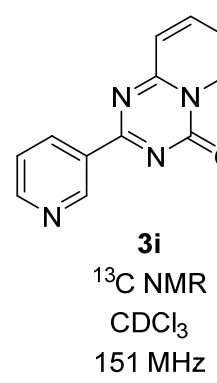

|

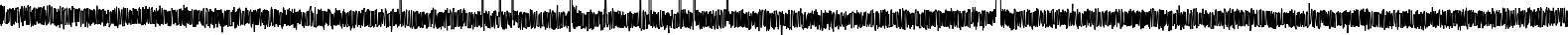

$\begin{array}{llllllllllllllllllllllllllll}30 & 220 & 210 & 200 & 190 & 180 & 170 & 160 & 150 & 140 & 130 & 120 & 110 & 100 & 90 & 80 & 70 & 60 & 50 & 40 & 30 & 20 & 10 & 0 & -\end{array}$ 


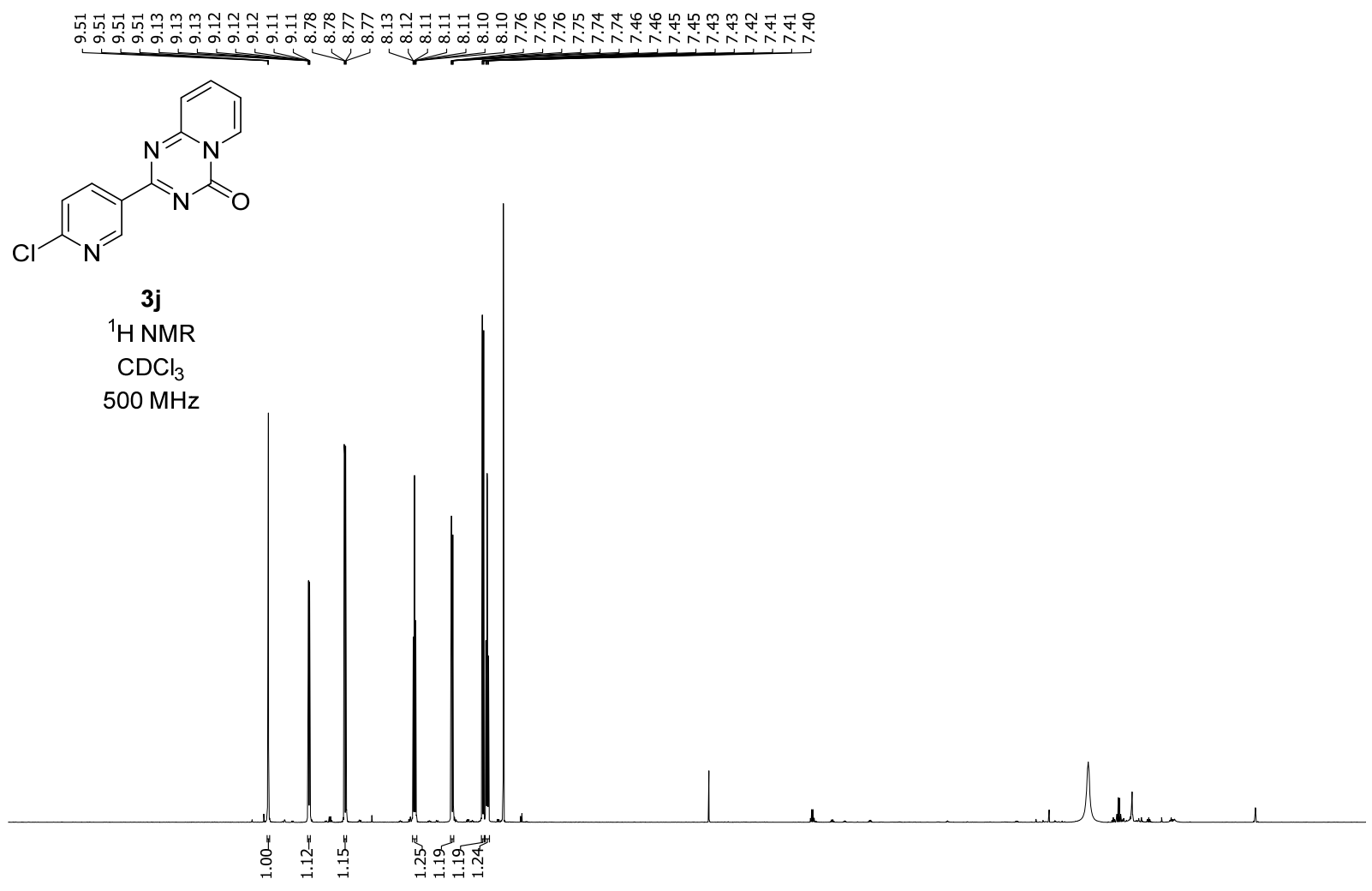

$\begin{array}{lllllllllllllllllllllllllllllllll}2.0 & 11.5 & 11.0 & 10.5 & 10.0 & 9.5 & 9.0 & 8.5 & 8.0 & 7.5 & 7.0 & 6.5 & 6.0 & \begin{array}{c}5.5 \\ \mathrm{f} 1\end{array}(\mathrm{ppm}) & 5.0 & 4.5 & 4.0 & 3.5 & 3.0 & 2.5 & 2.0 & 1.5 & 1.0 & 0.5 & 0.0 & -0.5 & -1\end{array}$<smiles>O=c1nc(-c2ccc(Cl)nc2)nc2ccccn12</smiles>

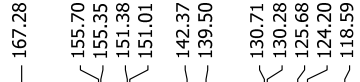

3j

${ }^{13} \mathrm{C}$ NMR

$\mathrm{CDCl}_{3}$

$151 \mathrm{MHz}$

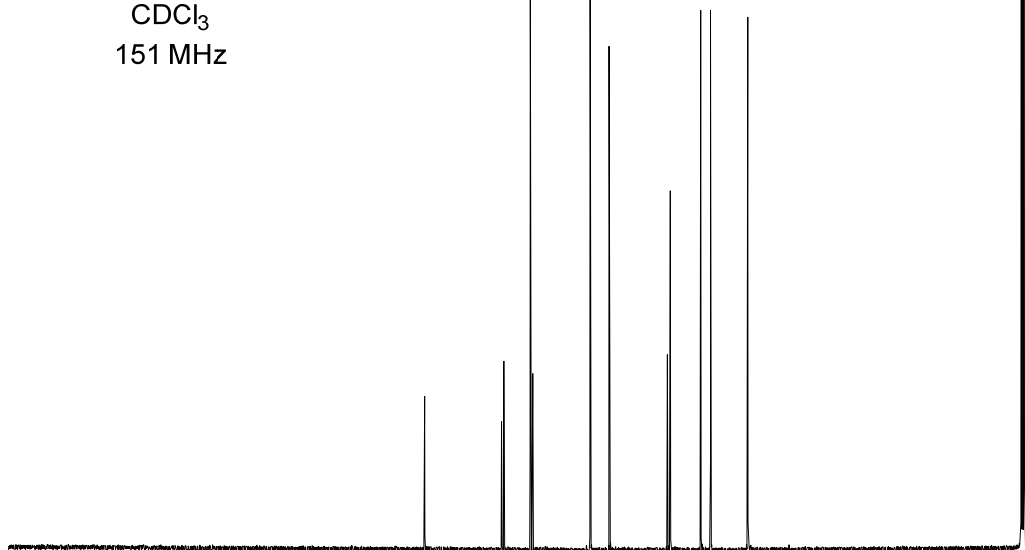

\begin{tabular}{lllllllllllllllllllllllllllll}
\hline 30 & 220 & 210 & 200 & 190 & 180 & 170 & 160 & 150 & 140 & 130 & 120 & $\begin{array}{l}110 \\
\mathrm{f} 1\end{array}(\mathrm{ppm})$ & 100 & 90 & 80 & 70 & 60 & 50 & 40 & 30 & 20 & 10 & 0 & -
\end{tabular} 


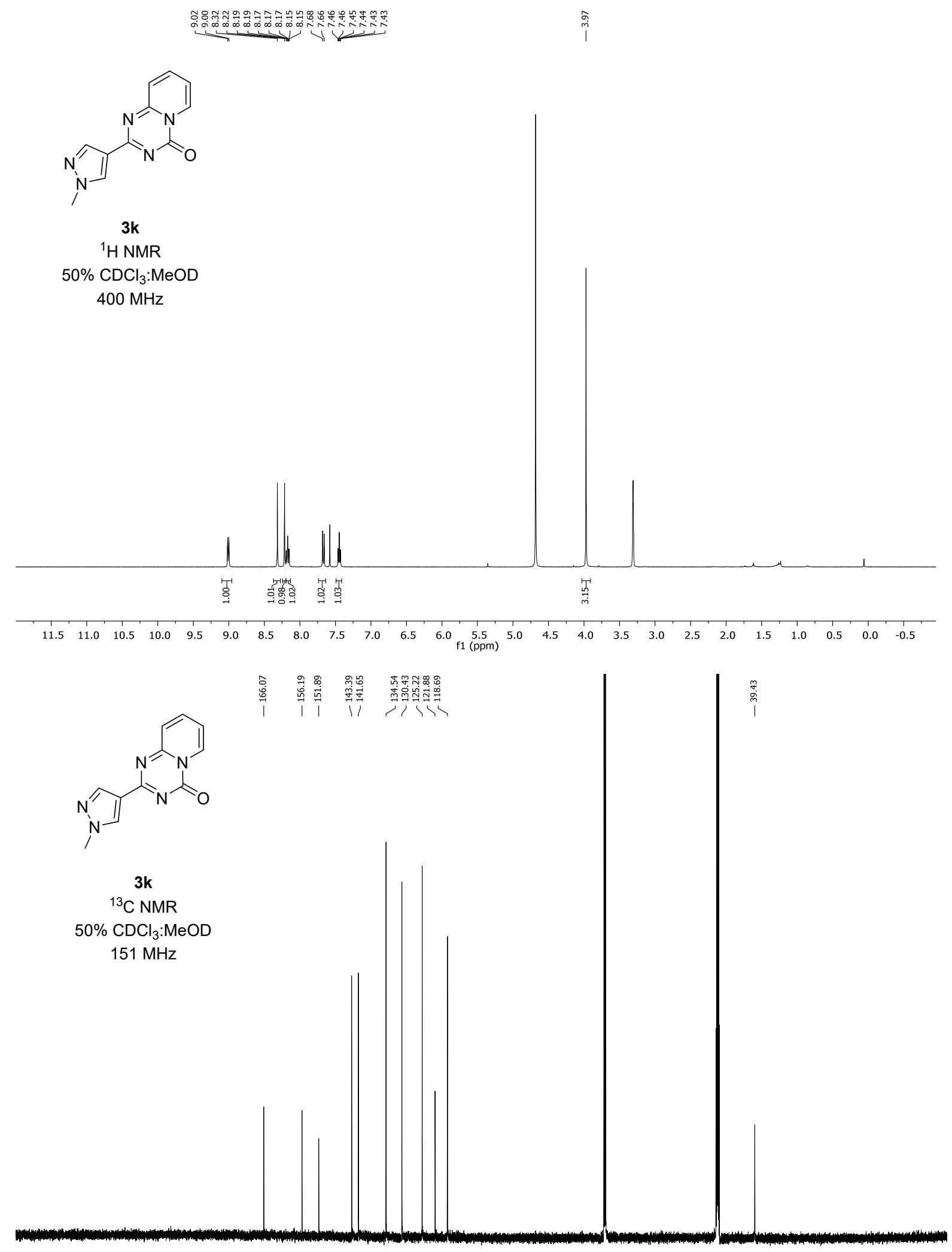

$\begin{array}{llllllllllllllllllllllllllll}220 & 210 & 200 & 190 & 180 & 170 & 160 & 150 & 140 & 130 & 120 & 110 & 100 & 90 & 80 & 70 & 60 & 50 & 40 & 30 & 20 & 10 & 0 & -1\end{array}$ 


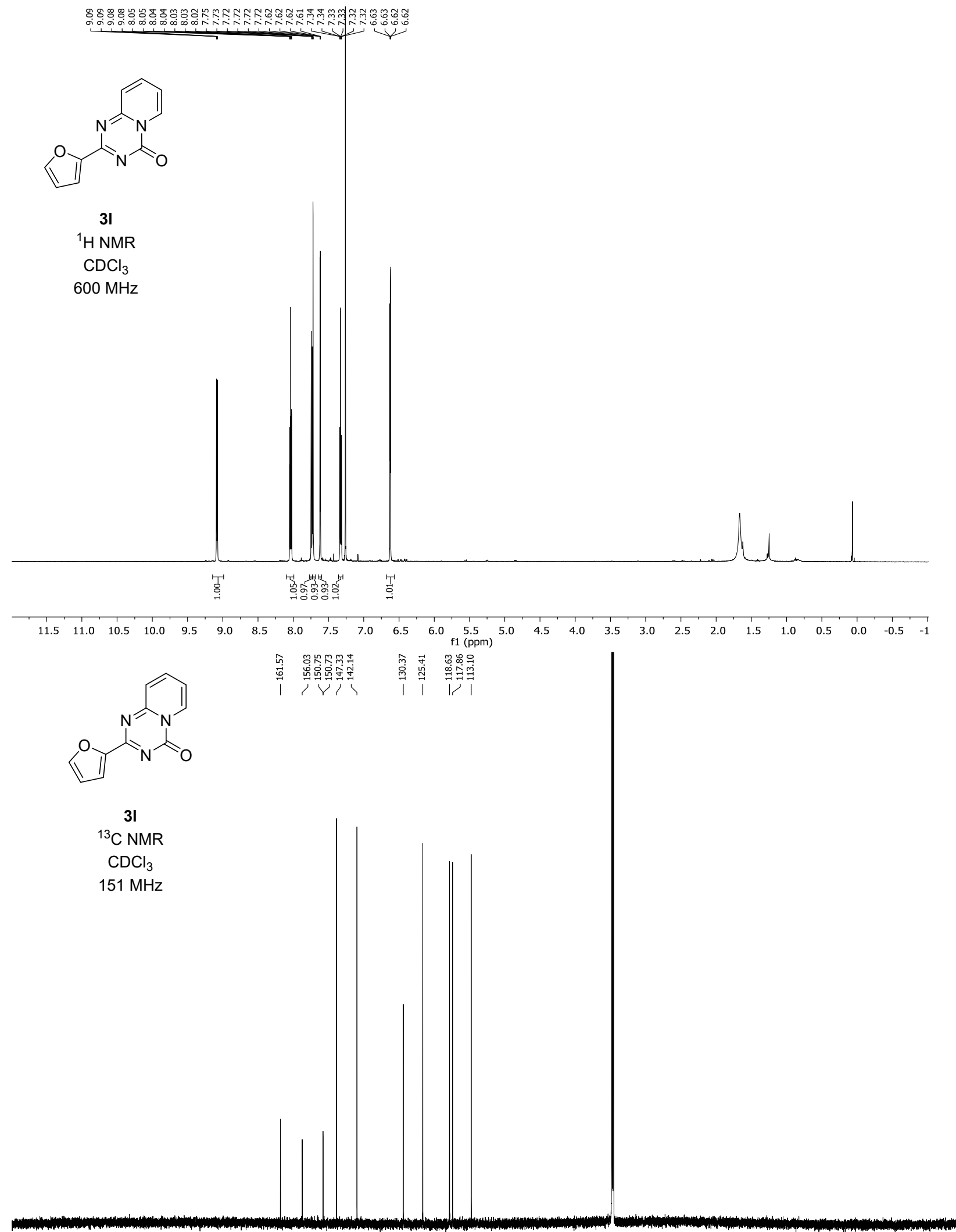

$\begin{array}{lllllllllllllllllllllllllllllll}220 & 210 & 200 & 190 & 180 & 170 & 160 & 150 & 140 & 130 & 120 & 110 & 100 & 90 & 80 & 70 & 60 & 50 & 40 & 30 & 20 & 10 & 0 & -\end{array}$ 


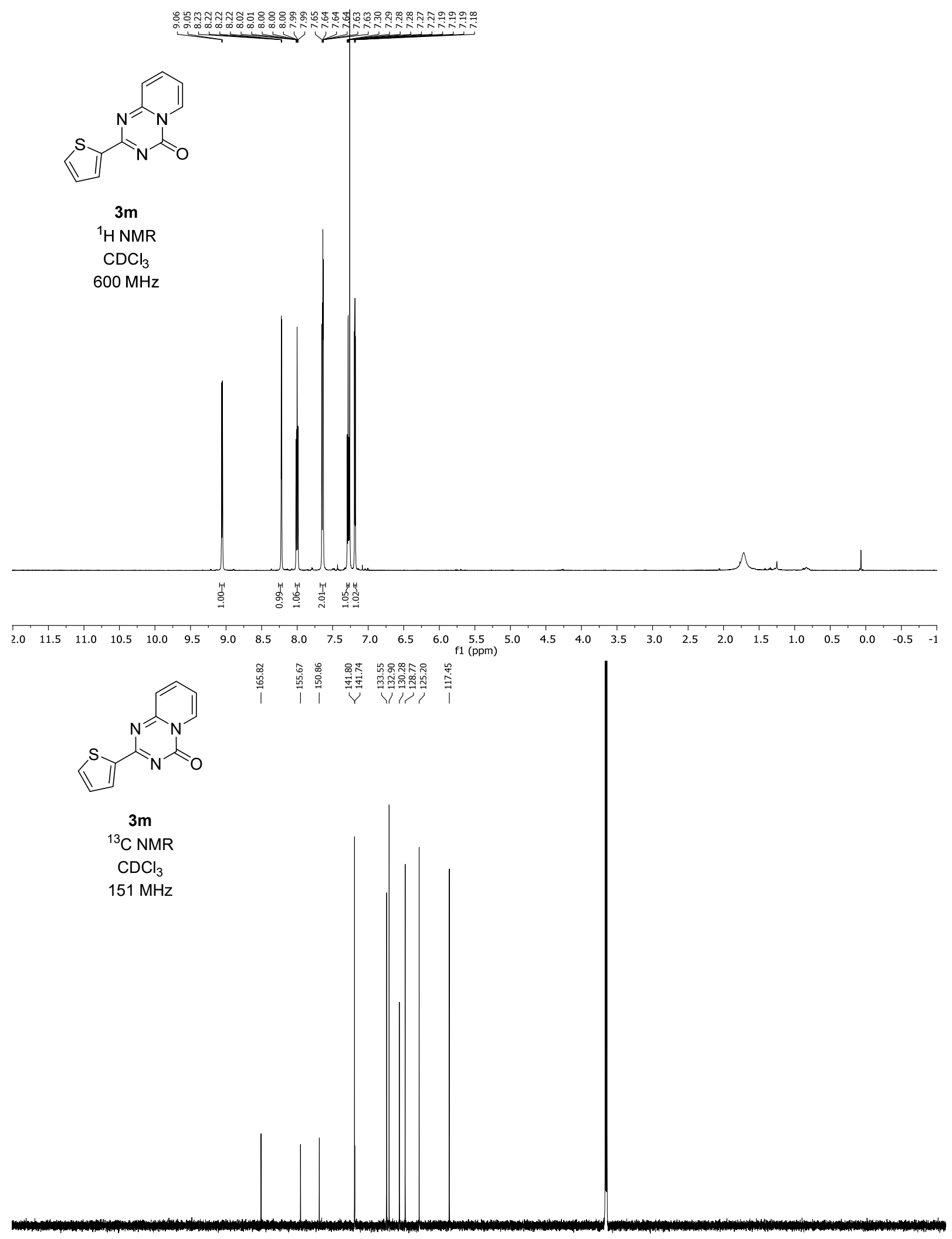

$\begin{array}{lllllllllllllllllllllllllllll}220 & 210 & 200 & 190 & 180 & 170 & 160 & 150 & 140 & 130 & 120 & \begin{array}{l}110 \\ \mathrm{f} 1(\mathrm{ppm})\end{array} & 100 & 90 & 80 & 70 & 60 & 50 & 40 & 30 & 20 & 10 & 0 & -:\end{array}$ 

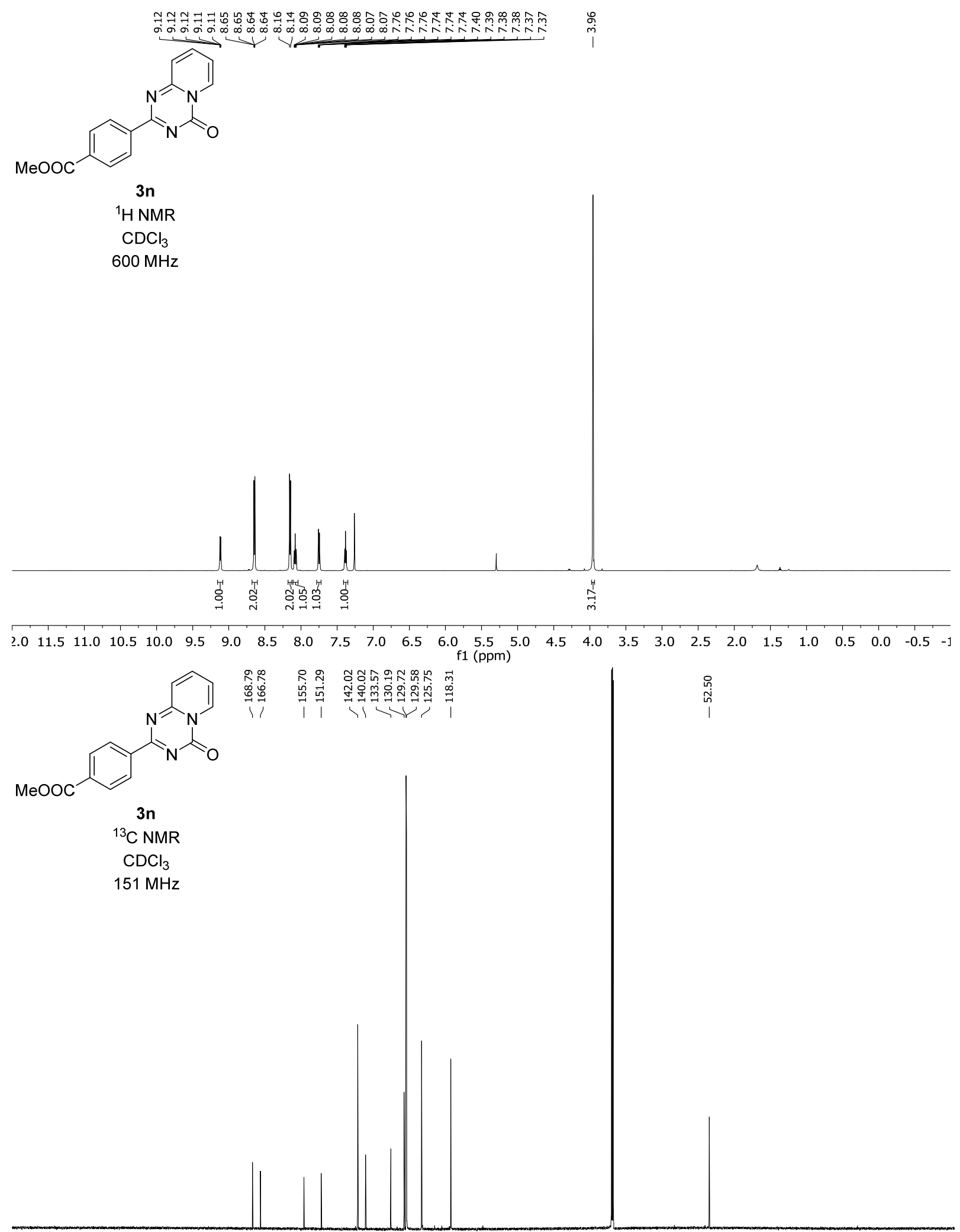

$\begin{array}{llllllllllllllllllllllllllllll}30 & 220 & 210 & 200 & 190 & 180 & 170 & 160 & 150 & 140 & 130 & 120 & 110 & 100 & 90 & 80 & 70 & 60 & 50 & 40 & 30 & 20 & 10 & 0 & -\end{array}$ 


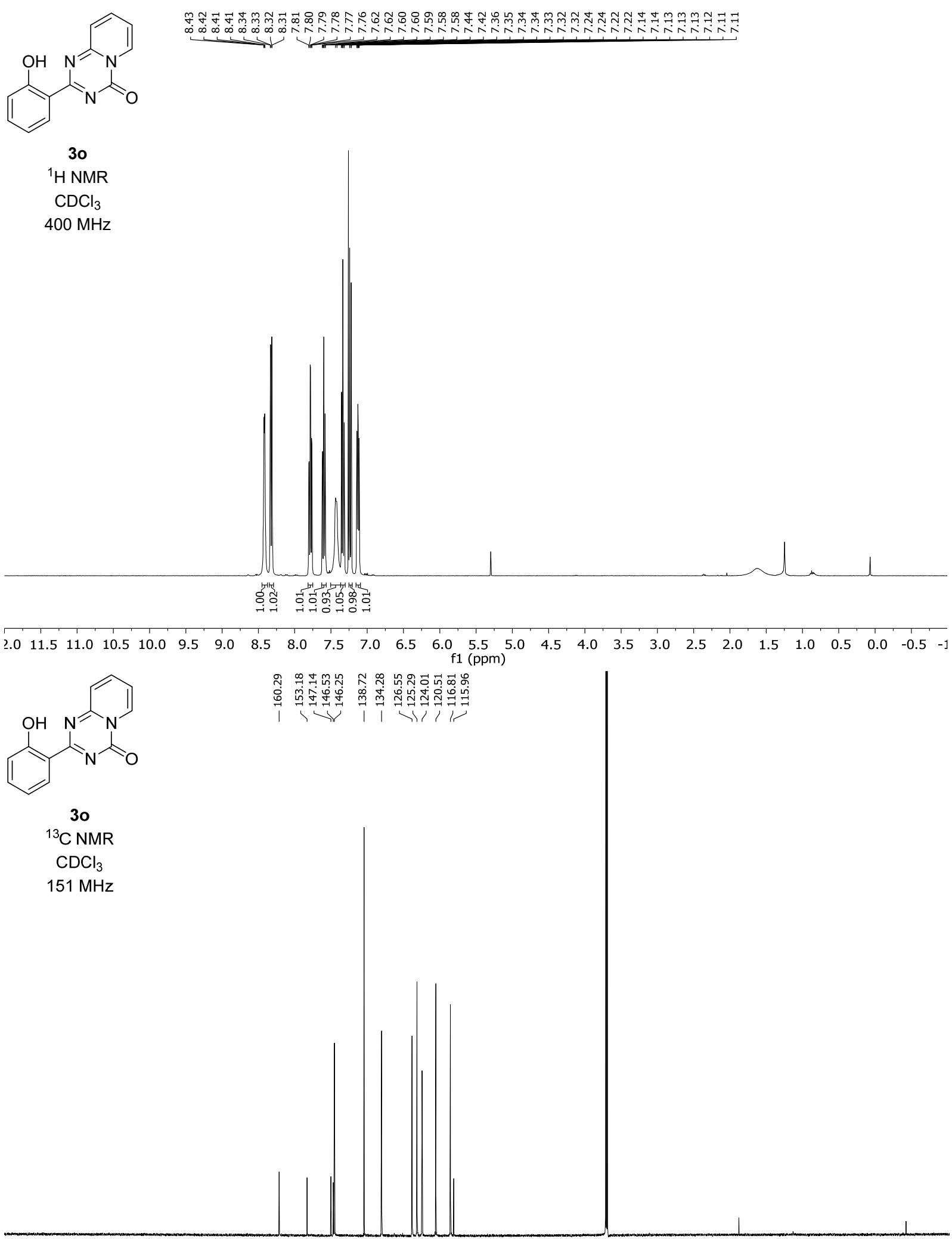

\begin{tabular}{lllllllllllllllllllllllllll}
\hline 30 & 220 & 210 & 200 & 190 & 180 & 170 & 160 & 150 & 140 & 130 & 120 & 110 & 100 & 90 & 80 & 70 & 60 & 50 & 40 & 30 & 20 & 10 & 0 & -
\end{tabular} 


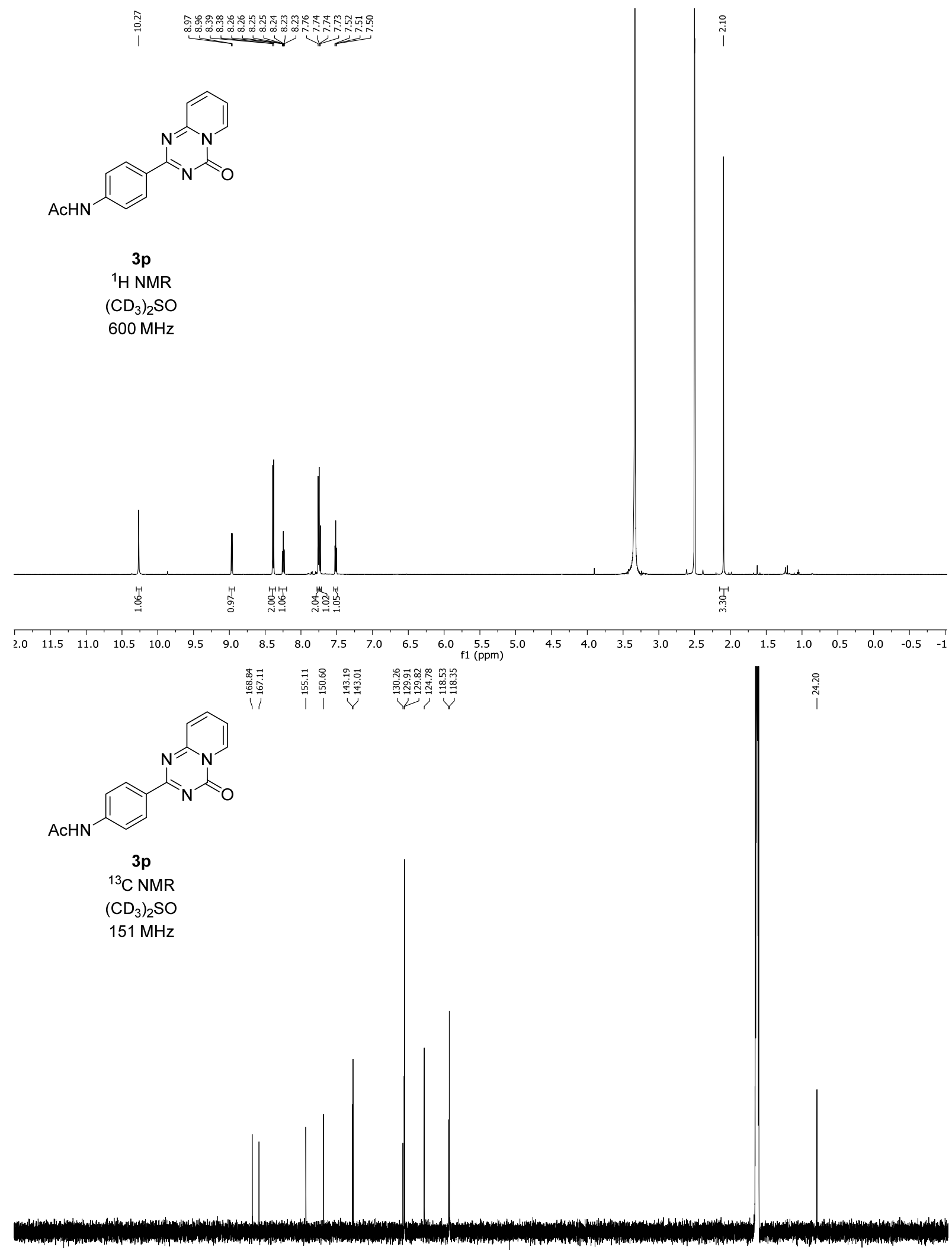

 


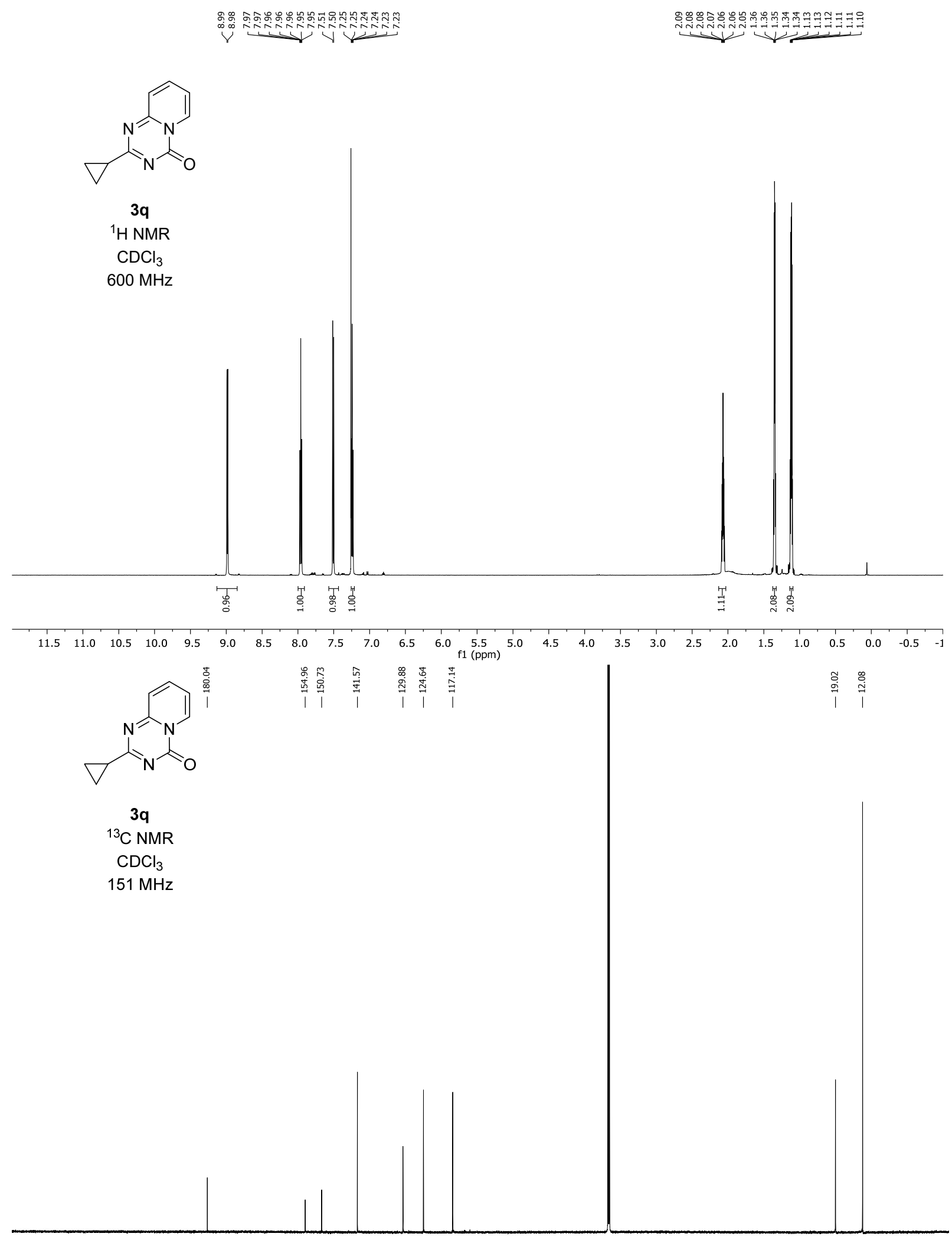

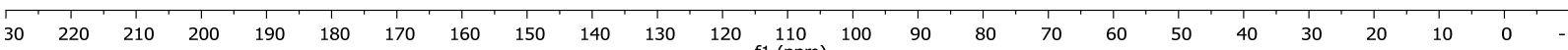




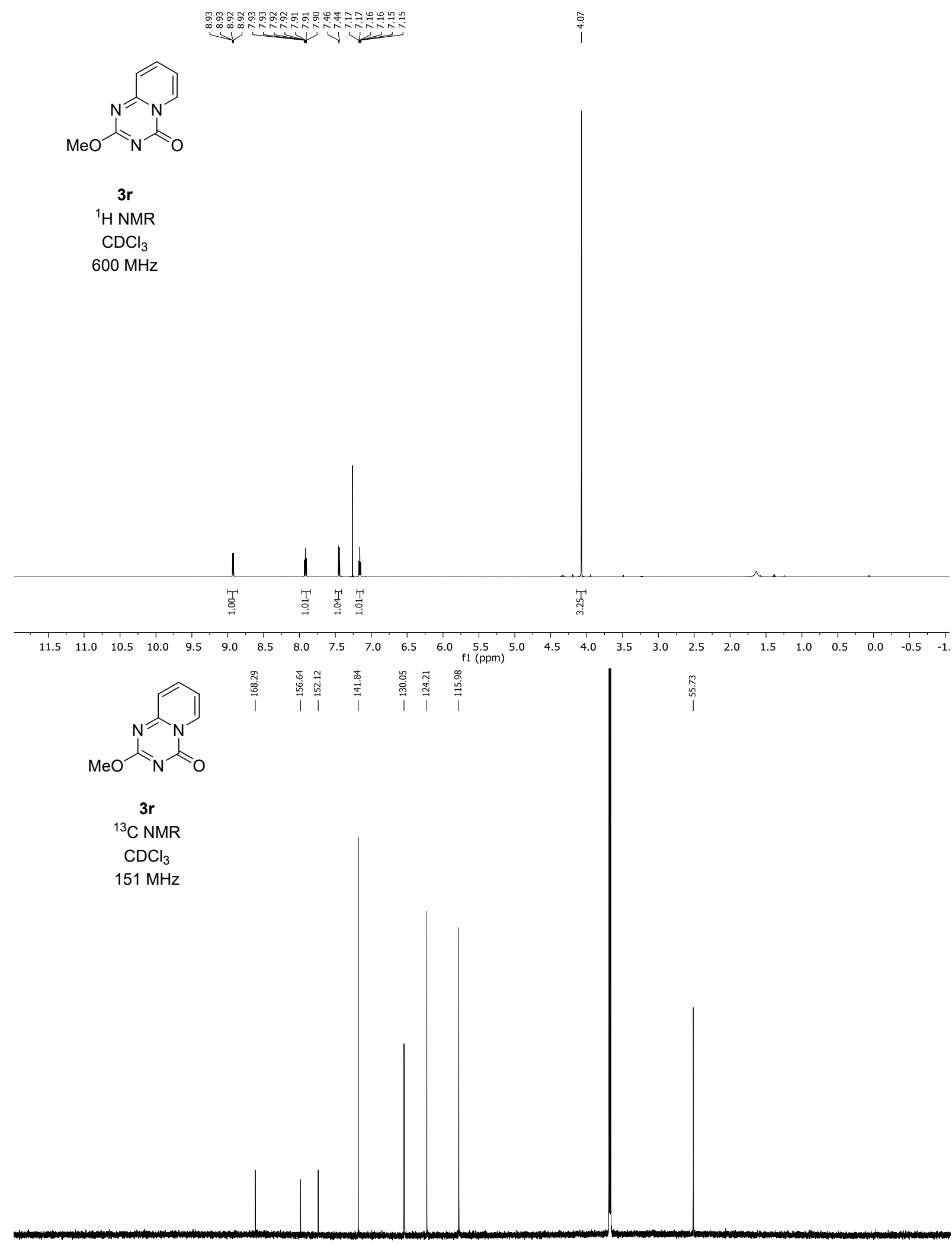

MeO

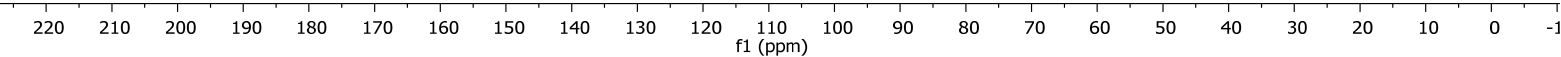



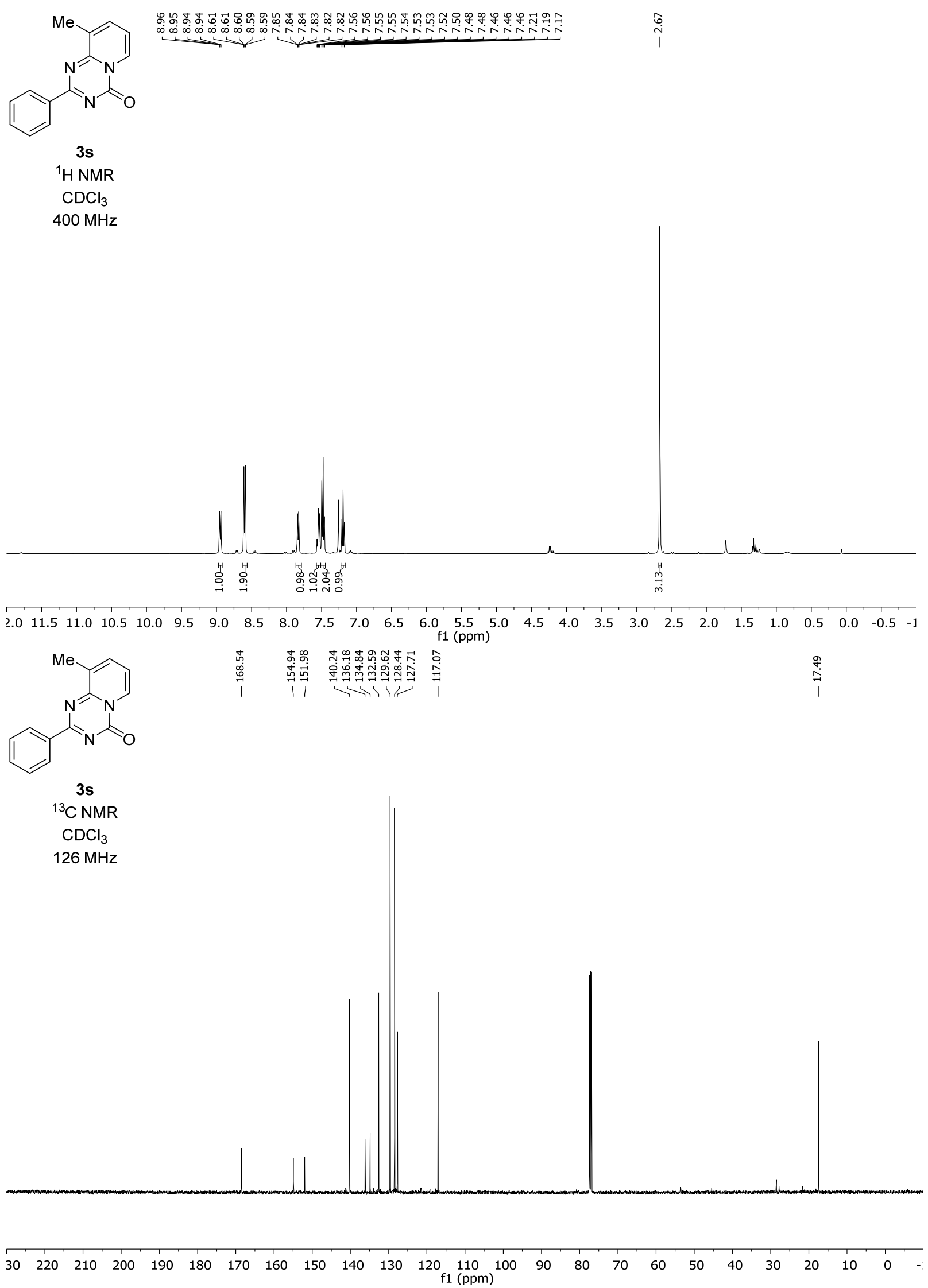


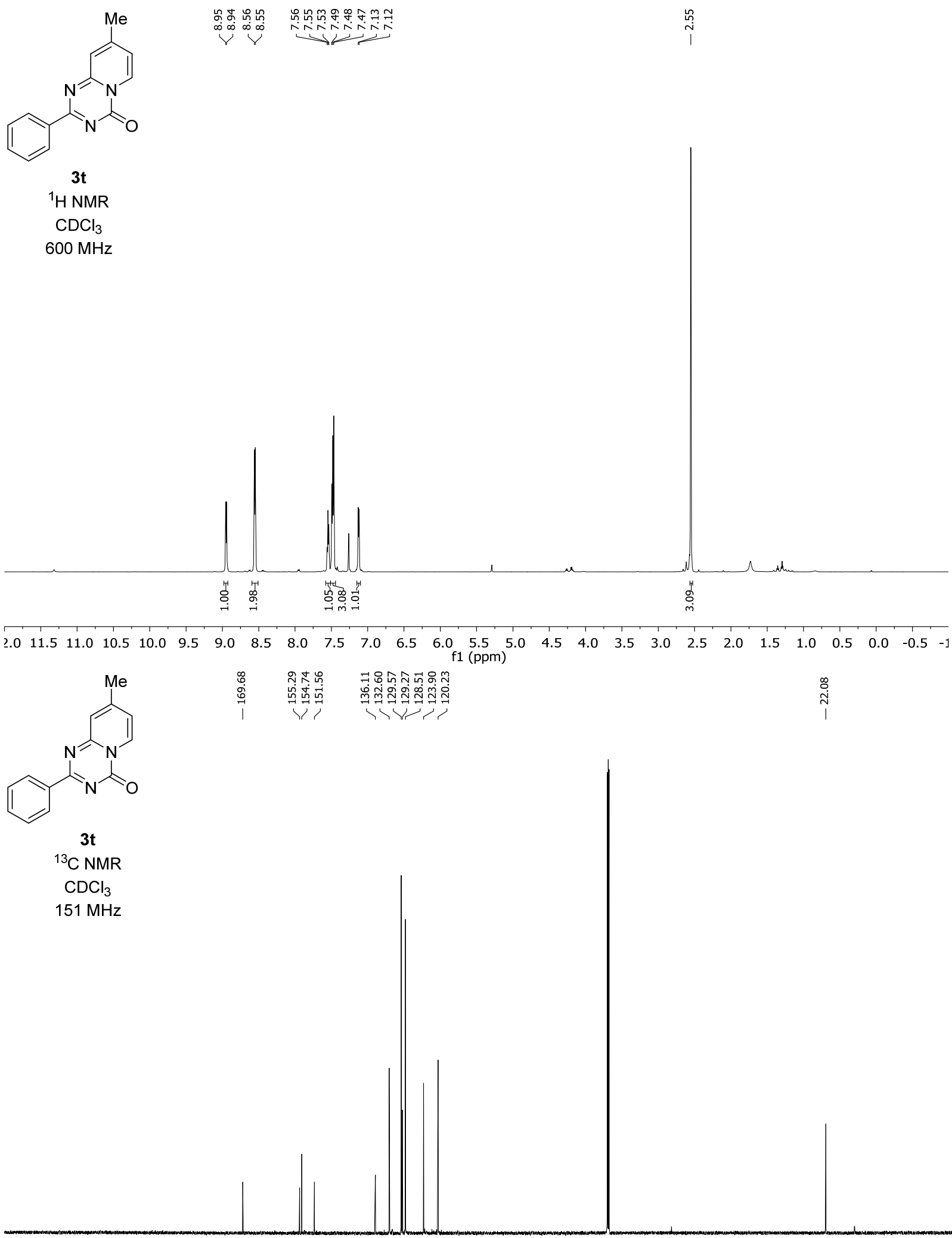

$\begin{array}{lllllllllllllllllllllllllllll}30 & 220 & 210 & 200 & 190 & 180 & 170 & 160 & 150 & 140 & 130 & 120 & 110 & 100 & 90 & 80 & 70 & 60 & 50 & 40 & 30 & 20 & 10 & 0 & -\end{array}$ 

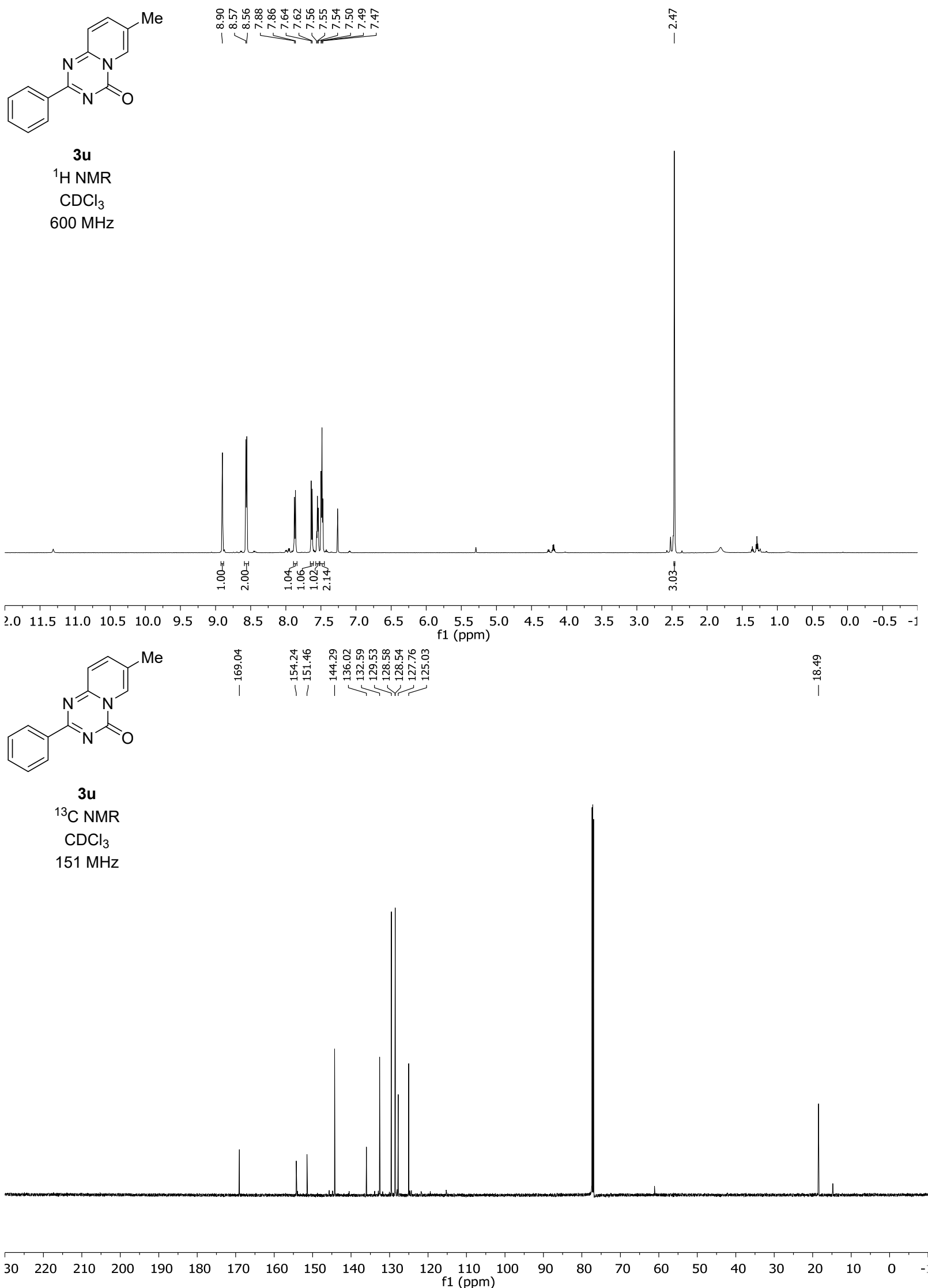


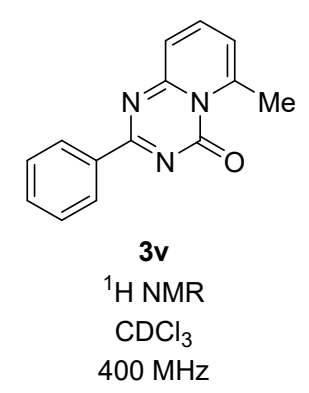

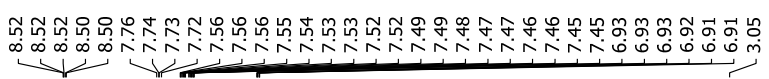

$400 \mathrm{MHz}$

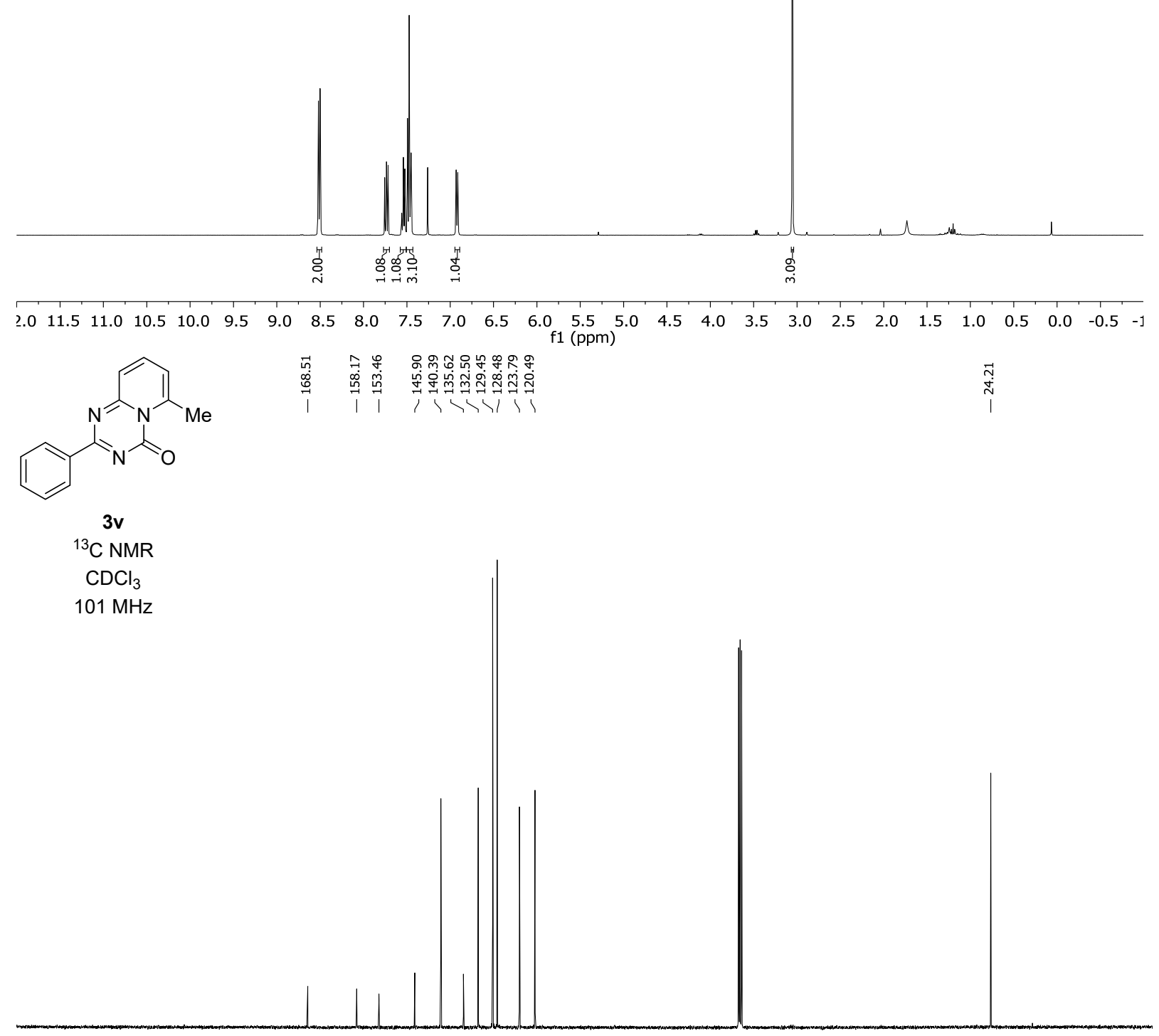

$\begin{array}{llllllllllllllllllllllllllll}30 & 220 & 210 & 200 & 190 & 180 & 170 & 160 & 150 & 140 & 130 & 120 & \begin{array}{l}110 \\ \mathrm{f} 1\end{array}(\mathrm{ppm}) & 100 & 90 & 80 & 70 & 60 & 50 & 40 & 30 & 20 & 10 & 0 & -\end{array}$ 

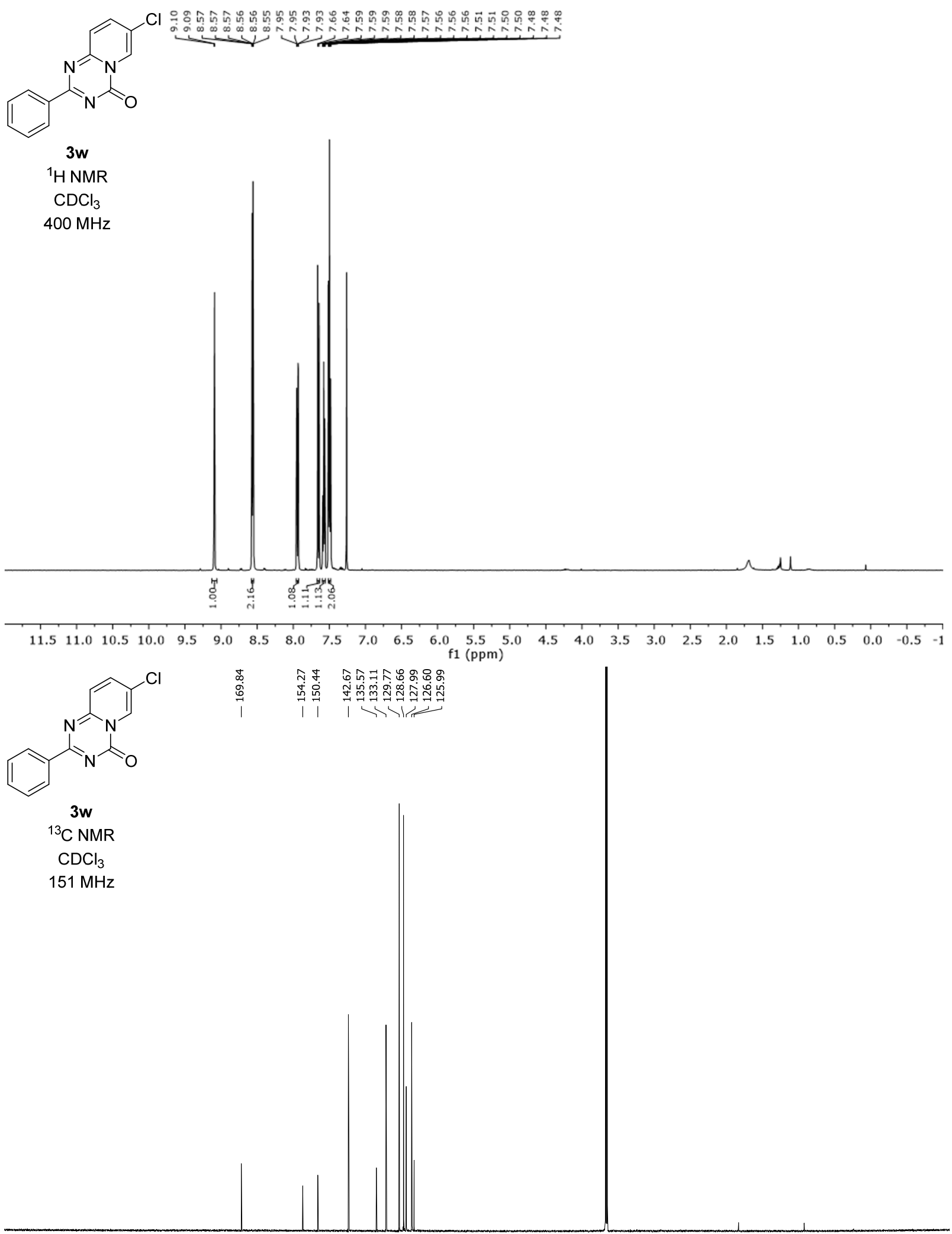

30

$\begin{array}{llllll}30 & 220 & 210 & 200 & 190 & 180\end{array}$

170160

$150 \quad 140$

130

$120 \quad 110 \quad 100$

9080

8070 

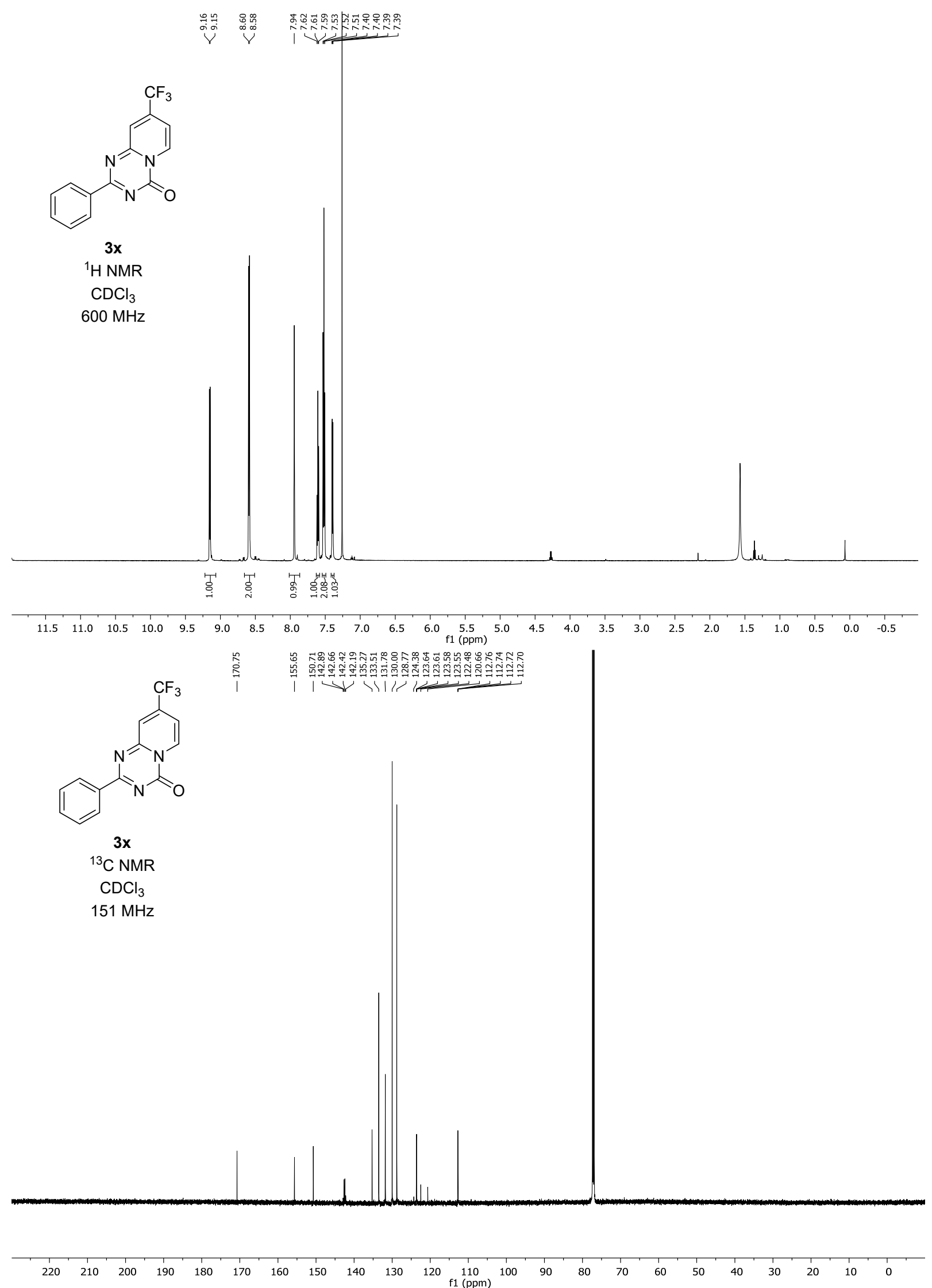


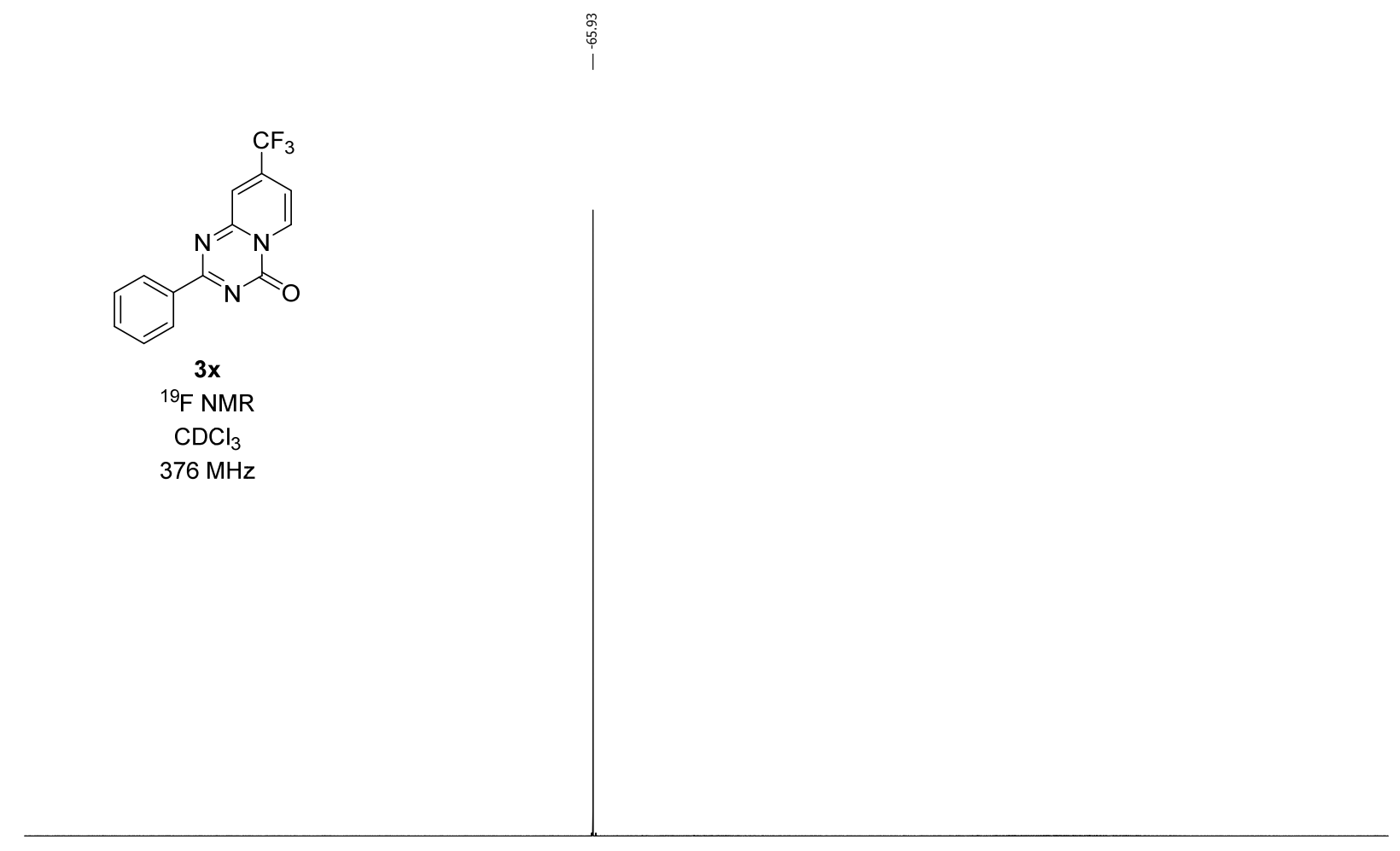

\begin{tabular}{lllllllllllllllllllllllllllll}
\hline 0 & 20 & 10 & 0 & -10 & -20 & -30 & -40 & -50 & -60 & -70 & -80 & -90 & -100 & -110 & -120 & -130 & -140 & -150 & -160 & -170 & -180 & -190 & -2
\end{tabular} 


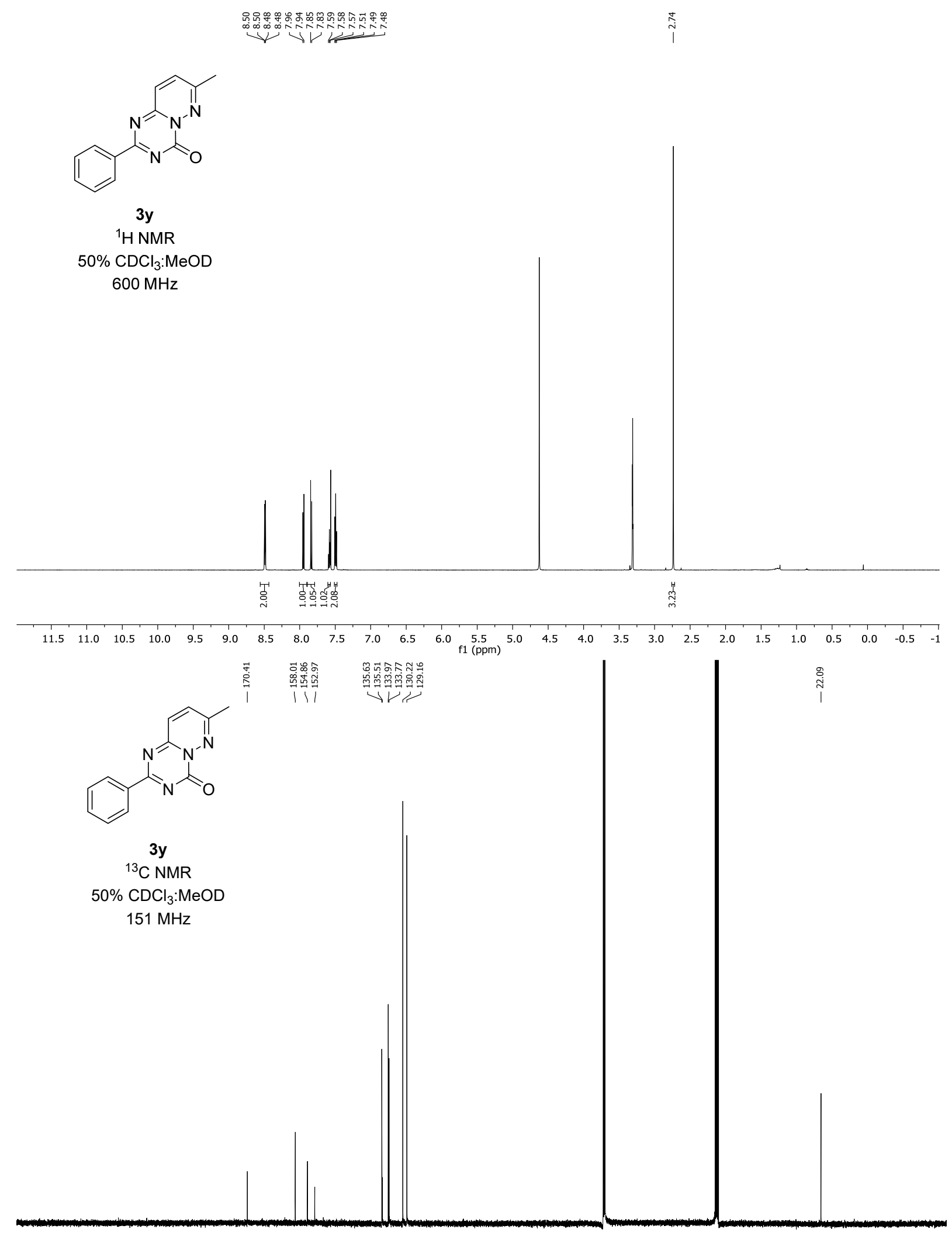

\begin{tabular}{llllllllllllllllllllllllllll}
\hline 30 & 220 & 210 & 200 & 190 & 180 & 170 & 160 & 150 & 140 & 130 & 120 & 110 & 100 & 90 & 80 & 70 & 60 & 50 & 40 & 30 & 20 & 10 & 0 & -1
\end{tabular} 

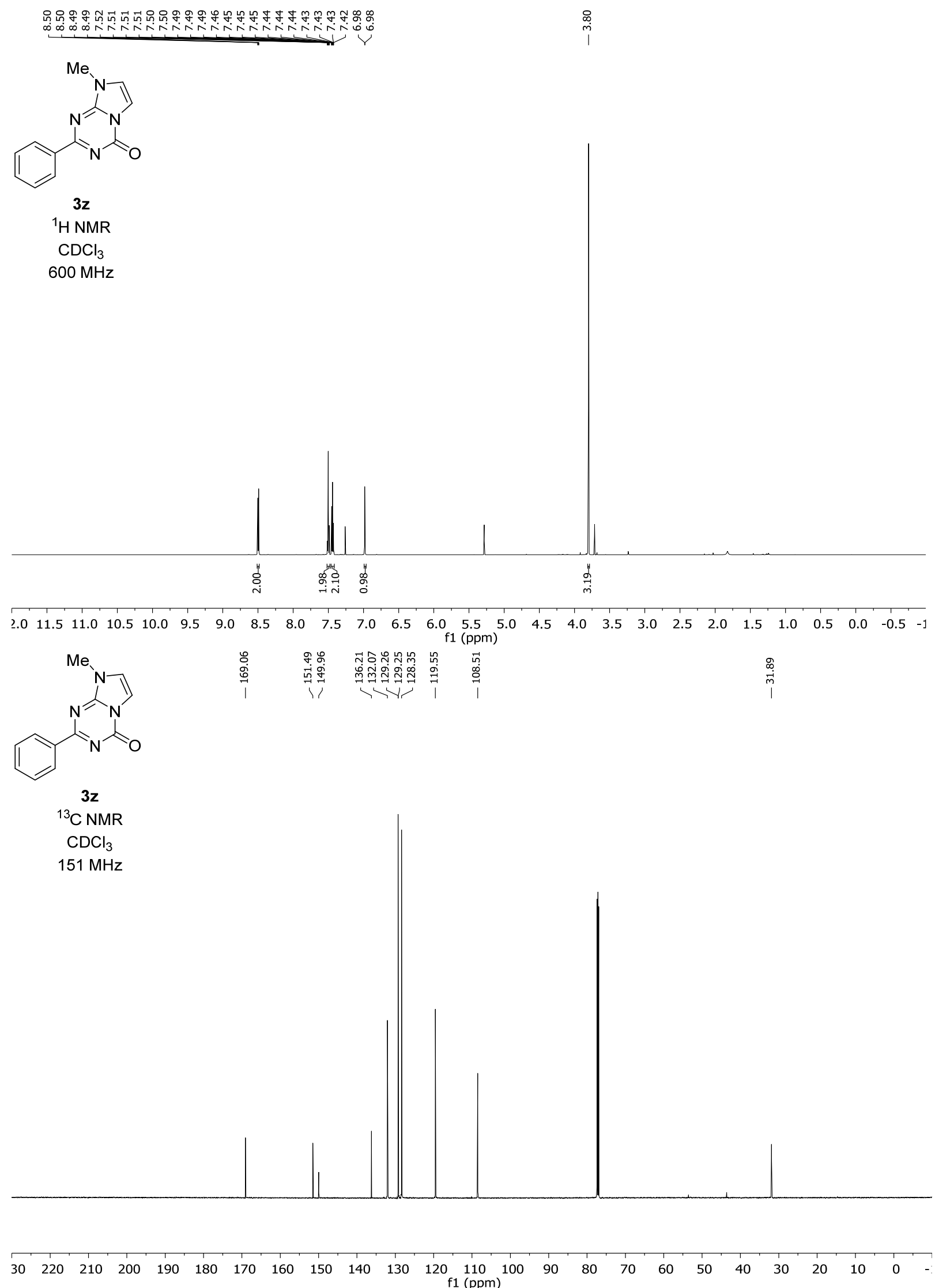


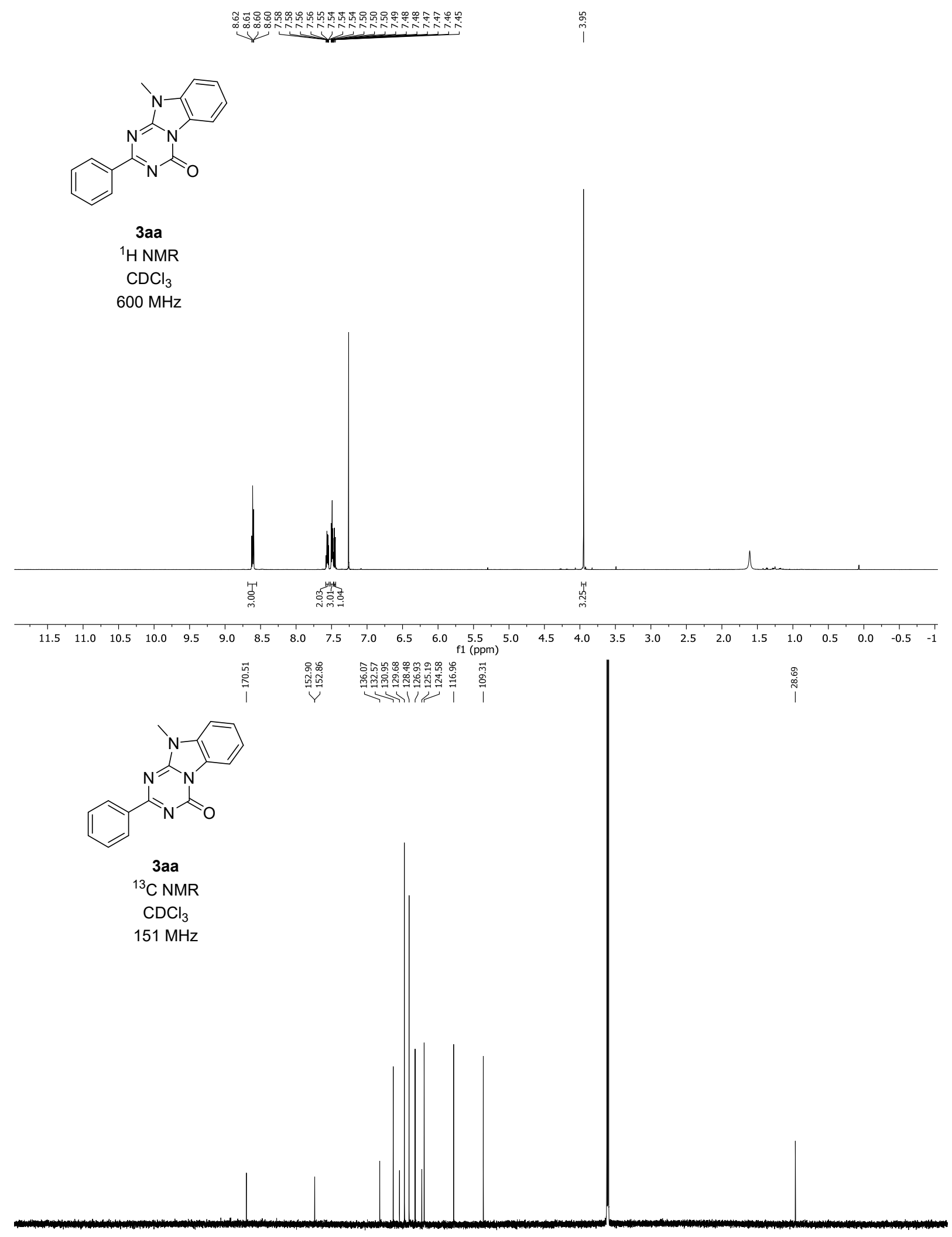

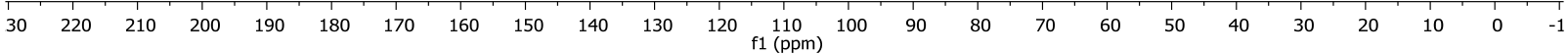



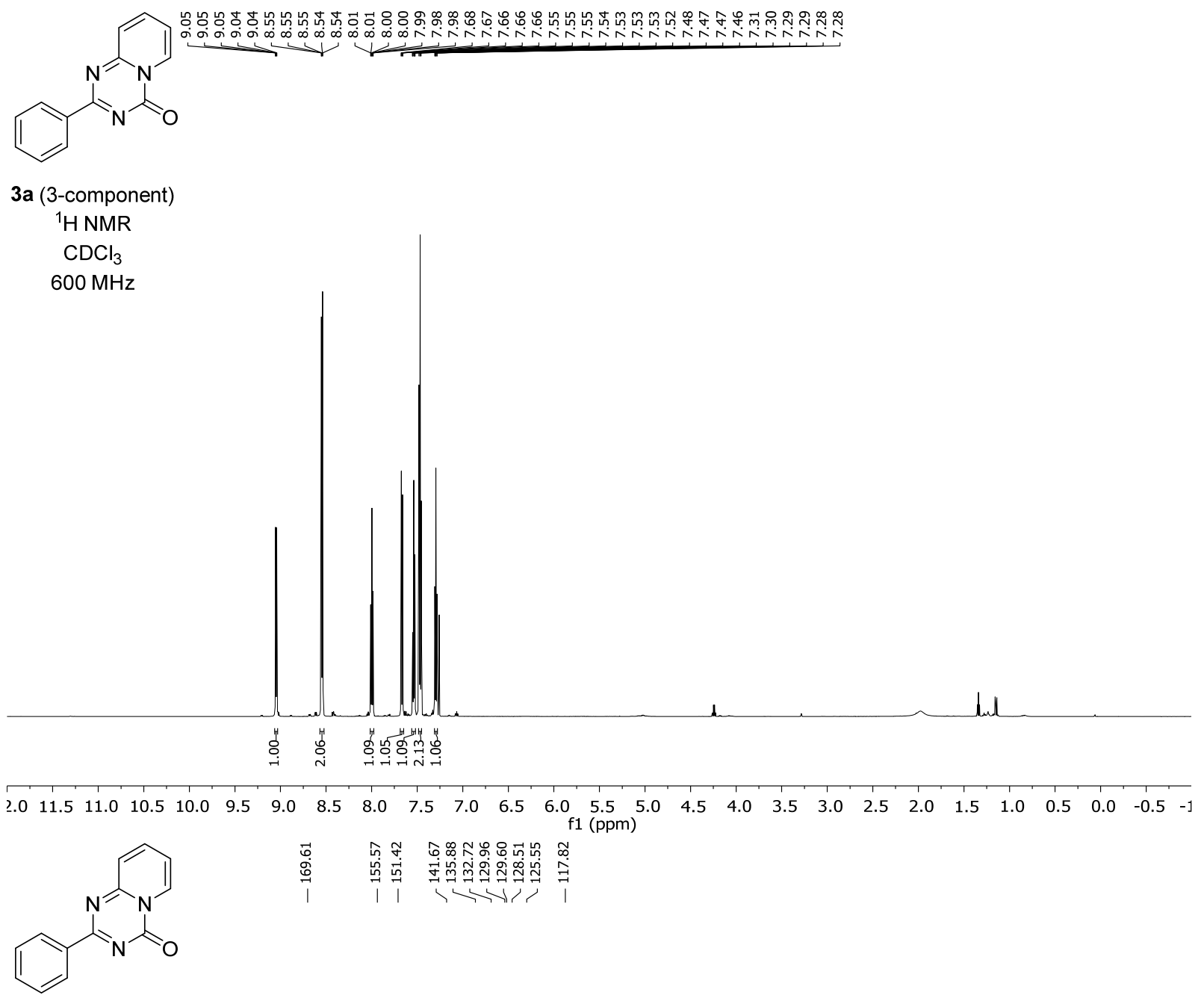

3a (3-component)

${ }^{13} \mathrm{C}$ NMR

$\mathrm{CDCl}_{3}$

$151 \mathrm{MHz}$

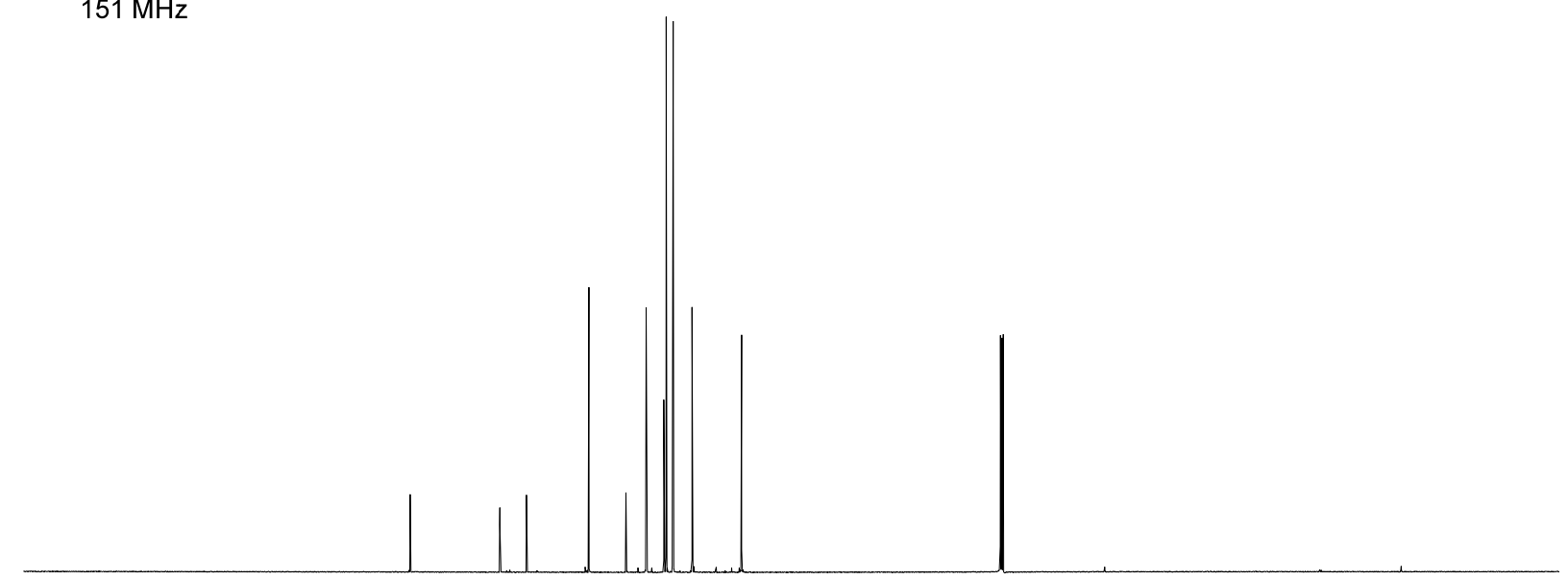

30

$220 \quad 210 \quad 200 \quad 190 \quad 180-170 \quad 160$

150140

$20 \quad 110 \quad 100$

$\begin{array}{llll}90 & 80 & 70 & 60\end{array}$

50

$\begin{array}{lllll}40 & 30 & 20 & 10 & 0\end{array}$ 


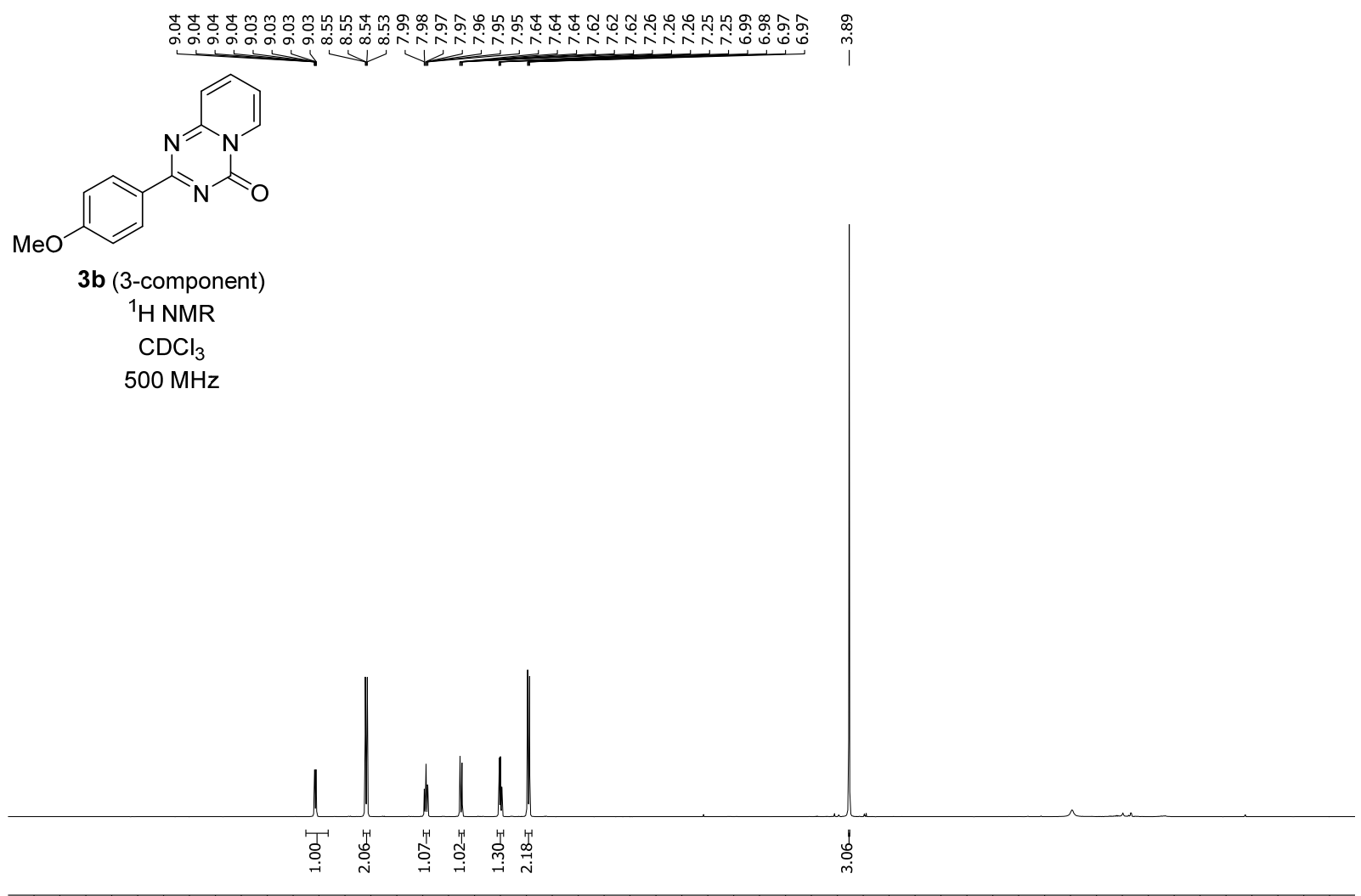

$\begin{array}{llllllllllllllllllllllllll}11.5 & 11.0 & 10.5 & 10.0 & 9.5 & 9.0 & 8.5 & 8.0 & 7.5 & 7.0 & 6.5 & 6.0 & \begin{array}{c}5.5 \\ \mathrm{f} 1(\mathrm{ppm})\end{array} \mathbf{5 . 0} & 4.5 & 4.0 & 3.5 & 3.0 & 2.5 & 2.0 & 1.5 & 1.0 & 0.5 & 0.0 & -0.5 & -1\end{array}$<smiles>COc1ccc(-c2nc(=O)n3ccccc3n2)cc1</smiles>

กั่

|

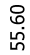

${ }^{13} \mathrm{C}$ NMR

$\mathrm{CDCl}_{3}$

$151 \mathrm{MHz}$

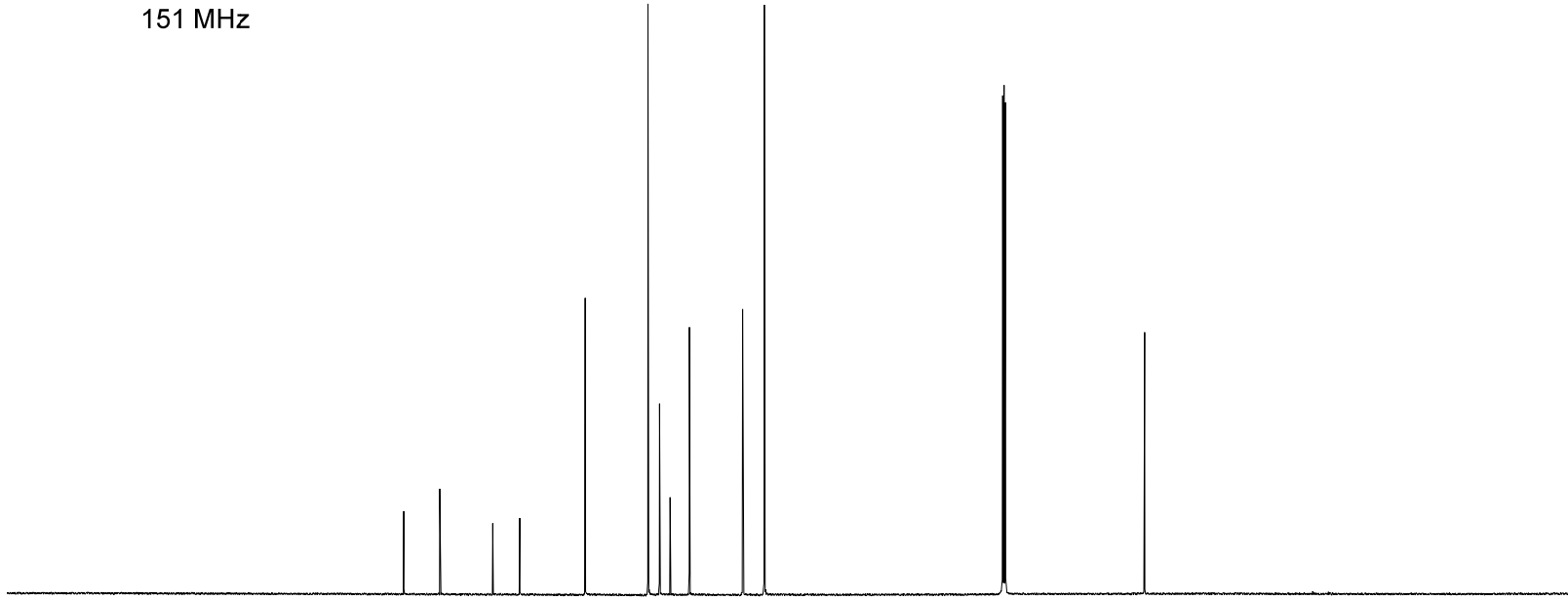

$\begin{array}{lllllllllllllllllllllllllll}30 & 220 & 210 & 200 & 190 & 180 & 170 & 160 & 150 & 140 & 130 & 120 & \begin{array}{l}110 \\ \mathrm{f} 1\end{array}(\mathrm{ppm}) & 100 & 90 & 80 & 70 & 60 & 50 & 40 & 30 & 20 & 10 & 0 & -\end{array}$ 

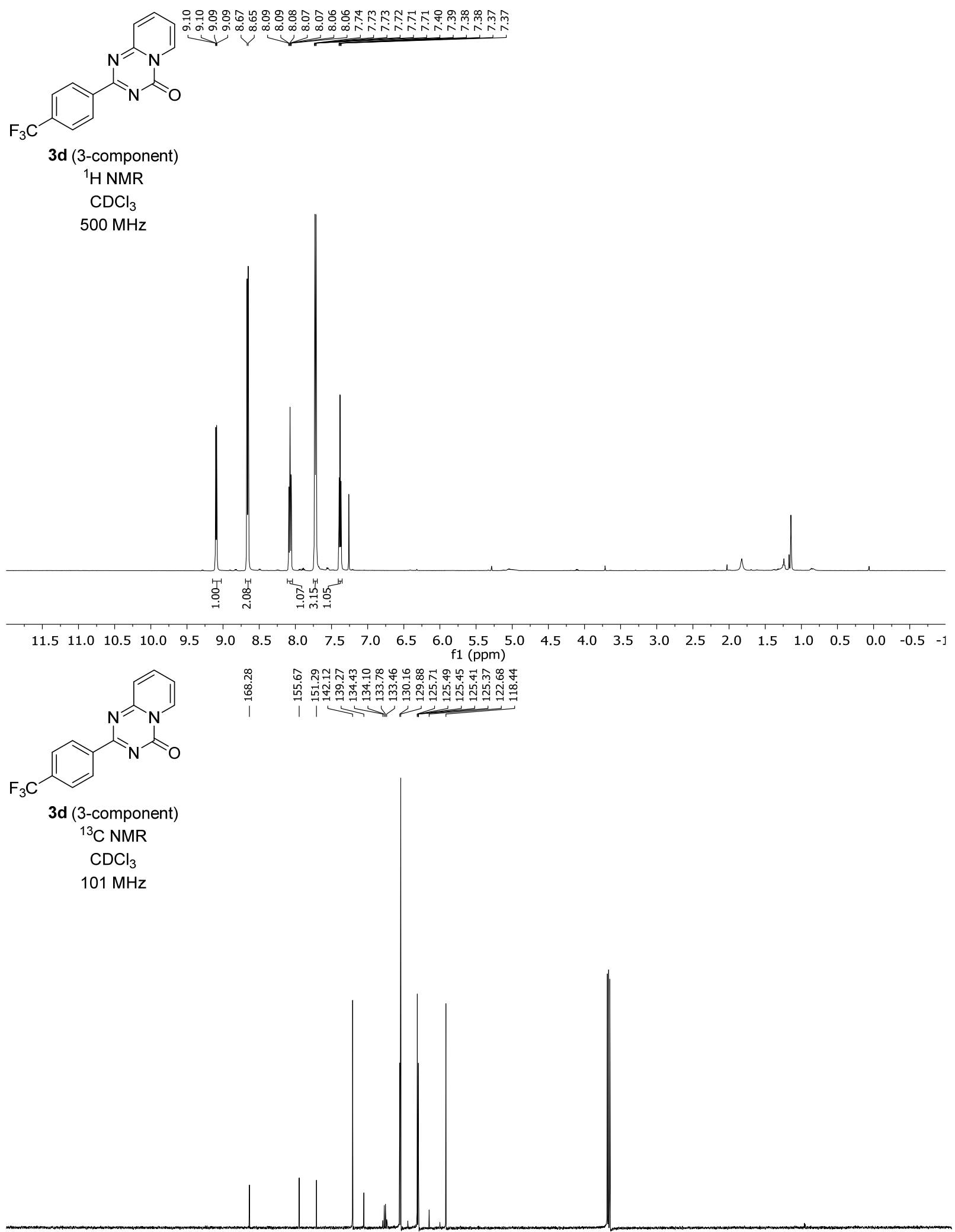

30

$\begin{array}{llllll}220 & 210 & 200 & 190 & 180\end{array}$

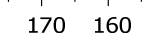

150140

130

$20 \quad 110 \quad 100$

$90 \quad 80 \quad 70$ 


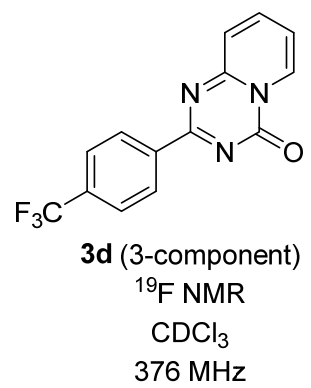

ผू

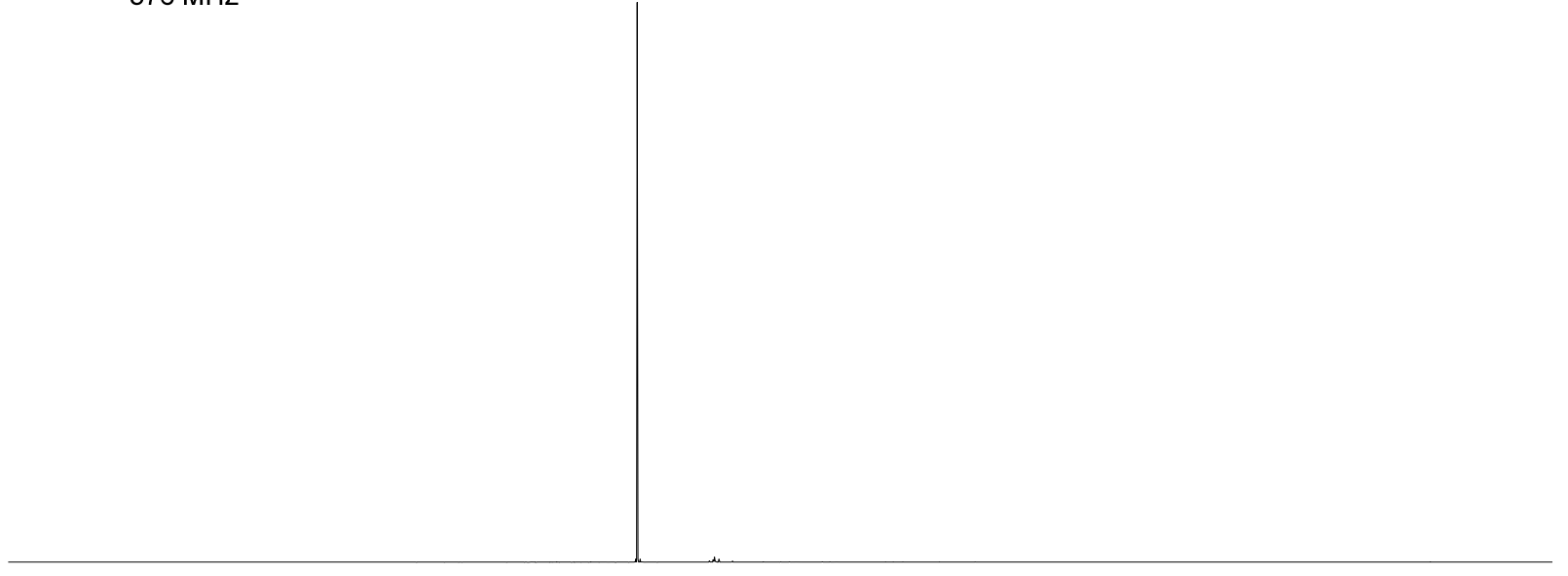

$\begin{array}{llllllllllllllllllllllllllll}30 & 20 & 10 & 0 & -10 & -20 & -30 & -40 & -50 & -60 & -70 & -80 & -90 & -100 & -110 & -120 & -130 & -140 & -150 & -160 & -170 & -180 & -190 & -20 \mathrm{C}\end{array}$ 


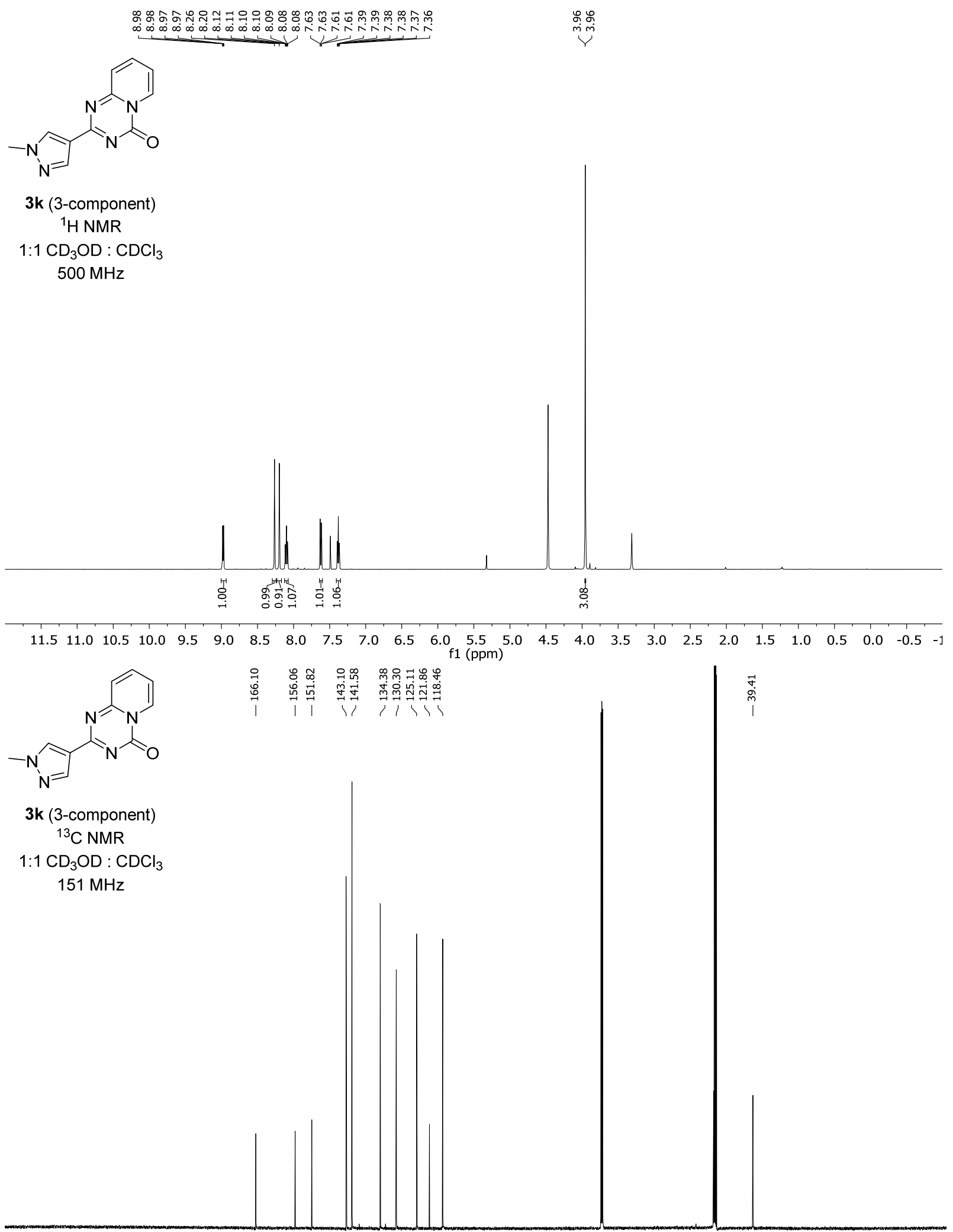

$\begin{array}{lllllllllllllllllllllllllll}30 & 220 & 210 & 200 & 190 & 180 & 170 & 160 & 150 & 140 & 130 & 120 & 110 & 100 & 90 & 80 & 70 & 60 & 50 & 40 & 30 & 20 & 10 & 0 & -\end{array}$ 


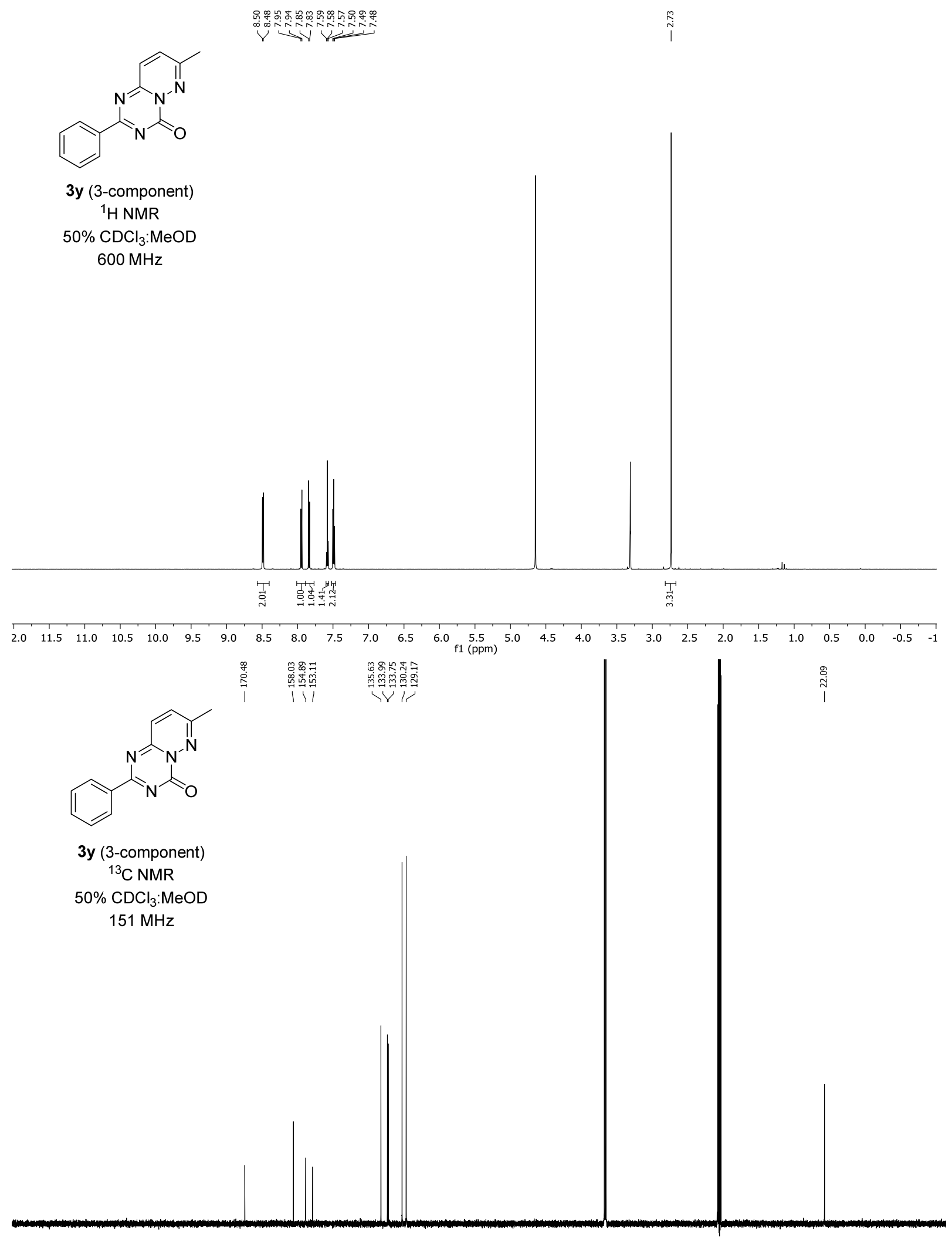

$\begin{array}{llllllllllllllllllllllllllllllllllll}30 & 220 & 210 & 200 & 190 & 180 & 170 & 160 & 150 & 140 & 130 & 120 & 110 & 100 & 90 & 80 & 70 & 60 & 50 & 40 & 30 & 20 & 10 & 0\end{array}$ 

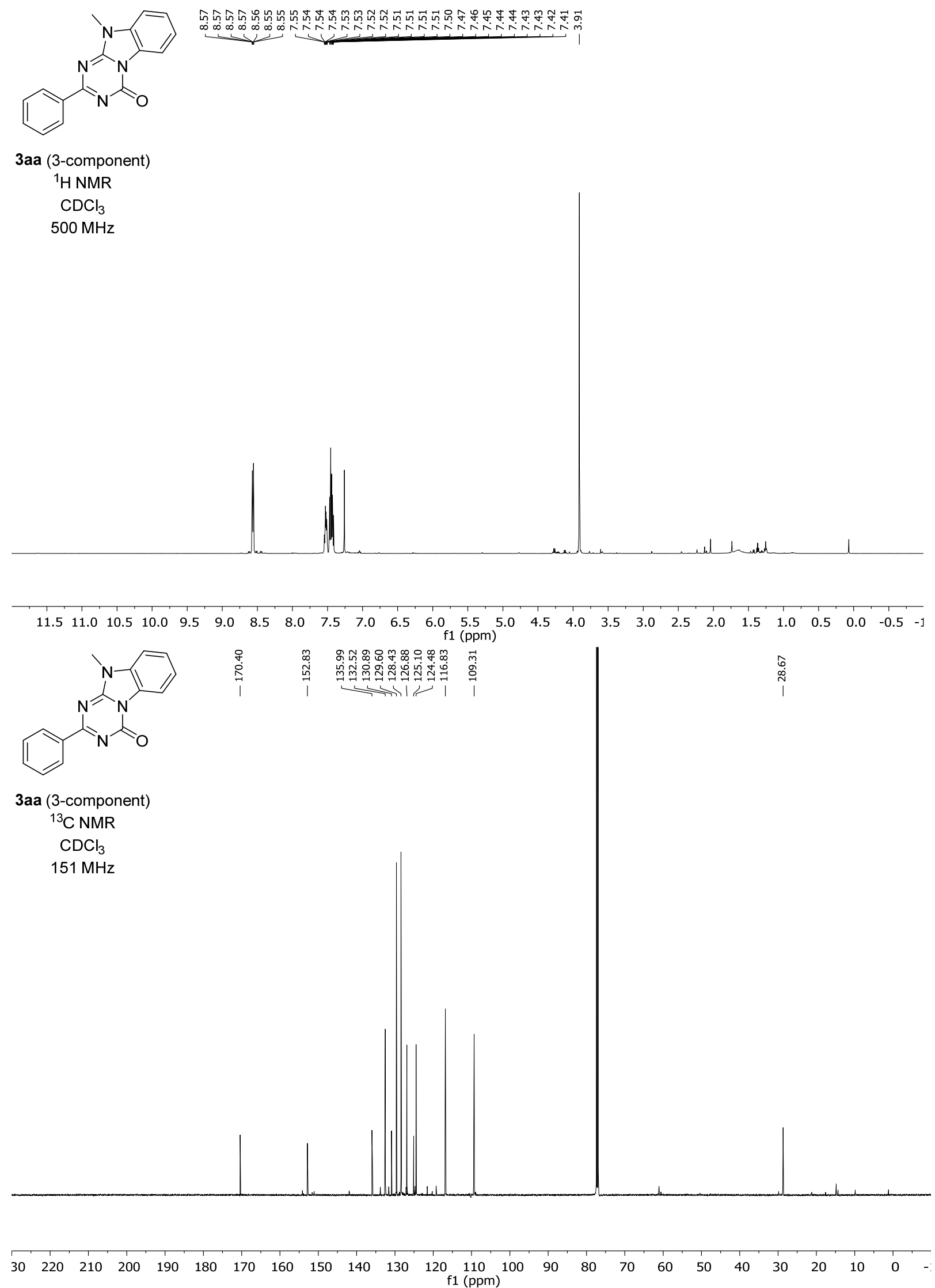

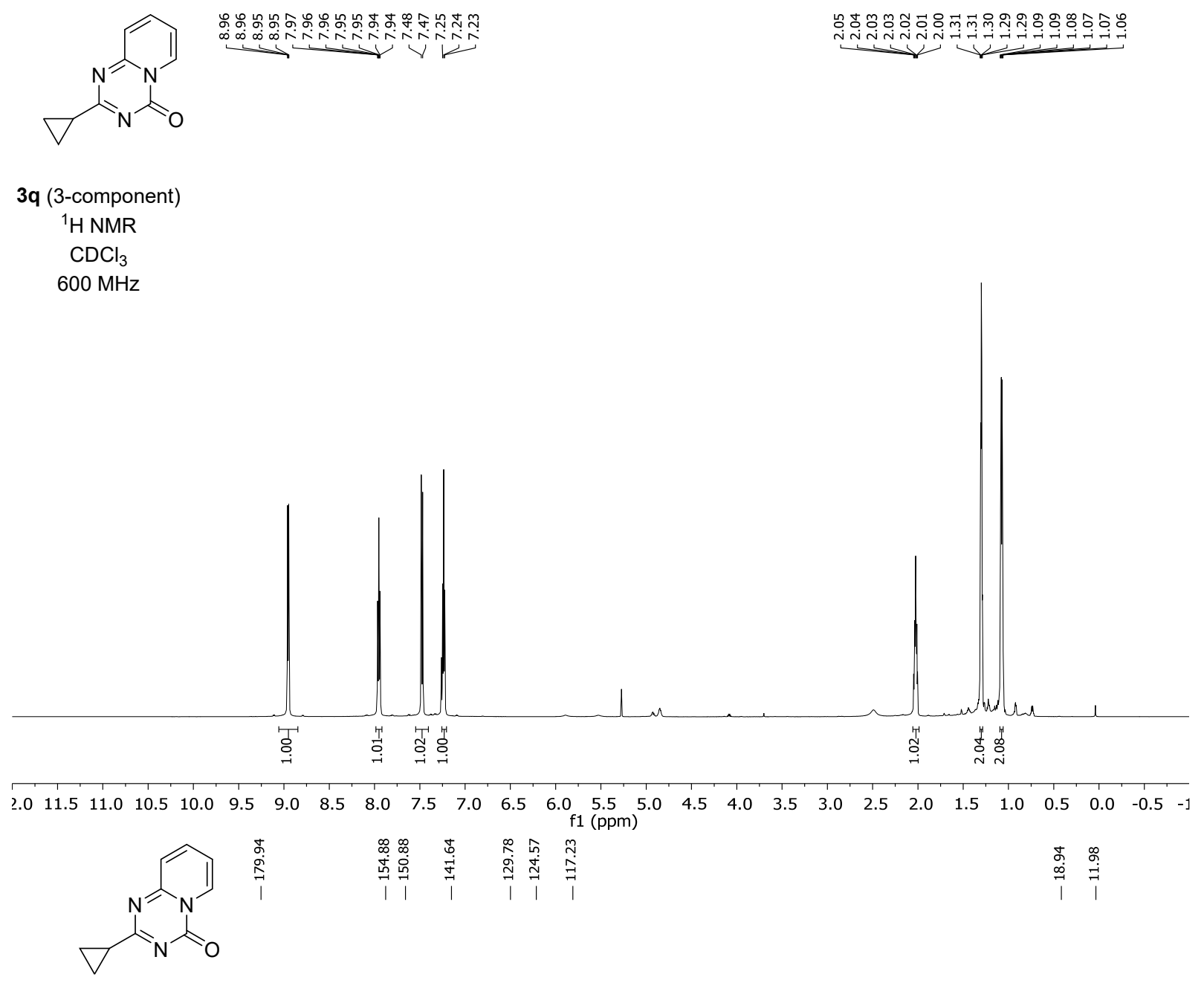

3q (3-component)

${ }^{13} \mathrm{C}$ NMR

$\mathrm{CDCl}_{3}$

$151 \mathrm{MHz}$

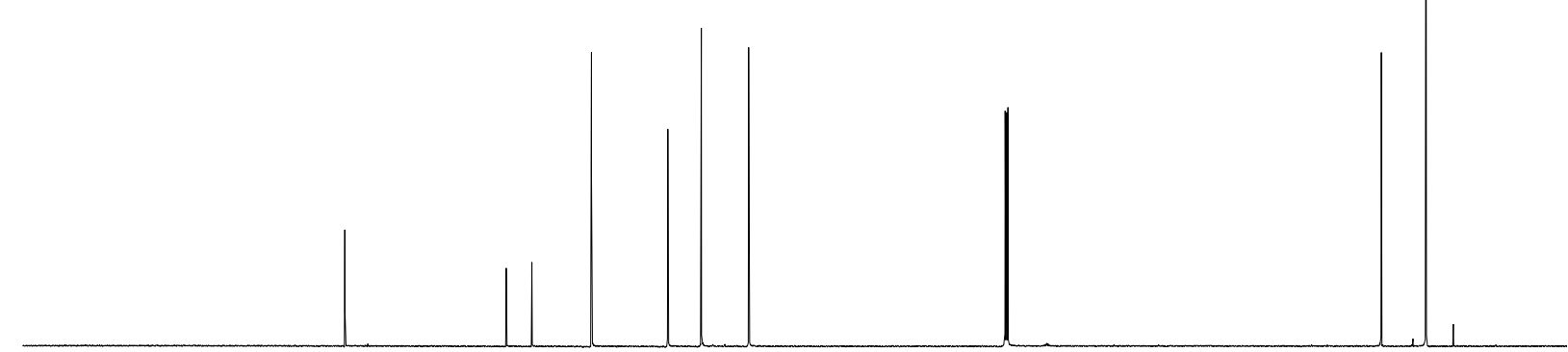

$\begin{array}{lllllllllllllllllllllllll}30 & 220 & 210 & 200 & 190 & 180 & 170 & 160 & 150 & 140 & 130 & 120 & \begin{array}{c}110 \\ \mathrm{f} 1(\mathrm{ppm})\end{array} & 100 & 90 & 80 & 70 & 60 & 50 & 40 & 30 & 20 & 10 & 0 & -\end{array}$ 


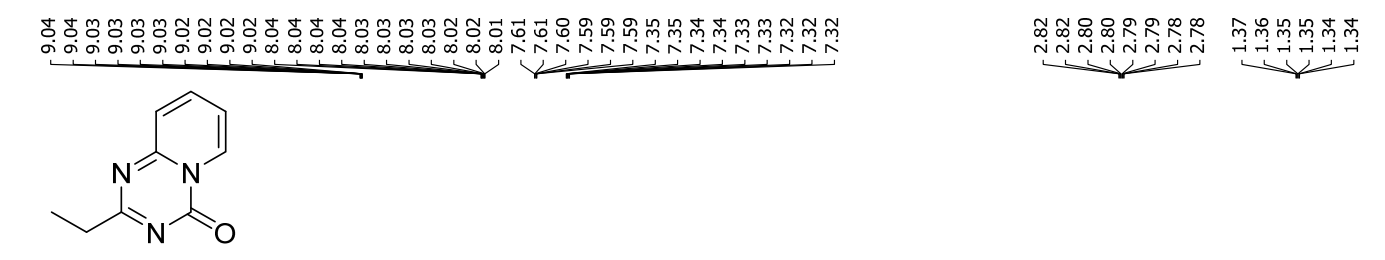

3ab (3-component)

${ }^{1} \mathrm{H}$ NMR

$\mathrm{CDCl}_{3}$

$600 \mathrm{MHz}$

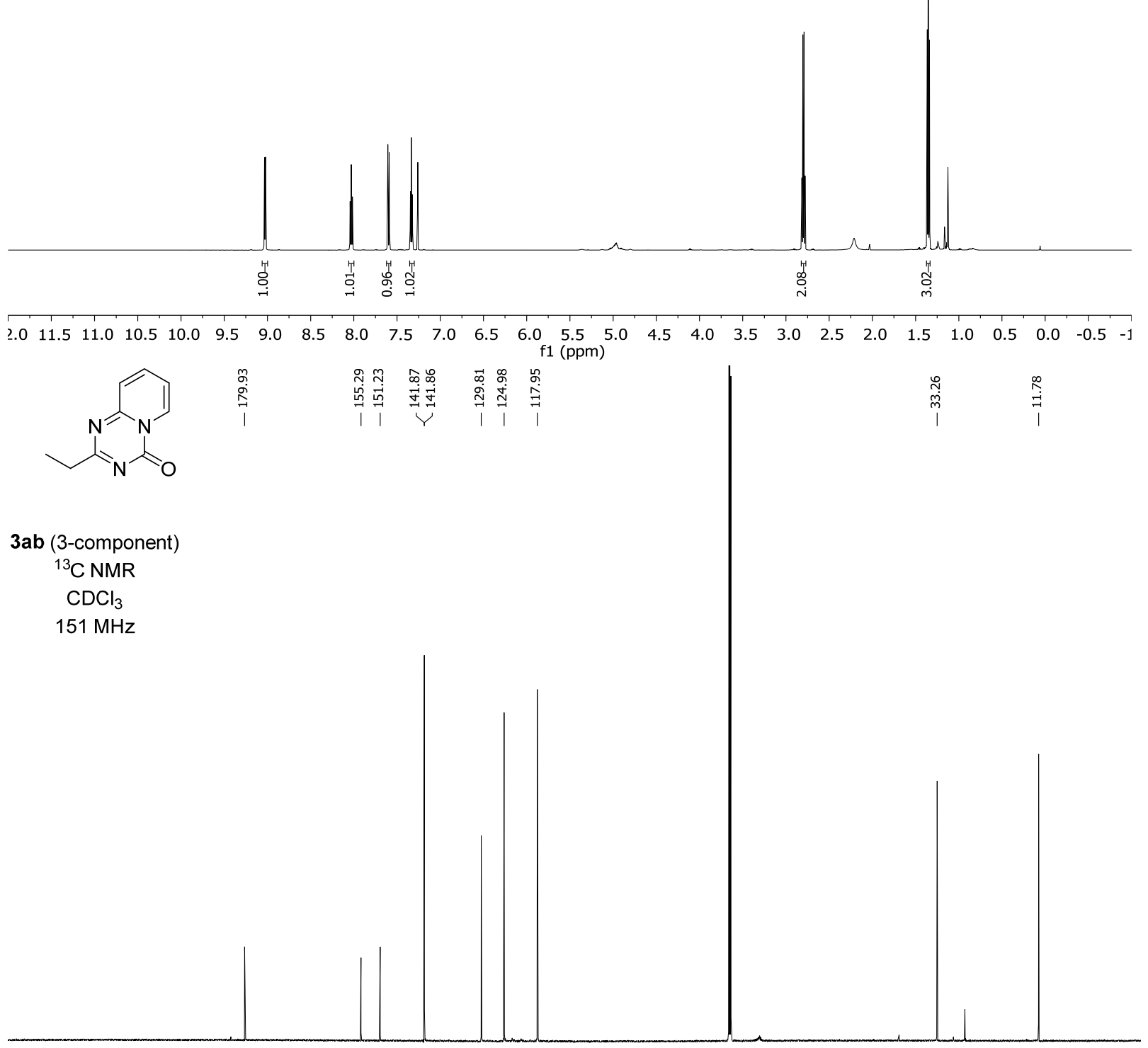

$\begin{array}{llllllllllllllllllllllll}220 & 210 & 200 & 190 & 180 & 170 & 160 & 150 & 140 & 130 & 120 & \left.\begin{array}{l}110 \\ \mathrm{f} 1(\mathrm{ppm})\end{array}\right) & 100 & 90 & 80 & 70 & 60 & 50 & 40 & 30 & 20 & 10 & 0 & -\end{array}$ 

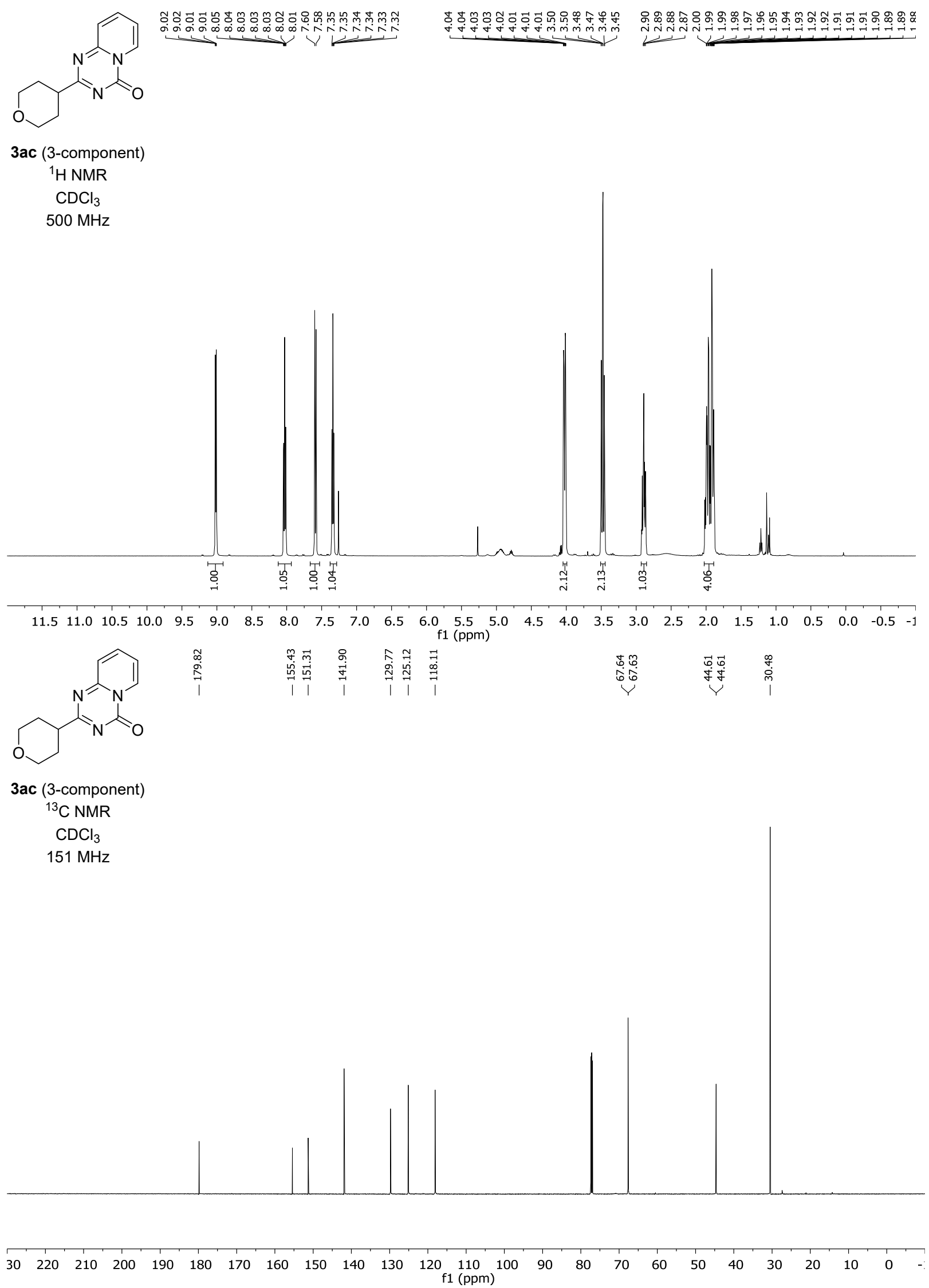

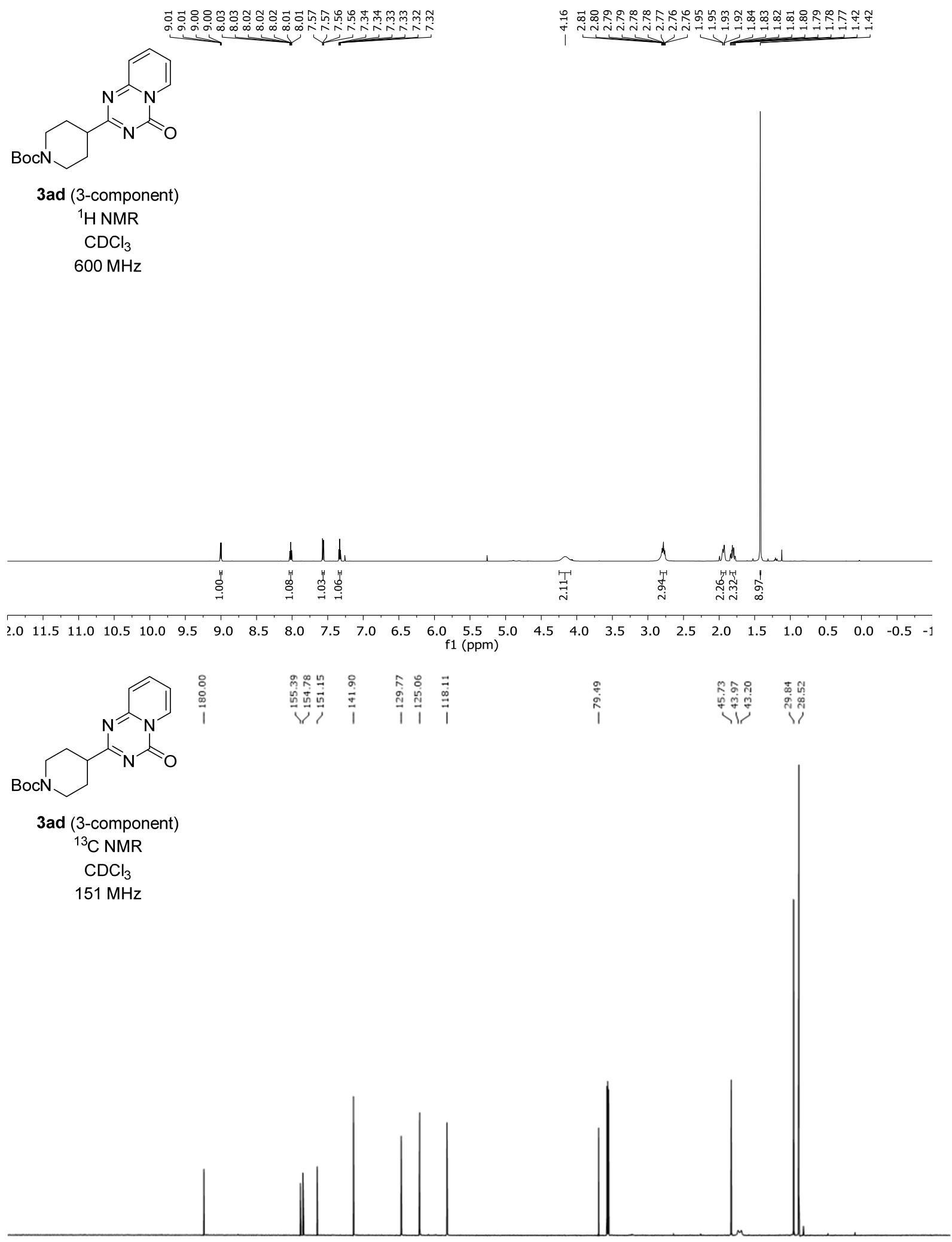

$\begin{array}{llllllllllllllllllllllllllllllll}30 & 220 & 210 & 200 & 190 & 180 & 170 & 160 & 150 & 140 & 130 & 120 & 110 & 100 & 90 & 80 & 70 & 60 & 50 & 40 & 30 & 20 & 10 & 0 & -1(\mathrm{ppm})\end{array}$ 

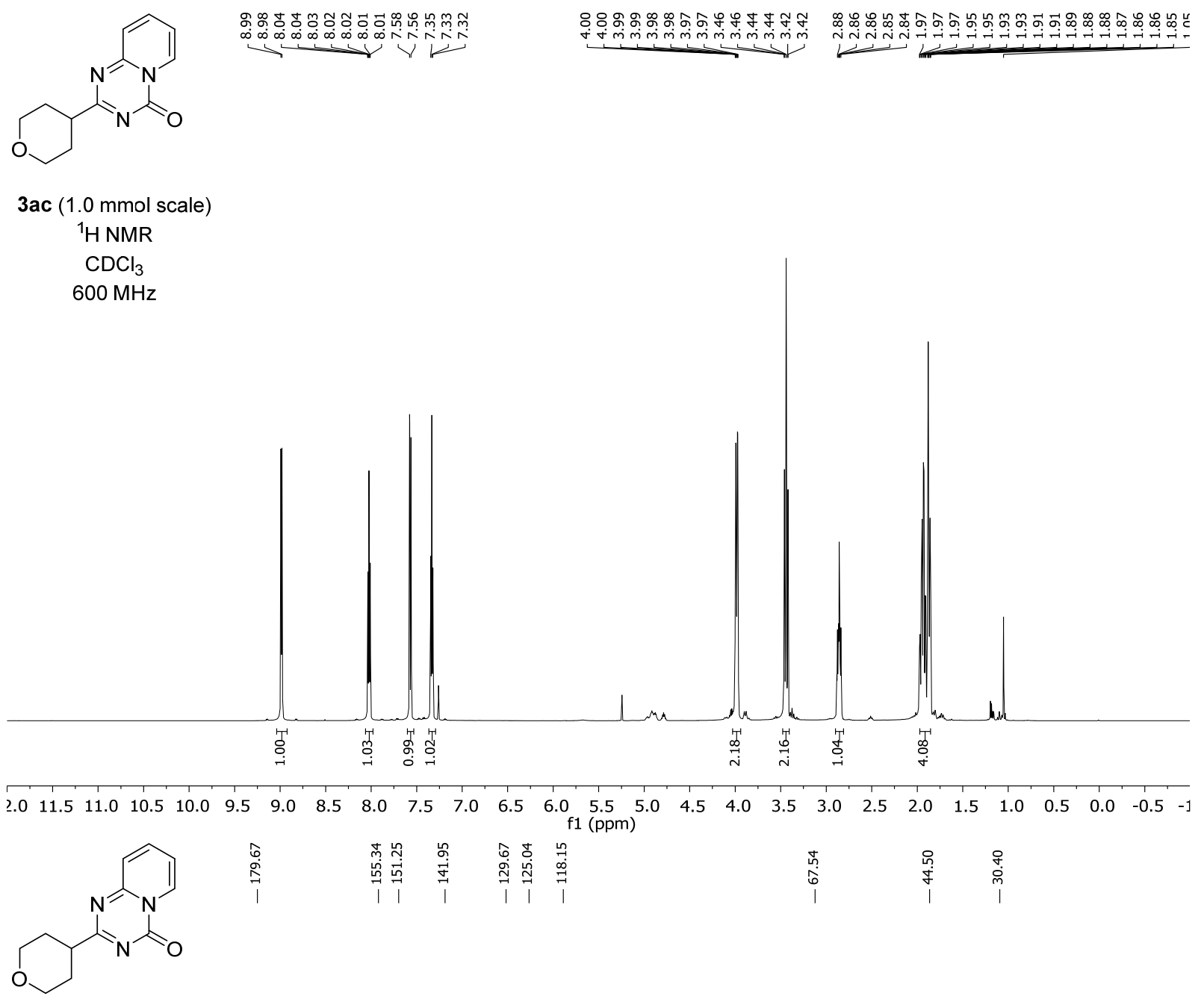

\footnotetext{
${ }^{13} \mathrm{C}$ NMR

$\mathrm{CDCl}_{3}$

$151 \mathrm{MHz}$
}

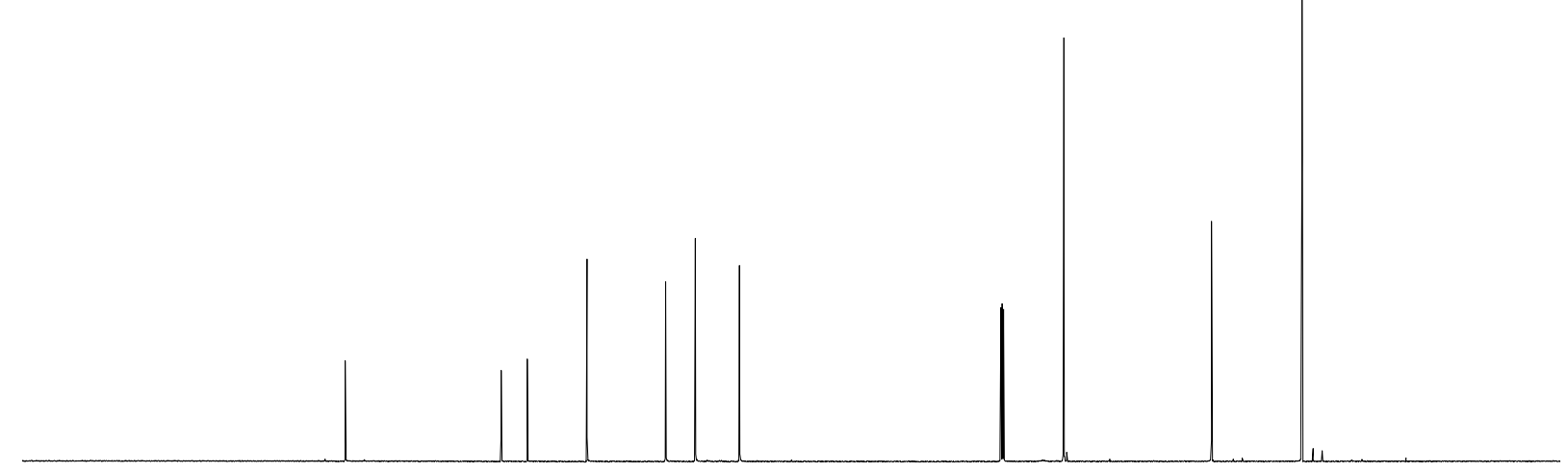

$30 \quad 220$

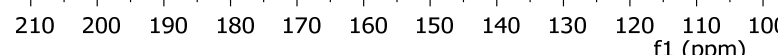

1 (ppm) 


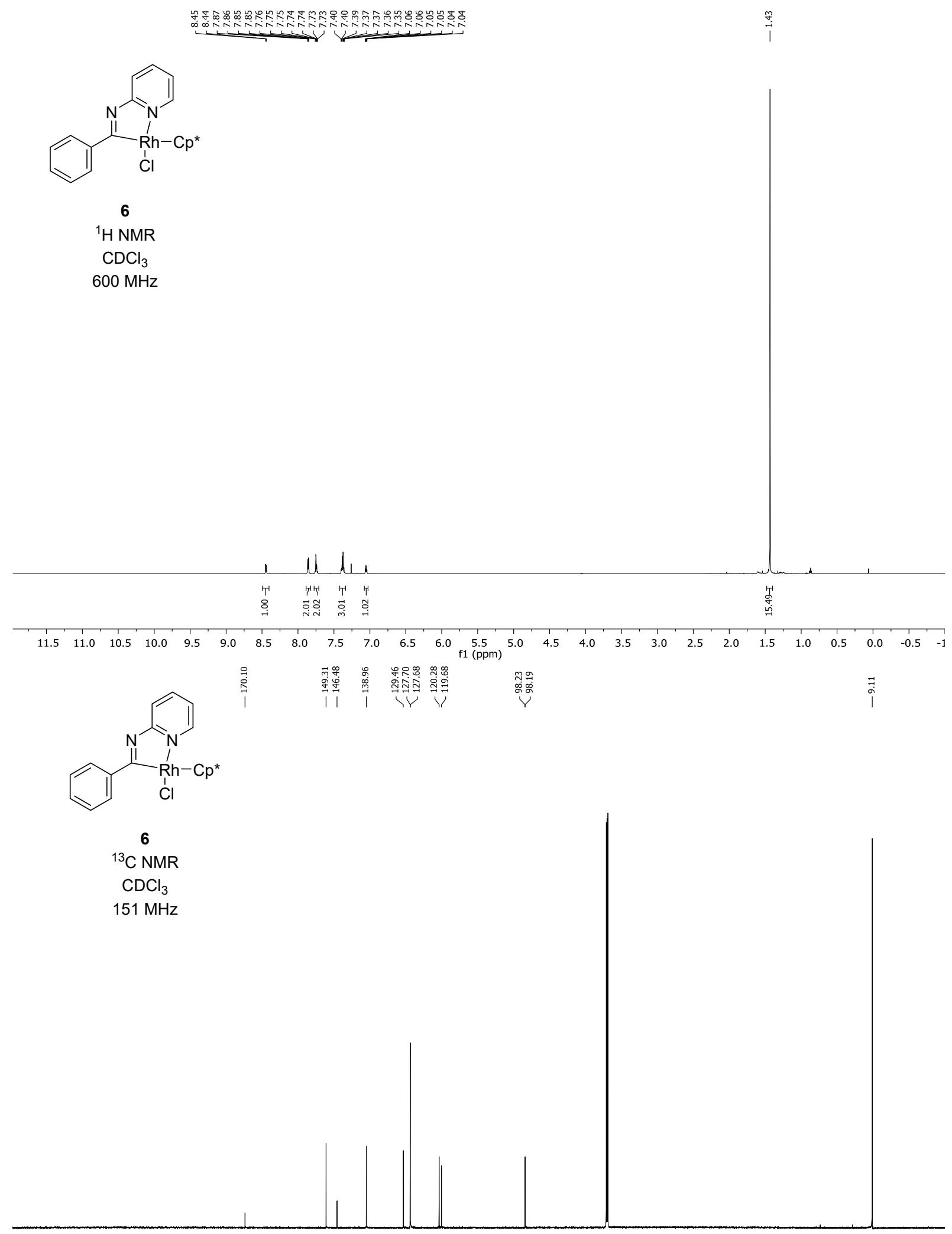

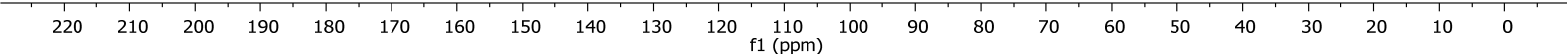


<smiles>CCOC(=O)N/C(=N\c1ccccn1)c1ccccc1Br</smiles>

7

${ }^{1} \mathrm{H}$ NMR

$\mathrm{CDCl}_{3}$

$400 \mathrm{MHz}$

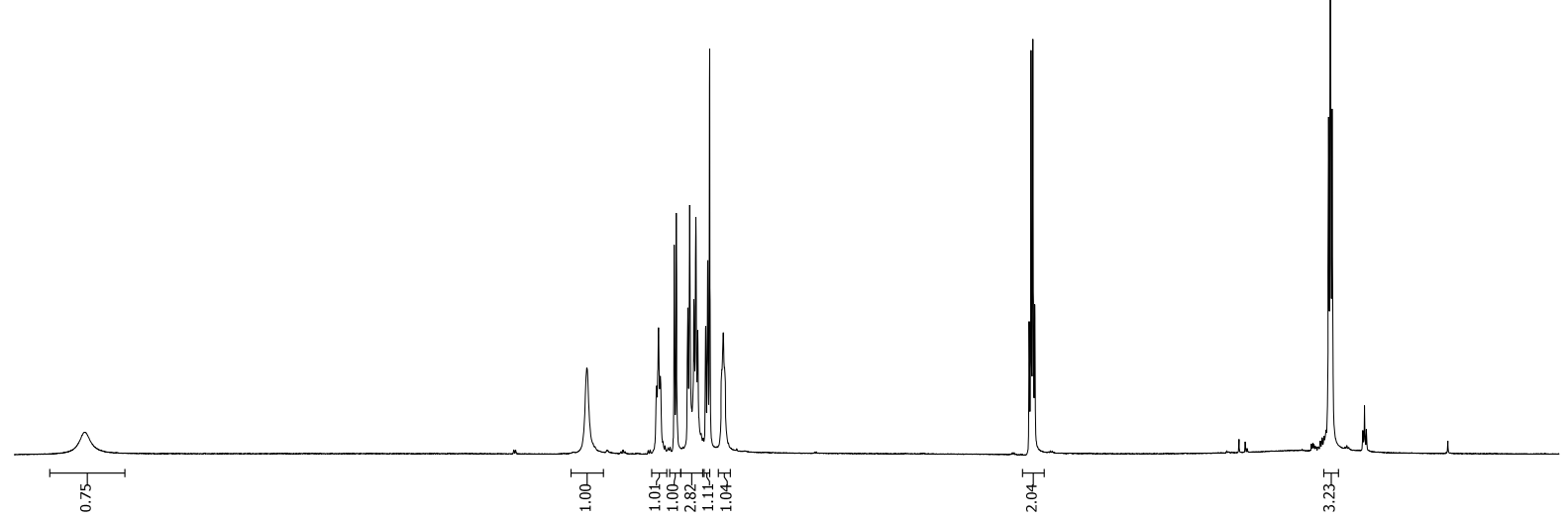

$\begin{array}{lllllllllllllllllllllllllllllllllllllllllllllll}4.0 & 13.5 & 13.0 & 12.5 & 12.0 & 11.5 & 11.0 & 10.5 & 10.0 & 9.5 & 9.0 & 8.5 & 8.0 & 7.5 & 7.0 & 6.5 & 6.0 & 5.5 & 5.0 & 4.5 & 4.0 & 3.5 & 3.0 & 2.5 & 2.0 & 1.5 & 1.0 & 0.5 & 0.0 & -0.5 & -1\end{array}$

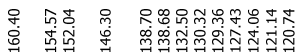

| । |<smiles>CCOC(=O)N/C(=N\c1ccccn1)c1ccccc1Br</smiles>

7

${ }^{13} \mathrm{C}$ NMR

$\mathrm{CDCl}_{3}$

$101 \mathrm{MHz}$

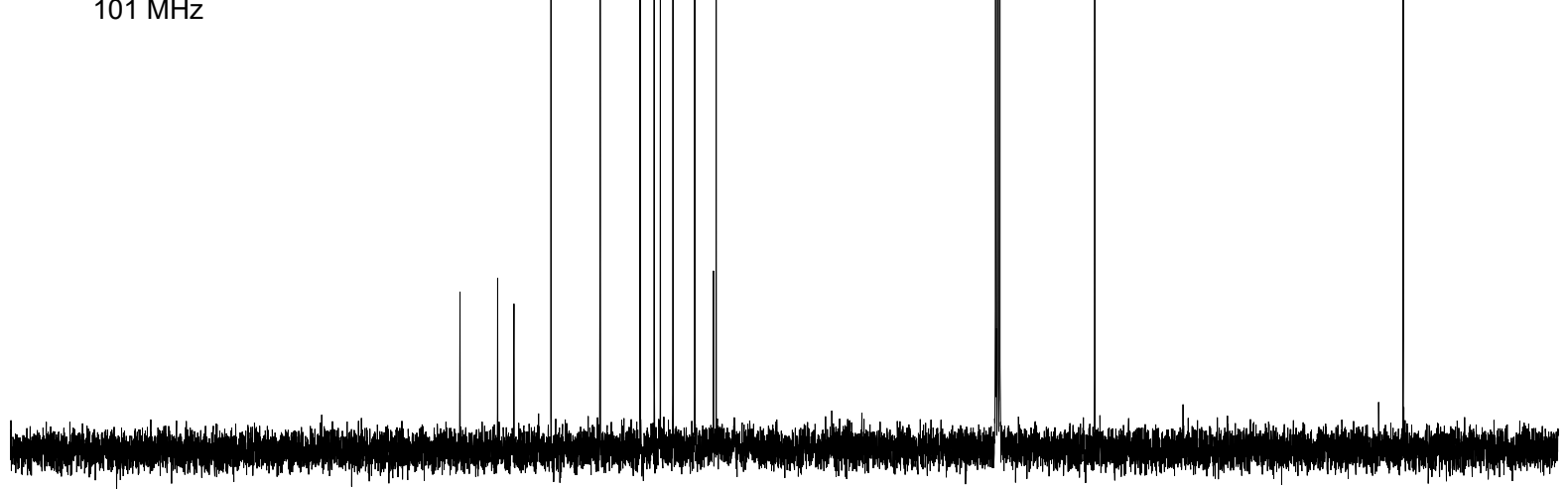

$\begin{array}{lllllllllllllllllllllllll}30 & 220 & 210 & 200 & 190 & 180 & 170 & 160 & 150 & 140 & 130 & 120 & 110 & 100 & 90 & 80 & 70 & 60 & 50 & 40 & 30 & 20 & 10 & 0\end{array}$ 


\section{X-ray crystallographic data}

\section{Product 6}

\section{Crystal Growth}

Product $6(8 \mathrm{mg})$ was dissolved in a couple drops of $\mathrm{CH}_{2} \mathrm{Cl}_{2}$ combined with toluene $(\sim 0.5 \mathrm{~mL})$. The solution was transferred to an NMR tube and layered with pentane $(1.0 \mathrm{~mL})$. Single crystals suitable for X-ray diffraction grew at room temperature overnight.

\section{Experimental}

Low-temperature diffraction data ( $\omega$-scans) were collected on a Rigaku MicroMax-007HF diffractometer coupled to a Dectris Pilatus3R detector with Mo Ka $(\lambda=0.71073 \AA)$ for the structure of 007c-20063. The diffraction images were processed and scaled using Rigaku Oxford Diffraction software (CrysAlisPro; Rigaku OD: The Woodlands, TX, 2015). The structure was solved with SHELXT and was refined against $F^{2}$ on all data by full-matrix least squares with SHELXL (Sheldrick, G. M. Acta Cryst. 2008, A64, 112-122). All non-hydrogen atoms were refined anisotropically. Hydrogen atoms were included in the model at geometrically calculated positions and refined using a riding model. The isotropic displacement parameters of all hydrogen atoms were fixed to 1.2 times the $U$ value of the atoms to which they are linked (1.5 times for methyl groups). The full numbering scheme of compound 007c-20063 can be found in the full details of the X-ray structure determination (CIF), which is included as Supporting Information. CCDC number 2035056 (007c-20063) contains the supplementary crystallographic data for this paper. These data can be obtained free of charge from The Cambridge Crystallographic Data Center via www.ccdc.cam.ac.uk/data_request/cif. 


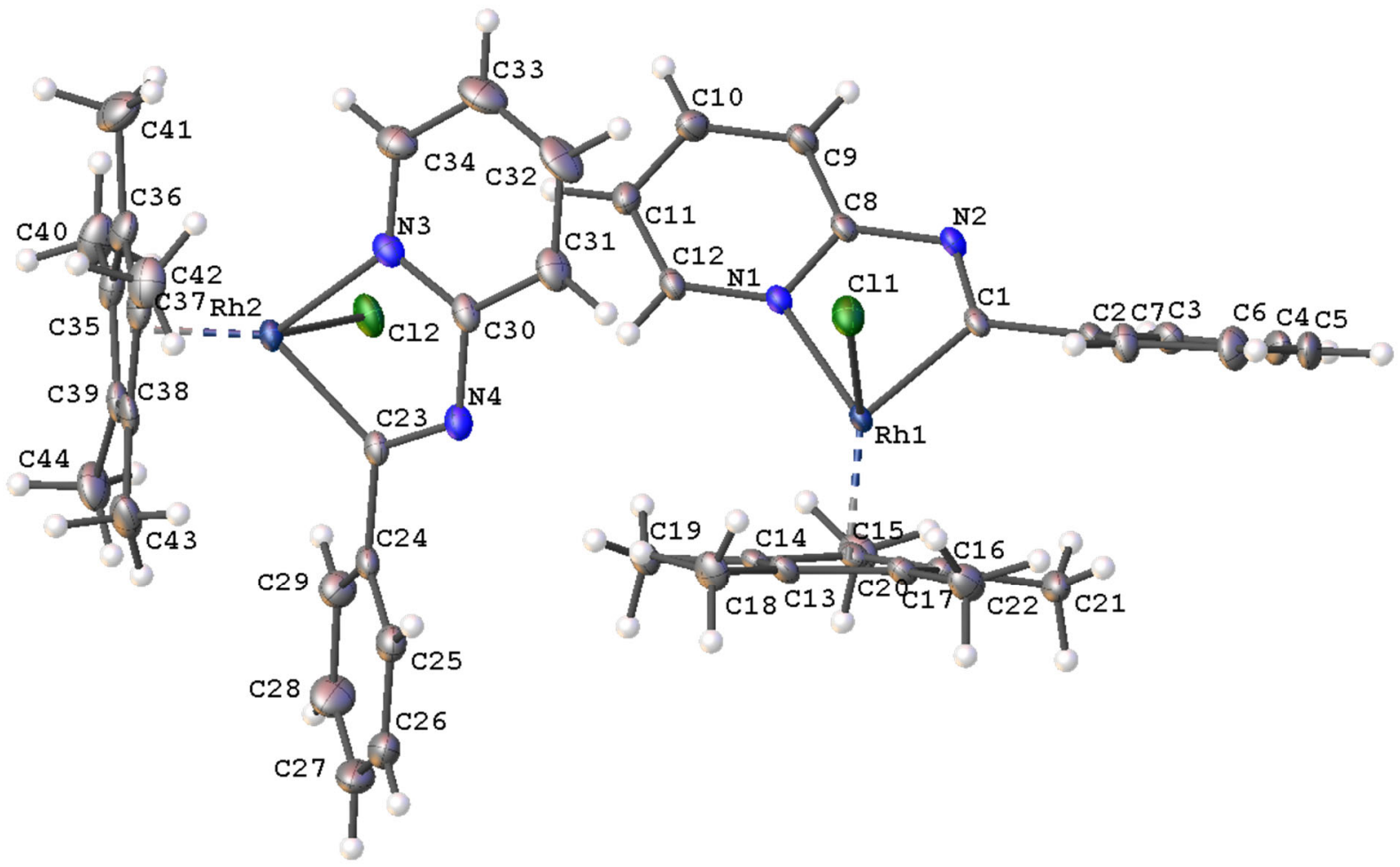

Figure 1. The complete numbering scheme of $007 \mathrm{c}-20063$ with $50 \%$ thermal ellipsoid probability levels. The hydrogen atoms are shown as circles for clarity. 
Table 1. Crystal data and structure refinement for 007c-20063.

Identification code

Empirical formula

Formula weight

Temperature

Wavelength

Crystal system

Space group

Unit cell dimensions

Volume

Z

Density (calculated)

Absorption coefficient

$\mathrm{F}(000)$

Crystal size

Crystal color and habit

Diffractometer

Theta range for data collection

Index ranges

Reflections collected

Independent reflections

Observed reflections $(\mathrm{I}>2$ sigma $(\mathrm{I}))$

Completeness to theta $=25.242^{\circ}$

Absorption correction

Max. and min. transmission

Solution method

Refinement method

Data / restraints / parameters

Goodness-of-fit on $\mathrm{F}^{2}$

Final $\mathrm{R}$ indices [l>2sigma(I)]

$\mathrm{R}$ indices (all data)

Largest diff. peak and hole 007c-20063

$\mathrm{C} 22 \mathrm{H} 24 \mathrm{Cl} \mathrm{N} 2 \mathrm{Rh}$

454.79

93(2) K

$0.71073 \AA$

Triclinic

P-1

$a=9.1949(2) \AA$ $\alpha=83.1610(10)^{\circ}$.

$\mathrm{b}=14.1206(2) \AA$ $\beta=77.4940(10)^{\circ}$.

$c=16.0991(2) \AA$ $\mathrm{Y}=78.843(2)^{\circ}$.

$1995.60(6) \AA^{3}$

4

$1.514 \mathrm{Mg} / \mathrm{m}^{3}$

$0.997 \mathrm{~mm}^{-1}$

928

$0.420 \times 0.300 \times 0.290 \mathrm{~mm}^{3}$

Red Block

Dectris Pilatus $3 R$

2.943 to $27.484^{\circ}$.

$-11<=\mathrm{h}<=11,-18<=\mathrm{k}<=18,-20<=\mathrm{l}<=20$

77139

$9121[R($ int $)=0.0443]$

8692

$99.8 \%$

Semi-empirical from equivalents

1.00000 and 0.61917

SHELXT-2014/5 (Sheldrick, 2014)

SHELXL-2014/7 (Sheldrick, 2014)

$9121 / 0 / 479$

\subsection{1}

$\mathrm{R} 1=0.0243, w R 2=0.0631$

$\mathrm{R} 1=0.0256, w R 2=0.0638$

1.093 and -0.921 e. $\AA^{-3}$ 
Table 2. Atomic coordinates $\left(x 10^{4}\right)$ and equivalent isotropic displacement parameters $\left(\AA^{2} \times 10^{3}\right)$ for $007 \mathrm{c}-20063$. $U(\mathrm{eq})$ is defined as one third of the trace of the orthogonalized Uij tensor.

\begin{tabular}{|c|c|c|c|c|}
\hline & $x$ & $y$ & $z$ & $\mathrm{U}(\mathrm{eq})$ \\
\hline $\mathrm{Rh}(1)$ & $1542(1)$ & $4712(1)$ & $2704(1)$ & $12(1)$ \\
\hline $\mathrm{Cl}(1)$ & $3964(1)$ & $3846(1)$ & $2062(1)$ & $22(1)$ \\
\hline $\mathrm{N}(1)$ & $1674(2)$ & $5742(1)$ & 1683(1) & $14(1)$ \\
\hline $\mathrm{N}(2)$ & $334(2)$ & $4748(1)$ & 1191(1) & $16(1)$ \\
\hline$C(1)$ & $548(2)$ & $4226(1)$ & 1886(1) & $14(1)$ \\
\hline$C(2)$ & $20(2)$ & $3277(1)$ & $2020(1)$ & $16(1)$ \\
\hline$C(3)$ & $-1454(2)$ & $3230(1)$ & $1954(1)$ & $20(1)$ \\
\hline$C(4)$ & $-1926(2)$ & $2335(2)$ & $2057(1)$ & $24(1)$ \\
\hline$C(5)$ & $-921(3)$ & 1494(2) & 2190(1) & $27(1)$ \\
\hline$C(6)$ & $546(2)$ & 1535(1) & $2257(1)$ & $27(1)$ \\
\hline$C(7)$ & $1006(2)$ & 2431(1) & $2189(1)$ & $21(1)$ \\
\hline$C(8)$ & $984(2)$ & $5576(1)$ & $1060(1)$ & $15(1)$ \\
\hline $\mathrm{C}(9)$ & $998(2)$ & $6185(1)$ & $312(1)$ & $20(1)$ \\
\hline$C(10)$ & 1772(2) & $6956(1)$ & $198(1)$ & $22(1)$ \\
\hline$C(11)$ & $2503(2)$ & $7113(1)$ & $826(1)$ & $20(1)$ \\
\hline$C(12)$ & 2423(2) & $6493(1)$ & 1558(1) & $17(1)$ \\
\hline$C(13)$ & 2192(2) & $4841(1)$ & $3968(1)$ & $16(1)$ \\
\hline$C(14)$ & $1170(2)$ & $5672(1)$ & $3791(1)$ & $15(1)$ \\
\hline$C(15)$ & $-208(2)$ & $5375(1)$ & $3679(1)$ & $16(1)$ \\
\hline$C(16)$ & $-46(2)$ & $4346(1)$ & $3869(1)$ & $17(1)$ \\
\hline$C(17)$ & $1457(2)$ & $3999(1)$ & $3986(1)$ & $17(1)$ \\
\hline$C(18)$ & $3778(2)$ & $4788(1)$ & $4082(1)$ & $23(1)$ \\
\hline$C(19)$ & 1383(2) & $6706(1)$ & $3722(1)$ & $20(1)$ \\
\hline$C(20)$ & $-1617(2)$ & $6042(1)$ & $3532(1)$ & $23(1)$ \\
\hline$C(21)$ & $-1280(2)$ & $3767(1)$ & $3966(1)$ & $22(1)$ \\
\hline$C(22)$ & $2149(2)$ & $2969(1)$ & $4184(1)$ & $25(1)$ \\
\hline $\mathrm{Rh}(2)$ & $5619(1)$ & $8899(1)$ & $2026(1)$ & $14(1)$ \\
\hline $\mathrm{Cl}(2)$ & $3154(1)$ & $9179(1)$ & $1702(1)$ & $22(1)$ \\
\hline$N(3)$ & $6132(2)$ & $7618(1)$ & $1433(1)$ & $18(1)$ \\
\hline $\mathrm{N}(4)$ & $5177(2)$ & $7009(1)$ & $2818(1)$ & $18(1)$ \\
\hline$C(23)$ & $4934(2)$ & $7920(1)$ & 2963(1) & $15(1)$ \\
\hline
\end{tabular}




\begin{tabular}{lrrrr}
$C(24)$ & $4125(2)$ & $8170(1)$ & $3833(1)$ & $18(1)$ \\
$C(25)$ & $4456(2)$ & $7586(1)$ & $4553(1)$ & $22(1)$ \\
$C(26)$ & $3687(2)$ & $7816(2)$ & $5365(1)$ & $27(1)$ \\
$C(27)$ & $2560(3)$ & $8618(2)$ & $5464(1)$ & $33(1)$ \\
$C(28)$ & $2212(3)$ & $9200(2)$ & $4754(2)$ & $34(1)$ \\
$C(29)$ & $2995(2)$ & $8985(1)$ & $3940(1)$ & $25(1)$ \\
$C(30)$ & $5823(2)$ & $6843(1)$ & $1974(1)$ & $18(1)$ \\
$C(31)$ & $6058(2)$ & $5918(1)$ & $1686(1)$ & $25(1)$ \\
$C(32)$ & $6591(3)$ & $5809(2)$ & $828(2)$ & $32(1)$ \\
$C(33)$ & $6888(3)$ & $6606(2)$ & $272(1)$ & $32(1)$ \\
$C(34)$ & $6649(2)$ & $7501(2)$ & $596(1)$ & $24(1)$ \\
$C(35)$ & $6031(2)$ & $10433(1)$ & $1569(1)$ & $22(1)$ \\
$C(36)$ & $7314(2)$ & $9788(1)$ & $1223(1)$ & $22(1)$ \\
$C(37)$ & $7861(2)$ & $9187(1)$ & $1927(1)$ & $20(1)$ \\
$C(38)$ & $6939(2)$ & $9524(1)$ & $2713(1)$ & $20(1)$ \\
$C(39)$ & $5749(2)$ & $10266(1)$ & $2495(1)$ & $20(1)$ \\
$C(40)$ & $5074(2)$ & $11176(2)$ & $1079(2)$ & $31(1)$ \\
$C(41)$ & $8027(3)$ & $9740(2)$ & $296(1)$ & $35(1)$ \\
$C(42)$ & $9277(2)$ & $8449(2)$ & $1841(2)$ & $30(1)$ \\
$C(43)$ & $7235(3)$ & $9187(2)$ & $3584(1)$ & $29(1)$ \\
$C(44)$ & $4600(2)$ & $10908(2)$ & $3081(2)$ & $31(1)$ \\
\hline & & & & \\
\hline
\end{tabular}


Table 3. Bond lengths $[\AA]$ and angles $\left[^{\circ}\right]$ for $007 c-20063$.

\begin{tabular}{|c|c|}
\hline $\mathrm{Rh}(1)-\mathrm{C}(1)$ & $2.0052(16)$ \\
\hline $\mathrm{Rh}(1)-\mathrm{N}(1)$ & $2.0600(14)$ \\
\hline $\mathrm{Rh}(1)-\mathrm{C}(15)$ & $2.1460(17)$ \\
\hline $\mathrm{Rh}(1)-\mathrm{C}(17)$ & $2.1788(17)$ \\
\hline $\mathrm{Rh}(1)-\mathrm{C}(16)$ & $2.1901(17)$ \\
\hline $\operatorname{Rh}(1)-C(14)$ & $2.2676(16)$ \\
\hline $\operatorname{Rh}(1)-C(13)$ & $2.2778(17)$ \\
\hline $\mathrm{Rh}(1)-\mathrm{Cl}(1)$ & $2.4021(4)$ \\
\hline $\mathrm{N}(1)-\mathrm{C}(12)$ & $1.346(2)$ \\
\hline$N(1)-C(8)$ & $1.360(2)$ \\
\hline$N(2)-C(1)$ & $1.294(2)$ \\
\hline$N(2)-C(8)$ & $1.387(2)$ \\
\hline$C(1)-C(2)$ & $1.488(2)$ \\
\hline$C(2)-C(7)$ & $1.391(3)$ \\
\hline$C(2)-C(3)$ & $1.397(2)$ \\
\hline$C(3)-C(4)$ & $1.394(3)$ \\
\hline $\mathrm{C}(3)-\mathrm{H}(3)$ & 0.9500 \\
\hline$C(4)-C(5)$ & $1.382(3)$ \\
\hline $\mathrm{C}(4)-\mathrm{H}(4)$ & 0.9500 \\
\hline$C(5)-C(6)$ & $1.389(3)$ \\
\hline $\mathrm{C}(5)-\mathrm{H}(5)$ & 0.9500 \\
\hline$C(6)-C(7)$ & $1.394(3)$ \\
\hline $\mathrm{C}(6)-\mathrm{H}(6)$ & 0.9500 \\
\hline $\mathrm{C}(7)-\mathrm{H}(7)$ & 0.9500 \\
\hline$C(8)-C(9)$ & $1.394(2)$ \\
\hline$C(9)-C(10)$ & $1.385(3)$ \\
\hline $\mathrm{C}(9)-\mathrm{H}(9)$ & 0.9500 \\
\hline$C(10)-C(11)$ & $1.389(3)$ \\
\hline $\mathrm{C}(10)-\mathrm{H}(10)$ & 0.9500 \\
\hline$C(11)-C(12)$ & $1.379(3)$ \\
\hline $\mathrm{C}(11)-\mathrm{H}(11)$ & 0.9500 \\
\hline $\mathrm{C}(12)-\mathrm{H}(12)$ & 0.9500 \\
\hline$C(13)-C(14)$ & $1.397(2)$ \\
\hline$C(13)-C(17)$ & $1.470(2)$ \\
\hline
\end{tabular}




\begin{tabular}{|c|c|}
\hline$C(13)-C(18)$ & $1.496(2)$ \\
\hline$C(14)-C(15)$ & $1.462(2)$ \\
\hline$C(14)-C(19)$ & $1.498(2)$ \\
\hline$C(15)-C(16)$ & $1.435(2)$ \\
\hline$C(15)-C(20)$ & $1.492(2)$ \\
\hline$C(16)-C(17)$ & $1.419(3)$ \\
\hline$C(16)-C(21)$ & $1.494(2)$ \\
\hline$C(17)-C(22)$ & $1.497(3)$ \\
\hline $\mathrm{C}(18)-\mathrm{H}(18 \mathrm{~A})$ & 0.9800 \\
\hline $\mathrm{C}(18)-\mathrm{H}(18 \mathrm{~B})$ & 0.9800 \\
\hline $\mathrm{C}(18)-\mathrm{H}(18 \mathrm{C})$ & 0.9800 \\
\hline $\mathrm{C}(19)-\mathrm{H}(19 \mathrm{~A})$ & 0.9800 \\
\hline $\mathrm{C}(19)-\mathrm{H}(19 \mathrm{~B})$ & 0.9800 \\
\hline $\mathrm{C}(19)-\mathrm{H}(19 \mathrm{C})$ & 0.9800 \\
\hline $\mathrm{C}(20)-\mathrm{H}(20 \mathrm{~A})$ & 0.9800 \\
\hline $\mathrm{C}(20)-\mathrm{H}(20 \mathrm{~B})$ & 0.9800 \\
\hline $\mathrm{C}(20)-\mathrm{H}(20 \mathrm{C})$ & 0.9800 \\
\hline $\mathrm{C}(21)-\mathrm{H}(21 \mathrm{~A})$ & 0.9800 \\
\hline $\mathrm{C}(21)-\mathrm{H}(21 \mathrm{~B})$ & 0.9800 \\
\hline $\mathrm{C}(21)-\mathrm{H}(21 \mathrm{C})$ & 0.9800 \\
\hline $\mathrm{C}(22)-\mathrm{H}(22 \mathrm{~A})$ & 0.9800 \\
\hline $\mathrm{C}(22)-\mathrm{H}(22 \mathrm{~B})$ & 0.9800 \\
\hline $\mathrm{C}(22)-\mathrm{H}(22 \mathrm{C})$ & 0.9800 \\
\hline $\mathrm{Rh}(2)-\mathrm{C}(23)$ & $2.0034(17)$ \\
\hline $\operatorname{Rh}(2)-N(3)$ & $2.0712(15)$ \\
\hline $\mathrm{Rh}(2)-\mathrm{C}(37)$ & $2.1470(17)$ \\
\hline $\mathrm{Rh}(2)-\mathrm{C}(38)$ & $2.1693(17)$ \\
\hline $\mathrm{Rh}(2)-\mathrm{C}(39)$ & $2.1870(17)$ \\
\hline $\operatorname{Rh}(2)-C(36)$ & $2.2742(18)$ \\
\hline $\mathrm{Rh}(2)-\mathrm{C}(35)$ & $2.2789(18)$ \\
\hline $\mathrm{Rh}(2)-\mathrm{Cl}(2)$ & $2.3832(4)$ \\
\hline$N(3)-C(34)$ & $1.348(2)$ \\
\hline$N(3)-C(30)$ & $1.351(2)$ \\
\hline$N(4)-C(23)$ & $1.303(2)$ \\
\hline$N(4)-C(30)$ & $1.388(2)$ \\
\hline$C(23)-C(24)$ & $1.484(2)$ \\
\hline
\end{tabular}




\begin{tabular}{|c|c|}
\hline$C(24)-C(29)$ & $1.394(3)$ \\
\hline$C(24)-C(25)$ & $1.394(3)$ \\
\hline$C(25)-C(26)$ & $1.388(3)$ \\
\hline $\mathrm{C}(25)-\mathrm{H}(25)$ & 0.9500 \\
\hline$C(26)-C(27)$ & $1.379(3)$ \\
\hline $\mathrm{C}(26)-\mathrm{H}(26)$ & 0.9500 \\
\hline $\mathrm{C}(27)-\mathrm{C}(28)$ & $1.384(3)$ \\
\hline $\mathrm{C}(27)-\mathrm{H}(27)$ & 0.9500 \\
\hline$C(28)-C(29)$ & $1.390(3)$ \\
\hline $\mathrm{C}(28)-\mathrm{H}(28)$ & 0.9500 \\
\hline $\mathrm{C}(29)-\mathrm{H}(29)$ & 0.9500 \\
\hline$C(30)-C(31)$ & $1.399(2)$ \\
\hline$C(31)-C(32)$ & $1.380(3)$ \\
\hline $\mathrm{C}(31)-\mathrm{H}(31)$ & 0.9500 \\
\hline $\mathrm{C}(32)-\mathrm{C}(33)$ & $1.384(3)$ \\
\hline $\mathrm{C}(32)-\mathrm{H}(32)$ & 0.9500 \\
\hline$C(33)-C(34)$ & $1.385(3)$ \\
\hline $\mathrm{C}(33)-\mathrm{H}(33)$ & 0.9500 \\
\hline $\mathrm{C}(34)-\mathrm{H}(34)$ & 0.9500 \\
\hline$C(35)-C(36)$ & $1.397(3)$ \\
\hline$C(35)-C(39)$ & $1.455(3)$ \\
\hline$C(35)-C(40)$ & $1.498(3)$ \\
\hline $\mathrm{C}(36)-\mathrm{C}(37)$ & $1.452(3)$ \\
\hline$C(36)-C(41)$ & $1.499(3)$ \\
\hline $\mathrm{C}(37)-\mathrm{C}(38)$ & $1.439(3)$ \\
\hline$C(37)-C(42)$ & $1.494(3)$ \\
\hline $\mathrm{C}(38)-\mathrm{C}(39)$ & $1.433(3)$ \\
\hline$C(38)-C(43)$ & $1.491(3)$ \\
\hline$C(39)-C(44)$ & $1.494(3)$ \\
\hline $\mathrm{C}(40)-\mathrm{H}(40 \mathrm{~A})$ & 0.9800 \\
\hline $\mathrm{C}(40)-\mathrm{H}(40 \mathrm{~B})$ & 0.9800 \\
\hline $\mathrm{C}(40)-\mathrm{H}(40 \mathrm{C})$ & 0.9800 \\
\hline $\mathrm{C}(41)-\mathrm{H}(41 \mathrm{~A})$ & 0.9800 \\
\hline $\mathrm{C}(41)-\mathrm{H}(41 \mathrm{~B})$ & 0.9800 \\
\hline $\mathrm{C}(41)-\mathrm{H}(41 \mathrm{C})$ & 0.9800 \\
\hline $\mathrm{C}(42)-\mathrm{H}(42 \mathrm{~A})$ & 0.9800 \\
\hline
\end{tabular}




\begin{tabular}{|c|c|}
\hline $\mathrm{C}(42)-\mathrm{H}(42 \mathrm{~B})$ & 0.9800 \\
\hline $\mathrm{C}(42)-\mathrm{H}(42 \mathrm{C})$ & 0.9800 \\
\hline $\mathrm{C}(43)-\mathrm{H}(43 \mathrm{~A})$ & 0.9800 \\
\hline $\mathrm{C}(43)-\mathrm{H}(43 \mathrm{~B})$ & 0.9800 \\
\hline $\mathrm{C}(43)-\mathrm{H}(43 \mathrm{C})$ & 0.9800 \\
\hline $\mathrm{C}(44)-\mathrm{H}(44 \mathrm{~A})$ & 0.9800 \\
\hline $\mathrm{C}(44)-\mathrm{H}(44 \mathrm{~B})$ & 0.9800 \\
\hline $\mathrm{C}(44)-\mathrm{H}(44 \mathrm{C})$ & 0.9800 \\
\hline $\mathrm{C}(1)-\mathrm{Rh}(1)-\mathrm{N}(1)$ & $76.30(6)$ \\
\hline$C(1)-R h(1)-C(15)$ & $107.82(7)$ \\
\hline$N(1)-R h(1)-C(15)$ & $104.99(6)$ \\
\hline $\mathrm{C}(1)-\mathrm{Rh}(1)-\mathrm{C}(17)$ & $119.69(7)$ \\
\hline $\mathrm{N}(1)-\mathrm{Rh}(1)-\mathrm{C}(17)$ & $162.30(6)$ \\
\hline $\mathrm{C}(15)-\mathrm{Rh}(1)-\mathrm{C}(17)$ & $64.41(7)$ \\
\hline$C(1)-R h(1)-C(16)$ & $96.82(7)$ \\
\hline$N(1)-R h(1)-C(16)$ & $139.73(6)$ \\
\hline$C(15)-\operatorname{Rh}(1)-C(16)$ & $38.62(6)$ \\
\hline$C(17)-\operatorname{Rh}(1)-C(16)$ & $37.92(7)$ \\
\hline$C(1)-\operatorname{Rh}(1)-C(14)$ & $144.91(7)$ \\
\hline $\mathrm{N}(1)-\mathrm{Rh}(1)-\mathrm{C}(14)$ & $99.94(6)$ \\
\hline$C(15)-R h(1)-C(14)$ & $38.56(6)$ \\
\hline $\mathrm{C}(17)-\mathrm{Rh}(1)-\mathrm{C}(14)$ & $62.76(6)$ \\
\hline$C(16)-\operatorname{Rh}(1)-C(14)$ & $63.23(6)$ \\
\hline $\mathrm{C}(1)-\mathrm{Rh}(1)-\mathrm{C}(13)$ & $157.83(7)$ \\
\hline $\mathrm{N}(1)-\mathrm{Rh}(1)-\mathrm{C}(13)$ & $124.82(6)$ \\
\hline$C(15)-\operatorname{Rh}(1)-C(13)$ & $63.12(6)$ \\
\hline$C(17)-\operatorname{Rh}(1)-C(13)$ & $38.45(6)$ \\
\hline$C(16)-\operatorname{Rh}(1)-C(13)$ & $63.10(6)$ \\
\hline$C(14)-\operatorname{Rh}(1)-C(13)$ & $35.80(6)$ \\
\hline$C(1)-\operatorname{Rh}(1)-C l(1)$ & $90.85(5)$ \\
\hline $\mathrm{N}(1)-\mathrm{Rh}(1)-\mathrm{Cl}(1)$ & $89.67(4)$ \\
\hline $\mathrm{C}(15)-\mathrm{Rh}(1)-\mathrm{Cl}(1)$ & $158.38(5)$ \\
\hline $\mathrm{C}(17)-\mathrm{Rh}(1)-\mathrm{Cl}(1)$ & $97.16(5)$ \\
\hline $\mathrm{C}(16)-\mathrm{Rh}(1)-\mathrm{Cl}(1)$ & $130.44(5)$ \\
\hline $\mathrm{C}(14)-\mathrm{Rh}(1)-\mathrm{Cl}(1)$ & $124.20(5)$ \\
\hline
\end{tabular}




\begin{tabular}{|c|c|}
\hline $\mathrm{C}(13)-\mathrm{Rh}(1)-\mathrm{Cl}(1)$ & $95.52(5)$ \\
\hline $\mathrm{C}(12)-\mathrm{N}(1)-\mathrm{C}(8)$ & $119.05(15)$ \\
\hline $\mathrm{C}(12)-\mathrm{N}(1)-\mathrm{Rh}(1)$ & $127.34(12)$ \\
\hline $\mathrm{C}(8)-\mathrm{N}(1)-\mathrm{Rh}(1)$ & $113.49(11)$ \\
\hline $\mathrm{C}(1)-\mathrm{N}(2)-\mathrm{C}(8)$ & $113.37(14)$ \\
\hline$N(2)-C(1)-C(2)$ & $116.12(15)$ \\
\hline$N(2)-C(1)-R h(1)$ & $119.88(12)$ \\
\hline$C(2)-C(1)-R h(1)$ & $124.00(12)$ \\
\hline$C(7)-C(2)-C(3)$ & $119.74(16)$ \\
\hline$C(7)-C(2)-C(1)$ & $120.06(16)$ \\
\hline$C(3)-C(2)-C(1)$ & $120.18(16)$ \\
\hline$C(4)-C(3)-C(2)$ & $119.81(18)$ \\
\hline $\mathrm{C}(4)-\mathrm{C}(3)-\mathrm{H}(3)$ & 120.1 \\
\hline $\mathrm{C}(2)-\mathrm{C}(3)-\mathrm{H}(3)$ & 120.1 \\
\hline$C(5)-C(4)-C(3)$ & $120.09(18)$ \\
\hline $\mathrm{C}(5)-\mathrm{C}(4)-\mathrm{H}(4)$ & 120.0 \\
\hline $\mathrm{C}(3)-\mathrm{C}(4)-\mathrm{H}(4)$ & 120.0 \\
\hline$C(4)-C(5)-C(6)$ & $120.40(18)$ \\
\hline $\mathrm{C}(4)-\mathrm{C}(5)-\mathrm{H}(5)$ & 119.8 \\
\hline $\mathrm{C}(6)-\mathrm{C}(5)-\mathrm{H}(5)$ & 119.8 \\
\hline$C(5)-C(6)-C(7)$ & $119.69(19)$ \\
\hline $\mathrm{C}(5)-\mathrm{C}(6)-\mathrm{H}(6)$ & 120.2 \\
\hline $\mathrm{C}(7)-\mathrm{C}(6)-\mathrm{H}(6)$ & 120.2 \\
\hline$C(2)-C(7)-C(6)$ & $120.16(18)$ \\
\hline $\mathrm{C}(2)-\mathrm{C}(7)-\mathrm{H}(7)$ & 119.9 \\
\hline $\mathrm{C}(6)-\mathrm{C}(7)-\mathrm{H}(7)$ & 119.9 \\
\hline$N(1)-C(8)-N(2)$ & $116.65(15)$ \\
\hline$N(1)-C(8)-C(9)$ & $121.33(15)$ \\
\hline$N(2)-C(8)-C(9)$ & $121.94(15)$ \\
\hline$C(10)-C(9)-C(8)$ & $118.64(17)$ \\
\hline $\mathrm{C}(10)-\mathrm{C}(9)-\mathrm{H}(9)$ & 120.7 \\
\hline $\mathrm{C}(8)-\mathrm{C}(9)-\mathrm{H}(9)$ & 120.7 \\
\hline$C(9)-C(10)-C(11)$ & $119.97(17)$ \\
\hline$C(9)-C(10)-H(10)$ & 120.0 \\
\hline $\mathrm{C}(11)-\mathrm{C}(10)-\mathrm{H}(10)$ & 120.0 \\
\hline$C(12)-C(11)-C(10)$ & $118.48(16)$ \\
\hline
\end{tabular}




\begin{tabular}{|c|c|}
\hline$C(12)-C(11)-H(11)$ & 120.8 \\
\hline $\mathrm{C}(10)-\mathrm{C}(11)-\mathrm{H}(11)$ & 120.8 \\
\hline$N(1)-C(12)-C(11)$ & $122.50(16)$ \\
\hline $\mathrm{N}(1)-\mathrm{C}(12)-\mathrm{H}(12)$ & 118.8 \\
\hline $\mathrm{C}(11)-\mathrm{C}(12)-\mathrm{H}(12)$ & 118.8 \\
\hline$C(14)-C(13)-C(17)$ & $107.75(15)$ \\
\hline$C(14)-C(13)-C(18)$ & $127.23(16)$ \\
\hline$C(17)-C(13)-C(18)$ & $124.97(16)$ \\
\hline$C(14)-C(13)-R h(1)$ & $71.70(10)$ \\
\hline$C(17)-C(13)-R h(1)$ & $67.13(9)$ \\
\hline$C(18)-C(13)-R h(1)$ & $124.46(13)$ \\
\hline$C(13)-C(14)-C(15)$ & $108.32(15)$ \\
\hline$C(13)-C(14)-C(19)$ & $127.93(16)$ \\
\hline$C(15)-C(14)-C(19)$ & $123.74(16)$ \\
\hline$C(13)-C(14)-R h(1)$ & $72.50(10)$ \\
\hline$C(15)-C(14)-R h(1)$ & $66.22(9)$ \\
\hline$C(19)-C(14)-R h(1)$ & $126.91(12)$ \\
\hline$C(16)-C(15)-C(14)$ & $107.63(15)$ \\
\hline$C(16)-C(15)-C(20)$ & $126.08(16)$ \\
\hline$C(14)-C(15)-C(20)$ & $125.62(16)$ \\
\hline$C(16)-C(15)-R h(1)$ & $72.35(10)$ \\
\hline$C(14)-C(15)-\operatorname{Rh}(1)$ & $75.22(10)$ \\
\hline$C(20)-C(15)-R h(1)$ & $125.49(12)$ \\
\hline$C(17)-C(16)-C(15)$ & $107.73(15)$ \\
\hline$C(17)-C(16)-C(21)$ & $127.05(17)$ \\
\hline$C(15)-C(16)-C(21)$ & $125.16(17)$ \\
\hline$C(17)-C(16)-R h(1)$ & $70.61(10)$ \\
\hline$C(15)-C(16)-R h(1)$ & $69.03(9)$ \\
\hline$C(21)-C(16)-R h(1)$ & $127.98(12)$ \\
\hline$C(16)-C(17)-C(13)$ & $108.06(15)$ \\
\hline$C(16)-C(17)-C(22)$ & $126.77(16)$ \\
\hline$C(13)-C(17)-C(22)$ & $124.89(16)$ \\
\hline$C(16)-C(17)-R h(1)$ & $71.47(10)$ \\
\hline$C(13)-C(17)-R h(1)$ & $74.42(10)$ \\
\hline$C(22)-C(17)-R h(1)$ & $124.65(13)$ \\
\hline$C(13)-C(18)-H(18 A)$ & 109.5 \\
\hline
\end{tabular}




\begin{tabular}{|c|c|}
\hline$C(13)-C(18)-H(18 B)$ & 109.5 \\
\hline$H(18 A)-C(18)-H(18 B)$ & 109.5 \\
\hline $\mathrm{C}(13)-\mathrm{C}(18)-\mathrm{H}(18 \mathrm{C})$ & 109.5 \\
\hline$H(18 A)-C(18)-H(18 C)$ & 109.5 \\
\hline$H(18 B)-C(18)-H(18 C)$ & 109.5 \\
\hline$C(14)-C(19)-H(19 A)$ & 109.5 \\
\hline$C(14)-C(19)-H(19 B)$ & 109.5 \\
\hline$H(19 A)-C(19)-H(19 B)$ & 109.5 \\
\hline$C(14)-C(19)-H(19 C)$ & 109.5 \\
\hline$H(19 A)-C(19)-H(19 C)$ & 109.5 \\
\hline$H(19 B)-C(19)-H(19 C)$ & 109.5 \\
\hline$C(15)-C(20)-H(20 A)$ & 109.5 \\
\hline$C(15)-C(20)-H(20 B)$ & 109.5 \\
\hline$H(20 A)-C(20)-H(20 B)$ & 109.5 \\
\hline$C(15)-C(20)-H(20 C)$ & 109.5 \\
\hline$H(20 A)-C(20)-H(20 C)$ & 109.5 \\
\hline$H(20 B)-C(20)-H(20 C)$ & 109.5 \\
\hline$C(16)-C(21)-H(21 A)$ & 109.5 \\
\hline$C(16)-C(21)-H(21 B)$ & 109.5 \\
\hline$H(21 A)-C(21)-H(21 B)$ & 109.5 \\
\hline$C(16)-C(21)-H(21 C)$ & 109.5 \\
\hline$H(21 A)-C(21)-H(21 C)$ & 109.5 \\
\hline$H(21 B)-C(21)-H(21 C)$ & 109.5 \\
\hline $\mathrm{C}(17)-\mathrm{C}(22)-\mathrm{H}(22 \mathrm{~A})$ & 109.5 \\
\hline $\mathrm{C}(17)-\mathrm{C}(22)-\mathrm{H}(22 \mathrm{~B})$ & 109.5 \\
\hline $\mathrm{H}(22 \mathrm{~A})-\mathrm{C}(22)-\mathrm{H}(22 \mathrm{~B})$ & 109.5 \\
\hline $\mathrm{C}(17)-\mathrm{C}(22)-\mathrm{H}(22 \mathrm{C})$ & 109.5 \\
\hline$H(22 A)-C(22)-H(22 C)$ & 109.5 \\
\hline $\mathrm{H}(22 \mathrm{~B})-\mathrm{C}(22)-\mathrm{H}(22 \mathrm{C})$ & 109.5 \\
\hline $\mathrm{C}(23)-\mathrm{Rh}(2)-\mathrm{N}(3)$ & $76.43(7)$ \\
\hline$C(23)-\operatorname{Rh}(2)-C(37)$ & $116.32(7)$ \\
\hline $\mathrm{N}(3)-\mathrm{Rh}(2)-\mathrm{C}(37)$ & $99.70(7)$ \\
\hline$C(23)-\operatorname{Rh}(2)-C(38)$ & $96.92(7)$ \\
\hline $\mathrm{N}(3)-\mathrm{Rh}(2)-\mathrm{C}(38)$ & $130.17(7)$ \\
\hline $\mathrm{C}(37)-\mathrm{Rh}(2)-\mathrm{C}(38)$ & $38.93(7)$ \\
\hline $\mathrm{C}(23)-\mathrm{Rh}(2)-\mathrm{C}(39)$ & $112.98(7)$ \\
\hline
\end{tabular}




\begin{tabular}{|c|c|}
\hline $\mathrm{N}(3)-\mathrm{Rh}(2)-\mathrm{C}(39)$ & $163.75(7)$ \\
\hline $\mathrm{C}(37)-\mathrm{Rh}(2)-\mathrm{C}(39)$ & $64.47(7)$ \\
\hline $\mathrm{C}(38)-\operatorname{Rh}(2)-\mathrm{C}(39)$ & $38.40(7)$ \\
\hline $\mathrm{C}(23)-\mathrm{Rh}(2)-\mathrm{C}(36)$ & $154.50(7)$ \\
\hline $\mathrm{N}(3)-\operatorname{Rh}(2)-\mathrm{C}(36)$ & $102.88(7)$ \\
\hline $\mathrm{C}(37)-\mathrm{Rh}(2)-\mathrm{C}(36)$ & $38.22(7)$ \\
\hline $\mathrm{C}(38)-\operatorname{Rh}(2)-\mathrm{C}(36)$ & $63.61(7)$ \\
\hline $\mathrm{C}(39)-\operatorname{Rh}(2)-\mathrm{C}(36)$ & $62.72(7)$ \\
\hline $\mathrm{C}(23)-\operatorname{Rh}(2)-\mathrm{C}(35)$ & $150.49(7)$ \\
\hline N(3)-Rh(2)-C(35) & $132.90(7)$ \\
\hline $\mathrm{C}(37)-\mathrm{Rh}(2)-\mathrm{C}(35)$ & $62.54(7)$ \\
\hline $\mathrm{C}(38)-\operatorname{Rh}(2)-\mathrm{C}(35)$ & $63.10(7)$ \\
\hline $\mathrm{C}(39)-\operatorname{Rh}(2)-\mathrm{C}(35)$ & $37.97(7)$ \\
\hline $\mathrm{C}(36)-\operatorname{Rh}(2)-\mathrm{C}(35)$ & $35.74(7)$ \\
\hline $\mathrm{C}(23)-\mathrm{Rh}(2)-\mathrm{Cl}(2)$ & $88.33(5)$ \\
\hline $\mathrm{N}(3)-\mathrm{Rh}(2)-\mathrm{Cl}(2)$ & $89.70(4)$ \\
\hline $\mathrm{C}(37)-\mathrm{Rh}(2)-\mathrm{Cl}(2)$ & $155.00(5)$ \\
\hline $\mathrm{C}(38)-\mathrm{Rh}(2)-\mathrm{Cl}(2)$ & $139.96(5)$ \\
\hline $\mathrm{C}(39)-\mathrm{Rh}(2)-\mathrm{Cl}(2)$ & $103.42(5)$ \\
\hline $\mathrm{C}(36)-\mathrm{Rh}(2)-\mathrm{Cl}(2)$ & $117.16(5)$ \\
\hline $\mathrm{C}(35)-\mathrm{Rh}(2)-\mathrm{Cl}(2)$ & $94.15(5)$ \\
\hline $\mathrm{C}(34)-\mathrm{N}(3)-\mathrm{C}(30)$ & $119.19(16)$ \\
\hline $\mathrm{C}(34)-\mathrm{N}(3)-\mathrm{Rh}(2)$ & $127.58(13)$ \\
\hline $\mathrm{C}(30)-\mathrm{N}(3)-\mathrm{Rh}(2)$ & $113.15(12)$ \\
\hline $\mathrm{C}(23)-\mathrm{N}(4)-\mathrm{C}(30)$ & $113.23(15)$ \\
\hline$N(4)-C(23)-C(24)$ & $116.60(15)$ \\
\hline $\mathrm{N}(4)-\mathrm{C}(23)-\mathrm{Rh}(2)$ & $119.71(13)$ \\
\hline$C(24)-C(23)-\operatorname{Rh}(2)$ & $123.68(12)$ \\
\hline$C(29)-C(24)-C(25)$ & $118.93(17)$ \\
\hline$C(29)-C(24)-C(23)$ & $120.18(17)$ \\
\hline$C(25)-C(24)-C(23)$ & $120.88(16)$ \\
\hline$C(26)-C(25)-C(24)$ & $120.76(19)$ \\
\hline $\mathrm{C}(26)-\mathrm{C}(25)-\mathrm{H}(25)$ & 119.6 \\
\hline $\mathrm{C}(24)-\mathrm{C}(25)-\mathrm{H}(25)$ & 119.6 \\
\hline$C(27)-C(26)-C(25)$ & $119.9(2)$ \\
\hline $\mathrm{C}(27)-\mathrm{C}(26)-\mathrm{H}(26)$ & 120.1 \\
\hline
\end{tabular}




\begin{tabular}{ll}
$\mathrm{C}(25)-\mathrm{C}(26)-\mathrm{H}(26)$ & 120.1 \\
$\mathrm{C}(26)-\mathrm{C}(27)-\mathrm{C}(28)$ & $119.9(2)$ \\
$\mathrm{C}(26)-\mathrm{C}(27)-\mathrm{H}(27)$ & 120.0 \\
$\mathrm{C}(28)-\mathrm{C}(27)-\mathrm{H}(27)$ & 120.0 \\
$\mathrm{C}(27)-\mathrm{C}(28)-\mathrm{C}(29)$ & $120.6(2)$ \\
$\mathrm{C}(27)-\mathrm{C}(28)-\mathrm{H}(28)$ & 119.7 \\
$\mathrm{C}(29)-\mathrm{C}(28)-\mathrm{H}(28)$ & 119.7 \\
$\mathrm{C}(28)-\mathrm{C}(29)-\mathrm{C}(24)$ & $119.9(2)$ \\
$\mathrm{C}(28)-\mathrm{C}(29)-\mathrm{H}(29)$ & 120.0 \\
$\mathrm{C}(24)-\mathrm{C}(29)-\mathrm{H}(29)$ & 120.0 \\
$\mathrm{~N}(3)-\mathrm{C}(30)-\mathrm{N}(4)$ & $117.28(15)$ \\
$\mathrm{N}(3)-\mathrm{C}(30)-\mathrm{C}(31)$ & $121.42(17)$ \\
$\mathrm{N}(4)-\mathrm{C}(30)-\mathrm{C}(31)$ & $121.19(17)$ \\
$\mathrm{C}(32)-\mathrm{C}(31)-\mathrm{C}(30)$ & $118.69(19)$ \\
$\mathrm{C}(32)-\mathrm{C}(31)-\mathrm{H}(31)$ & 120.7 \\
$\mathrm{C}(30)-\mathrm{C}(31)-\mathrm{H}(31)$ & 120.7 \\
$\mathrm{C}(31)-\mathrm{C}(32)-\mathrm{C}(33)$ & $119.89(19)$ \\
$\mathrm{C}(31)-\mathrm{C}(32)-\mathrm{H}(32)$ & 120.1 \\
$\mathrm{C}(33)-\mathrm{C}(32)-\mathrm{H}(32)$ & 120.1 \\
$\mathrm{C}(32)-\mathrm{C}(33)-\mathrm{C}(34)$ & $118.8(2)$ \\
$\mathrm{C}(32)-\mathrm{C}(33)-\mathrm{H}(33)$ & 120.6 \\
$\mathrm{C}(34)-\mathrm{C}(33)-\mathrm{H}(33)$ & 120.6 \\
$\mathrm{~N}(3)-\mathrm{C}(34)-\mathrm{C}(33)$ & $122.0(2)$ \\
$\mathrm{N}(3)-\mathrm{C}(34)-\mathrm{H}(34)$ & 119.0 \\
$\mathrm{C}(33)-\mathrm{C}(34)-\mathrm{H}(34)$ & 119.0 \\
$\mathrm{C}(36)-\mathrm{C}(35)-\mathrm{C}(39)$ & $109.03(16)$ \\
$\mathrm{C}(36)-\mathrm{C}(35)-\mathrm{C}(40)$ & $126.34(19)$ \\
$\mathrm{C}(39)-\mathrm{C}(35)-\mathrm{C}(40)$ & $124.63(19)$ \\
$\mathrm{C}(36)-\mathrm{C}(35)-\mathrm{Rh}(2)$ & $71.95(10)$ \\
$\mathrm{C}(39)-\mathrm{C}(35)-\mathrm{Rh}(2)$ & $67.59(10)$ \\
$\mathrm{C}(40)-\mathrm{C}(35)-\mathrm{Rh}(2)$ & $126.03(13)$ \\
$\mathrm{C}(35)-\mathrm{C}(36)-\mathrm{C}(37)$ & $107.64(17)$ \\
$\mathrm{C}(35)-\mathrm{C}(36)-\mathrm{C}(41)$ & $126.36(19)$ \\
& \\
$\mathrm{C}(36)-\mathrm{Rh}(2)$ & $125.98(19)$ \\
& \\
$\mathrm{C}(31)$ & $14(10)$ \\
\hline
\end{tabular}




\begin{tabular}{|c|c|}
\hline$C(41)-C(36)-\operatorname{Rh}(2)$ & $128.15(14)$ \\
\hline$C(38)-C(37)-C(36)$ & $108.30(16)$ \\
\hline$C(38)-C(37)-C(42)$ & $126.10(18)$ \\
\hline$C(36)-C(37)-C(42)$ & $125.02(19)$ \\
\hline $\mathrm{C}(38)-\mathrm{C}(37)-\mathrm{Rh}(2)$ & $71.37(10)$ \\
\hline $\mathrm{C}(36)-\mathrm{C}(37)-\mathrm{Rh}(2)$ & $75.64(10)$ \\
\hline$C(42)-C(37)-\operatorname{Rh}(2)$ & $125.77(13)$ \\
\hline $\mathrm{C}(39)-\mathrm{C}(38)-\mathrm{C}(37)$ & $107.25(16)$ \\
\hline$C(39)-C(38)-C(43)$ & $127.16(19)$ \\
\hline$C(37)-C(38)-C(43)$ & $125.53(18)$ \\
\hline $\mathrm{C}(39)-\mathrm{C}(38)-\mathrm{Rh}(2)$ & $71.47(10)$ \\
\hline $\mathrm{C}(37)-\mathrm{C}(38)-\mathrm{Rh}(2)$ & $69.70(10)$ \\
\hline $\mathrm{C}(43)-\mathrm{C}(38)-\mathrm{Rh}(2)$ & $126.34(13)$ \\
\hline $\mathrm{C}(38)-\mathrm{C}(39)-\mathrm{C}(35)$ & $107.51(17)$ \\
\hline $\mathrm{C}(38)-\mathrm{C}(39)-\mathrm{C}(44)$ & $127.57(19)$ \\
\hline $\mathrm{C}(35)-\mathrm{C}(39)-\mathrm{C}(44)$ & $123.82(18)$ \\
\hline C(38)-C(39)-Rh(2) & $70.13(10)$ \\
\hline $\mathrm{C}(35)-\mathrm{C}(39)-\mathrm{Rh}(2)$ & $74.44(10)$ \\
\hline $\mathrm{C}(44)-\mathrm{C}(39)-\mathrm{Rh}(2)$ & $130.29(13)$ \\
\hline $\mathrm{C}(35)-\mathrm{C}(40)-\mathrm{H}(40 \mathrm{~A})$ & 109.5 \\
\hline $\mathrm{C}(35)-\mathrm{C}(40)-\mathrm{H}(40 \mathrm{~B})$ & 109.5 \\
\hline $\mathrm{H}(40 \mathrm{~A})-\mathrm{C}(40)-\mathrm{H}(40 \mathrm{~B})$ & 109.5 \\
\hline $\mathrm{C}(35)-\mathrm{C}(40)-\mathrm{H}(40 \mathrm{C})$ & 109.5 \\
\hline $\mathrm{H}(40 \mathrm{~A})-\mathrm{C}(40)-\mathrm{H}(40 \mathrm{C})$ & 109.5 \\
\hline $\mathrm{H}(40 \mathrm{~B})-\mathrm{C}(40)-\mathrm{H}(40 \mathrm{C})$ & 109.5 \\
\hline $\mathrm{C}(36)-\mathrm{C}(41)-\mathrm{H}(41 \mathrm{~A})$ & 109.5 \\
\hline $\mathrm{C}(36)-\mathrm{C}(41)-\mathrm{H}(41 \mathrm{~B})$ & 109.5 \\
\hline $\mathrm{H}(41 \mathrm{~A})-\mathrm{C}(41)-\mathrm{H}(41 \mathrm{~B})$ & 109.5 \\
\hline $\mathrm{C}(36)-\mathrm{C}(41)-\mathrm{H}(41 \mathrm{C})$ & 109.5 \\
\hline $\mathrm{H}(41 \mathrm{~A})-\mathrm{C}(41)-\mathrm{H}(41 \mathrm{C})$ & 109.5 \\
\hline $\mathrm{H}(41 \mathrm{~B})-\mathrm{C}(41)-\mathrm{H}(41 \mathrm{C})$ & 109.5 \\
\hline $\mathrm{C}(37)-\mathrm{C}(42)-\mathrm{H}(42 \mathrm{~A})$ & 109.5 \\
\hline $\mathrm{C}(37)-\mathrm{C}(42)-\mathrm{H}(42 \mathrm{~B})$ & 109.5 \\
\hline $\mathrm{H}(42 \mathrm{~A})-\mathrm{C}(42)-\mathrm{H}(42 \mathrm{~B})$ & 109.5 \\
\hline $\mathrm{C}(37)-\mathrm{C}(42)-\mathrm{H}(42 \mathrm{C})$ & 109.5 \\
\hline $\mathrm{H}(42 \mathrm{~A})-\mathrm{C}(42)-\mathrm{H}(42 \mathrm{C})$ & 109.5 \\
\hline
\end{tabular}




\begin{tabular}{ll}
$\mathrm{H}(42 \mathrm{~B})-\mathrm{C}(42)-\mathrm{H}(42 \mathrm{C})$ & 109.5 \\
$\mathrm{C}(38)-\mathrm{C}(43)-\mathrm{H}(43 A)$ & 109.5 \\
$\mathrm{C}(38)-\mathrm{C}(43)-\mathrm{H}(43 B)$ & 109.5 \\
$\mathrm{H}(43 \mathrm{~A})-\mathrm{C}(43)-\mathrm{H}(43 \mathrm{~B})$ & 109.5 \\
$\mathrm{C}(38)-\mathrm{C}(43)-\mathrm{H}(43 \mathrm{C})$ & 109.5 \\
$\mathrm{H}(43 \mathrm{~A})-\mathrm{C}(43)-\mathrm{H}(43 \mathrm{C})$ & 109.5 \\
$\mathrm{H}(43 \mathrm{~B})-\mathrm{C}(43)-\mathrm{H}(43 \mathrm{C})$ & 109.5 \\
$\mathrm{C}(39)-\mathrm{C}(44)-\mathrm{H}(44 \mathrm{~A})$ & 109.5 \\
$\mathrm{C}(39)-\mathrm{C}(44)-\mathrm{H}(44 \mathrm{~B})$ & 109.5 \\
$\mathrm{H}(44 \mathrm{~A})-\mathrm{C}(44)-\mathrm{H}(44 \mathrm{~B})$ & 109.5 \\
$\mathrm{C}(39)-\mathrm{C}(44)-\mathrm{H}(44 \mathrm{C})$ & 109.5 \\
$\mathrm{H}(44 \mathrm{~A})-\mathrm{C}(44)-\mathrm{H}(44 \mathrm{C})$ & 109.5 \\
$\mathrm{H}(44 \mathrm{~B})-\mathrm{C}(44)-\mathrm{H}(44 \mathrm{C})$ & 109.5 \\
\hline
\end{tabular}

Symmetry transformations used to generate equivalent atoms: 
Table 4. Anisotropic displacement parameters $\left(\AA^{2} \times 10^{3}\right)$ for 007c-20063. The anisotropic displacement factor exponent takes the form: $-2 \pi^{2}\left[h^{2} a^{* 2} U^{11}+\ldots+2 h k a^{*} b^{*} U^{12}\right]$

\begin{tabular}{|c|c|c|c|c|c|c|}
\hline & $u^{11}$ & $u^{22}$ & $u^{33}$ & $u^{23}$ & $u^{13}$ & $u^{12}$ \\
\hline $\mathrm{Rh}(1)$ & $13(1)$ & 11(1) & $13(1)$ & $-4(1)$ & $-3(1)$ & $-3(1)$ \\
\hline $\mathrm{Cl}(1)$ & $17(1)$ & $18(1)$ & $30(1)$ & $-8(1)$ & $-1(1)$ & $-2(1)$ \\
\hline $\mathrm{N}(1)$ & $14(1)$ & $13(1)$ & $14(1)$ & $-4(1)$ & $-2(1)$ & $-3(1)$ \\
\hline $\mathrm{N}(2)$ & $20(1)$ & $15(1)$ & $16(1)$ & $-6(1)$ & $-4(1)$ & $-5(1)$ \\
\hline$C(1)$ & $14(1)$ & $14(1)$ & $16(1)$ & $-8(1)$ & $-1(1)$ & $-2(1)$ \\
\hline$C(2)$ & 21(1) & $16(1)$ & $12(1)$ & $-6(1)$ & $-1(1)$ & $-7(1)$ \\
\hline$C(3)$ & $23(1)$ & $23(1)$ & $17(1)$ & $-3(1)$ & $-6(1)$ & $-8(1)$ \\
\hline$C(4)$ & $28(1)$ & $32(1)$ & $19(1)$ & $0(1)$ & $-6(1)$ & $-20(1)$ \\
\hline$C(5)$ & $40(1)$ & $22(1)$ & $25(1)$ & $-3(1)$ & $-4(1)$ & $-19(1)$ \\
\hline$C(6)$ & $34(1)$ & $14(1)$ & $34(1)$ & $-5(1)$ & $-4(1)$ & $-6(1)$ \\
\hline$C(7)$ & $21(1)$ & $16(1)$ & $28(1)$ & $-6(1)$ & $-3(1)$ & $-5(1)$ \\
\hline$C(8)$ & $17(1)$ & $13(1)$ & $15(1)$ & $-7(1)$ & $-2(1)$ & $-3(1)$ \\
\hline$C(9)$ & $27(1)$ & $18(1)$ & $16(1)$ & $-4(1)$ & $-6(1)$ & $-5(1)$ \\
\hline$C(10)$ & $32(1)$ & $17(1)$ & $17(1)$ & $-2(1)$ & $-3(1)$ & $-6(1)$ \\
\hline$C(11)$ & $23(1)$ & $18(1)$ & $20(1)$ & $-5(1)$ & $0(1)$ & $-8(1)$ \\
\hline$C(12)$ & $17(1)$ & $17(1)$ & $19(1)$ & $-7(1)$ & $-2(1)$ & $-5(1)$ \\
\hline$C(13)$ & $22(1)$ & $16(1)$ & $13(1)$ & $-4(1)$ & $-6(1)$ & $-4(1)$ \\
\hline$C(14)$ & $19(1)$ & $17(1)$ & $11(1)$ & $-5(1)$ & $-2(1)$ & $-4(1)$ \\
\hline$C(15)$ & 16(1) & $19(1)$ & $13(1)$ & $-6(1)$ & $-1(1)$ & $-4(1)$ \\
\hline$C(16)$ & 21(1) & $19(1)$ & $12(1)$ & $-4(1)$ & $-2(1)$ & $-6(1)$ \\
\hline$C(17)$ & $23(1)$ & $17(1)$ & $14(1)$ & $-3(1)$ & $-5(1)$ & $-5(1)$ \\
\hline$C(18)$ & $22(1)$ & $24(1)$ & $26(1)$ & $-2(1)$ & $-12(1)$ & $-4(1)$ \\
\hline$C(19)$ & $25(1)$ & $15(1)$ & $20(1)$ & $-5(1)$ & $-5(1)$ & $-5(1)$ \\
\hline$C(20)$ & $17(1)$ & $25(1)$ & $26(1)$ & $-7(1)$ & $-5(1)$ & $1(1)$ \\
\hline$C(21)$ & $24(1)$ & $26(1)$ & $19(1)$ & $-5(1)$ & $0(1)$ & $-12(1)$ \\
\hline$C(22)$ & $35(1)$ & $17(1)$ & $24(1)$ & $-1(1)$ & $-10(1)$ & $-3(1)$ \\
\hline $\mathrm{Rh}(2)$ & $12(1)$ & $12(1)$ & 18(1) & $-4(1)$ & $-3(1)$ & $-3(1)$ \\
\hline $\mathrm{Cl}(2)$ & $17(1)$ & $21(1)$ & $32(1)$ & $-8(1)$ & $-10(1)$ & $-3(1)$ \\
\hline $\mathrm{N}(3)$ & $14(1)$ & 18(1) & $21(1)$ & $-7(1)$ & $-3(1)$ & $0(1)$ \\
\hline $\mathrm{N}(4)$ & 16(1) & $15(1)$ & $23(1)$ & $-5(1)$ & $-2(1)$ & $-3(1)$ \\
\hline$C(23)$ & $12(1)$ & $14(1)$ & $21(1)$ & $-4(1)$ & $-4(1)$ & $-3(1)$ \\
\hline
\end{tabular}




\begin{tabular}{lllllll}
$C(24)$ & $17(1)$ & $16(1)$ & $21(1)$ & $-6(1)$ & $-1(1)$ & $-8(1)$ \\
$C(25)$ & $21(1)$ & $21(1)$ & $24(1)$ & $-4(1)$ & $-2(1)$ & $-8(1)$ \\
$C(26)$ & $33(1)$ & $29(1)$ & $23(1)$ & $-2(1)$ & $-3(1)$ & $-16(1)$ \\
$C(27)$ & $42(1)$ & $31(1)$ & $24(1)$ & $-11(1)$ & $9(1)$ & $-14(1)$ \\
$C(28)$ & $36(1)$ & $26(1)$ & $35(1)$ & $-12(1)$ & $7(1)$ & $0(1)$ \\
$C(29)$ & $27(1)$ & $20(1)$ & $26(1)$ & $-5(1)$ & $0(1)$ & $-3(1)$ \\
$C(30)$ & $13(1)$ & $15(1)$ & $26(1)$ & $-8(1)$ & $-4(1)$ & $-1(1)$ \\
$C(31)$ & $23(1)$ & $17(1)$ & $35(1)$ & $-10(1)$ & $-4(1)$ & $1(1)$ \\
$C(32)$ & $32(1)$ & $25(1)$ & $40(1)$ & $-21(1)$ & $-6(1)$ & $6(1)$ \\
$C(33)$ & $31(1)$ & $35(1)$ & $28(1)$ & $-17(1)$ & $-2(1)$ & $4(1)$ \\
$C(34)$ & $21(1)$ & $28(1)$ & $22(1)$ & $-8(1)$ & $-1(1)$ & $0(1)$ \\
$C(35)$ & $20(1)$ & $16(1)$ & $32(1)$ & $3(1)$ & $-9(1)$ & $-8(1)$ \\
$C(36)$ & $18(1)$ & $23(1)$ & $28(1)$ & $1(1)$ & $-4(1)$ & $-11(1)$ \\
$C(37)$ & $14(1)$ & $18(1)$ & $31(1)$ & $-2(1)$ & $-6(1)$ & $-7(1)$ \\
$C(38)$ & $21(1)$ & $15(1)$ & $29(1)$ & $-3(1)$ & $-11(1)$ & $-10(1)$ \\
$C(39)$ & $20(1)$ & $13(1)$ & $31(1)$ & $-5(1)$ & $-6(1)$ & $-7(1)$ \\
$C(40)$ & $27(1)$ & $23(1)$ & $45(1)$ & $9(1)$ & $-14(1)$ & $-6(1)$ \\
$C(41)$ & $27(1)$ & $47(1)$ & $29(1)$ & $2(1)$ & $2(1)$ & $-16(1)$ \\
$C(42)$ & $17(1)$ & $26(1)$ & $45(1)$ & $-3(1)$ & $-7(1)$ & $-2(1)$ \\
$C(43)$ & $38(1)$ & $26(1)$ & $30(1)$ & $-1(1)$ & $-18(1)$ & $-14(1)$ \\
$C(44)$ & $30(1)$ & $18(1)$ & $44(1)$ & $-13(1)$ & $-2(1)$ & $-5(1)$ \\
& & & & & & \\
\hline
\end{tabular}


Table 5. Hydrogen coordinates $\left(\times 10^{4}\right)$ and isotropic displacement parameters $\left(\AA^{2} \times 10^{3}\right)$ for 007c-20063.

\begin{tabular}{|c|c|c|c|c|}
\hline & $x$ & $y$ & z & $\mathrm{U}(\mathrm{eq})$ \\
\hline $\mathrm{H}(3)$ & -2133 & 3806 & 1840 & 24 \\
\hline $\mathrm{H}(4)$ & -2940 & 2304 & 2036 & 29 \\
\hline$H(5)$ & -1236 & 884 & 2235 & 33 \\
\hline $\mathrm{H}(6)$ & 1234 & 955 & 2348 & 33 \\
\hline $\mathrm{H}(7)$ & 1995 & 2463 & 2257 & 26 \\
\hline $\mathrm{H}(9)$ & 488 & 6074 & -112 & 24 \\
\hline$H(10)$ & 1802 & 7376 & -310 & 26 \\
\hline$H(11)$ & 3045 & 7635 & 753 & 24 \\
\hline$H(12)$ & 2914 & 6601 & 1991 & 20 \\
\hline $\mathrm{H}(18 \mathrm{~A})$ & 4455 & 4297 & 3732 & 34 \\
\hline $\mathrm{H}(18 \mathrm{~B})$ & 3828 & 4613 & 4684 & 34 \\
\hline $\mathrm{H}(18 \mathrm{C})$ & 4088 & 5419 & 3905 & 34 \\
\hline$H(19 A)$ & 2463 & 6731 & 3633 & 29 \\
\hline $\mathrm{H}(19 \mathrm{~B})$ & 851 & 7002 & 4249 & 29 \\
\hline $\mathrm{H}(19 \mathrm{C})$ & 972 & 7060 & 3238 & 29 \\
\hline $\mathrm{H}(20 \mathrm{~A})$ & -1353 & 6562 & 3099 & 34 \\
\hline $\mathrm{H}(20 \mathrm{~B})$ & -2153 & 6321 & 4067 & 34 \\
\hline $\mathrm{H}(20 \mathrm{C})$ & -2269 & 5680 & 3336 & 34 \\
\hline $\mathrm{H}(21 \mathrm{~A})$ & -1914 & 4026 & 3545 & 33 \\
\hline $\mathrm{H}(21 \mathrm{~B})$ & -1901 & 3806 & 4542 & 33 \\
\hline $\mathrm{H}(21 \mathrm{C})$ & -837 & 3090 & 3873 & 33 \\
\hline $\mathrm{H}(22 \mathrm{~A})$ & 1595 & 2536 & 3994 & 37 \\
\hline $\mathrm{H}(22 \mathrm{~B})$ & 2096 & 2844 & 4801 & 37 \\
\hline $\mathrm{H}(22 \mathrm{C})$ & 3209 & 2853 & 3887 & 37 \\
\hline $\mathrm{H}(25)$ & 5216 & 7024 & 4486 & 26 \\
\hline $\mathrm{H}(26)$ & 3937 & 7421 & 5852 & 33 \\
\hline $\mathrm{H}(27)$ & 2022 & 8772 & 6019 & 40 \\
\hline $\mathrm{H}(28)$ & 1431 & 9751 & 4825 & 41 \\
\hline $\mathrm{H}(29)$ & 2759 & 9394 & 3456 & 30 \\
\hline $\mathrm{H}(31)$ & 5855 & 5376 & 2075 & 30 \\
\hline
\end{tabular}




\begin{tabular}{|c|c|c|c|c|}
\hline $\mathrm{H}(32)$ & 6755 & 5188 & 618 & 39 \\
\hline $\mathrm{H}(33)$ & 7248 & 6540 & -322 & 39 \\
\hline $\mathrm{H}(34)$ & 6857 & 8049 & 216 & 29 \\
\hline $\mathrm{H}(40 \mathrm{~A})$ & 5448 & 11120 & 466 & 47 \\
\hline $\mathrm{H}(40 \mathrm{~B})$ & 5126 & 11825 & 1218 & 47 \\
\hline $\mathrm{H}(40 \mathrm{C})$ & 4024 & 11071 & 1234 & 47 \\
\hline $\mathrm{H}(41 \mathrm{~A})$ & 8671 & 9106 & 207 & 52 \\
\hline $\mathrm{H}(41 \mathrm{~B})$ & 8642 & 10250 & 115 & 52 \\
\hline $\mathrm{H}(41 \mathrm{C})$ & 7234 & 9832 & -41 & 52 \\
\hline$H(42 A)$ & 9277 & 8036 & 2375 & 45 \\
\hline $\mathrm{H}(42 \mathrm{~B})$ & 10156 & 8776 & 1717 & 45 \\
\hline $\mathrm{H}(42 \mathrm{C})$ & 9325 & 8050 & 1375 & 45 \\
\hline$H(43 A)$ & 6309 & 9360 & 4009 & 43 \\
\hline $\mathrm{H}(43 \mathrm{~B})$ & 8035 & 9498 & 3692 & 43 \\
\hline $\mathrm{H}(43 \mathrm{C})$ & 7557 & 8483 & 3624 & 43 \\
\hline $\mathrm{H}(44 \mathrm{~A})$ & 3617 & 10993 & 2913 & 46 \\
\hline $\mathrm{H}(44 \mathrm{~B})$ & 4910 & 11540 & 3047 & 46 \\
\hline $\mathrm{H}(44 \mathrm{C})$ & 4518 & 10612 & 3668 & 46 \\
\hline
\end{tabular}


Table 6. Torsion angles [ $\left.{ }^{\circ}\right]$ for 007c-20063.

\begin{tabular}{|c|c|}
\hline $\mathrm{C}(8)-\mathrm{N}(2)-\mathrm{C}(1)-\mathrm{C}(2)$ & $-173.89(15)$ \\
\hline$C(8)-N(2)-C(1)-R h(1)$ & $6.0(2)$ \\
\hline$N(2)-C(1)-C(2)-C(7)$ & $126.86(18)$ \\
\hline$R h(1)-C(1)-C(2)-C(7)$ & $-53.1(2)$ \\
\hline$N(2)-C(1)-C(2)-C(3)$ & $-51.4(2)$ \\
\hline$R h(1)-C(1)-C(2)-C(3)$ & $128.73(15)$ \\
\hline$C(7)-C(2)-C(3)-C(4)$ & $-0.1(3)$ \\
\hline$C(1)-C(2)-C(3)-C(4)$ & $178.16(16)$ \\
\hline$C(2)-C(3)-C(4)-C(5)$ & $-2.6(3)$ \\
\hline$C(3)-C(4)-C(5)-C(6)$ & $2.6(3)$ \\
\hline$C(4)-C(5)-C(6)-C(7)$ & $-0.1(3)$ \\
\hline$C(3)-C(2)-C(7)-C(6)$ & $2.6(3)$ \\
\hline$C(1)-C(2)-C(7)-C(6)$ & $-175.60(17)$ \\
\hline$C(5)-C(6)-C(7)-C(2)$ & $-2.6(3)$ \\
\hline $\mathrm{C}(12)-\mathrm{N}(1)-\mathrm{C}(8)-\mathrm{N}(2)$ & $174.92(15)$ \\
\hline $\mathrm{Rh}(1)-\mathrm{N}(1)-\mathrm{C}(8)-\mathrm{N}(2)$ & $-1.29(19)$ \\
\hline $\mathrm{C}(12)-\mathrm{N}(1)-\mathrm{C}(8)-\mathrm{C}(9)$ & $-2.0(2)$ \\
\hline $\mathrm{Rh}(1)-\mathrm{N}(1)-\mathrm{C}(8)-\mathrm{C}(9)$ & $-178.18(13)$ \\
\hline $\mathrm{C}(1)-\mathrm{N}(2)-\mathrm{C}(8)-\mathrm{N}(1)$ & $-2.9(2)$ \\
\hline $\mathrm{C}(1)-\mathrm{N}(2)-\mathrm{C}(8)-\mathrm{C}(9)$ & $174.00(16)$ \\
\hline$N(1)-C(8)-C(9)-C(10)$ & $1.8(3)$ \\
\hline$N(2)-C(8)-C(9)-C(10)$ & $-174.92(17)$ \\
\hline $\mathrm{C}(8)-\mathrm{C}(9)-\mathrm{C}(10)-\mathrm{C}(11)$ & $-0.5(3)$ \\
\hline$C(9)-C(10)-C(11)-C(12)$ & $-0.6(3)$ \\
\hline $\mathrm{C}(8)-\mathrm{N}(1)-\mathrm{C}(12)-\mathrm{C}(11)$ & $0.8(3)$ \\
\hline $\operatorname{Rh}(1)-\mathrm{N}(1)-\mathrm{C}(12)-\mathrm{C}(11)$ & $176.44(13)$ \\
\hline $\mathrm{C}(10)-\mathrm{C}(11)-\mathrm{C}(12)-\mathrm{N}(1)$ & $0.5(3)$ \\
\hline$C(17)-C(13)-C(14)-C(15)$ & $-0.98(19)$ \\
\hline$C(18)-C(13)-C(14)-C(15)$ & $176.45(17)$ \\
\hline $\mathrm{Rh}(1)-\mathrm{C}(13)-\mathrm{C}(14)-\mathrm{C}(15)$ & $56.64(11)$ \\
\hline$C(17)-C(13)-C(14)-C(19)$ & $178.83(16)$ \\
\hline$C(18)-C(13)-C(14)-C(19)$ & $-3.7(3)$ \\
\hline $\mathrm{Rh}(1)-\mathrm{C}(13)-\mathrm{C}(14)-\mathrm{C}(19)$ & $-123.55(18)$ \\
\hline$C(17)-C(13)-C(14)-R h(1)$ & $-57.62(11)$ \\
\hline
\end{tabular}




\begin{tabular}{|c|c|}
\hline$C(18)-C(13)-C(14)-R h(1)$ & 119.81(18) \\
\hline$C(13)-C(14)-C(15)-C(16)$ & $5.10(19)$ \\
\hline$C(19)-C(14)-C(15)-C(16)$ & $-174.72(16)$ \\
\hline $\mathrm{Rh}(1)-\mathrm{C}(14)-\mathrm{C}(15)-\mathrm{C}(16)$ & $65.62(11)$ \\
\hline$C(13)-C(14)-C(15)-C(20)$ & $176.14(16)$ \\
\hline$C(19)-C(14)-C(15)-C(20)$ & $-3.7(3)$ \\
\hline $\mathrm{Rh}(1)-\mathrm{C}(14)-\mathrm{C}(15)-\mathrm{C}(20)$ & $-123.34(18)$ \\
\hline$C(13)-C(14)-C(15)-R h(1)$ & $-60.52(12)$ \\
\hline$C(19)-C(14)-C(15)-R h(1)$ & 119.66(16) \\
\hline$C(14)-C(15)-C(16)-C(17)$ & $-7.27(19)$ \\
\hline$C(20)-C(15)-C(16)-C(17)$ & $-178.26(16)$ \\
\hline $\mathrm{Rh}(1)-\mathrm{C}(15)-\mathrm{C}(16)-\mathrm{C}(17)$ & $60.28(12)$ \\
\hline$C(14)-C(15)-C(16)-C(21)$ & $170.04(16)$ \\
\hline$C(20)-C(15)-C(16)-C(21)$ & $-0.9(3)$ \\
\hline $\mathrm{Rh}(1)-\mathrm{C}(15)-\mathrm{C}(16)-\mathrm{C}(21)$ & $-122.41(17)$ \\
\hline$C(14)-C(15)-C(16)-R h(1)$ & $-67.54(11)$ \\
\hline$C(20)-C(15)-C(16)-R h(1)$ & $121.47(18)$ \\
\hline$C(15)-C(16)-C(17)-C(13)$ & $6.69(19)$ \\
\hline$C(21)-C(16)-C(17)-C(13)$ & $-170.55(16)$ \\
\hline $\mathrm{Rh}(1)-\mathrm{C}(16)-\mathrm{C}(17)-\mathrm{C}(13)$ & $65.97(12)$ \\
\hline$C(15)-C(16)-C(17)-C(22)$ & $-179.16(17)$ \\
\hline$C(21)-C(16)-C(17)-C(22)$ & $3.6(3)$ \\
\hline $\mathrm{Rh}(1)-\mathrm{C}(16)-\mathrm{C}(17)-\mathrm{C}(22)$ & $-119.88(18)$ \\
\hline$C(15)-C(16)-C(17)-R h(1)$ & $-59.28(12)$ \\
\hline$C(21)-C(16)-C(17)-\operatorname{Rh}(1)$ & $123.48(18)$ \\
\hline$C(14)-C(13)-C(17)-C(16)$ & $-3.55(19)$ \\
\hline$C(18)-C(13)-C(17)-C(16)$ & $178.95(16)$ \\
\hline $\mathrm{Rh}(1)-\mathrm{C}(13)-\mathrm{C}(17)-\mathrm{C}(16)$ & $-64.03(12)$ \\
\hline$C(14)-C(13)-C(17)-C(22)$ & $-177.84(17)$ \\
\hline$C(18)-C(13)-C(17)-C(22)$ & $4.7(3)$ \\
\hline $\operatorname{Rh}(1)-C(13)-C(17)-C(22)$ & $121.68(18)$ \\
\hline$C(14)-C(13)-C(17)-\operatorname{Rh}(1)$ & $60.48(12)$ \\
\hline$C(18)-C(13)-C(17)-\operatorname{Rh}(1)$ & $-117.02(17)$ \\
\hline $\mathrm{C}(30)-\mathrm{N}(4)-\mathrm{C}(23)-\mathrm{C}(24)$ & $174.90(15)$ \\
\hline $\mathrm{C}(30)-\mathrm{N}(4)-\mathrm{C}(23)-\mathrm{Rh}(2)$ & $-4.2(2)$ \\
\hline$N(4)-C(23)-C(24)-C(29)$ & $-137.46(18)$ \\
\hline
\end{tabular}




\begin{tabular}{|c|c|}
\hline $\mathrm{Rh}(2)-\mathrm{C}(23)-\mathrm{C}(24)-\mathrm{C}(29)$ & $41.6(2)$ \\
\hline$N(4)-C(23)-C(24)-C(25)$ & $41.1(2)$ \\
\hline $\mathrm{Rh}(2)-\mathrm{C}(23)-\mathrm{C}(24)-\mathrm{C}(25)$ & $-139.84(15)$ \\
\hline$C(29)-C(24)-C(25)-C(26)$ & $-0.8(3)$ \\
\hline$C(23)-C(24)-C(25)-C(26)$ & $-179.35(16)$ \\
\hline$C(24)-C(25)-C(26)-C(27)$ & $1.5(3)$ \\
\hline$C(25)-C(26)-C(27)-C(28)$ & $-0.9(3)$ \\
\hline$C(26)-C(27)-C(28)-C(29)$ & $-0.3(3)$ \\
\hline$C(27)-C(28)-C(29)-C(24)$ & $0.9(3)$ \\
\hline$C(25)-C(24)-C(29)-C(28)$ & $-0.4(3)$ \\
\hline$C(23)-C(24)-C(29)-C(28)$ & $178.17(18)$ \\
\hline $\mathrm{C}(34)-\mathrm{N}(3)-\mathrm{C}(30)-\mathrm{N}(4)$ & $-174.89(16)$ \\
\hline $\mathrm{Rh}(2)-\mathrm{N}(3)-\mathrm{C}(30)-\mathrm{N}(4)$ & $2.07(19)$ \\
\hline $\mathrm{C}(34)-\mathrm{N}(3)-\mathrm{C}(30)-\mathrm{C}(31)$ & $1.4(3)$ \\
\hline $\operatorname{Rh}(2)-N(3)-C(30)-C(31)$ & $178.35(14)$ \\
\hline $\mathrm{C}(23)-\mathrm{N}(4)-\mathrm{C}(30)-\mathrm{N}(3)$ & $1.2(2)$ \\
\hline$C(23)-N(4)-C(30)-C(31)$ & $-175.08(17)$ \\
\hline$N(3)-C(30)-C(31)-C(32)$ & $-1.2(3)$ \\
\hline$N(4)-C(30)-C(31)-C(32)$ & $174.89(18)$ \\
\hline$C(30)-C(31)-C(32)-C(33)$ & $0.3(3)$ \\
\hline$C(31)-C(32)-C(33)-C(34)$ & $0.5(3)$ \\
\hline $\mathrm{C}(30)-\mathrm{N}(3)-\mathrm{C}(34)-\mathrm{C}(33)$ & $-0.6(3)$ \\
\hline $\operatorname{Rh}(2)-N(3)-C(34)-C(33)$ & $-177.07(15)$ \\
\hline $\mathrm{C}(32)-\mathrm{C}(33)-\mathrm{C}(34)-\mathrm{N}(3)$ & $-0.3(3)$ \\
\hline$C(39)-C(35)-C(36)-C(37)$ & $0.7(2)$ \\
\hline $\mathrm{C}(40)-\mathrm{C}(35)-\mathrm{C}(36)-\mathrm{C}(37)$ & $-178.74(17)$ \\
\hline $\operatorname{Rh}(2)-C(35)-C(36)-C(37)$ & $-56.85(12)$ \\
\hline$C(39)-C(35)-C(36)-C(41)$ & $-177.68(18)$ \\
\hline$C(40)-C(35)-C(36)-C(41)$ & $2.9(3)$ \\
\hline $\operatorname{Rh}(2)-C(35)-C(36)-C(41)$ & $124.8(2)$ \\
\hline $\mathrm{C}(39)-\mathrm{C}(35)-\mathrm{C}(36)-\mathrm{Rh}(2)$ & $57.55(12)$ \\
\hline$C(40)-C(35)-C(36)-R h(2)$ & $-121.89(19)$ \\
\hline $\mathrm{C}(35)-\mathrm{C}(36)-\mathrm{C}(37)-\mathrm{C}(38)$ & $-3.7(2)$ \\
\hline $\mathrm{C}(41)-\mathrm{C}(36)-\mathrm{C}(37)-\mathrm{C}(38)$ & $174.70(18)$ \\
\hline $\mathrm{Rh}(2)-\mathrm{C}(36)-\mathrm{C}(37)-\mathrm{C}(38)$ & $-64.40(11)$ \\
\hline$C(35)-C(36)-C(37)-C(42)$ & $-175.41(17)$ \\
\hline
\end{tabular}




\begin{tabular}{|c|c|}
\hline$C(41)-C(36)-C(37)-C(42)$ & $3.0(3)$ \\
\hline $\mathrm{Rh}(2)-\mathrm{C}(36)-\mathrm{C}(37)-\mathrm{C}(42)$ & $123.87(18)$ \\
\hline$C(35)-C(36)-C(37)-R h(2)$ & $60.72(13)$ \\
\hline$C(41)-C(36)-C(37)-R h(2)$ & $-120.90(19)$ \\
\hline $\mathrm{C}(36)-\mathrm{C}(37)-\mathrm{C}(38)-\mathrm{C}(39)$ & $5.23(19)$ \\
\hline$C(42)-C(37)-C(38)-C(39)$ & $176.84(17)$ \\
\hline $\mathrm{Rh}(2)-\mathrm{C}(37)-\mathrm{C}(38)-\mathrm{C}(39)$ & $-61.99(12)$ \\
\hline$C(36)-C(37)-C(38)-C(43)$ & $-171.97(17)$ \\
\hline$C(42)-C(37)-C(38)-C(43)$ & $-0.4(3)$ \\
\hline $\mathrm{Rh}(2)-\mathrm{C}(37)-\mathrm{C}(38)-\mathrm{C}(43)$ & $120.81(17)$ \\
\hline $\mathrm{C}(36)-\mathrm{C}(37)-\mathrm{C}(38)-\mathrm{Rh}(2)$ & $67.22(12)$ \\
\hline $\mathrm{C}(42)-\mathrm{C}(37)-\mathrm{C}(38)-\mathrm{Rh}(2)$ & $-121.17(18)$ \\
\hline $\mathrm{C}(37)-\mathrm{C}(38)-\mathrm{C}(39)-\mathrm{C}(35)$ & $-4.76(19)$ \\
\hline$C(43)-C(38)-C(39)-C(35)$ & $172.39(17)$ \\
\hline $\operatorname{Rh}(2)-C(38)-C(39)-C(35)$ & $-65.60(12)$ \\
\hline $\mathrm{C}(37)-\mathrm{C}(38)-\mathrm{C}(39)-\mathrm{C}(44)$ & $-173.04(17)$ \\
\hline $\mathrm{C}(43)-\mathrm{C}(38)-\mathrm{C}(39)-\mathrm{C}(44)$ & $4.1(3)$ \\
\hline $\operatorname{Rh}(2)-C(38)-C(39)-C(44)$ & $126.12(19)$ \\
\hline $\mathrm{C}(37)-\mathrm{C}(38)-\mathrm{C}(39)-\mathrm{Rh}(2)$ & $60.84(11)$ \\
\hline $\mathrm{C}(43)-\mathrm{C}(38)-\mathrm{C}(39)-\mathrm{Rh}(2)$ & $-122.02(18)$ \\
\hline $\mathrm{C}(36)-\mathrm{C}(35)-\mathrm{C}(39)-\mathrm{C}(38)$ & $2.5(2)$ \\
\hline$C(40)-C(35)-C(39)-C(38)$ & $-178.01(17)$ \\
\hline $\operatorname{Rh}(2)-C(35)-C(39)-C(38)$ & $62.75(11)$ \\
\hline$C(36)-C(35)-C(39)-C(44)$ & $171.37(17)$ \\
\hline$C(40)-C(35)-C(39)-C(44)$ & $-9.2(3)$ \\
\hline $\mathrm{Rh}(2)-\mathrm{C}(35)-\mathrm{C}(39)-\mathrm{C}(44)$ & $-128.42(18)$ \\
\hline $\mathrm{C}(36)-\mathrm{C}(35)-\mathrm{C}(39)-\mathrm{Rh}(2)$ & $-60.21(13)$ \\
\hline$C(40)-C(35)-C(39)-\operatorname{Rh}(2)$ & $119.24(18)$ \\
\hline
\end{tabular}

Symmetry transformations used to generate equivalent atoms: 